\title{
Carbon Atom Insertion into Pyrroles and Indoles Promoted by Chlorodiazirines Experimental Details and Spectra
}

\author{
Balu D. Dherange ${ }^{1}$, Patrick Q. Kelly ${ }^{1}$, Jordan P. Liles ${ }^{2}$, Matthew S. Sigman ${ }^{2}$, and Mark D. Levin ${ }^{1, *}$ \\ ${ }^{1}$ Department of Chemistry, University of Chicago, Chicago, IL 60637, United States \\ 2 Department of Chemistry, University of Utah, Salt Lake City, Utah 84112, United States
}

\section{Table of Contents}

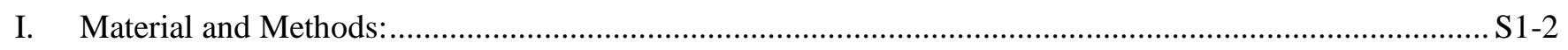

II. General Procedure for synthesis of 3 -substituted quinolines (3) / pyridines: ....................................... S1-3

III. Synthesis of Starting Materials

IIIA. General procedure for synthesis of chlorodiazirine $1: \ldots \ldots \ldots \ldots \ldots \ldots \ldots \ldots \ldots \ldots \ldots \ldots \ldots . . .51-21$

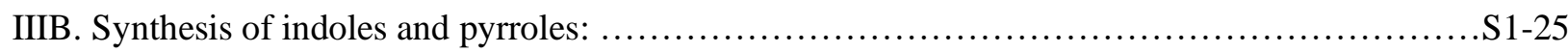

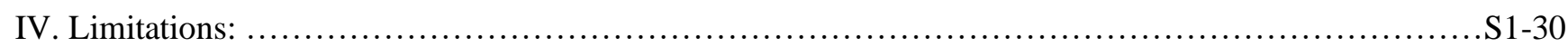

V. Comparison with Ciamician-Denstedt Reaction: .......................................... $11-31$

VI. Mechanistic Experiments

VIA. Effect of addition of tetrabutyl ammonium chloride: ............................. $1-33$

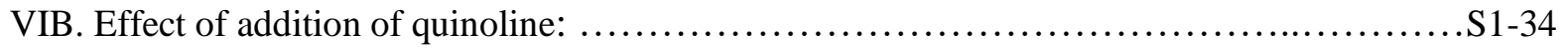

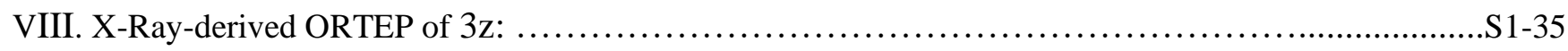

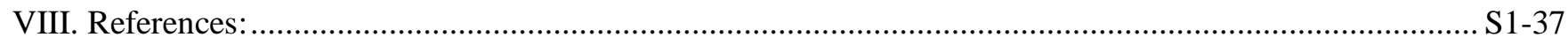

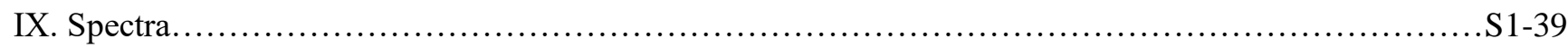




\section{Material and Methods}

Unless noted otherwise, all reactions were performed in oven-dried or flame-dried glassware under an atmosphere of dry $\mathrm{N}_{2} . \mathrm{CH}_{3} \mathrm{CN}$, THF, $\mathrm{Et}_{2} \mathrm{O}, \mathrm{CH}_{2} \mathrm{Cl}_{2}$, toluene, and $\mathrm{Et}_{3} \mathrm{~N}$ were dried by passing these previously degassed solvents through a PPT Solvent Purification System, and all other solvents were dried over molecular sieves (4A) and degassed prior to use or purchased an- hydrous and sealed under $\mathrm{N}_{2}$ (e.g. VWR Dri-solv or equivalent). Reaction temperatures were reported as the temperatures of the bath surrounding the flasks or vials. Sensitive reagents and solvents were transferred under nitrogen into a nitrogen-filled glovebox with standard techniques. Unless otherwise noted, all reagents were used as received. Analytical thin-layer chromatography (TLC) was carried out using $0.2 \mathrm{~mm}$ commercial silica gel plates (silica gel 60, F254) and visualized by UV irradiation or staining as indicated.

High resolution mass spectra were recorded on either an Agilent 6224 TOF High Resolution Accurate MS with electrospray ionization or an Agilent 7200B QTOF High Resolution Accurate Mass GCMS using an Agilent HP-5MS column with a temperature gradient of $50{ }^{\circ} \mathrm{C}$ to $200{ }^{\circ} \mathrm{C}$ over 15 minutes and electron impact ionization. All mass spectra were processed with an Agilent MassHunter Operating System. X-Ray crystallographic analysis data were collected using a Bruker D8 VENTURE with PHOTON 100 CMOS detector system. Nuclear magnetic resonance spectra $\left({ }^{1} \mathrm{H}-\mathrm{NMR},{ }^{13} \mathrm{C}-\mathrm{NMR}\right.$ and $\left.{ }^{19} \mathrm{~F}-\mathrm{NMR}\right)$ were recorded with Bruker spectrometers operating at 400 or $500 \mathrm{MHz}$ for $1 \mathrm{H}$. Chemical shifts are reported in parts per million (ppm, $\delta$ ), downfield from tetramethylsilane (TMS, $\delta=0.00 \mathrm{ppm}$ ) and are referenced to residual solvent $(\mathrm{CDCl} 3, \delta=7.26 \mathrm{ppm}(1 \mathrm{H})$ and $77.160 \mathrm{ppm}(13 \mathrm{C}))$. Coupling constants were reported in Hertz $(\mathrm{Hz})$. Data for 1H-NMR spectra were reported as follows: chem- ical shift (ppm, $\mathrm{s}=\operatorname{singlet}, \mathrm{d}=$ doublet, $\mathrm{t}=$ triplet, $\mathrm{q}=$ quartet, quin = quintet, $\mathrm{dd}=$ doublet of doublets, $\mathrm{td}=$ triplet of doublets, $\mathrm{ddd}=$ doublet of doublet of doublets, $\mathrm{m}=$ multiplet, coupling constant $(\mathrm{Hz})$, and integration).

CAUTION! Diazirines are reactive compounds that release gaseous byproducts. Though they are typically less reactive than the isomeric diazos and we have not encountered any stability issues with arylchlorocarbenes to date, lower molecular weight diazirines (e.g. methylchlorodiazirine) have been reported to detonate. Operations should be conducted behind a blast shield and scales should be limited whenever possible. 


\section{General Procedure for synthesis of 3-substituted quinolines (3) and pyridines (5) from indoles (2) and pyrroles (4)}

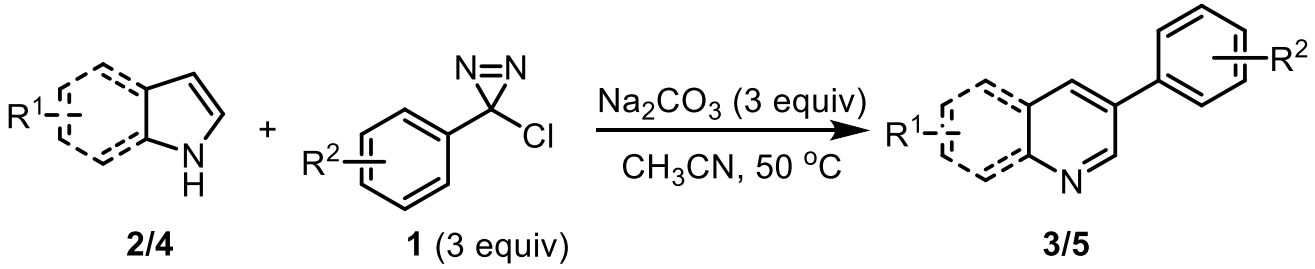

Oven dried 1-dram screw cap vial equipped with a stir bar and PTFE/white silicone septum was added indole 2 /pyrrole $4\left(0.2 \mathrm{mmol}\right.$.) and dry $\mathrm{CH}_{3} \mathrm{CN}(1 \mathrm{~mL})$, followed by diazirine $1(0.6 \mathrm{mmol})$ and $\mathrm{Na}_{2} \mathrm{CO}_{3}$ $(0.6 \mathrm{mmol})$. The vial then sealed with electric tape. The mixture was then allowed to stir at $50{ }^{\circ} \mathrm{C}$ for $12 \mathrm{~h}$ and then allowed to cool to $25^{\circ} \mathrm{C}$. The reaction mixture was quenched by the addition of saturated aqueous $\mathrm{NH}_{4} \mathrm{Cl}$ solution $(1 \mathrm{~mL})$, and the two phases were separated. The aqueous layer was extracted with EtOAc $(3 \times 3 \mathrm{~mL})$, and the combined organic layers were dried over anhydrous $\mathrm{Na}_{2} \mathrm{SO}_{4}$, filtered, and concentrated under reduced pressure. The obtained residue was purified by flash column chromatography to afford 3substituted quinoline 3/pyridine $\mathbf{5}$.

2-methyl-3-phenylquinoline (3a):

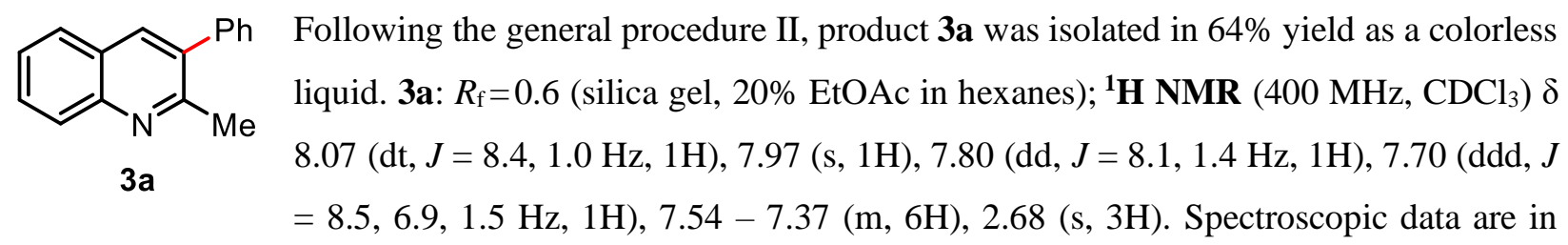
agreement with those in the literature. ${ }^{1}$

\section{3-(4-fluorophenyl)-2-methylquinoline (3b):}

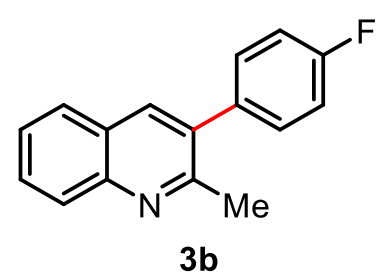

data are in agreement with those in the literature. ${ }^{2}$ 


\section{2-methyl-3-(p-tolyl)quinoline (3c):}

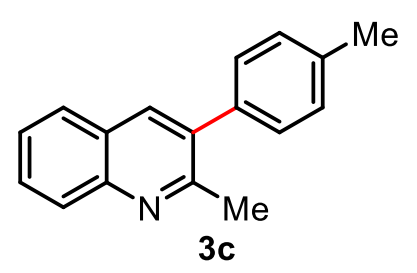

Following the general procedure II, product $3 \mathbf{c}$ was isolated in $62 \%$ yield as a yellow viscous liquid. $3 \mathrm{c}$ : $R_{\mathrm{f}}=0.3$ (silica gel, $10 \%$ EtOAc in hexanes); ${ }^{1} \mathbf{H}$ NMR $\left(400 \mathrm{MHz}, \mathrm{CDCl}_{3}\right) \delta 8.09-8.03(\mathrm{~m}, 1 \mathrm{H}), 7.94(\mathrm{~s}, 1 \mathrm{H}), 7.79(\mathrm{dd}, J=8.0,1.4$ $\mathrm{Hz}, 1 \mathrm{H}), 7.69$ (ddd, $J=8.4,6.9,1.5 \mathrm{~Hz}, 1 \mathrm{H}), 7.50(\mathrm{ddd}, J=8.1,6.9,1.2 \mathrm{~Hz}$, 1H), $7.33-7.27(\mathrm{~m}, 4 \mathrm{H}), 2.68(\mathrm{~s}, 3 \mathrm{H}), 2.44(\mathrm{~s}, 3 \mathrm{H}) ;{ }^{13} \mathbf{C}\left\{{ }^{1} \mathbf{H}\right\}$ NMR $(101 \mathrm{MHz}$, $\left.\mathrm{CDCl}_{3}\right) \delta 157.7,147.1,137.5,137.1,136.2,135.9,129.4,129.3,129.2,128.5,127.6,127.1,126.1,24.8$, 21.4; HRMS (ESI-TOF) calcd for $\mathrm{C}_{17} \mathrm{H}_{16} \mathrm{~N}^{+}[\mathrm{M}+\mathrm{H}]^{+} 234.1277$, found 234.1284

\section{2-methyl-3-(4-nitrophenyl)quinoline (3d):}

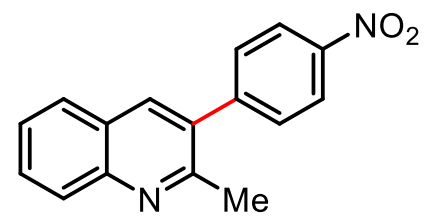

$3 d$

Following the general procedure II, product 3d was isolated in $59 \%$ yield as a yellow liquid along with $9 \%$ recovered starting material. $3 \mathrm{~d}: R_{\mathrm{f}}=0.3$ (silica gel, 20\% EtOAc in hexanes); ${ }^{1} \mathbf{H}$ NMR $\left(400 \mathrm{MHz}, \mathrm{CDCl}_{3}\right) \delta 8.36(\mathrm{~d}, J=8.7$ $\mathrm{Hz}, 2 \mathrm{H}), 8.08(\mathrm{dd}, J=8.6,1.1 \mathrm{~Hz}, 1 \mathrm{H}), 7.99(\mathrm{~s}, 1 \mathrm{H}), 7.83(\mathrm{dd}, J=8.1,1.4 \mathrm{~Hz}$,

$1 \mathrm{H}), 7.75$ (ddd, $J=8.5,6.9,1.5 \mathrm{~Hz}, 1 \mathrm{H}), 7.61(\mathrm{~d}, J=8.7 \mathrm{~Hz}, 2 \mathrm{H}), 7.55$ (ddd, $J=8.1,6.8,1.2 \mathrm{~Hz}, 1 \mathrm{H}), 2.67(\mathrm{~s}, 3 \mathrm{H}) ;{ }^{13} \mathbf{C}\left\{{ }^{1} \mathbf{H}\right\} \mathbf{N M R}\left(101 \mathrm{MHz}, \mathrm{CDCl}_{3}\right) \delta 156.2,147.7,147.5,146.9$, 136.5, 133.6, 130.4, 130.3, 128.7, 127.7, 126.7, 126.6, 123.9, 24.7; HRMS (ESI-TOF) calcd for $\mathrm{C}_{16} \mathrm{H}_{13} \mathrm{~N}_{2} \mathrm{O}_{2}+[\mathrm{M}+\mathrm{H}]^{+}$265.0972, found 265.0975.

\section{3-(4-bromophenyl)-2-methylquinoline (3e):}

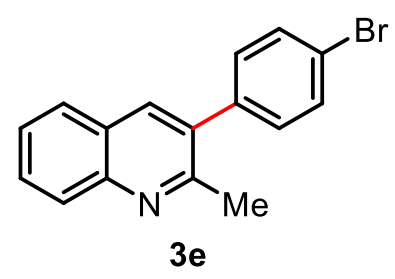

Following the general procedure II, product $\mathbf{3 e}$ was isolated in $67 \%$ yield as a yellow solid. 3e: $R_{\mathrm{f}}=0.5$ (silica gel, 20\% EtOAc in hexanes); ${ }^{1} \mathbf{H}$ NMR (400 $\left.\mathrm{MHz}, \mathrm{CDCl}_{3}\right) \delta 8.09-8.02(\mathrm{~m}, 1 \mathrm{H}), 7.94(\mathrm{~s}, 1 \mathrm{H}), 7.79(\mathrm{dd}, J=8.1,1.4 \mathrm{~Hz}, 1 \mathrm{H})$, 7.71 (ddd, $J=8.5,6.9,1.5 \mathrm{~Hz}, 1 \mathrm{H}), 7.65-7.58(\mathrm{~m}, 2 \mathrm{H}), 7.52$ (ddd, $J=8.1,6.9$, $1.2 \mathrm{~Hz}, 1 \mathrm{H}), 7.31-7.27(\mathrm{~m}, 2 \mathrm{H}), 2.65(\mathrm{~s}, 3 \mathrm{H}) ;{ }^{13} \mathbf{C}\left\{{ }^{1} \mathbf{H}\right\}$ NMR $(101 \mathrm{MHz}$, $\left.\mathrm{CDCl}_{3}\right) \delta 157.1,147.3,139.0,136.2,134.6,131.8,131.0,129.8,128.6,127.6,126.9,126.4,122.1,24.7$; HRMS (ESI-TOF) calcd for $\mathrm{C}_{16} \mathrm{H}_{13} \mathrm{BrN}^{+}[\mathrm{M}+\mathrm{H}]^{+}$298.0226, found 298.0235.

\section{2-methyl-3-(pyridin-2-yl)quinoline (3f):}

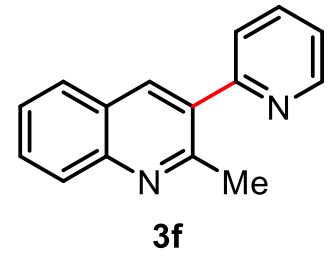

Following the general procedure II,* product $\mathbf{3 f}$ was isolated in $53 \%$ yield as a yellow solid along with $16 \%$ recovered starting material. 3f: $R_{\mathrm{f}}=0.3$ (silica gel, $20 \%$ EtOAc in hexanes); ${ }^{1} \mathbf{H}$ NMR $\left(400 \mathrm{MHz}, \mathrm{CDCl}_{3}\right) \delta 8.77$ (ddd, $J=4.8,1.9,1.0 \mathrm{~Hz}$, 1H), 8.17 (s, 1H), 8.07 (dd, $J=8.5,1.2 \mathrm{~Hz}, 1 \mathrm{H}), 7.86-7.80$ (m, 2H), 7.72 (ddd, $J$ $=8.4,6.9,1.4 \mathrm{~Hz}, 1 \mathrm{H}), 7.53(\mathrm{dt}, J=7.7,1.1 \mathrm{~Hz}, 2 \mathrm{H}), 7.34(\mathrm{ddd}, J=7.6,4.9,1.1 \mathrm{~Hz}$, 
1H), 2.78 (s, 3H); ${ }^{13} \mathbf{C}\left\{{ }^{1} \mathbf{H}\right\}$ NMR (101 MHz, $\left.\mathrm{CDCl}_{3}\right) \delta 158.4,157.3,149.7,147.5,136.9,136.7,134.5$, 130.0, 128.6, 127.9, 126.8, 126.3, 124.4, 122.5, 24.7; HRMS (ESI-TOF) calcd for $\mathrm{C}_{15} \mathrm{H}_{13} \mathrm{~N}_{2}{ }^{+}[\mathrm{M}+\mathrm{H}]^{+}$ 221.1073 , found 221.1081 .

* 5 equivalents of corresponding halo diazirine was used.

\section{2-ethyl-3-(m-tolyl)quinoline (3g):}

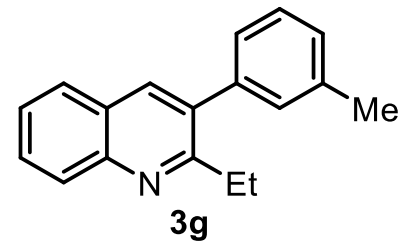

Following the general procedure II, product $\mathbf{3 g}$ was isolated in $70 \%$ yield as a colorless liquid. 3g: $R_{\mathrm{f}}=0.6$ (silica gel, $20 \%$ EtOAc in hexanes); ${ }^{1} \mathbf{H}$ NMR (400 $\left.\mathrm{MHz}, \mathrm{CDCl}_{3}\right) \delta 8.10(\mathrm{~d}, J=8.5 \mathrm{~Hz}, 1 \mathrm{H}), 7.93(\mathrm{~s}, 1 \mathrm{H}), 7.78(\mathrm{dd}, J=8.1,1.5 \mathrm{~Hz}$, $1 \mathrm{H}), 7.69(\mathrm{ddd}, J=8.5,6.9,1.5 \mathrm{~Hz}, 1 \mathrm{H}), 7.50(\mathrm{ddd}, J=8.1,6.8,1.2 \mathrm{~Hz}, 1 \mathrm{H})$, $7.36(\mathrm{t}, J=7.5 \mathrm{~Hz}, 1 \mathrm{H}), 7.26-7.15(\mathrm{~m}, 3 \mathrm{H}), 2.97(\mathrm{q}, J=7.5 \mathrm{~Hz}, 2 \mathrm{H}), 2.44(\mathrm{~s}, 3 \mathrm{H}), 1.24(\mathrm{t}, J=7.5 \mathrm{~Hz}, 3 \mathrm{H})$; ${ }^{13} \mathbf{C}\left\{{ }^{1} \mathbf{H}\right\}$ NMR $\left(101 \mathrm{MHz}, \mathrm{CDCl}_{3}\right) \delta 162.3,147.4,140.1,138.2,136.5,135.7,130.1,129.3,128.8,128.4$, 128.3, 127.6, 126.8, 126.5, 126.1, 29.9, 21.6, 13.9; HRMS (ESI-TOF) calcd for $\mathrm{C}_{18} \mathrm{H}_{18} \mathrm{~N}^{+}[\mathrm{M}+\mathrm{H}]^{+}$ 248.1434 , found 248.1437 .

\section{3-(4-chlorophenyl)-2-ethylquinoline (3h):}

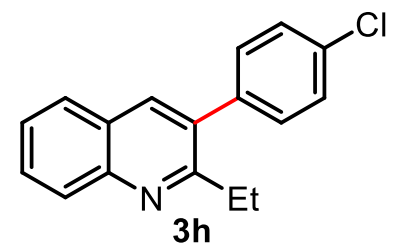

Following the general procedure II, product $\mathbf{3 h}$ was isolated in $83 \%$ yield as a yellow solid. 3h: $R_{\mathrm{f}}=0.5$ (silica gel, 20\% EtOAc in hexanes); ${ }^{1} \mathbf{H}$ NMR (400 $\mathrm{MHz}, \mathrm{CDCl}_{3} \delta 8.10(\mathrm{dd}, J=8.5,1.1 \mathrm{~Hz}, 1 \mathrm{H}), 7.95-7.87(\mathrm{~m}, 1 \mathrm{H}), 7.79(\mathrm{dd}, J=$ $8.1,1.4 \mathrm{~Hz}, 1 \mathrm{H}), 7.71(\mathrm{ddd}, J=8.4,6.9,1.5 \mathrm{~Hz}, 1 \mathrm{H}), 7.51(\mathrm{ddd}, J=8.1,6.9,1.2$ $\mathrm{Hz}, 1 \mathrm{H}), 7.48-7.42(\mathrm{~m}, 2 \mathrm{H}), 7.37-7.30(\mathrm{~m}, 2 \mathrm{H}), 2.95(\mathrm{q}, J=7.5 \mathrm{~Hz}, 2 \mathrm{H}), 1.23(\mathrm{t}, J=7.5 \mathrm{~Hz}, 3 \mathrm{H}) ;{ }^{13} \mathbf{C}\left\{{ }^{1} \mathbf{H}\right\}$ NMR (101 MHz, $\left.\mathrm{CDCl}_{3}\right) \delta 161.9,147.5,138.6,136.6,134.4,133.9,130.7,129.6,128.8,128.7,127.6$, 126.7, 126.3, 29.9, 13.9; HRMS (ESI-TOF) calcd for $\mathrm{C}_{17} \mathrm{H}_{15} \mathrm{ClN}^{+}[\mathrm{M}+\mathrm{H}]^{+}$268.0888, found 268.0894.

\section{2-ethyl-3-(3-nitrophenyl)quinoline (3i):}

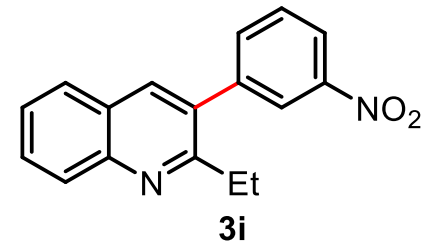

Following the general procedure II, product $3 \mathbf{i}$ was isolated in $83 \%$ yield as a yellow viscous liquid. 3i: $R_{\mathrm{f}}=0.4$ (silica gel, $20 \%$ EtOAc in hexanes); ${ }^{1} \mathbf{H}$ NMR $\left(400 \mathrm{MHz}, \mathrm{CDCl}_{3}\right) \delta 8.30(\mathrm{pd}, J=2.3,1.2 \mathrm{~Hz}, 2 \mathrm{H}), 8.15-8.08(\mathrm{~m}$, 1H), 7.98 (s, 1H), $7.81(\mathrm{dd}, J=8.1,1.4 \mathrm{~Hz}, 1 \mathrm{H}), 7.75$ (ddd, $J=8.6,6.8,1.4$ $\mathrm{Hz}, 2 \mathrm{H}), 7.67(\mathrm{dd}, J=8.8,7.6 \mathrm{~Hz}, 1 \mathrm{H}), 7.55(\mathrm{ddd}, J=8.1,6.8,1.2 \mathrm{~Hz}, 1 \mathrm{H}), 2.94(\mathrm{q}, J=7.5 \mathrm{~Hz}, 2 \mathrm{H}), 1.26$ $(\mathrm{t}, J=7.5 \mathrm{~Hz}, 3 \mathrm{H}) ;{ }^{13} \mathrm{C}\left\{{ }^{1} \mathbf{H}\right\} \mathbf{N M R}\left(101 \mathrm{MHz}, \mathrm{CDCl}_{3}\right) \delta 161.1,148.4,147.8,141.8,136.9,135.5,133.0$, 130.1, 129.6, 128.9, 127.6, 126.6, 126.5, 124.3, 122.7, 29.9, 13.7; HRMS (ESI-TOF) calcd for $\mathrm{C}_{17} \mathrm{H}_{15} \mathrm{~N}_{2} \mathrm{O}_{2}{ }^{+}$ $[\mathrm{M}+\mathrm{H}]^{+} 279.1128$, found 279.1124 . 


\section{2,6-dimethyl-3-phenylquinoline (3j):}<smiles>Cc1ccc2nc(C)c(-c3ccccc3)cc2c1</smiles>

$3 \mathbf{j}$

Following the general procedure II, product $\mathbf{3 j}$ was isolated in $74 \%$ yield colorless oil. 3j: $R_{\mathrm{f}}=0.5$ (silica gel, $20 \%$ EtOAc in hexanes); ${ }^{1} \mathbf{H}$ NMR $(400 \mathrm{MHz},) \quad \delta .96$ (d, $J=8.4 \mathrm{~Hz}, 1 \mathrm{H}), 7.87(\mathrm{~s}, 1 \mathrm{H}), 7.57-7.36(\mathrm{~m}, 8 \mathrm{H}), 2.65(\mathrm{~s}, 3 \mathrm{H}), 2.53(\mathrm{~s}, 3 \mathrm{H})$. Spectroscopic data are in agreement with those in the literature. ${ }^{3}$

3-(2-fluorophenyl)-2,6-dimethylquinoline (3k):<smiles>Cc1ccc2nc(C)c(-c3ccccc3F)cc2c1</smiles>

3k

Following the general procedure II, product 3k was isolated in $62 \%$ yield as a yellow viscous liquid. 3k: $R_{\mathrm{f}}=0.5$ (silica gel, $20 \%$ EtOAc in hexanes); ${ }^{1} \mathbf{H}$ NMR $\left(400 \mathrm{MHz}, \mathrm{CDCl}_{3}\right) \delta 7.98(\mathrm{~d}, J=9.1 \mathrm{~Hz}, 1 \mathrm{H}), 7.90(\mathrm{~s}, 1 \mathrm{H}), 7.58-7.53$ (m, 2H), $7.47-7.39(\mathrm{~m}, 1 \mathrm{H}), 7.32(\mathrm{td}, J=7.5,2.0 \mathrm{~Hz}, 1 \mathrm{H}), 7.29-7.23(\mathrm{~m}, 1$ H), $7.19(\mathrm{ddd}, J=9.6,8.2,1.1 \mathrm{~Hz}, 1 \mathrm{H}), 2.60(\mathrm{~d}, J=1.2 \mathrm{~Hz}, 3 \mathrm{H}), 2.53(\mathrm{~s}, 3 \mathrm{H})$;

${ }^{13} \mathbf{C}\left\{{ }^{1} \mathbf{H}\right\}$ NMR $\left(101 \mathrm{MHz}, \mathrm{CDCl}_{3}\right) \delta 159.93(\mathrm{~d}, J=246.5 \mathrm{~Hz}), 156.9,146.0,136.6,136.1,132.1,131.70(\mathrm{~d}$, $J=2.6 \mathrm{~Hz}), 130.02(\mathrm{~d}, J=8.2 \mathrm{~Hz}), 129.8,128.3,127.6(\mathrm{~d}, J=16.4 \mathrm{~Hz}), 126.7,126.5,124.5,115.92(\mathrm{~d}, J$ $=22.1 \mathrm{~Hz}), 23.9,21.7 ;{ }^{19} \mathbf{F}$ NMR $\left(376 \mathrm{MHz}, \mathrm{CDCl}_{3}\right) \delta-114.20 ; \mathbf{H R M S}(\mathrm{ESI}-\mathrm{TOF})$ calcd for $\mathrm{C}_{17} \mathrm{H}_{15} \mathrm{FN}^{+}$ $[\mathrm{M}+\mathrm{H}]^{+}$252.1183, found 252.1189.

\section{2,4-dimethyl-3-phenylquinoline (31):}<smiles>Cc1nc2ccccc2c(C)c1-c1ccccc1</smiles>

3I

Following the general procedure II, product $\mathbf{3 l}$ was isolated in $62 \%$ yield as a colorless viscous liquid. 3l: $R_{\mathrm{f}}=0.3$ (silica gel, $10 \%$ EtOAc in hexanes); ${ }^{1} \mathbf{H}$ NMR (400 MHz, $\left.\mathrm{CDCl}_{3}\right) \delta 8.07(\mathrm{dt}, J=8.4,1.0 \mathrm{~Hz}, 1 \mathrm{H}), 8.00(\mathrm{ddd}, J=8.4,1.4,0.6 \mathrm{~Hz}, 1 \mathrm{H}), 7.70(\mathrm{ddd}$, $J=8.3,6.8,1.4 \mathrm{~Hz}, 1 \mathrm{H}), 7.58-7.39(\mathrm{~m}, 4 \mathrm{H}), 7.24-7.19(\mathrm{~m}, 2 \mathrm{H}), 2.43(\mathrm{~s}, 3 \mathrm{H}), 2.40$

(s, $3 \mathrm{H})$. Spectroscopic data are in agreement with those in the literature. ${ }^{4}$

3-(3-chlorophenyl)-2,4-dimethylquinoline (3m):<smiles>Cc1nc2ccccc2c(C)c1-c1cccc(Cl)c1</smiles>

129.6, 129.4, 129.3, 127.84, 127.79, 126.7, 126.1, 124.2, 25.5, 16.1; HRMS (ESI-TOF) calcd for $\mathrm{C}_{17} \mathrm{H}_{15} \mathrm{ClN}^{+}[\mathrm{M}+\mathrm{H}]^{+}$268.0888, found 268.0895. 


\section{2,4-dimethyl-3-(pyridin-4-yl)quinoline (3n):}

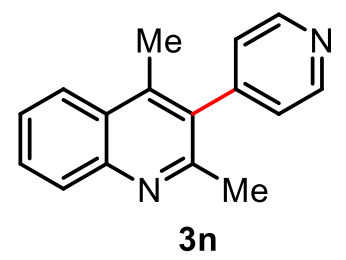

Following the general procedure II,* product 3n was isolated in $27 \%$ yield as a yellow solid. 3n: $R_{\mathrm{f}}=0.4$ (silica gel, $20 \%$ EtOAc in hexanes); ${ }^{1} \mathbf{H}$ NMR (400 MHz, $\left.\mathrm{CDCl}_{3}\right) \delta 8.76(\mathrm{~d}, J=4.9 \mathrm{~Hz}, 2 \mathrm{H}), 8.11-8.04(\mathrm{~m}, 1 \mathrm{H}), 8.01(\mathrm{dt}, J=8.4,0.9 \mathrm{~Hz}$, $1 \mathrm{H}), 7.73(\mathrm{ddd}, J=8.4,6.9,1.4 \mathrm{~Hz}, 1 \mathrm{H}), 7.57(\mathrm{ddd}, J=8.3,6.9,1.3 \mathrm{~Hz}, 1 \mathrm{H}), 7.23$ - 7.15 (m, 2H), 2.42 (s, 3H), $2.40(\mathrm{~s}, 3 \mathrm{H}) ;{ }^{13} \mathbf{C}\left\{{ }^{1} \mathbf{H}\right\} \mathbf{N M R}\left(101 \mathrm{MHz}, \mathrm{CDCl}_{3}\right) \delta$ 156.1, 150.5, 148.1 147.0, 141.2, 132.4, 129.6, 129.4, 126.5, 126.3, 124.8, 124.2, 25.4, 16.1; HRMS (ESITOF) calcd for $\mathrm{C}_{16} \mathrm{H}_{15} \mathrm{~N}_{2}{ }^{+}[\mathrm{M}+\mathrm{H}]^{+} 235.1230$, found 235.1238.

* 5 equivalents of corresponding halo diazirine was used.

\section{2,3-diphenylquinoline (3o):}<smiles>c1ccc(-c2cc3ccccc3nc2-c2ccccc2)cc1</smiles>

30
Following the general procedure II, product $\mathbf{3 0}$ was isolated in $68 \%$ yield as a colorless viscous liquid. 3o: $R_{\mathrm{f}}=0.4$ (silica gel, $20 \%$ EtOAc in hexanes); ${ }^{1} \mathbf{H}$ NMR (400 MHz, $\left.\mathrm{CDCl}_{3}\right) \delta 8.24-8.16(\mathrm{~m}, 2 \mathrm{H}), 7.88(\mathrm{dd}, J=8.1,1.4 \mathrm{~Hz}, 1 \mathrm{H}), 7.74(\mathrm{ddd}, J=8.4,6.8,1.5$ $\mathrm{Hz}, 1 \mathrm{H}), 7.57$ (ddd, $J=8.1,6.8,1.2 \mathrm{~Hz}, 1 \mathrm{H}), 7.49-7.42$ (m, 2H), $7.34-7.21$ (m, $8 \mathrm{H})$.

\section{3-(3-fluorophenyl)-2-phenylquinoline (3p):}

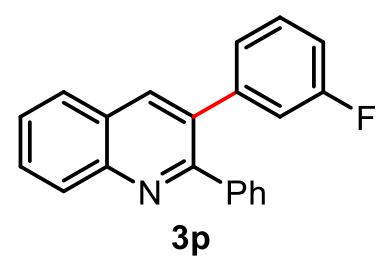

Following the general procedure II, product $\mathbf{3 p}$ was isolated in $82 \%$ yield as a yellow solid. 3p: $R_{\mathrm{f}}=0.5$ (silica gel, $20 \%$ EtOAc in hexanes); ${ }^{1} \mathbf{H}$ NMR (400 $\left.\mathrm{MHz}, \mathrm{CDCl}_{3}\right) \delta 8.21(\mathrm{~d}, J=8.5 \mathrm{~Hz}, 1 \mathrm{H}), 8.17(\mathrm{~s}, 1 \mathrm{H}), 7.88(\mathrm{dd}, J=8.2,1.4 \mathrm{~Hz}$, 1H), $7.76(\mathrm{ddd}, J=8.4,6.8,1.4 \mathrm{~Hz}, 1 \mathrm{H}), 7.58(\mathrm{td}, J=7.5,6.9,1.1 \mathrm{~Hz}, 1 \mathrm{H}), 7.49$ - $7.40(\mathrm{~m}, 2 \mathrm{H}), 7.30(\mathrm{dd}, J=5.2,1.9 \mathrm{~Hz}, 3 \mathrm{H}), 7.26-7.20(\mathrm{~m}, 1 \mathrm{H}), 7.04-6.87(\mathrm{~m}, 3 \mathrm{H}) ;{ }^{13} \mathbf{C}\left\{{ }^{1} \mathbf{H}\right\} \mathbf{~ N M R}$ $\left(101 \mathrm{MHz}, \mathrm{CDCl}_{3}\right) \delta 162.7(\mathrm{~d}, J=246.2 \mathrm{~Hz}), 158.3,147.6,142.4(\mathrm{~d}, J=7.7 \mathrm{~Hz}), 140.2,137.8,133.4(\mathrm{~d}, J$ $=2.1 \mathrm{~Hz}), 130.1,129.9,129.8,129.6,128.4,128.2,127.7,127.2,127.1,125.8(\mathrm{~d}, J=2.9 \mathrm{~Hz}), 116.8(\mathrm{~d}, J$ $=22.0 \mathrm{~Hz}), 114.3(\mathrm{~d}, J=20.9 \mathrm{~Hz}) ;{ }^{19} \mathbf{F}$ NMR $\left(376 \mathrm{MHz}, \mathrm{CDCl}_{3}\right) \delta-113.07$; HRMS (ESI-TOF) calcd for $\mathrm{C}_{21} \mathrm{H}_{15} \mathrm{FN}^{+}[\mathrm{M}+\mathrm{H}]^{+}$300.1183, found 300.1171. 


\section{3-(3-methoxyphenyl)-2-phenylquinoline (3q):}

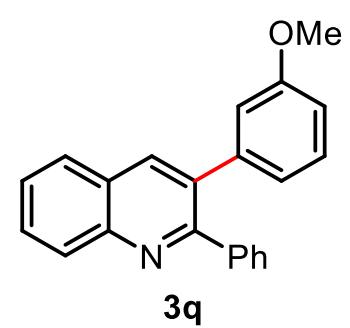

Following the general procedure II, product 3q was isolated in $73 \%$ yield as a yellow viscous liquid. 3q: $R_{\mathrm{f}}=0.4$ (silica gel, $10 \%$ EtOAc in hexanes); ${ }^{1} \mathbf{H}$ NMR $\left(400 \mathrm{MHz}, \mathrm{CDCl}_{3}\right) \delta 8.21(\mathrm{~d}, J=9.0 \mathrm{~Hz}, 2 \mathrm{H}), 7.88(\mathrm{dd}, J=8.1,1.4 \mathrm{~Hz}, 1 \mathrm{H}), 7.74$ $(\mathrm{ddd}, J=8.5,6.9,1.5 \mathrm{~Hz}, 1 \mathrm{H}), 7.57$ (ddd, $J=8.0,6.9,1.1 \mathrm{~Hz}, 1 \mathrm{H}), 7.51-7.42$ (m, $2 \mathrm{H}), 7.33-7.27(\mathrm{~m}, 3 \mathrm{H}), 7.21(\mathrm{t}, J=7.9 \mathrm{~Hz}, 1 \mathrm{H}), 6.90-6.81(\mathrm{~m}, 2 \mathrm{H}), 6.76(\mathrm{dd}, J$ = 2.6, $1.6 \mathrm{~Hz}, 1 \mathrm{H}), 3.66$ (s, 3H); ${ }^{13} \mathbf{C}\left\{{ }^{1} \mathbf{H}\right\} \mathbf{N M R}\left(101 \mathrm{MHz}, \mathrm{CDCl}_{3}\right) \delta 159.4,158.5,147.4,141.4,140.6$, $137.6,134.5,130.0,129.8,129.6,129.4,128.1$, 128.1, 127.6, 127.3, 126.9, 122.4, 115.3, 113.3, 55.3; HRMS (ESI-TOF) calcd for $\mathrm{C}_{22} \mathrm{H}_{18} \mathrm{NO}^{+}[\mathrm{M}+\mathrm{H}]^{+} 312.1383$, found 312.1390 .

\section{6-methoxy-2-methyl-3-phenylquinoline (3r):}

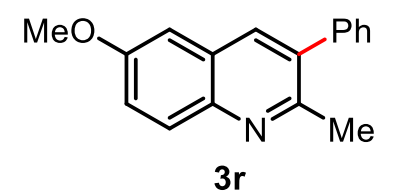

Following the general procedure II, product $3 \mathbf{r}$ was isolated in $81 \%$ yield as a yellow solid. 3r: $R_{\mathrm{f}}=0.4$ (silica gel, $10 \%$ EtOAc in hexanes); ${ }^{1} \mathbf{H}$ NMR (400 $\left.\mathrm{MHz}, \mathrm{CDCl}_{3}\right) \delta 7.96(\mathrm{~d}, J=9.2 \mathrm{~Hz}, 1 \mathrm{H}), 7.86(\mathrm{~s}, 1 \mathrm{H}), 7.50-7.38(\mathrm{~m}, 5 \mathrm{H}), 7.35$ $(\mathrm{dd}, J=9.2,2.8 \mathrm{~Hz}, 1 \mathrm{H}), 7.06(\mathrm{~d}, J=2.8 \mathrm{~Hz}, 1 \mathrm{H}), 3.92(\mathrm{~s}, 3 \mathrm{H}), 2.63(\mathrm{~s}, 3 \mathrm{H})$;

${ }^{13} \mathbf{C}\left\{{ }^{1} \mathbf{H}\right\}$ NMR $\left(101 \mathrm{MHz}, \mathrm{CDCl}_{3}\right) \delta 157.6,154.8,143.3,140.2,136.1,135.2,130.0,129.3,128.5,127.8$, 127.7, 122.1, 105.1, 55.7, 24.4; HRMS (ESI-TOF) calcd for $\mathrm{C}_{17} \mathrm{H}_{16} \mathrm{NO}^{+}[\mathrm{M}+\mathrm{H}]^{+} 250.1226$, found 250.1232 .

\section{6-methoxy-2-methyl-3-(pyrazin-2-yl)quinoline (3s):}

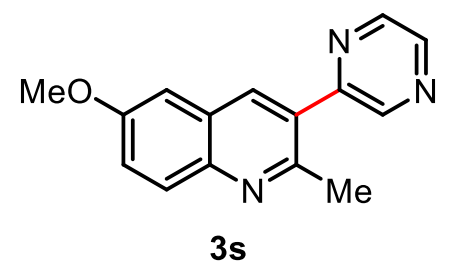

Following the general procedure II,* product $3 \mathbf{s}$ was isolated in $48 \%$ yield as a pale yellow liquid along with $29 \%$ of recovered starting material. $3 \mathbf{s}$ : $R_{\mathrm{f}}=0.3$ (silica gel, 20\% EtOAc in hexanes); ${ }^{1} \mathbf{H}$ NMR $\left(400 \mathrm{MHz}, \mathrm{CDCl}_{3}\right) \delta$ $8.84(\mathrm{~d}, J=1.5 \mathrm{~Hz}, 1 \mathrm{H}), 8.73(\mathrm{dd}, J=2.5,1.6 \mathrm{~Hz}, 1 \mathrm{H}), 8.63(\mathrm{~d}, J=2.5 \mathrm{~Hz}$, $1 \mathrm{H}), 8.11(\mathrm{~s}, 1 \mathrm{H}), 7.98(\mathrm{~d}, J=9.2 \mathrm{~Hz}, 1 \mathrm{H}), 7.41(\mathrm{dd}, J=9.2,2.8 \mathrm{~Hz}, 1 \mathrm{H})$,

$7.11(\mathrm{~d}, J=2.9 \mathrm{~Hz}, 1 \mathrm{H}), 3.94(\mathrm{~s}, 3 \mathrm{H}), 2.77(\mathrm{~s}, 3 \mathrm{H}) ;{ }^{13} \mathbf{C}\left\{{ }^{1} \mathbf{H}\right\} \mathbf{N M R}\left(101 \mathrm{MHz}, \mathrm{CDCl}_{3}\right) \delta 164.2,157.9,154.4$, 145.2, 144.2, 144.0, 143.4, 136.4, 131.1, 130.1, 127.5, 123.3, 105.4, 55.7, 24.3; HRMS (ESI-TOF) calcd for $\mathrm{C}_{15} \mathrm{H}_{14} \mathrm{~N}_{3} \mathrm{O}^{+}[\mathrm{M}+\mathrm{H}]^{+}$252.1131, found 252.1141.

* 5 equivalents of corresponding halo diazirine was used. 
2-(adamantan-1-yl)-3-(3-bromophenyl)quinoline (3t):<smiles>Brc1cccc(-c2cc3ccccc3nc2C23CC4CC(CC(C4)C2)C3)c1</smiles>

$3 \mathbf{t}$

Following the general procedure II, product $3 \mathrm{t}$ was isolated in $63 \%$ yield as a white solid. 3t: $R_{\mathrm{f}}=0.4$ (silica gel, $20 \%$ EtOAc in hexanes); ${ }^{1} \mathbf{H}$ NMR $\left(400 \mathrm{MHz}, \mathrm{CDCl}_{3}\right)$ $\delta 8.08(\mathrm{~d}, J=8.4 \mathrm{~Hz}, 1 \mathrm{H}), 7.70(\mathrm{dd}, J=16.7,8.1 \mathrm{~Hz}, 3 \mathrm{H}), 7.57-7.47(\mathrm{~m}, 3 \mathrm{H}), 7.28$ $(\mathrm{d}, J=6.8 \mathrm{~Hz}, 2 \mathrm{H}), 2.13-1.92(\mathrm{~m}, 8 \mathrm{H}), 1.74-1.44(\mathrm{~m}, 7 \mathrm{H}) ;{ }^{13} \mathbf{C}\left\{{ }^{1} \mathbf{H}\right\} \mathbf{N M R}(101$ $\left.\mathrm{MHz}, \mathrm{CDCl}_{3}\right) \delta 165.0,146.7,145.1,139.1,134.1,133.2,130.4,129.45,129.36$, 129.1, 129.0, 127.0, 126.5, 125.4, 121.7, 43.2, 42.1, 36.9, 29.1; HRMS (ESI-TOF) calcd for $\mathrm{C}_{25} \mathrm{H}_{25} \mathrm{BrN}^{+}[\mathrm{M}+\mathrm{H}]^{+}$418.1165, found 418.1173.

\section{7-(4-fluorophenyl)-6-phenyl-[1,3]dioxolo[4,5-g]quinoline (3u):}

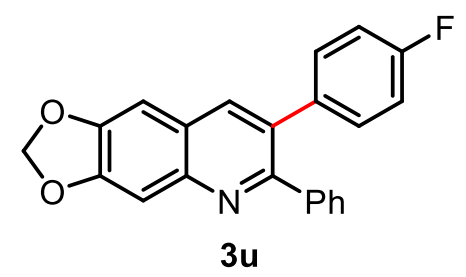

Following the general procedure II, product $\mathbf{3 u}$ was isolated in $72 \%$ yield as a yellow solid. 3u: $R_{\mathrm{f}}=0.4$ (silica gel, $10 \%$ EtOAc in hexanes); ${ }^{1} \mathbf{H}$ NMR $\left(400 \mathrm{MHz}, \mathrm{CDCl}_{3}\right) \delta 7.95(\mathrm{~s}, 1 \mathrm{H}), 7.48(\mathrm{~s}, 1 \mathrm{H}), 7.39(\mathrm{dd}, J=6.8,3.0 \mathrm{~Hz}$, 2H), $7.30-7.25$ (m, $3 \mathrm{H}), 7.21-7.12(\mathrm{~m}, 2 \mathrm{H}), 7.10$ (s, 1H), 6.97 (t, $J=8.7$ $\mathrm{Hz}, 2 \mathrm{H}), 6.13(\mathrm{~s}, 2 \mathrm{H}) ;{ }^{13} \mathbf{C}\left\{{ }^{1} \mathbf{H}\right\}$ NMR $\left.\left(101 \mathrm{MHz}, \mathrm{CDCl}_{3}\right)\right) \delta 162.2(\mathrm{~d}, J=$ $246.6 \mathrm{~Hz}), 156.1,151.1,148.3,145.8,140.5,136.6,136.3,131.8,131.5$ (d, $J=7.8 \mathrm{~Hz}), 130.1,128.2,128.0$, 124.3, $115.3(\mathrm{~d}, J=21.4 \mathrm{~Hz}), 106.0,102.5,101.9 ;{ }^{19} \mathbf{F}$ NMR $\left(376 \mathrm{MHz}, \mathrm{CDCl}_{3}\right) \delta-115.38$; HRMS (ESITOF) calcd for $\mathrm{C}_{22} \mathrm{H}_{15} \mathrm{FNO}_{2}{ }^{+}[\mathrm{M}+\mathrm{H}]^{+}$344.1081, found 344.1092.

\section{5,7-dicyclopropyl-3-(3-methoxyphenyl)-2-methylquinoline (3v):}

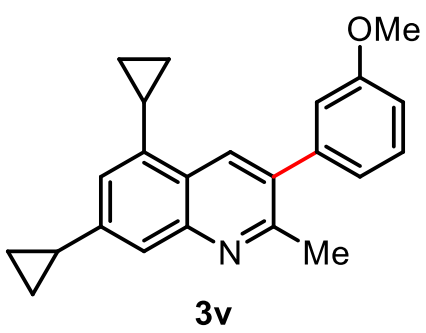

Following the general procedure II, product $3 \mathbf{v}$ was isolated in $64 \%$ yield as a pale yellow viscous liquid. 3v: $R_{\mathrm{f}}=0.4$ (silica gel, $10 \%$ EtOAc in hexanes); ${ }^{1} \mathbf{H}$ NMR $\left(400 \mathrm{MHz}, \mathrm{CDCl}_{3}\right) \delta 8.41(\mathrm{~s}, 1 \mathrm{H}), 7.53(\mathrm{~d}, J=1.6 \mathrm{~Hz}, 1 \mathrm{H}), 7.44-$ $7.37(\mathrm{~m}, 1 \mathrm{H}), 7.06(\mathrm{t}, J=1.3 \mathrm{~Hz}, 1 \mathrm{H}), 7.01(\mathrm{dt}, J=7.6,1.2 \mathrm{~Hz}, 1 \mathrm{H}), 6.97$ (dt, $J=6.8,1.6 \mathrm{~Hz}, 2 \mathrm{H}), 3.87$ (s, 3H), 2.65 (s, 3H), 2.25 (ddd, $J=13.8,8.5,5.3$ $\mathrm{Hz}, 1 \mathrm{H}), 2.03$ (tt, $J=8.3,5.1 \mathrm{~Hz}, 1 \mathrm{H}), 1.04$ (dddd, $J=12.7,8.5,6.4,4.4 \mathrm{~Hz}$, $4 \mathrm{H}), 0.86(\mathrm{dt}, J=6.8,4.6 \mathrm{~Hz}, 2 \mathrm{H}), 0.81-0.72(\mathrm{~m}, 2 \mathrm{H}) ;{ }^{13} \mathbf{C}\left\{{ }^{1} \mathbf{H}\right\} \mathbf{N M R}\left(101 \mathrm{MHz}, \mathrm{CDCl}_{3}\right) \delta 159.6,156.8$, 147.7, 145.7, 142.0, 139.3, 134.2, 133.0, 129.6, 125.3, 123.5, 122.0, 121.4, 115.5, 112.8, 55.5, 24.4, 16.1, 12.7, 10.0, 6.8; HRMS (ESI-TOF) calcd for $\mathrm{C}_{23} \mathrm{H}_{24} \mathrm{NO}^{+}[\mathrm{M}+\mathrm{H}]^{+} 330.1852$, found 330.1862. 


\section{3-(4-fluorophenyl)-2-phenyl-4H,6H-pyrano[3,4,5-de]quinoline (3w):}

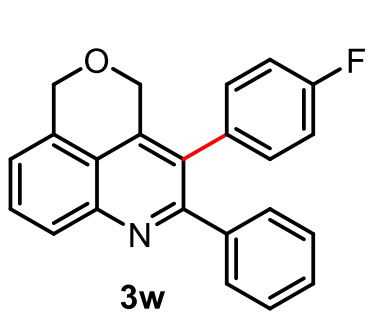

Following the general procedure II, product $3 \mathrm{w}$ was isolated in $54 \%$ yield as a yellow viscous liquid. 3w: $R_{\mathrm{f}}=0.5$ (silica gel, $20 \%$ EtOAc in hexanes); ${ }^{1} \mathbf{H}$ NMR $\left(400 \mathrm{MHz}, \mathrm{CDCl}_{3}\right) \delta 8.08(\mathrm{dd}, J=8.6,1.0 \mathrm{~Hz}, 1 \mathrm{H}), 7.70(\mathrm{dd}, J=8.6$, $7.0 \mathrm{~Hz}, 1 \mathrm{H}), 7.34-7.26(\mathrm{~m}, 3 \mathrm{H}), 7.24(\mathrm{dd}, J=5.1,1.9 \mathrm{~Hz}, 3 \mathrm{H}), 7.10-6.89$ $(\mathrm{m}, 4 \mathrm{H}), 5.06(\mathrm{~d}, J=1.1 \mathrm{~Hz}, 2 \mathrm{H}), 4.86(\mathrm{~s}, 2 \mathrm{H}) ;{ }^{13} \mathbf{C}\left\{{ }^{1} \mathbf{H}\right\}$ NMR $(101 \mathrm{MHz}$, $\left.\mathrm{CDCl}_{3}\right) \delta 162.3(\mathrm{~d}, J=247.6 \mathrm{~Hz}), 158.9,146.7,140.5,140.4,132.9,132.8,131.9(\mathrm{~d}, J=8.1 \mathrm{~Hz}), 130.0$, 129.6, 128.2, 128.1, 128.0 (2C), 121.1, 120.8, $115.6(\mathrm{~d}, J=21.5 \mathrm{~Hz}), 68.7,67.5 ;{ }^{19}$ F NMR (376 MHz, $\mathrm{CDCl}_{3}$ ) $\delta$-114.02; HRMS (ESI-TOF) calcd for $\mathrm{C}_{23} \mathrm{H}_{17} \mathrm{FNO}^{+}[\mathrm{M}+\mathrm{H}]^{+} 342.1289$, found 342.1294 .

methyl (S)-2-acetamido-3-(3-(3-nitrophenyl)quinolin-4-yl)propanoate (3x):<smiles>CC(=O)N[C@@H](Cc1c(-c2cccc([N+](=O)[O-])c2)cnc2ccccc12)C(C)=O</smiles>

$3 \mathbf{x}$

Following the general procedure II, ${ }^{*}$ product $\mathbf{3 x}$ was isolated in $41 \%$ yield as a pale yellow solid. 3x: $R_{\mathrm{f}}=0.6$ (silica gel, $50 \%$ EtOAc in hexanes); ${ }^{1} \mathbf{H}$ NMR $\left(400 \mathrm{MHz}, \mathrm{CDCl}_{3}\right) \delta 8.73(\mathrm{~s}, 1 \mathrm{H}), 8.40-8.30(\mathrm{~m}, 2 \mathrm{H}), 8.25(\mathrm{p}, J=$ $1.0 \mathrm{~Hz}, 1 \mathrm{H}), 8.19$ (dd, $J=8.4,1.3 \mathrm{~Hz}, 1 \mathrm{H}), 7.80$ (ddd, $J=8.4,6.8,1.4 \mathrm{~Hz}$, $1 \mathrm{H}), 7.76-7.68(\mathrm{~m}, 3 \mathrm{H}), 5.87(\mathrm{~d}, J=8.0 \mathrm{~Hz}, 1 \mathrm{H}), 4.83(\mathrm{q}, J=7.6 \mathrm{~Hz}, 1 \mathrm{H})$, $3.63(\mathrm{dd}, J=13.8,7.5 \mathrm{~Hz}, 1 \mathrm{H}), 3.55-3.46(\mathrm{~m}, 4 \mathrm{H}), 1.82(\mathrm{~s}, 3 \mathrm{H}) ;{ }^{13} \mathbf{C}\left\{{ }^{1} \mathbf{H}\right\} \mathbf{N M R}\left(101 \mathrm{MHz}, \mathrm{CDCl}_{3}\right) \delta$ 171.6, 169.7, 150.6, 148.5, 148.1, 140.0, 139.7, 136.1, 133.2, 130.7, 130.1, 130.0, 128.0, 127.0, 124.8, 124.2, 123.2, 52.8, 52.6, 31.8, 23.1; HRMS (ESI-TOF) calcd for $\mathrm{C}_{21} \mathrm{H}_{20} \mathrm{~N}_{3} \mathrm{O}_{5}{ }^{+}[\mathrm{M}+\mathrm{H}]^{+}$394.1397, found 394.1405 .

* Reaction was continued for $48 \mathrm{~h}$.

\section{$1^{3}$-phenyl-1(2,4)-quinolinacycloundecaphane (3y):}

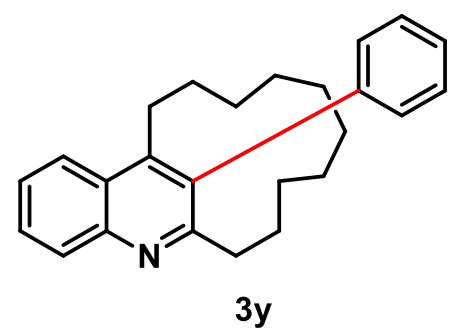

Following the general procedure II, product $\mathbf{3 y}$ was isolated in $52 \%$ yield as a colorless viscous liquid. 3y: $R_{\mathrm{f}}=0.6$ (silica gel, $20 \%$ EtOAc in hexanes); ${ }^{1} \mathbf{H}$ NMR $\left(400 \mathrm{MHz}, \mathrm{CDCl}_{3}\right) \delta 8.17-8.02(\mathrm{~m}, 2 \mathrm{H}), 7.84-7.76(\mathrm{~m}, 1 \mathrm{H})$, 7.69 (ddd, $J=8.3,6.8,1.4 \mathrm{~Hz}, 1 \mathrm{H}), 7.61-7.49(\mathrm{~m}, 2 \mathrm{H}), 7.45-7.34$ (m, 2H), $6.88(\mathrm{dt}, J=7.2,1.8 \mathrm{~Hz}, 1 \mathrm{H}), 3.24(\mathrm{dt}, J=13.7,4.2 \mathrm{~Hz}, 1 \mathrm{H}), 2.90-$ $2.80(\mathrm{~m}, 1 \mathrm{H}), 2.78-2.59(\mathrm{~m}, 2 \mathrm{H}), 2.04-1.93(\mathrm{~m}, 1 \mathrm{H}), 1.89(\mathrm{dq}, J=10.8$ 6.3, $4.7 \mathrm{~Hz}, 2 \mathrm{H}), 1.83-1.69(\mathrm{~m}, 1 \mathrm{H}), 1.65(\mathrm{~s}, 1 \mathrm{H}), 1.55-1.42(\mathrm{~m}, 1 \mathrm{H}), 1.32$ (dddd, $J=20.2,17.1,7.9,5.7$ $\mathrm{Hz}, 2 \mathrm{H}), 1.23-1.04(\mathrm{~m}, 2 \mathrm{H}), 1.04-0.83(\mathrm{~m}, 2 \mathrm{H}), 0.60(\mathrm{ttd}, J=24.1,11.7,11.0,5.5 \mathrm{~Hz}, 2 \mathrm{H}), 0.25--0.03$ $(\mathrm{m}, 2 \mathrm{H}) ;{ }^{13} \mathbf{C}\left\{{ }^{1} \mathbf{H}\right\} \mathbf{N M R}\left(101 \mathrm{MHz}, \mathrm{CDCl}_{3}\right) \delta 161.9,139.6,135.8,131.1,131.1,129.6,128.9,128.7,127.9$, 127.3, 126.3, 125.6, 124.8, 36.7, 29.8, 28.2, 28.1, 27.9, 26.9, 26.9, 26.3, 26.2, 26.2; HRMS (ESI-TOF) calcd for $\mathrm{C}_{25} \mathrm{H}_{30} \mathrm{~N}^{+}[\mathrm{M}+\mathrm{H}]^{+} 344.2373$, found 344.2381. 


\section{$1^{3}$-(4-fluorophenyl)-1(2,4)-quinolinacycloundecaphane (3z):}

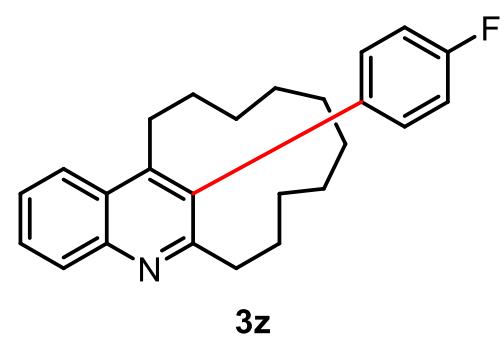

Following the general procedure II, product $3 \mathbf{z}$ was isolated in $64 \%$ yield as an off white solid. 3z: $R_{\mathrm{f}}=0.5$ (silica gel, $20 \%$ EtOAc in hexanes); ${ }^{1} \mathbf{H}$ NMR $\left(400 \mathrm{MHz}, \mathrm{CDCl}_{3}\right) \delta 8.14-8.02(\mathrm{~m}, 2 \mathrm{H}), 7.75$ (ddd, $J=8.1,5.3$, $2.4 \mathrm{~Hz}, 1 \mathrm{H}$ ), 7.70 (ddd, $J=8.3,6.8,1.3 \mathrm{~Hz}, 1 \mathrm{H}), 7.53$ (ddd, $J=8.3,6.8$, $1.3 \mathrm{~Hz}, 1 \mathrm{H}), 7.31-7.23(\mathrm{~m}, 1 \mathrm{H}), 7.07$ (td, $J=8.5,2.8 \mathrm{~Hz}, 1 \mathrm{H}), 6.85$ (ddd, $J=8.1,5.4,2.3 \mathrm{~Hz}, 1 \mathrm{H}), 3.24(\mathrm{dt}, J=13.8,4.2 \mathrm{~Hz}, 1 \mathrm{H}), 2.84(\mathrm{dt}$, $J=13.3,5.1 \mathrm{~Hz}, 1 \mathrm{H}), 2.77-2.56(\mathrm{~m}, 2 \mathrm{H}), 1.98(\mathrm{dp}, J=14.8,4.5 \mathrm{~Hz}, 1 \mathrm{H}), 1.93-1.82(\mathrm{~m}, 2 \mathrm{H}), 1.75$ (ddq, $J=14.9,11.4,4.6 \mathrm{~Hz}, 1 \mathrm{H}), 1.48(\mathrm{dtd}, J=13.9,10.8,6.7 \mathrm{~Hz}, 1 \mathrm{H}), 1.37-1.19(\mathrm{~m}, 4 \mathrm{H}), 1.11(\mathrm{dddd}, J=$ 26.1, 12.3, 6.4, 3.2 Hz, 1H), 0.97 (ttd, $J=16.8,10.2,8.8,5.5 \mathrm{~Hz}, 2 \mathrm{H}), 0.59$ (dddd, $J=27.5,13.5,10.7,5.6$ $\mathrm{Hz}, 2 \mathrm{H}), 0.21--0.05(\mathrm{~m}, 2 \mathrm{H}) ;{ }^{13} \mathbf{C}\left\{{ }^{1} \mathbf{H}\right\} \mathbf{N M R}\left(101 \mathrm{MHz}, \mathrm{CDCl}_{3}\right) \delta 162.2(\mathrm{~d}, J=247.1 \mathrm{~Hz}), 162.0,147.2$, 145.9, 135.5, 134.8, 133.0 (d, $J=7.6 \mathrm{~Hz}), 132.6$ (d, $J=7.9 \mathrm{~Hz}), 129.7,129.1,126.3,125.9,124.9,115.8$ $(\mathrm{d}, J=21.1 \mathrm{~Hz}), 115.0(\mathrm{~d}, J=21.4 \mathrm{~Hz}), 36.8,29.9,28.3$ (2C), 28.0, 27.0, 26.9, $26.4(2 \mathrm{C}), 26.2 ;{ }^{19} \mathbf{F}$ NMR $\left(376 \mathrm{MHz}, \mathrm{CDCl}_{3}\right) \delta-115.1$; HRMS (ESI-TOF) calcd for $\mathrm{C}_{25} \mathrm{H}_{29} \mathrm{FN}^{+}[\mathrm{M}+\mathrm{H}]^{+} 362.2279$, found 362.2283.

\section{3-(3-chlorophenyl)-2,6-dimethylpyridine (5a):}

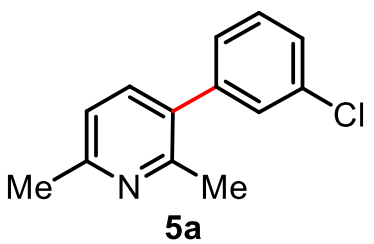

Following the general procedure II, product 5a was isolated in $73 \%$ yield as a viscous brown liquid. 5a: $R_{\mathrm{f}}=0.4$ (silica gel, $10 \%$ EtOAc in hexanes); ${ }^{1} \mathbf{H}$ NMR $\left(400 \mathrm{MHz}, \mathrm{CDCl}_{3}\right) \delta 7.39(\mathrm{~d}, J=7.7 \mathrm{~Hz}, 1 \mathrm{H}), 7.35(\mathrm{dd}, J=4.8,1.9 \mathrm{~Hz}, 2 \mathrm{H})$, $7.30(\mathrm{q}, J=1.5 \mathrm{~Hz}, 1 \mathrm{H}), 7.19$ (ddd, $J=6.2,2.9,1.7 \mathrm{~Hz}, 1 \mathrm{H}), 7.05(\mathrm{~d}, J=7.8 \mathrm{~Hz}$, 1H), $2.57(\mathrm{~s}, 3 \mathrm{H}), 2.47(\mathrm{~s}, 3 \mathrm{H}) ;{ }^{13} \mathbf{C}\left\{{ }^{1} \mathbf{H}\right\}$ NMR $\left(101 \mathrm{MHz}, \mathrm{CDCl}_{3}\right) \delta$ 157.3, 155.0, 142.1, 137.6, 134.4, 132.8, 129.8, 129.3, 127.6, 127.5, 120.7, 24.4, 23.5; HRMS (ESI-TOF) calcd for $\mathrm{C}_{13} \mathrm{H}_{13} \mathrm{ClN} \mathrm{N}^{+}[\mathrm{M}+\mathrm{H}]^{+}$ 218.0731, found 218.0731.

\section{2-methyl-3-phenyl-5,6-dihydrobenzo[ $h]$ quinoline (5b):}

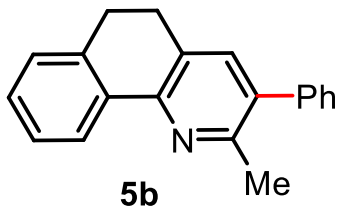

Following the general procedure II, product $\mathbf{5 b}$ was isolated in $69 \%$ yield as a yellow iol. 5b: $R_{\mathrm{f}}=0.5$ (silica gel, 20\% EtOAc in hexanes); ${ }^{1} \mathbf{H}$ NMR $(400 \mathrm{MHz}$, $\left.\mathrm{CDCl}_{3}\right) \delta 8.38(\mathrm{dd}, J=7.7,1.5 \mathrm{~Hz}, 1 \mathrm{H}), 7.45(\mathrm{ddt}, J=7.8,5.0,1.3 \mathrm{~Hz}, 2 \mathrm{H}), 7.37$ (dtd, $J=7.9,4.5,2.0 \mathrm{~Hz}, 5 \mathrm{H}), 7.31(\mathrm{td}, J=7.4,1.5 \mathrm{~Hz}, 1 \mathrm{H}), 7.24$ (dd, $J=7.4,1.4$ $\mathrm{Hz}, 1 \mathrm{H}), 2.95(\mathrm{q}, J=2.4 \mathrm{~Hz}, 4 \mathrm{H}), 2.57(\mathrm{~s}, 3 \mathrm{H})$. Spectroscopic data are in agreement with those in the literature. $^{6}$ 
3-(4-fluorophenyl)-2-methyl-5,6-diphenylpyridine (5c):<smiles>Cc1nc(-c2ccccc2)c(-c2ccccc2)cc1-c1ccc(F)cc1</smiles>

Following the general procedure II, product 5c was isolated in $63 \%$ yield as a off white solid. 5c: $R_{\mathrm{f}}=0.6$ (silica gel, $20 \%$ EtOAc in hexanes); ${ }^{1} \mathbf{H}$ NMR $(400 \mathrm{MHz}$, $\left.\mathrm{CDCl}_{3}\right) \delta 7.49(\mathrm{~s}, 1 \mathrm{H}), 7.38-7.28(\mathrm{~m}, 4 \mathrm{H}), 7.18(\mathrm{td}, J=3.5,1.8 \mathrm{~Hz}, 6 \mathrm{H}), 7.14-$ $6.99(\mathrm{~m}, 4 \mathrm{H}), 2.53(\mathrm{~s}, 3 \mathrm{H}) ;{ }^{13} \mathbf{C}\left\{{ }^{1} \mathbf{H}\right\} \mathbf{N M R}\left(101 \mathrm{MHz}, \mathrm{CDCl}_{3}\right) \delta 162.4(\mathrm{~d}, J=$ $247.0 \mathrm{~Hz}), 155.5,154.5,140.1,139.9,139.8,135.7$ (d, $J=3.5 \mathrm{~Hz}), 134.6,133.6$,

$130.9(\mathrm{~d}, J=8.1 \mathrm{~Hz}), 130.1,129.7,128.4,128.1,127.9,127.2,115.6(\mathrm{~d}, J=21.5 \mathrm{~Hz}), 23.4 ;{ }^{19} \mathbf{F} \mathbf{N M R}(376$ $\mathrm{MHz}, \mathrm{CDCl}_{3}$ ) $\delta$-114.7; HRMS (ESI-TOF) calcd for $\mathrm{C}_{24} \mathrm{H}_{19} \mathrm{FN}^{+}[\mathrm{M}+\mathrm{H}]^{+} 340.1496$, found 340.1502.

\section{ethyl 2,6-dimethyl-5-phenylnicotinate (5d):}

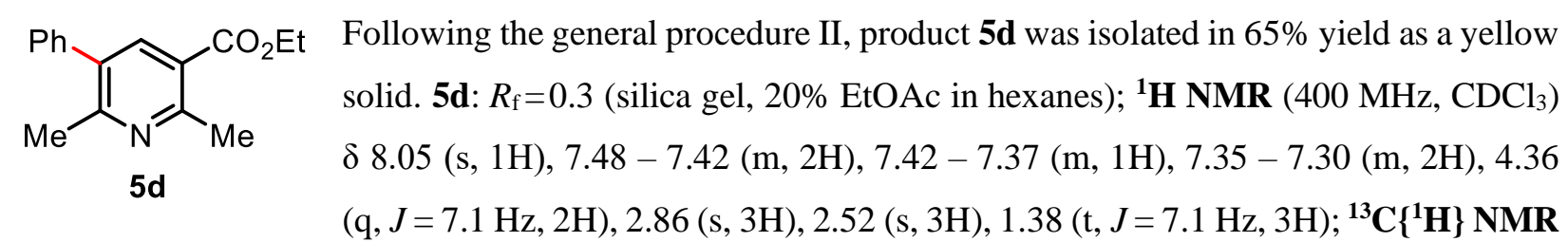

$\left(101 \mathrm{MHz}, \mathrm{CDCl}_{3}\right) \delta 166.8,158.7,158.1,139.6,139.2,134.4,129.2,128.6,127.8,123.1,61.3,24.6,23.7$, 14.4; HRMS (ESI-TOF) calcd for $\mathrm{C}_{16} \mathrm{H}_{18} \mathrm{NO}_{2}{ }^{+}[\mathrm{M}+\mathrm{H}]^{+}$256.1332, found 256.1339.

\section{3-(4-fluorophenyl)-6-(3-methoxyphenyl)-2-methyl-5-pentylpyridine (5e):}<smiles>CCCCc1cc(-c2ccc(F)cc2)c(C)nc1-c1cccc(OC)c1</smiles>

Following the general procedure II, product 5e was isolated in $46 \%$ yield as a white solid. 5e: $R_{\mathrm{f}}=0.4$ (silica gel, 10\% EtOAc in hexanes); ${ }^{1} \mathbf{H}$ NMR (400 MHz, $\left.\mathrm{CDCl}_{3}\right) \delta 7.41(\mathrm{~s}, 1 \mathrm{H}), 7.38-7.32(\mathrm{~m}, 3 \mathrm{H}), 7.19$ $-7.12(\mathrm{~m}, 2 \mathrm{H}), 7.08-7.02(\mathrm{~m}, 2 \mathrm{H}), 6.94(\mathrm{ddd}, J=8.3,2.6,1.0 \mathrm{~Hz}$, 1H), 3.85 (s, 3H), $2.67-2.58(\mathrm{~m}, 2 \mathrm{H}), 2.51$ (s, 3H), 1.52 (p, J= 7.1 $\mathrm{Hz}, 2 \mathrm{H}), 1.23$ (dtd, $J=7.2,4.8,4.1,2.1 \mathrm{~Hz}, 4 \mathrm{H}), 0.87-0.78(\mathrm{~m}, 3 \mathrm{H})$;

${ }^{13} \mathbf{C}\left\{{ }^{1} \mathbf{H}\right\}$ NMR $\left(101 \mathrm{MHz}, \mathrm{CDCl}_{3}\right) \delta 162.4(\mathrm{~d}, J=246.6 \mathrm{~Hz}), 159.6,157.2,152.6,138.7,134.7,133.1$, $130.9,130.8,129.4,121.5,115.6,115.4,114.5,113.9,55.5,32.0,31.7,30.9,23.2,22.5,14.1 .{ }^{19} \mathrm{~F}$ NMR $\left(376 \mathrm{MHz}, \mathrm{CDCl}_{3}\right) \delta-115.08$; HRMS (ESI-TOF) calcd for $\mathrm{C}_{24} \mathrm{H}_{27} \mathrm{FNO}^{+}[\mathrm{M}+\mathrm{H}]^{+}$364.2071, found 364.2080 . 
2-methyl-3,6-diphenylpyridine (5f) and 6-methyl-2,3-diphenylpyridine (5f'):<smiles>Cc1nc(-c2ccccc2)ccc1-c1ccccc1</smiles>

$5 f$

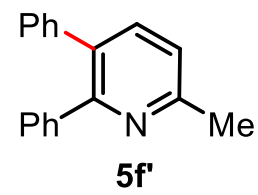

$5 f^{\prime}$

Following the general procedure II, products $\mathbf{5 f}$ and 5f' were obtained in 90\% yield in 1.9:1 ratio as a pale yellow solid and yellow viscous liquid respectively. 5f: $R_{\mathrm{f}}=0.35$ (silica gel, $10 \%$ EtOAc in hexanes); ${ }^{1} \mathbf{H}$ NMR $\left(400 \mathrm{MHz}, \mathrm{CDCl}_{3}\right) \delta 8.08-8.01(\mathrm{~m}, 2 \mathrm{H}), 7.66$ $-7.57(\mathrm{~m}, 2 \mathrm{H}), 7.52-7.43(\mathrm{~m}, 4 \mathrm{H}), 7.42-7.37(\mathrm{~m}, 4 \mathrm{H}), 2.60(\mathrm{~s}, 3 \mathrm{H})$. Spectroscopic data are in agreement with those in the literature. ${ }^{7}$

5f': $R_{\mathrm{f}}=0.3$ (silica gel, $10 \%$ EtOAc in hexanes); ${ }^{1} \mathrm{H}$ NMR $\left(400 \mathrm{MHz}, \mathrm{CDCl}_{3}\right) \delta 7.62(\mathrm{~d}, J=7.8 \mathrm{~Hz}, 1 \mathrm{H})$, $7.34(\mathrm{dd}, J=6.8,3.0 \mathrm{~Hz}, 2 \mathrm{H}), 7.25-7.10(\mathrm{~m}, 9 \mathrm{H}), 2.66(\mathrm{~s}, 3 \mathrm{H})$. Spectroscopic data are in agreement with those in the literature. ${ }^{8}$

6-(4-methoxyphenyl)-2-methyl-3-phenylpyridine phenylpyridine (5g'):<smiles>COc1ccc(-c2ccc(-c3ccccc3)c(C)n2)cc1</smiles><smiles>COc1ccc(-c2nc(C)ccc2-c2ccccc2)cc1</smiles>

(5g) and 2-(4-methoxyphenyl)-6-methyl-3Following the general procedure II, products $\mathbf{5 g}$ and $\mathbf{5 g}$ ' were obtained in $79 \%$ yield in $1.5: 1$ ratio as a white solid and pale yellow viscous liquid respectively. 5g: $R_{\mathrm{f}}=0.4$ (silica gel, $10 \%$ EtOAc in hexanes); ${ }^{1} \mathbf{H}$ NMR $\left(400 \mathrm{MHz}, \mathrm{CDCl}_{3}\right) \delta 8.05-7.96(\mathrm{~m}, 2 \mathrm{H}), 7.56(\mathrm{~s}, 2 \mathrm{H}), 7.50-7.41(\mathrm{~m}, 2 \mathrm{H}), 7.41-7.34$ $(\mathrm{m}, 3 \mathrm{H}), 7.06-6.96(\mathrm{~m}, 2 \mathrm{H}), 3.87(\mathrm{~s}, 3 \mathrm{H}), 2.58(\mathrm{~s}, 3 \mathrm{H}) ;{ }^{13} \mathbf{C}\left\{{ }^{1} \mathbf{H}\right\} \mathbf{N M R}\left(101 \mathrm{MHz}, \mathrm{CDCl}_{3}\right) \delta$ 160.5, 155.6, 155.4 140.3, 138.0, 134.8, 132.2, 129.2, 128.5, 128.3, 127.4, 117.2, 114.2, 55.5, 23.9; HRMS (ESI-TOF) calcd for $\mathrm{C}_{19} \mathrm{H}_{18} \mathrm{NO}^{+}[\mathrm{M}+\mathrm{H}]^{+} 276.1383$, found 276.1382 .

5g': $R_{\mathrm{f}}=0.35$ (silica gel, 10\% EtOAc in hexanes); ${ }^{1} \mathbf{H}$ NMR $\left(400 \mathrm{MHz}, \mathrm{CDCl}_{3}\right) \delta 7.58(\mathrm{~d}, J=7.8 \mathrm{~Hz}, 1 \mathrm{H})$, $7.27(\mathrm{dtd}, J=8.3,6.1,2.7 \mathrm{~Hz}, 5 \mathrm{H}), 7.21-7.11(\mathrm{~m}, 3 \mathrm{H}), 6.76(\mathrm{~d}, J=8.7 \mathrm{~Hz}, 2 \mathrm{H}), 3.77(\mathrm{~s}, 3 \mathrm{H}), 2.65(\mathrm{~s}, 3 \mathrm{H})$; ${ }^{13} \mathbf{C}\left\{{ }^{1} \mathbf{H}\right\}$ NMR $\left(101 \mathrm{MHz}, \mathrm{CDCl}_{3}\right) \delta 159.3,157.1,156.2,140.5,139.0,133.1,132.9,131.4,129.7,128.4$, 126.9, 121.4, 113.5, 55.4, 24.6; HRMS (ESI-TOF) calcd for $\mathrm{C}_{19} \mathrm{H}_{18} \mathrm{NO}^{+}[\mathrm{M}+\mathrm{H}]^{+} 276.1383$, found 276.1378 .

4-(6-methyl-5-phenylpyridin-2-yl)benzonitrile yl)benzonitrile (5h'):<smiles>Cc1nc(-c2ccccc2)ccc1-c1ccccc1</smiles><smiles>Cc1ccc(-c2ccccc2)c(-c2ccc(C#N)cc2)n1</smiles>

(5h) and 4-(6-methyl-3-phenylpyridin-2Following the general procedure II, products $\mathbf{5 h}$ and 5h' were obtained in $73 \%$ yield in $2.1: 1$ ratio as a colorless solid and white solid respectively. 5h: $R_{\mathrm{f}}=0.4$ (silica gel, 20\% EtOAc in hexanes); ${ }^{1} \mathbf{H}$ NMR $\left(400 \mathrm{MHz}, \mathrm{CDCl}_{3}\right) \delta 8.17-8.04(\mathrm{~m}, 2 \mathrm{H}), 7.79-7.66(\mathrm{~m}, 2 \mathrm{H}), 7.60(\mathrm{~d}, J=8.1 \mathrm{~Hz}, 1 \mathrm{H}), 7.57(\mathrm{~d}, J=8.0$ 
$\mathrm{Hz}, 1 \mathrm{H}), 7.43-7.27(\mathrm{~m}, 5 \mathrm{H}), 2.53(\mathrm{~s}, 3 \mathrm{H}) ;{ }^{13} \mathbf{C}\left\{{ }^{1} \mathbf{H}\right\}$ NMR $\left(101 \mathrm{MHz}, \mathrm{CDCl}_{3}\right) \delta 156.5,153.4,143.7,139.6$, 138.3, 136.9, 132.7, 129.1, 128.7, 127.8, 127.5, 119.1, 118.4, 112.3, 23.9; HRMS (ESI-TOF) calcd for $\mathrm{C}_{19} \mathrm{H}_{15} \mathrm{~N}_{2}{ }^{+}[\mathrm{M}+\mathrm{H}]^{+}$271.1230, found 271.1234.

5h': $R_{\mathrm{f}}=0.35$ (silica gel, 20\% EtOAc in hexanes); ${ }^{\mathbf{1}} \mathbf{H}$ NMR $\left(400 \mathrm{MHz}, \mathrm{CDCl}_{3}\right) \delta 7.65(\mathrm{~d}, J=7.9 \mathrm{~Hz}, 1 \mathrm{H})$, $7.52(\mathrm{~d}, J=8.3 \mathrm{~Hz}, 2 \mathrm{H}), 7.46(\mathrm{~d}, J=8.3 \mathrm{~Hz}, 2 \mathrm{H}), 7.31-7.26(\mathrm{~m}, 3 \mathrm{H}), 7.11(\mathrm{dt}, J=5.4,2.0 \mathrm{~Hz}, 2 \mathrm{H}), 2.66$ $(\mathrm{s}, 3 \mathrm{H}) ;{ }^{13} \mathbf{C}\left\{{ }^{1} \mathbf{H}\right\}$ NMR $\left(101 \mathrm{MHz}, \mathrm{CDCl}_{3}\right) \delta 157.8,154.4,145.2,139.3,139.2,133.7,131.9,130.8,129.7$, 128.7, 127.6, 122.8, 119.1, 111.4, 24.5; HRMS (ESI-TOF) calcd for $\mathrm{C}_{19} \mathrm{H}_{15} \mathrm{~N}_{2}{ }^{+}[\mathrm{M}+\mathrm{H}]^{+} 271.1230$, found 271.1241.

3-(3-methoxyphenyl)-2-methyl-6-phenylpyridine phenylpyridine (5i'):<smiles>COc1cccc(-c2ccc(-c3ccccc3)nc2C)c1</smiles><smiles>COc1cccc(-c2ccc(C)nc2-c2ccccc2)c1</smiles>

(5i)

and 3-(3-methoxyphenyl)-6-methyl-2-

Following the general procedure II, products $5 \mathbf{i}$ and $\mathbf{5 i}$ ' were obtained in $61 \%$ yield in $2: 1$ ratio as a white solid and colorless viscous liquid respectively. 5i: $R_{\mathrm{f}}=0.6$ (silica gel, $20 \%$ EtOAc

in hexanes); ${ }^{1} \mathbf{H}$ NMR (400 MHz, $\left.\mathrm{CDCl}_{3}\right) \delta 8.10$ - $7.97(\mathrm{~m}, 2 \mathrm{H}), 7.73-7.58(\mathrm{~m}, 2 \mathrm{H}), 7.52$ - $7.44(\mathrm{~m}, 2 \mathrm{H})$, $7.44-7.33(\mathrm{~m}, 2 \mathrm{H}), 6.99-6.85(\mathrm{~m}, 3 \mathrm{H}), 3.86(\mathrm{~s}, 3 \mathrm{H}), 2.60(\mathrm{~s}, 3 \mathrm{H}) ;{ }^{13} \mathbf{C}\left\{{ }^{1} \mathbf{H}\right\} \mathbf{N M R}\left(101 \mathrm{MHz}, \mathrm{CDCl}_{3}\right) \delta$ 159.6, 155.9, 155.8, 141.5, 139.6, 138.0, 135.4, 129.6, 128.9, 128.9, 127.1, 121.7, 117.9, 115.0, 112.9, 55.5, 23.9; HRMS (ESI-TOF) calcd for $\mathrm{C}_{19} \mathrm{H}_{18} \mathrm{NO}^{+}[\mathrm{M}+\mathrm{H}]^{+}$276.1383, found 276.1389.

5i': $R_{\mathrm{f}}=0.55$ (silica gel, 20\% EtOAc in hexanes); ${ }^{1} \mathbf{H} \mathbf{N M R}\left(400 \mathrm{MHz}, \mathrm{CDCl}_{3}\right) \delta 7.63(\mathrm{~d}, J=7.8 \mathrm{~Hz}, 1 \mathrm{H})$, $7.39-7.32(\mathrm{~m}, 2 \mathrm{H}), 7.25-7.22(\mathrm{~m}, 3 \mathrm{H}), 7.20-7.13(\mathrm{~m}, 2 \mathrm{H}), 6.79-6.73(\mathrm{~m}, 2 \mathrm{H}), 6.66(\mathrm{dd}, J=2.6,1.6$ $\mathrm{Hz}, 1 \mathrm{H}), 3.63$ (s, 3H), 2.66 (s, 3H); ${ }^{13} \mathbf{C}\left\{{ }^{1} \mathbf{H}\right\}$ NMR (101 MHz, $\left.\mathrm{CDCl}_{3}\right) \delta$ 159.4, 157.3, 156.7, 141.5, 140.6, 138.7, 133.1, 130.0, 129.3, 128.1, 127.7, 122.2, 121.8, 115.2, 113.0, 55.3, 24.6; HRMS (ESI-TOF) calcd for $\mathrm{C}_{19} \mathrm{H}_{18} \mathrm{NO}^{+}[\mathrm{M}+\mathrm{H}]^{+} 276.1383$, found 276.1391 .

\section{3-(3-chlorophenyl)-2-methyl-6-phenylpyridine} phenylpyridine (5j'):<smiles>Cc1nc(-c2ccccc2)ccc1-c1cccc(Cl)c1</smiles>

$5 \mathbf{j}$<smiles>Cc1ccc(-c2cccc(Cl)c2)c(-c2ccccc2)n1</smiles>

$5 \mathbf{j}^{\prime}$ (5j) and 3-(3-chlorophenyl)-6-methyl-2Following the general procedure II, products $\mathbf{5 j}$ and $\mathbf{5 j}$ ' were obtained in $85 \%$ yield in $1.3: 1$ ratio as a colorless viscous liquid and white solid respectively. $\mathbf{5 j}: R_{\mathrm{f}}=0.5$ (silica gel, 20\% EtOAc in hexanes); ${ }^{1} \mathbf{H}$ NMR $\left(400 \mathrm{MHz}, \mathrm{CDCl}_{3}\right) \delta 8.08-7.99(\mathrm{~m}, 2 \mathrm{H}), 7.62(\mathrm{~d}, J=8.0 \mathrm{~Hz}, 1 \mathrm{H}), 7.57(\mathrm{~d}, J=8.0 \mathrm{~Hz}, 1 \mathrm{H}), 7.53-$ $7.46(\mathrm{~m}, 2 \mathrm{H}), 7.45-7.40(\mathrm{~m}, 1 \mathrm{H}), 7.40-7.34(\mathrm{~m}, 3 \mathrm{H}), 7.28-7.24(\mathrm{~m}, 1 \mathrm{H}), 2.59(\mathrm{~s}, 3 \mathrm{H}) ;{ }^{13} \mathbf{C}\left\{{ }^{1} \mathbf{H}\right\} \mathbf{~ N M R}$ 
$\left(101 \mathrm{MHz}, \mathrm{CDCl}_{3}\right) \delta 156.3,155.7,141.9,139.4,138.0,134.4,134.1,129.8,129.3,129.1,128.9,127.7$, 127.5, 127.1, 118.0, 23.8; HRMS (ESI-TOF) calcd for $\mathrm{C}_{18} \mathrm{H}_{15} \mathrm{ClN}^{+}[\mathrm{M}+\mathrm{H}]^{+}$280.0888, found 280.0897. 5j': $R_{\mathrm{f}}=0.45$ (silica gel, 20\% EtOAc in hexanes); ${ }^{1} \mathbf{H} \mathbf{N M R}\left(400 \mathrm{MHz}, \mathrm{CDCl}_{3}\right) \delta 7.60(\mathrm{~d}, J=7.8 \mathrm{~Hz}, 1 \mathrm{H})$, $7.33(\mathrm{dd}, J=6.7,3.1 \mathrm{~Hz}, 2 \mathrm{H}), 7.26-7.18(\mathrm{~m}, 6 \mathrm{H}), 7.17-7.10(\mathrm{~m}, 1 \mathrm{H}), 6.96(\mathrm{dt}, J=7.6,1.4 \mathrm{~Hz}, 1 \mathrm{H}), 2.67$ $(\mathrm{s}, 3 \mathrm{H}) ;{ }^{13} \mathbf{C}\left\{{ }^{1} \mathbf{H}\right\} \mathbf{N M R}\left(101 \mathrm{MHz}, \mathrm{CDCl}_{3}\right) \delta 157.8,156.7,142.1,140.1,138.7,134.2,131.9,130.0,129.6$, 129.5, 128.2, 128.1, 128.0, 127.2, 121.9, 24.6; HRMS (ESI-TOF) calcd for $\mathrm{C}_{18} \mathrm{H}_{15} \mathrm{ClN}^{+}[\mathrm{M}+\mathrm{H}]^{+} 280.0888$, found 280.0883 .

2-methyl-3-(3-nitrophenyl)-6-phenylpyridine (5k) and 6-methyl-3-(3-nitrophenyl)-2-phenylpyridine (5k'):<smiles>Cc1nc(-c2ccccc2)ccc1-c1cccc([N+](=O)[O-])c1</smiles>

$5 \mathbf{k}$<smiles>Cc1ccc(-c2cccc([N+](=O)[O-])c2)c(-c2ccccc2)n1</smiles>

$5 \mathbf{k}^{\prime}$
Following the general procedure II, products $\mathbf{5 k}$ and $\mathbf{5} \mathbf{k}^{\prime}$ 'were obtained in $68 \%$ yield in 1.2:1 ratio as a pale yellow solid and yellow viscous liquid respectively. 5k: $R_{\mathrm{f}}=0.3$ (silica gel, $20 \%$ EtOAc in

hexanes); ${ }^{1} \mathbf{H}$ NMR $\left(400 \mathrm{MHz}, \mathrm{CDCl}_{3}\right) \delta 8.27(\mathrm{pd}, J=2.3,1.2 \mathrm{~Hz}, 2 \mathrm{H}), 8.10-7.99(\mathrm{~m}, 2 \mathrm{H}), 7.73(\mathrm{dt}, J=$ 7.7, $1.4 \mathrm{~Hz}, 1 \mathrm{H}), 7.69-7.64(\mathrm{~m}, 2 \mathrm{H}), 7.64-7.60(\mathrm{~m}, 1 \mathrm{H}), 7.54-7.47$ (m, 2H), $7.47-7.40(\mathrm{~m}, 1 \mathrm{H}), 2.60$ $(\mathrm{s}, 3 \mathrm{H}) ;{ }^{13} \mathbf{C}\left\{{ }^{1} \mathbf{H}\right\} \mathbf{N M R}\left(101 \mathrm{MHz}, \mathrm{CDCl}_{3}\right) \delta 156.9,155.6,148.5,141.8,139.1,138.1,135.3,133.0,129.6$, 129.3, 129.0, 127.2, 124.2, 122.6, 118.2, 23.8; HRMS (ESI-TOF) calcd for $\mathrm{C}_{18} \mathrm{H}_{15} \mathrm{~N}_{2} \mathrm{O}_{2}{ }^{+}[\mathrm{M}+\mathrm{H}]^{+} 291.1128$, found 291.1137.

5k': $R_{\mathrm{f}}=0.25$ (silica gel, 20\% EtOAc in hexanes); ${ }^{1} \mathbf{H}$ NMR $\left(400 \mathrm{MHz}, \mathrm{CDCl}_{3}\right) \delta 8.10(\mathrm{dd}, J=6.1,2.1 \mathrm{~Hz}$, 2H), $7.66(\mathrm{~d}, J=7.8 \mathrm{~Hz}, 1 \mathrm{H}), 7.42-7.36(\mathrm{~m}, 2 \mathrm{H}), 7.33-7.28(\mathrm{~m}, 2 \mathrm{H}), 7.27-7.22(\mathrm{~m}, 4 \mathrm{H}), 2.69$ (s, 3H); ${ }^{13} \mathbf{C}\left\{{ }^{1} \mathbf{H}\right\}$ NMR $\left(101 \mathrm{MHz}, \mathrm{CDCl}_{3}\right) \delta 158.6,156.9,148.4,142.0,139.6,138.7,136.0,130.9,130.0,129.2$, 128.4, 128.3, 124.4, 122.2, 122.1, 24.6; HRMS (ESI-TOF) calcd for $\mathrm{C}_{18} \mathrm{H}_{15} \mathrm{~N}_{2} \mathrm{O}_{2}{ }^{+}[\mathrm{M}+\mathrm{H}]^{+} 291.1128$, found 291.1141.

2-methyl-5-phenylpyridine (5l) and 2-methyl-3-phenylpyridine (5l'):<smiles>Cc1ccc(-c2ccccc2)cn1</smiles>

5 I<smiles>Cc1ncccc1-c1ccccc1</smiles>

$5 I^{\prime}$

Following the general procedure II, products $\mathbf{5 l}$ and 5l' were obtained in $34 \%$ yield in 2.4:1 ratio respectively as a pale-yellow liquid. 5l: $R_{\mathrm{f}}=0.4$ (silica gel, 20\% EtOAc in hexanes); ${ }^{1} \mathbf{H}$ NMR $(400 \mathrm{MHz}$, $\left.\mathrm{CDCl}_{3}\right) \delta 8.73(\mathrm{dd}, J=2.5,0.8 \mathrm{~Hz}, 1 \mathrm{H}), 7.75(\mathrm{dd}, J=8.0,2.4 \mathrm{~Hz}$, $1 \mathrm{H}), 7.55(\mathrm{~d}, \mathrm{~J}=7.6 \mathrm{~Hz}, 2 \mathrm{H}), 7.457 .20(\mathrm{~m}, 4 \mathrm{H}), 2.59(\mathrm{~s}, 3 \mathrm{H})$. Spectroscopic data are in agreement with those in the literature. ${ }^{9}$ 
5l': $R_{\mathrm{f}}=0.35$ (silica gel, 20\% EtOAc in hexanes); ${ }^{1} \mathbf{H}$ NMR $\left(400 \mathrm{MHz}, \mathrm{CDCl}_{3}\right) \delta 8.49$ (s, dd, $J=5.0,1.8$ $\mathrm{Hz}, 1 \mathrm{H}), 7.49-7.15(\mathrm{~m}, 7 \mathrm{H}), 2.50\left(3 \mathrm{H}, \mathrm{s}, \mathrm{CH}_{3}\right)$. Spectroscopic data are in agreement with those in the literature. ${ }^{10}$

2-ethyl-3,6-diphenylpyridine (5m) and 6-ethyl-2,3-diphenylpyridine (5m'):<smiles>CCc1nc(-c2ccccc2)ccc1-c1ccccc1</smiles>

$5 \mathrm{~m}$<smiles>CCc1ccc(-c2ccccc2)c(-c2ccccc2)n1</smiles>

$5 \mathrm{~m}^{\prime}$

Following the general procedure II, products $\mathbf{5 m}$ and $\mathbf{5 m}$ ' were obtained in 64\% yield in 1.1:1 ratio respectively as a white solid. $5 \mathrm{~m}$ : $R_{\mathrm{f}}=0.5$ (silica gel, 20\% EtOAc in hexanes); ${ }^{1} \mathbf{H}$ NMR $(400 \mathrm{MHz}$, $\left.\mathrm{CDCl}_{3}\right) \delta 8.15-8.02(\mathrm{~m}, 2 \mathrm{H}), 7.62(\mathrm{~d}, J=8.0 \mathrm{~Hz}, 1 \mathrm{H}), 7.56(\mathrm{~d}, J=$ $8.0 \mathrm{~Hz}, 1 \mathrm{H}), 7.52-7.33(\mathrm{~m}, 8 \mathrm{H}), 2.86(\mathrm{q}, J=7.5 \mathrm{~Hz}, 2 \mathrm{H}), 1.29(\mathrm{t}, J=7.5 \mathrm{~Hz}, 3 \mathrm{H}) ;{ }^{13} \mathbf{C}\left\{{ }^{1} \mathbf{H}\right\} \mathbf{N M R}(101$ $\left.\mathrm{MHz}, \mathrm{CDCl}_{3}\right) \delta 160.4,155.7,140.2,139.7,138.2,135.1,129.3,128.8,128.8,128.5,127.5,127.0,117.5$, 29.0, 14.0; HRMS (ESI-TOF) calcd for $\mathrm{C}_{19} \mathrm{H}_{18} \mathrm{~N}^{+}[\mathrm{M}+\mathrm{H}]^{+}$260.1434, found 260.1440. 5m': $R_{\mathrm{f}}=0.45$ (silica gel, 20\% EtOAc in hexanes); ${ }^{1} \mathbf{H}$ NMR (400 MHz, Chloroform- $d$ ) $\delta 7.64(\mathrm{~d}, J=7.9 \mathrm{~Hz}, 1 \mathrm{H}), 7.35$ (ddd, $J$ $=6.6,3.1,1.5 \mathrm{~Hz}, 2 \mathrm{H}), 7.26-7.21(\mathrm{~m}, 7 \mathrm{H}), 7.17-7.12(\mathrm{~m}, 2 \mathrm{H}), 2.94(\mathrm{q}, J=7.6 \mathrm{~Hz}, 2 \mathrm{H}), 1.39(\mathrm{t}, J=7.6$ $\mathrm{Hz}, 3 \mathrm{H}) ;{ }^{13} \mathbf{C}\left\{{ }^{1} \mathbf{H}\right\} \mathbf{N M R}\left(101 \mathrm{MHz}, \mathrm{CDCl}_{3}\right) \delta 162.4,156.5,140.7,140.3,139.0,133.4,130.1,129.7,128.3$, 128.0, 127.7, 127.0, 120.4, 31.4, 14.2; HRMS (ESI-TOF) calcd for $\mathrm{C}_{19} \mathrm{H}_{18} \mathrm{~N}^{+}[\mathrm{M}+\mathrm{H}]^{+} 260.1434$, found 260.1439 .

2-isopropyl-3,6-diphenylpyridine (5n) and 6-isopropyl-2,3-diphenylpyridine (5n'):<smiles>c1ccc(-c2ccc(-c3ccccc3)[pH]2)cc1</smiles>

$5 n$

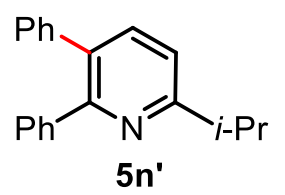

$5 n^{\prime}$

Following the general procedure II, products $5 \mathbf{n}$ and $\mathbf{5 n}$ ' were obtained in $60 \%$ yield in 1:2.6 ratio respectively as a white solid. 5n: $R_{\mathrm{f}}=0.6$ (silica gel, 20\% EtOAc in hexanes); ${ }^{1} \mathbf{H}$ NMR (400 $\left.\mathrm{MHz}, \mathrm{CDCl}_{3}\right) \delta 8.39-8.08(\mathrm{~m}, 2 \mathrm{H}), 7.61(\mathrm{~d}, J=8.0 \mathrm{~Hz}, 1 \mathrm{H}), 7.53$ $(\mathrm{d}, J=8.0 \mathrm{~Hz}, 1 \mathrm{H}), 7.51-7.38(\mathrm{~m}, 6 \mathrm{H}), 7.37-7.31(\mathrm{~m}, 2 \mathrm{H}), 3.26(\mathrm{p}, J=6.7 \mathrm{~Hz}, 1 \mathrm{H}), 1.29(\mathrm{~d}, J=6.7 \mathrm{~Hz}$, $6 \mathrm{H})$. Spectroscopic data are in agreement with those in the literature. ${ }^{11}$

5n': $R_{\mathrm{f}}=0.55$ (silica gel, 20\% EtOAc in hexanes); ${ }^{1} \mathbf{H} \mathbf{N M R}\left(400 \mathrm{MHz}, \mathrm{CDCl}_{3}\right) \delta 7.64(\mathrm{~d}, J=7.9 \mathrm{~Hz}, 1 \mathrm{H})$, $7.43-7.33(\mathrm{~m}, 2 \mathrm{H}), 7.23(\mathrm{td}, J=5.2,4.4,2.2 \mathrm{~Hz}, 7 \mathrm{H}), 7.16(\mathrm{dd}, J=7.3,2.3 \mathrm{~Hz}, 2 \mathrm{H}), 3.18(\mathrm{~h}, J=7.0 \mathrm{~Hz}$, $1 \mathrm{H}), 1.38(\mathrm{~d}, J=6.9 \mathrm{~Hz}, 6 \mathrm{H}) ;{ }^{13} \mathbf{C}\left\{{ }^{1} \mathbf{H}\right\} \mathbf{N M R}\left(101 \mathrm{MHz}, \mathrm{CDCl}_{3}\right) \delta 166.2,156.0,140.7,140.5,139.0,133.4$, 130.2, 129.7, 128.3, 127.9, 127.7, 127.0, 118.7, 36.4, 22.9; HRMS (ESI-TOF) calcd for $\mathrm{C}_{20} \mathrm{H}_{20} \mathrm{~N}^{+}[\mathrm{M}+\mathrm{H}]^{+}$ 274.1590, found 274.1594 . 
3-(4-fluorophenyl)-2-isobutyl-6-(thiophen-2-yl)pyridine (5o) and 3-(4-fluorophenyl)-6-isobutyl-2(thiophen-2-yl)pyridine (50'):
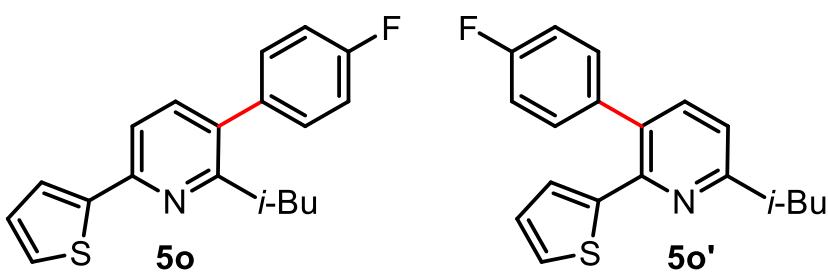

Following the general procedure II, products 50 and 5o' were obtained in 59\% yield in 1:1 ratio as a yellow solid and pale yellow solid respectively. 5o: $R_{\mathrm{f}}=0.45$ (silica gel, $20 \%$ EtOAc in hexanes); ${ }^{1} \mathbf{H}$ NMR $\left(400 \mathrm{MHz}, \mathrm{CDCl}_{3}\right) \delta 7.60(\mathrm{dd}, J=3.7$,

$1.1 \mathrm{~Hz}, 1 \mathrm{H}), 7.51(\mathrm{~d}, J=8.0 \mathrm{~Hz}, 1 \mathrm{H}), 7.46(\mathrm{~d}, J=8.0 \mathrm{~Hz}, 1 \mathrm{H}), 7.38(\mathrm{dd}, J=5.1,1.2 \mathrm{~Hz}, 1 \mathrm{H}), 7.30-7.25$ (m, 2H), $7.16-7.09(\mathrm{~m}, 3 \mathrm{H}), 2.65(\mathrm{~d}, J=7.1 \mathrm{~Hz}, 2 \mathrm{H}), 2.23(\mathrm{dp}, J=13.7,6.8 \mathrm{~Hz}, 1 \mathrm{H}), 0.83(\mathrm{~d}, J=6.6 \mathrm{~Hz}$, $6 \mathrm{H}) ;{ }^{13} \mathbf{C}\left\{{ }^{1} \mathbf{H}\right\} \mathbf{N M R}\left(101 \mathrm{MHz}, \mathrm{CDCl}_{3}\right) \delta 162.3(\mathrm{~d}, J=246.6 \mathrm{~Hz}), 158.8,151.0,145.6,138.1,136.2(\mathrm{~d}, J=$ $3.4 \mathrm{~Hz}), 134.7,131.1$ (d, $J=7.9 \mathrm{~Hz}), 128.1,127.5,124.3,115.7,115.4$ (d, $J=21.4 \mathrm{~Hz}), 43.9,28.6,22.6$; ${ }^{19}$ F NMR $\left(376 \mathrm{MHz}, \mathrm{CDCl}_{3}\right) \delta-115.17$; HRMS (ESI-TOF) calcd for $\mathrm{C}_{19} \mathrm{H}_{19} \mathrm{FNS}^{+}[\mathrm{M}+\mathrm{H}]^{+} 312.1217$, found 312.1225 .

5o': $R_{\mathrm{f}}=0.4$ (silica gel, 20\% EtOAc in hexanes); ${ }^{1} \mathbf{H}$ NMR $\left(400 \mathrm{MHz}, \mathrm{CDCl}_{3}\right) \delta 7.42(\mathrm{~d}, J=7.7 \mathrm{~Hz}, 1 \mathrm{H})$, $7.32-7.25(\mathrm{~m}, 3 \mathrm{H}), 7.15-7.07(\mathrm{~m}, 2 \mathrm{H}), 7.02(\mathrm{~d}, J=7.8 \mathrm{~Hz}, 1 \mathrm{H}), 6.81(\mathrm{dd}, J=5.1,3.7 \mathrm{~Hz}, 1 \mathrm{H}), 6.57$ (dd, $J=3.8,1.1 \mathrm{~Hz}, 1 \mathrm{H}), 2.71(\mathrm{~d}, J=7.2 \mathrm{~Hz}, 2 \mathrm{H}), 2.23(\mathrm{dp}, J=13.6,6.8 \mathrm{~Hz}, 1 \mathrm{H}), 1.01(\mathrm{~d}, J=6.6 \mathrm{~Hz}, 6 \mathrm{H})$; ${ }^{13} \mathbf{C}\left\{{ }^{1} \mathbf{H}\right\}$ NMR $\left(101 \mathrm{MHz}, \mathrm{CDCl}_{3}\right) \delta 162.6(\mathrm{~d}, J=246.9 \mathrm{~Hz}), 160.5,149.5,145.1,138.9,136.4(\mathrm{~d}, J=3.4$ $\mathrm{Hz}), 131.2(\mathrm{~d}, J=8.1 \mathrm{~Hz}), 130.9,127.6(\mathrm{~d}, J=23.9 \mathrm{~Hz}), 127.5,121.4,115.9,115.7,47.2,28.9,22.7 ;{ }^{19} \mathbf{F}$ NMR $\left(376 \mathrm{MHz}, \mathrm{CDCl}_{3}\right.$ ) $\delta-114.6$; HRMS (ESI-TOF) calcd for $\mathrm{C}_{19} \mathrm{H}_{19} \mathrm{FNS}^{+}[\mathrm{M}+\mathrm{H}]^{+} 312.1217$, found 312.1225 .

$N$-(2-(5-(4-fluorophenyl)-6-methylpyridin-2-yl)-3-methylbutyl)benzamide $\quad(5 p) \quad$ and tert-butyl benzoyl(2-(3-(4-fluorophenyl)-6-methylpyridin-2-yl)-3-methylbutyl)carbamate (5p'):
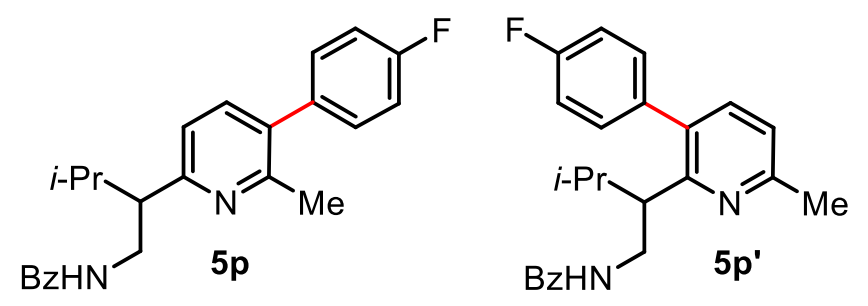

Following the general procedure II, products $\mathbf{5 p}$ and 5p' were obtained in 59\% yield in 1:3 ratio respectively as a white solid. 5p: $R_{\mathrm{f}}=0.25$ (silica gel, $40 \%$ EtOAc in hexanes); ${ }^{1} \mathbf{H}$ NMR (400 $\left.\mathrm{MHz}, \mathrm{CDCl}_{3}\right) \delta 8.25(\mathrm{~d}, J=7.0 \mathrm{~Hz}, 1 \mathrm{H}), 7.90-$ $7.70(\mathrm{~m}, 2 \mathrm{H}), 7.42-7.32(\mathrm{~m}, 4 \mathrm{H}), 7.25-7.18(\mathrm{~m}, 2 \mathrm{H}), 7.10-7.02(\mathrm{~m}, 2 \mathrm{H}), 6.98(\mathrm{~d}, J=7.7 \mathrm{~Hz}, 1 \mathrm{H}), 4.14$ (ddd, $J=13.3,7.2,5.8 \mathrm{~Hz}, 1 \mathrm{H}), 3.55-3.30(\mathrm{~m}, 1 \mathrm{H}), 2.59$ (ddd, $J=9.1,5.6,2.9 \mathrm{~Hz}, 1 \mathrm{H}), 2.45(\mathrm{~s}, 3 \mathrm{H}), 2.18$ $(\mathrm{dp}, J=9.5,6.6 \mathrm{~Hz}, 1 \mathrm{H}), 1.03(\mathrm{~d}, J=6.6 \mathrm{~Hz}, 3 \mathrm{H}), 0.72(\mathrm{~d}, J=6.6 \mathrm{~Hz}, 3 \mathrm{H}) ;{ }^{13} \mathbf{C}\left\{{ }^{1} \mathbf{H}\right\} \mathbf{N M R}(101 \mathrm{MHz}$, $\left.\mathrm{CDCl}_{3}\right) \delta 167.2,162.4(\mathrm{~d}, J=246.9 \mathrm{~Hz}), 162.1,155.1,137.9,135.8(\mathrm{~d}, J=3.2 \mathrm{~Hz}), 135.2,134.1,131.3$, $130.8(\mathrm{~d}, J=8.1 \mathrm{~Hz}), 128.6,127.1,121.6,115.6(\mathrm{~d}, J=21.3 \mathrm{~Hz}), 52.4,41.4,30.0,23.8,21.7,20.7 ;{ }^{19} \mathbf{F}$ 
NMR (376 MHz, CDC13) $\delta$-114.8; HRMS (ESI-TOF) calcd for $\mathrm{C}_{24} \mathrm{H}_{26} \mathrm{FN}_{2} \mathrm{O}^{+}[\mathrm{M}+\mathrm{H}]^{+} 377.2024$, found 377.2034 .

5p': $R_{\mathrm{f}}=0.3$ (silica gel, 40\% EtOAc in hexanes); ${ }^{1} \mathbf{H} \mathbf{N M R}\left(400 \mathrm{MHz}, \mathrm{CDCl}_{3}\right) \delta 8.72(\mathrm{~d}, J=7.1 \mathrm{~Hz}, 1 \mathrm{H})$, $8.07-7.76(\mathrm{~m}, 2 \mathrm{H}), 7.50-7.37$ (m, 4H), $7.20-7.14(\mathrm{~m}, 2 \mathrm{H}), 7.13-7.05$ (m, 3H), 4.18 (ddd, J = 13.5, 7.3, $4.5 \mathrm{~Hz}, 1 \mathrm{H}$ ), 3.35 (dt, $J=13.6,2.3 \mathrm{~Hz}, 1 \mathrm{H}), 2.79$ (ddd, $J=10.2,4.4,2.6 \mathrm{~Hz}, 1 \mathrm{H}$ ), 2.63 (s, 3H), 2.29 $(\mathrm{dp}, J=10.1,6.6 \mathrm{~Hz}, 1 \mathrm{H}), 0.92(\mathrm{~d}, J=6.7 \mathrm{~Hz}, 3 \mathrm{H}), 0.54(\mathrm{~d}, J=6.7 \mathrm{~Hz}, 3 \mathrm{H}) ;{ }^{13} \mathbf{C}\left\{{ }^{1} \mathbf{H}\right\} \mathbf{N M R}(101 \mathrm{MHz}$, $\left.\mathrm{CDCl}_{3}\right) \delta 167.1,162.3(\mathrm{~d}, J=247.0 \mathrm{~Hz}), 160.4,156.7,138.3,135.5(\mathrm{~d}, J=3.4 \mathrm{~Hz}), 135.0,134.2,131.2$, $131.0(\mathrm{~d}, J=7.9 \mathrm{~Hz}), 128.4,127.0,120.8,115.5(\mathrm{~d}, J=21.3 \mathrm{~Hz}), 46.5,41.4,30.0,24.5,21.3,20.3 ;{ }^{19} \mathbf{F}$ NMR (376 MHz, $\mathrm{CDCl}_{3}$ ) $\delta$-114.7; HRMS (ESI-TOF) calcd for $\mathrm{C}_{24} \mathrm{H}_{26} \mathrm{FN}_{2} \mathrm{O}^{+}[\mathrm{M}+\mathrm{H}]^{+} 377.2024$, found 377.2031 .

tert-butyl benzoyl(2-(5-(4-fluorophenyl)-6-methylpyridin-2-yl)-3-methylbutyl)carbamate (5q) III191) and tert-butyl benzoyl(2-(3-(4-fluorophenyl)-6-methylpyridin-2-yl)-3-methylbutyl)carbamate $\left(5 q^{\prime}\right)$ :
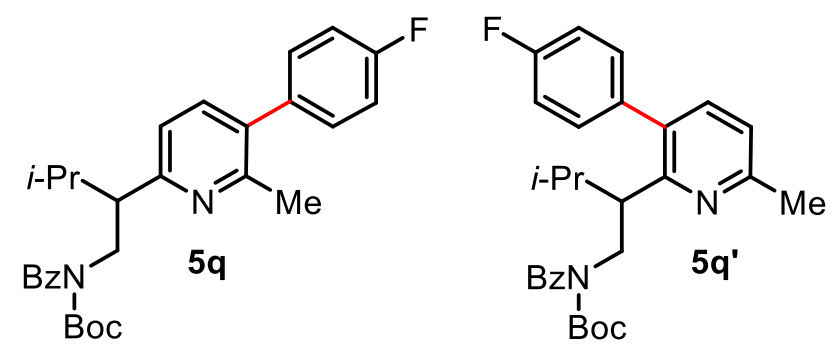

Following the general procedure II, products $\mathbf{5 q}$ and $\mathbf{5 q}$ ' were obtained in 58\% yield in 9:1 ratio as a pale yellow viscous liquid and pale yellow solid respectively. 5q: $R_{\mathrm{f}}=0.45$ (silica gel, $30 \%$ EtOAc in hexanes); ${ }^{1} \mathbf{H}$ NMR $\left(400 \mathrm{MHz}, \mathrm{CDCl}_{3}\right) \delta 7.40-$ $7.31(\mathrm{~m}, 2 \mathrm{H}), 7.22(\mathrm{t}, J=7.6 \mathrm{~Hz}, 2 \mathrm{H}), 7.15-7.02$ (m, 7H), $4.52(\mathrm{dd}, J=13.4,11.0 \mathrm{~Hz}, 1 \mathrm{H}), 4.07(\mathrm{dd}, J=13.4,4.9 \mathrm{~Hz}, 1 \mathrm{H}), 3.24(\mathrm{~d}, J=11.5 \mathrm{~Hz}, 1 \mathrm{H}), 2.29$ (s, 3H), 2.08 (dt, $J=16.4,8.1 \mathrm{~Hz}, 1 \mathrm{H}), 1.15$ (d, $J=6.7 \mathrm{~Hz}, 3 \mathrm{H}), 1.06(\mathrm{~s}, 9 \mathrm{H}), 0.85$ (d, $J=6.7 \mathrm{~Hz}, 3 \mathrm{H})$; ${ }^{13} \mathbf{C}\left\{{ }^{1} \mathbf{H}\right\}$ NMR $\left(101 \mathrm{MHz}, \mathrm{CDCl}_{3}\right) \delta 173.6,162.2(\mathrm{~d}, J=247.0 \mathrm{~Hz}), 160.6,155.1,153.5,137.9,137.1$, 136.2, 133.6, 131.0, 130.7 (d, $J=8.1 \mathrm{~Hz}), 128.2,127.9,127.8,127.7,121.4,115.3$ (d, $J=21.5 \mathrm{~Hz}), 115.2$, 82.4, 53.9, 48.4, 31.8, 29.8, 27.5, 23.2, 21.2, 20.9; ${ }^{19} \mathbf{F}$ NMR $\left(376 \mathrm{MHz}, \mathrm{CDCl}_{3}\right) \delta$-115.35; HRMS (ESITOF) calcd for $\mathrm{C}_{29} \mathrm{H}_{34} \mathrm{FN}_{2} \mathrm{O}_{3}{ }^{+}[\mathrm{M}+\mathrm{H}]^{+} 477.2548$, found 477.2548.

5q': $R_{\mathrm{f}}=0.5$ (silica gel, 30\% EtOAc in hexanes); ${ }^{1} \mathbf{H}$ NMR $\left(400 \mathrm{MHz}, \mathrm{CDCl}_{3}\right) \delta 7.42-7.35(\mathrm{~m}, 1 \mathrm{H}), 7.32$ $-7.25(\mathrm{~m}, 5 \mathrm{H}), 7.20-7.13(\mathrm{~m}, 2 \mathrm{H}), 7.09$ (t, $J=8.7 \mathrm{~Hz}, 2 \mathrm{H}), 6.89$ (d, $J=7.8 \mathrm{~Hz}, 1 \mathrm{H}), 4.58(\mathrm{dd}, J=13.2$, $9.8 \mathrm{~Hz}, 1 \mathrm{H}), 3.96$ (dd, $J=13.2,4.4 \mathrm{~Hz}, 1 \mathrm{H}), 3.44$ (ddd, $J=9.7,8.2,4.4 \mathrm{~Hz}, 1 \mathrm{H}), 2.36$ (s, 3H), $2.09-1.90$ $(\mathrm{m}, 1 \mathrm{H}), 1.02(\mathrm{~s}, 9 \mathrm{H}), 0.95(\mathrm{~d}, J=6.8 \mathrm{~Hz}, 3 \mathrm{H}), 0.65(\mathrm{~d}, J=6.7 \mathrm{~Hz}, 3 \mathrm{H}) ;{ }^{13} \mathbf{C}\left\{{ }^{1} \mathbf{H}\right\} \mathbf{N M R}\left(101 \mathrm{MHz}, \mathrm{CDCl}_{3}\right)$ $\delta 173.4,162.3(\mathrm{~d}, J=246.7 \mathrm{~Hz}), 158.7,157.3,153.7,137.9,137.7,136.3,134.8,131.5$ (d, $J=7.9 \mathrm{~Hz})$, $130.9,127.9,127.5,120.3,115.2(\mathrm{~d}, J=21.4 \mathrm{~Hz}), 82.4,48.8,48.3,32.4,27.4,24.2,21.2,20.5 ;{ }^{19} \mathbf{F}$ NMR $\left(376 \mathrm{MHz}, \mathrm{CDCl}_{3}\right) \delta-115.7$; HRMS (ESI-TOF) calcd for $\mathrm{C}_{29} \mathrm{H}_{34} \mathrm{FN}_{2} \mathrm{O}_{3}{ }^{+}[\mathrm{M}+\mathrm{H}]^{+}$477.2548, found 477.2547. 


\section{2-(4-fluorophenyl)-6-isopropyl-5-(3-nitrophenyl)- $N, 3$-diphenylisonicotinamide (5r):}

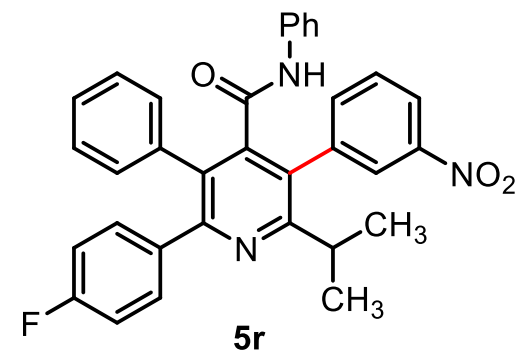

Following the general procedure II,* product $\mathbf{5 r}$ was isolated in $43 \%$ yield as a white solid, while $24 \%$ of starting material $\mathbf{4 r}$ was recovered. 5r: $R_{\mathrm{f}}=0.45$ (silica gel, 30\% EtOAc in hexanes); ${ }^{1} \mathbf{H}$ NMR $(400 \mathrm{MHz}$, $\left.\mathrm{CDCl}_{3}\right) \delta 8.31(\mathrm{t}, J=2.0 \mathrm{~Hz}, 1 \mathrm{H}), 8.26(\mathrm{ddd}, J=8.2,2.3,1.1 \mathrm{~Hz}, 1 \mathrm{H})$, $7.78(\mathrm{dt}, J=7.7,1.3 \mathrm{~Hz}, 1 \mathrm{H}), 7.62(\mathrm{t}, J=7.9 \mathrm{~Hz}, 1 \mathrm{H}), 7.42-7.35(\mathrm{~m}$, $2 \mathrm{H}), 7.30-7.25(\mathrm{~s}, 4 \mathrm{H}), 7.11(\mathrm{dd}, J=8.4,6.9 \mathrm{~Hz}, 2 \mathrm{H}), 7.03-6.98(\mathrm{~m}$, 1H), $6.95-6.86(\mathrm{~m}, 2 \mathrm{H}), 6.73-6.65(\mathrm{~m}, 3 \mathrm{H}), 2.95$ (hept, $J=6.7 \mathrm{~Hz}, 1 \mathrm{H}), 1.31(\mathrm{~d}, J=6.7 \mathrm{~Hz}, 3 \mathrm{H}), 1.26$ $(\mathrm{d}, J=6.6 \mathrm{~Hz}, 3 \mathrm{H}) ;{ }^{13} \mathbf{C}\left\{{ }^{1} \mathbf{H}\right\} \mathbf{N M R}\left(101 \mathrm{MHz}, \mathrm{CDCl}_{3}\right) \delta 165.0,163.9,162.8(\mathrm{~d}, J=248.2 \mathrm{~Hz}), 156.4,148.1$, 145.6, 138.6, 136.8, 136.0, 136.0, 135.9 (d, $J=3.1 \mathrm{~Hz}), 132.1$ (d, $J=8.2 \mathrm{~Hz}), 130.0,129.6,129.0,129.0$, $128.9,128.8,128.2,125.6,124.4,123.2,121.2,114.9(\mathrm{~d}, J=21.6 \mathrm{~Hz}), 32.5,22.6,22.5 ;{ }^{19}$ F NMR $(376$ $\mathrm{MHz}, \mathrm{CDCl}_{3}$ ) $\delta$-113.60; HRMS (ESI-TOF) calcd for $\mathrm{C}_{33} \mathrm{H}_{27} \mathrm{FN}_{3} \mathrm{O}_{3}{ }^{+}[\mathrm{M}+\mathrm{H}]^{+}$532.2031, found 532.2045. * Reaction was continued for $48 \mathrm{~h}$.

4-ethyl-3-(4-fluorophenyl)-2-methyl-6-(morpholinomethyl)-7,8-dihydroquinolin-5(6H)-one (5s):<smiles>CCc1c2c(nc(C)c1-c1ccc(F)cc1)CCC(CN1CCOCC1)C2=O</smiles>

Following the general procedure II, product 5s was isolated in $34 \%$ yield as a brown solid. 5s: $R_{\mathrm{f}}=0.3$ (silica gel, 30\% EtOAc in hexanes); ${ }^{1} \mathbf{H}$ NMR (400 MHz, $\left.\mathrm{CDCl}_{3}\right) \delta 7.21-7.04(\mathrm{~m}, 4 \mathrm{H}), 3.70$ (br. s, 5H), 3.19 (tq, $J=15.5,5.6 \mathrm{~Hz}, 2 \mathrm{H}), 2.93-2.74(\mathrm{~m}, 2 \mathrm{H}), 2.69$ $(\mathrm{dq}, J=11.9,7.3 \mathrm{~Hz}, 1 \mathrm{H}), 2.58-2.34(\mathrm{~m}, 5 \mathrm{H}), 2.21(\mathrm{~s}, 3 \mathrm{H}), 2.02-$ $1.90(\mathrm{~m}, 1 \mathrm{H}), 1.76-1.56(\mathrm{~m}, 1 \mathrm{H}), 0.96(\mathrm{t}, J=7.3 \mathrm{~Hz}, 3 \mathrm{H}) ;{ }^{13} \mathbf{C}\left\{{ }^{1} \mathbf{H}\right\} \mathbf{N M R}\left(101 \mathrm{MHz}, \mathrm{CDCl}_{3}\right) \delta 201.2$, 162.6, $162.2(\mathrm{~d}, J=247.0 \mathrm{~Hz}), 160.2,154.1,135.4,134.0,130.9$ (d, $J=8.4 \mathrm{~Hz}), 130.8,124.7,115.8(\mathrm{~d}, J$ $=21.4 \mathrm{~Hz}), 115.78(\mathrm{~d}, J=21.5 \mathrm{~Hz}), 67.0,58.4,54.0,46.5,32.2,24.7,24.0,14.8 ;{ }^{19} \mathbf{F}$ NMR $(376 \mathrm{MHz}$, $\left.\mathrm{CDCl}_{3}\right) \delta$-114.5; HRMS (ESI-TOF) calcd for $\mathrm{C}_{23} \mathrm{H}_{28} \mathrm{FN}_{2} \mathrm{O}_{2}{ }^{+}[\mathrm{M}+\mathrm{H}]^{+}$383.2129, found 383.2129.

\section{6-(tert-butyl)-2,3-diphenylpyridine (5t) and 2-(tert-butyl)-4,6-diphenylpyridine (5t'):}<smiles>CC(C)(C)c1ccc(-c2ccccc2)c(-c2ccccc2)n1</smiles>

$5 \mathbf{t}$<smiles>CC(C)(C)c1cc(-c2ccccc2)cc(-c2ccccc2)n1</smiles>

Following the general procedure II, products $\mathbf{5 t}$ and $\mathbf{5 t}$ ' were obtained in 58\% yield in 10.4:1 ratio respectively as a white solid. 5t: $R_{\mathrm{f}}=0.3$ (silica gel, 10\% EtOAc in hexanes); ${ }^{1} \mathbf{H}$ NMR $(400$ $\left.\mathrm{MHz}, \mathrm{CDCl}_{3}\right) \delta 7.55(\mathrm{dd}, J=8.0,0.9 \mathrm{~Hz}, 1 \mathrm{H}), 7.38-7.32(\mathrm{~m}$, 2H), 7.28 (d, $J=8.0 \mathrm{~Hz}, 1 \mathrm{H}), 7.22-7.09$ (m, 2H), 7.10 (dd, $J=7.4,2.2 \mathrm{~Hz}, 2 \mathrm{H}), 1.37$ (d, $J=0.9 \mathrm{~Hz}, 9 \mathrm{H})$; ${ }^{13} \mathbf{C}\left\{{ }^{1} \mathbf{H}\right\}$ NMR $\left(101 \mathrm{MHz}, \mathrm{CDCl}_{3}\right) \delta 167.9,155.1,140.9,140.7,138.8,132.8,130.4,129.7,128.4,127.7$, 127.6, 127.0, 117.4, 37.6, 30.4; HRMS (ESI-TOF) calcd for $\mathrm{C}_{21} \mathrm{H}_{22} \mathrm{~N}^{+}[\mathrm{M}+\mathrm{H}]^{+}$288.1747, found 288.1753. 
5t': $R_{\mathrm{f}}=0.6$ (silica gel, 10\% EtOAc in hexanes); ${ }^{1} \mathbf{H}$ NMR $\left(400 \mathrm{MHz}, \mathrm{CDCl}_{3}\right) \delta 8.20-8.10(\mathrm{~m}, 2 \mathrm{H}), 7.75$ $(\mathrm{d}, J=1.4 \mathrm{~Hz}, 1 \mathrm{H}), 7.71-7.65(\mathrm{~m}, 2 \mathrm{H}), 7.54-7.35(\mathrm{~m}, 7 \mathrm{H}), 1.48(\mathrm{~s}, 9 \mathrm{H})$. Spectroscopic data are in agreement with those in the literature. ${ }^{12}$

\section{2,6-di-tert-butyl-3-phenylpyridine (5u):}

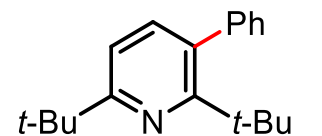

$5 \mathrm{u}$

Following the general procedure II, product $\mathbf{5 u}$ was isolated in $33 \%$ yield as a yellow viscous liquid, while $25 \%$ of starting material was recovered. $5 \mathrm{u}: R_{\mathrm{f}}=0.4$ (silica gel, $10 \%$ EtOAc in hexanes); ${ }^{1} \mathbf{H}$ NMR $\left(400 \mathrm{MHz}, \mathrm{CDCl}_{3}\right) \delta 7.36-7.31(\mathrm{~m}, 3 \mathrm{H}), 7.26-$ $7.20(\mathrm{~m}, 3 \mathrm{H}), 7.10(\mathrm{~d}, J=7.9 \mathrm{~Hz}, 1 \mathrm{H}), 1.39(\mathrm{~s}, 9 \mathrm{H}), 1.20(\mathrm{~s}, 9 \mathrm{H}) ;{ }^{13} \mathbf{C}\left\{{ }^{1} \mathbf{H}\right\} \mathbf{N M R}\left(101 \mathrm{MHz}, \mathrm{CDCl}_{3}\right) \delta$ 166.0, 162.9, 143.3, 139.9, 133.1, 130.0, 127.6, 126.9, 114.8, 40.4, 37.7, 31.7, 30.3; HRMS (ESI-TOF) calcd for $\mathrm{C}_{19} \mathrm{H}_{26} \mathrm{~N}^{+}[\mathrm{M}+\mathrm{H}]^{+}$268.2060, found 268.2058.

\section{3,4-dimethyl-5-phenylpyridine (5v) and 4,5-dimethyl-2-phenylpyridine (5v'):}<smiles>Cc1cncc(-c2ccccc2)c1C</smiles>

5v<smiles>Cc1cnc(-c2ccccc2)cc1C</smiles>

$5 v^{\prime}$

Following the general procedure II, products $\mathbf{5 v}$ and $\mathbf{5} \mathbf{v}^{\prime}$ were obtained in 58\% yield in 1.9:1 ratio respectively as a brown solid. 5v: $R_{\mathrm{f}}=0.3$ (silica gel, $30 \%$ EtOAc in hexanes); ${ }^{\mathbf{1}} \mathbf{H}$ NMR $(400$ $\left.\mathrm{MHz}, \mathrm{CDCl}_{3}\right) \delta 8.33(\mathrm{~s}, 1 \mathrm{H}), 8.29(\mathrm{~s}, 1 \mathrm{H}), 7.44(\mathrm{ddt}, J=8.1,6.5$, $1.2 \mathrm{~Hz}, 2 \mathrm{H}), 7.41-7.35(\mathrm{~m}, 1 \mathrm{H}), 7.31-7.27(\mathrm{~m}, 2 \mathrm{H}), 2.31(\mathrm{~s}, 3 \mathrm{H}), 2.18(\mathrm{~s}, 3 \mathrm{H}) ;{ }^{13} \mathbf{C}\left\{{ }^{1} \mathbf{H}\right\} \mathbf{N M R}(101 \mathrm{MHz}$, $\left.\mathrm{CDCl}_{3}\right) \delta 149.0,148.0,143.3,138.5,137.6,132.3,129.6,128.5,127.6,17.2,16.5$; HRMS (ESI-TOF) calcd for $\mathrm{C}_{13} \mathrm{H}_{14} \mathrm{~N}^{+}[\mathrm{M}+\mathrm{H}]^{+}$184.1121, found 184.1120.

5v': $R_{\mathrm{f}}=0.7$ (silica gel, 30\% EtOAc in hexanes); ${ }^{1} \mathbf{H}$ NMR $\left(400 \mathrm{MHz}, \mathrm{CDCl}_{3}\right) \delta 8.41(\mathrm{~s}, 1 \mathrm{H}), 8.00-7.91$ (m, 2H), 7.50 (s, 1H), $7.49-7.42(\mathrm{~m}, 2 \mathrm{H}), 7.42-7.35$ (m, 1H), 2.34 (s, 3H), 2.29 (s, 3H). Spectroscopic data are in agreement with those in the literature. ${ }^{13}$

Ethyl 4-ethyl-6-(4-fluorophenyl)-3,5-dimethylpicolinate (5w):<smiles>CCOC(=O)c1nc(-c2ccc(F)cc2)c(C)c(CC)c1C</smiles>

Following the general procedure II, product $\mathbf{5 w}$ was isolated in $43 \%$ yield as a viscous yellow liquid. 5w: $R_{\mathrm{f}}=0.3$ (silica gel, $30 \%$ EtOAc in hexanes); ${ }^{1} \mathbf{H}$ NMR $\left(400 \mathrm{MHz}, \mathrm{CDCl}_{3}\right) \delta 7.16-7.06(\mathrm{~m}, 4 \mathrm{H}), 4.06(\mathrm{q}, J=7.1 \mathrm{~Hz}, 2 \mathrm{H}), 2.74$ $(\mathrm{q}, J=7.6 \mathrm{~Hz}, 2 \mathrm{H}), 2.64(\mathrm{~s}, 3 \mathrm{H}), 2.07(\mathrm{~s}, 3 \mathrm{H}), 1.16(\mathrm{t}, J=7.5 \mathrm{~Hz}, 3 \mathrm{H}), 0.99$ $(\mathrm{t}, J=7.1 \mathrm{~Hz}, 3 \mathrm{H}) ;{ }^{13} \mathbf{C}\left\{{ }^{1} \mathbf{H}\right\} \mathbf{N M R}\left(101 \mathrm{MHz}, \mathrm{CDCl}_{3}\right) \delta 167.5,162.4(\mathrm{~d}, J=$ $246.7 \mathrm{~Hz}), 155.7,146.9,144.5,138.3,134.0(\mathrm{~d}, J=3.5 \mathrm{~Hz}), 133.8,131.1(\mathrm{~d}, J=7.9 \mathrm{~Hz}), 115.3(\mathrm{~d}, J=21.4$ $\mathrm{Hz}), 61.4,22.9,22.8,16.4,13.9,12.9 ;{ }^{19} \mathbf{F}$ NMR $\left(376 \mathrm{MHz}, \mathrm{CDCl}_{3}\right) \delta$-114.8; HRMS (ESI-TOF) calcd for $\mathrm{C}_{18} \mathrm{H}_{21} \mathrm{FNO}_{2}{ }^{+}[\mathrm{M}+\mathrm{H}]^{+}$302.1551, found 302.1559.

\section{Synthesis of Starting Materials}




\section{IIIA. General procedure for synthesis of chloro diazirine 1}

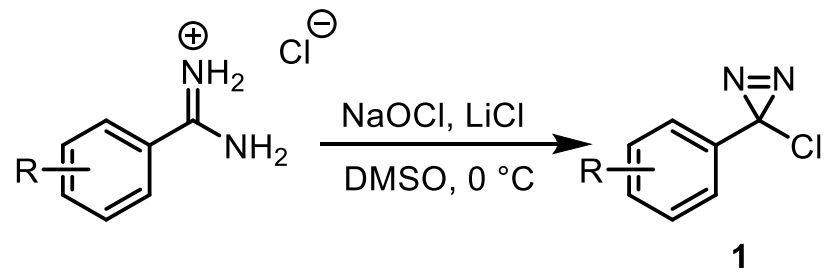

Diazerenes were prepared according to literature procedure with slight variation. ${ }^{15}$ A solution of $\mathrm{LiCl}(5.30$ g, $0.125 \mathrm{~mol})$ in DMSO $(87 \mathrm{~mL})$ was rapidly stirred within a 1-L Erlenmeyer flask bearing a ground joint. Meanwhile, $\mathrm{NaCl}(31.7 \mathrm{~g}, 0.542 \mathrm{~mol})$ was dissolved in cold $0.56 \mathrm{M} \mathrm{NaOCl}(296 \mathrm{~mL}, 0.166 \mathrm{~mol})$ in another 1-L Erlenmeyer flask. The salty chlorine bleach solution was transferred to a 500-mL dropping funnel equipped with an equilibrating sidearm and ground joints. Amidine hydrochloride hydrate $(23.7 \mathrm{mmol})$ was added to the 1-L Erlenmeyer reaction flask, and then pentane $(50 \mathrm{~mL})$ was poured in. The reaction flask was submerged into an ice bath, and rapid stirring was continued. The $\mathrm{NaOCl} / \mathrm{NaCl}$ solution was dripped in within $15 \mathrm{~min}$. The reaction mixture was stirred for an additional $30 \mathrm{~min}$ in the ice bath. The pentane layer was collected using a 1-L separatory funnel. Next, the aqueous DMSO layer was extracted with $\mathrm{Et}_{2} \mathrm{O}$ $(4 \times 25 \mathrm{~mL})$. The combined organic extracts were washed with water $(2 \times 20 \mathrm{~mL})$ and brine $(20 \mathrm{~mL})$. The organic layer was dried over anhydrous $\mathrm{MgSO}_{4}$ and then carefully rotary-evaporated. The residual oil was chromatographed using pentane (or diethyl ether/pentane) as eluant followed by careful rotary evaporation (ca. $30 \mathrm{~min}$ ) to afford pure diazerene in $20-60 \%$ yield.

\section{CAUTION! Perform behind a safety shield under dim lighting.}

\section{3-chloro-3-phenyl-3H-diazirine (1a):}

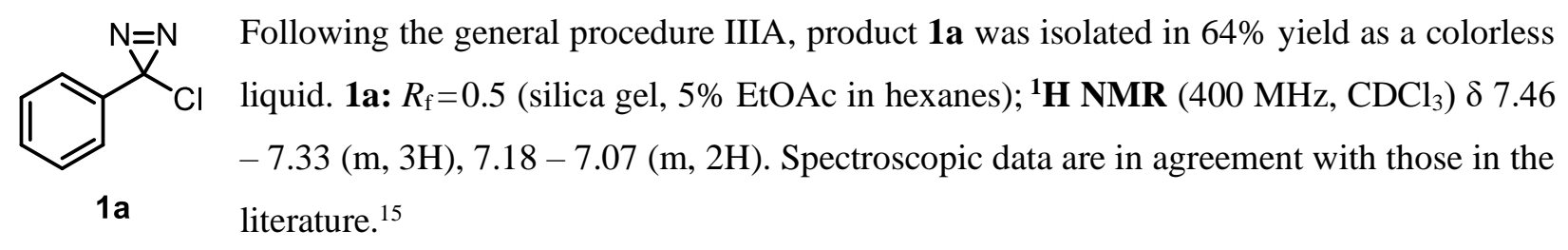

\section{2-(3-chloro-3H-diazirin-3-yl)pyridine (1b):}

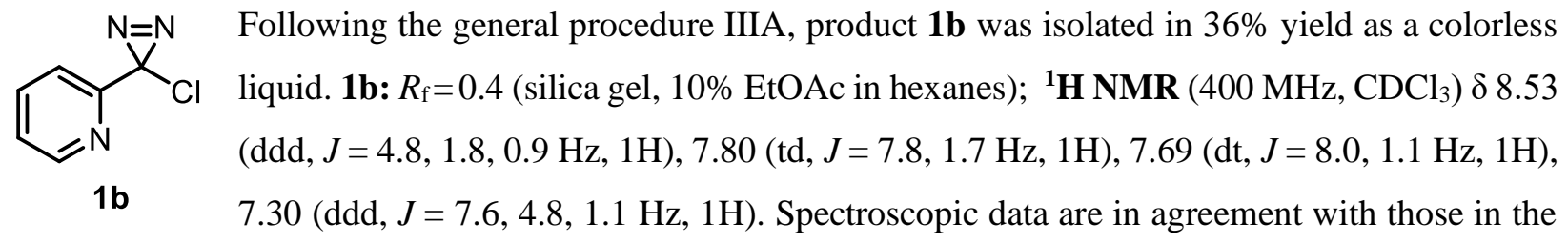
literature. ${ }^{16}$ 


\section{3-chloro-3-(4-nitrophenyl)-3H-diazirine (1c):}<smiles>O=[N+]([O-])c1ccc(C2(Cl)N=N2)cc1</smiles>

Following the general procedure IIIA, product 1c was isolated in $14 \%$ yield as a pale yellow solid. 1c: $R_{\mathrm{f}}=0.4$ (silica gel, $10 \%$ EtOAc in hexanes); ${ }^{1} \mathbf{H}$ NMR $(400 \mathrm{MHz}$, $\left.\mathrm{CDCl}_{3}\right) \delta 8.26(\mathrm{~d}, J=8.9 \mathrm{~Hz}, 2 \mathrm{H}), 7.30(\mathrm{~d}, J=9.0 \mathrm{~Hz}, 2 \mathrm{H})$. Spectroscopic data are in agreement with those in the literature. ${ }^{15}$

3-chloro-3-(3-chlorophenyl)-3H-diazirine (1d):<smiles>Clc1cccc(C2(Cl)N=N2)c1</smiles>

1d

Following the general procedure IIIA, product 1d was isolated in $61 \%$ yield as a pale-yellow liquid. 1d: $R_{\mathrm{f}}=0.4$ (silica gel, $5 \%$ EtOAc in hexanes); ${ }^{1} \mathbf{H} \mathbf{N M R}\left(400 \mathrm{MHz}, \mathrm{CDCl}_{3}\right) \delta 7.38$ (ddd, $J=8.0,2.0,1.2 \mathrm{~Hz}, 1 \mathrm{H}), 7.35-7.29(\mathrm{~m}, 1 \mathrm{H}), 7.16(\mathrm{td}, J=1.9,0.5 \mathrm{~Hz}, 1 \mathrm{H}), 6.95$ $(\mathrm{ddd}, J=7.7,1.9,1.2 \mathrm{~Hz}, 1 \mathrm{H}) ;{ }^{13} \mathbf{C}\left\{{ }^{1} \mathbf{H}\right\} \mathbf{N M R}\left(101 \mathrm{MHz}, \mathrm{CDCl}_{3}\right) \delta 137.8,135.0,129.9$, 129.7, 126.6, 124.2, 46.3; HRMS (ESI-TOF) calcd for $\mathrm{C}_{7} \mathrm{H}_{4} \mathrm{Cl}_{2}{ }^{+}\left[\mathrm{M}-\mathrm{N}_{2}\right]^{+}$157.9685, found 157.9693.

\section{3-chloro-3-(4-fluorophenyl)-3H-diazirine (1e):}

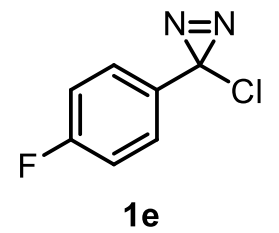

Following the general procedure IIIA, product 1e was isolated in $63 \%$ yield as a colorless liquid. 1e: $R_{\mathrm{f}}=0.4$ (silica gel, 5\% EtOAc in hexanes); ${ }^{1} \mathbf{H} \mathbf{~ N M R}(400 \mathrm{MHz}$, $\left.\mathrm{CDCl}_{3}\right) \delta 7.14-7.04(\mathrm{~m}, 4 \mathrm{H}) ;{ }^{13} \mathbf{C}\left\{{ }^{1} \mathbf{H}\right\} \mathbf{N M R}\left(101 \mathrm{MHz}, \mathrm{CDCl}_{3}\right) \delta 163.29(\mathrm{~d}, J=250.1$ Hz), 131.6, 127.95 (d, $J=8.7 \mathrm{~Hz}), 115.67$ (d, $J=22.3 \mathrm{~Hz}), 46.6 ;{ }^{19} \mathrm{~F}$ NMR $(376 \mathrm{MHz}$, $\left.\mathrm{CDCl}_{3}\right) \delta-111.45$; HRMS (ESI-TOF) calcd for $\mathrm{C}_{7} \mathrm{H}_{4} \mathrm{ClF}^{+}\left[\mathrm{M}-\mathrm{N}_{2}\right]^{+} 141.9980$, found 141.9995 .

\section{3-chloro-3-( $m$-tolyl)-3H-diazirine (1f):}

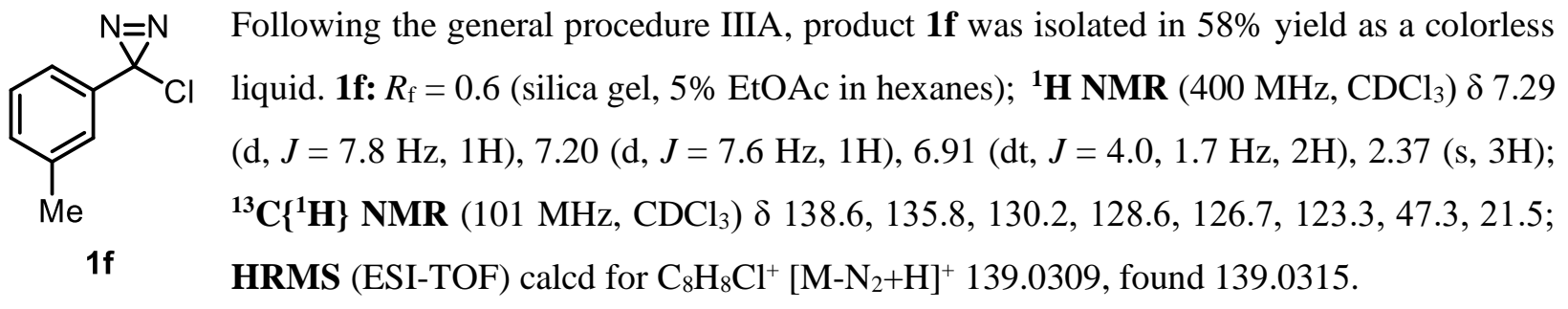

\section{3-(3-bromophenyl)-3-chloro-3H-diazirine (1g):}

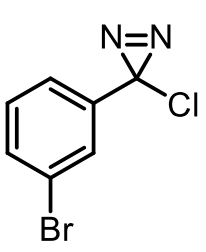

$1 \mathrm{~g}$
Following the general procedure IIIA, product $1 \mathrm{~g}$ was isolated in $55 \%$ yield as a pale yellow liquid. 1g: $R_{\mathrm{f}}=0.5$ (silica gel, 5\% EtOAc in hexanes); ${ }^{1} \mathbf{H} \mathbf{N M R}\left(400 \mathrm{MHz}, \mathrm{CDCl}_{3}\right) \delta 7.53$ $(\mathrm{ddd}, J=8.0,1.9,1.0 \mathrm{~Hz}, 1 \mathrm{H}), 7.31(\mathrm{t}, J=1.9 \mathrm{~Hz}, 1 \mathrm{H}), 7.29-7.23(\mathrm{~m}, 1 \mathrm{H}), 7.00(\mathrm{ddd}, J=$ 8.0, 1.9, $1.0 \mathrm{~Hz}, 1 \mathrm{H}) ;{ }^{13} \mathbf{C}\left\{{ }^{1} \mathbf{H}\right\} \mathbf{N M R}\left(101 \mathrm{MHz}, \mathrm{CDCl}_{3}\right) \delta 137.9,132.6,130.1,129.4,124.7$, 123.0, 46.2; HRMS (ESI-TOF) calcd for $\mathrm{C}_{7} \mathrm{H}_{4} \mathrm{BrCl}^{+}\left[\mathrm{M}-\mathrm{N}_{2}\right]^{+}$201.9185, found 201.9190. 


\section{2-(3-chloro-3H-diazirin-3-yl)pyrazine (1h):}

Folid. 1h: $R_{\mathrm{f}}=0.4\left(\right.$ silica gel, 10\% EtOAc in hexanes); ${ }^{1} \mathbf{H} \mathbf{~ N M R}\left(400 \mathrm{MHz}, \mathrm{CDCl}_{3}\right) \delta 9.02$
$(\mathrm{d}, J=1.5 \mathrm{~Hz}, 1 \mathrm{H}), 8.61(\mathrm{~d}, J=2.5 \mathrm{~Hz}, 1 \mathrm{H}), 8.49(\mathrm{dd}, J=2.5,1.5 \mathrm{~Hz}, 1 \mathrm{H}) ;{ }^{\mathbf{1 3}} \mathbf{C}\left\{{ }^{1} \mathbf{H}\right\} \mathbf{~ N M R}$ $\mathrm{C}_{5} \mathrm{H}_{3} \mathrm{ClN}_{4} \mathrm{Na}^{+}[\mathrm{M}+\mathrm{Na}]^{+}$176.9938, found 176.9938 .

\section{3-chloro-3-(2-fluorophenyl)-3H-diazirine (1i):}

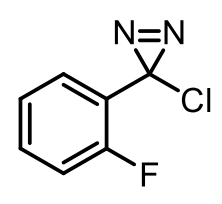

Following the general procedure IIIA, product $1 \mathbf{i}$ was isolated in $61 \%$ yield as a colorless liquid. 1i: $R_{\mathrm{f}}=0.4$ (silica gel, $5 \%$ EtOAc in hexanes); ${ }^{1} \mathbf{H}$ NMR $\left(400 \mathrm{MHz}, \mathrm{CDCl}_{3}\right) \delta 7.51$ $(\mathrm{td}, J=7.6,1.8 \mathrm{~Hz}, 1 \mathrm{H}), 7.43-7.35(\mathrm{~m}, 1 \mathrm{H}), 7.18(\mathrm{td}, J=7.6,1.2 \mathrm{~Hz}, 1 \mathrm{H}), 7.08(\mathrm{ddd}, J=$ $10.8,8.3,1.1 \mathrm{~Hz}, 1 \mathrm{H}) ;{ }^{13} \mathbf{C}\left\{{ }^{1} \mathbf{H}\right\} \mathbf{N M R}\left(101 \mathrm{MHz}, \mathrm{CDCl}_{3}\right) \delta 160.57(\mathrm{~d}, J=254.0 \mathrm{~Hz}), 132.10$ $(\mathrm{d}, J=8.2 \mathrm{~Hz}), 129.12,124.60(\mathrm{~d}, J=3.9 \mathrm{~Hz}), 122.75(\mathrm{~d}, J=11.4 \mathrm{~Hz}), 116.77(\mathrm{~d}, J=20.6 \mathrm{~Hz}), 43.06 ;{ }^{19} \mathbf{F}$ NMR $\left(376 \mathrm{MHz}, \mathrm{CDCl}_{3}\right) \delta-113.40 ;$ HRMS (ESI-TOF) calcd for $\mathrm{C} \mathrm{H}_{5} \mathrm{ClF}^{+}\left[\mathrm{M}^{-\mathrm{N}_{2}}+\mathrm{H}\right]^{+} 143.0058$, found 143.0063 .

\section{3-chloro-3-(3-methoxyphenyl)-3H-diazirine (1j):}

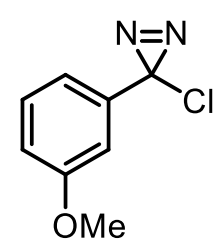

$1 \mathrm{j}$

Following the general procedure IIIA, product $\mathbf{1} \mathbf{j}$ was isolated in $64 \%$ yield as a pale yellow liquid. 1j: $R_{\mathrm{f}}=0.5$ (silica gel, 5\% EtOAc in hexanes); ${ }^{1} \mathbf{H}$ NMR $\left(400 \mathrm{MHz}, \mathrm{CDCl}_{3}\right) \delta 7.29$ (t, $J=8.0 \mathrm{~Hz}, 1 \mathrm{H}), 6.93$ (ddd, $J=8.3,2.5,0.9 \mathrm{~Hz}, 1 \mathrm{H}), 6.69$ (t, $J=2.2 \mathrm{~Hz}, 1 \mathrm{H}), 6.62$ (ddd, $J=7.8,1.9,0.9 \mathrm{~Hz}, 1 \mathrm{H}), 3.82(\mathrm{~s}, 3 \mathrm{H}) ;{ }^{13} \mathbf{C}\left\{{ }^{1} \mathbf{H}\right\} \mathbf{N M R}\left(101 \mathrm{MHz}, \mathrm{CDCl}_{3}\right) \delta 159.8,137.4$, 129.7, 118.3, 115.1, 112.0, 55.5, 47.1; HRMS (ESI-TOF) calcd for $\mathrm{C}_{8} \mathrm{H}_{7} \mathrm{ClN}_{2} \mathrm{O}^{+}[\mathrm{M}]^{+}$ 182.0241, found 182.0245 .

\section{3-chloro-3-(3-nitrophenyl)-3H-diazirine (1k):}<smiles>O=[N+]([O-])c1cccc(C2(Cl)N=N2)c1</smiles>

$\mathbf{X X}$ Following the general procedure IIIA, product $1 \mathbf{k}$ was isolated in $51 \%$ yield as a colorless liquid. 1k: $R_{\mathrm{f}}=0.4$ (silica gel, $10 \%$ EtOAc in hexanes); ${ }^{1} \mathbf{H}$ NMR $\left(400 \mathrm{MHz}, \mathrm{CDCl}_{3}\right) \delta 8.27$ (ddd, $J=8.2,2.2,1.0 \mathrm{~Hz}, 1 \mathrm{H}), 8.05(\mathrm{t}, J=2.1 \mathrm{~Hz}, 1 \mathrm{H}), 7.61(\mathrm{t}, J=8.1 \mathrm{~Hz}, 1 \mathrm{H}), 7.42$ (ddd, $J=7.9,2.0,1.0 \mathrm{~Hz}, 1 \mathrm{H}) ;{ }^{13} \mathbf{C}\left\{{ }^{1} \mathbf{H}\right\} \mathbf{N M R}\left(101 \mathrm{MHz}, \mathrm{CDCl}_{3}\right) \delta 148.5,138.0,131.6,129.9$, 124.3, 121.5, 46.0; HRMS (ESI-TOF) calcd for $\mathrm{C}_{7} \mathrm{H}_{5} \mathrm{ClNO}_{2}{ }^{+}\left[\mathrm{M}-\mathrm{N}_{2}+\mathrm{H}\right]^{+} 170.0003$, found 170.0012. 
4-chloro-3-(3-chlorophenyl)-3H-diazirine (11):<smiles>Clc1ccc(C2(Cl)N=N2)cc1</smiles>

11

Following the general procedure IIIA, product 11 was isolated in $62 \%$ yield as a colorless liquid. 11: $R_{\mathrm{f}}=0.6$ (silica gel, 5\% EtOAc in hexanes); ${ }^{1} \mathbf{H}$ NMR (400 MHz, $\left.\mathrm{CDCl}_{3}\right) \delta 7.37(\mathrm{~d}, J=8.0 \mathrm{~Hz}, 2 \mathrm{H}), 7.05(\mathrm{~d}, J=8.2 \mathrm{~Hz}, 2 \mathrm{H})$. Spectroscopic data are in agreement with those in the literature. ${ }^{15}$

\section{3-chloro-3-(3-fluorophenyl)-3H-diazirine (1m):}<smiles>Fc1cccc(C2(Cl)N=N2)c1</smiles>

Following the general procedure IIIA, product $1 \mathrm{~m}$ was isolated in $61 \%$ yield as a colorless liquid. 1m: $R_{\mathrm{f}}=0.5$ (silica gel, $5 \%$ EtOAc in hexanes); ${ }^{1} \mathbf{H}$ NMR $\left(400 \mathrm{MHz}, \mathrm{CDCl}_{3}\right) \delta 7.36$ $(\mathrm{td}, J=8.1,5.7 \mathrm{~Hz}, 1 \mathrm{H}), 7.10(\mathrm{tdd}, J=8.3,2.5,0.9 \mathrm{~Hz}, 1 \mathrm{H}), 6.92(\mathrm{dt}, J=9.7,2.2 \mathrm{~Hz}, 1 \mathrm{H})$, $6.82(\mathrm{ddd}, J=7.9,1.9,1.0 \mathrm{~Hz}, 1 \mathrm{H}) ;{ }^{13} \mathbf{C}\left\{{ }^{1} \mathbf{H}\right\} \mathbf{N M R}\left(101 \mathrm{MHz}, \mathrm{CDCl}_{3}\right) \delta 162.8(\mathrm{~d}, J=247.9$ $\mathrm{Hz}), 138.3(\mathrm{~d}, \mathrm{~J}=8.2 \mathrm{~Hz}), 130.3(\mathrm{~d}, J=8.4 \mathrm{~Hz}), 121.6(\mathrm{~d}, J=3.1 \mathrm{~Hz}), 116.6(\mathrm{~d}, J=21.2$ $\mathrm{Hz}), 113.9(\mathrm{~d}, J=24.7 \mathrm{~Hz}), 46.4 ;{ }^{19} \mathbf{F}$ NMR $\left(376 \mathrm{MHz}, \mathrm{CDCl}_{3}\right) \delta$-111.54; HRMS (ESI-TOF) calcd for $\mathrm{C}_{7} \mathrm{H}_{4} \mathrm{ClFN}_{2}{ }^{+}[\mathrm{M}]^{+}$170.0047, found 170.0019 .

3-chloro-3-(p-tolyl)-3H-diazirine (1n):<smiles>Cc1ccc(C2(Cl)N=N2)cc1</smiles>

1n

Following the general procedure IIIA, product 1n was isolated in $56 \%$ yield as a colorless liquid. 1n: $R_{\mathrm{f}}=0.6$ (silica gel, $5 \%$ EtOAc in hexanes); ${ }^{1} \mathbf{H}$ NMR $(400 \mathrm{MHz}$, $\left.\mathrm{CDCl}_{3}\right) \delta 7.22-7.16(\mathrm{~m}, 2 \mathrm{H}), 7.00(\mathrm{~d}, J=8.3 \mathrm{~Hz}, 2 \mathrm{H}), 2.38$ (s, 3H). Spectroscopic data are in agreement with those in the literature. ${ }^{17}$

\section{4-(3-chloro-3H-diazirin-3-yl)pyridine (1o):}

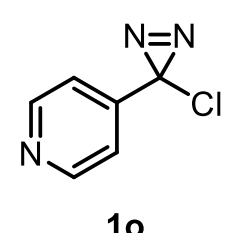

10

Following the general procedure IIIA, product 10 was isolated in 24\% yield as a colorless liquid. 1o: $R_{\mathrm{f}}=0.35$ (silica gel, $10 \%$ EtOAc in hexanes); ${ }^{1} \mathbf{H} \mathbf{N M R}\left(400 \mathrm{MHz}, \mathrm{CDCl}_{3}\right) \delta$ $8.77-8.51(\mathrm{~m}, 2 \mathrm{H}), 7.06-6.95(\mathrm{~m}, 2 \mathrm{H}) ;{ }^{13} \mathbf{C}\left\{{ }^{1} \mathbf{H}\right\} \mathbf{N M R}\left(101 \mathrm{MHz}, \mathrm{CDCl}_{3}\right) \delta 150.1,144.5$, 120.5, 45.4; HRMS (ESI-TOF) calcd for $\mathrm{C}_{6} \mathrm{H}_{4} \mathrm{ClN}_{3}{ }^{+}[\mathrm{M}+\mathrm{H}]^{+}$153.0094, found 153.0099.

\section{3-(4-bromophenyl)-3-chloro-3H-diazirine (1p):}

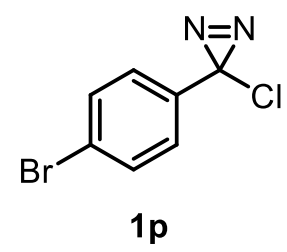

Following the general procedure IIIA, product 1p was isolated in 59\% yield as a colorless liquid. 1p: $R_{\mathrm{f}}=0.5$ (silica gel, $5 \%$ EtOAc in hexanes); ${ }^{1} \mathbf{H}$ NMR $(400 \mathrm{MHz}$, $\left.\mathrm{CDCl}_{3}\right) \delta 7.62-7.43(\mathrm{~m}, 2 \mathrm{H}), 7.05-6.90(\mathrm{~m}, 2 \mathrm{H}) ;{ }^{13} \mathbf{C}\left\{{ }^{1} \mathbf{H}\right\} \mathbf{~ N M R}\left(101 \mathrm{MHz}, \mathrm{CDCl}_{3}\right)$ $\delta 134.9,131.9,127.7,124.0,46.7$; HRMS (ESI-TOF) calcd for $\mathrm{C}_{7} \mathrm{H}_{4} \mathrm{BrCl}^{+}[\mathrm{M}+\mathrm{H}]^{+}$ 201.9179, found 201.9193. 


\section{IIIB. Synthesis of indoles and pyrroles}

\section{4,6-dicyclopropyl-2-methyl-1H-indole (2v):}

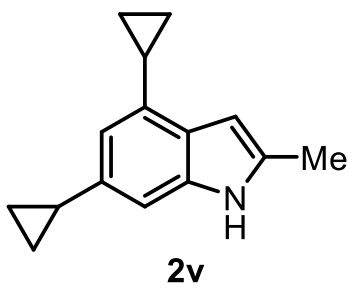

To a degassed suspension of 4,6-dichloro-2-methyl-1H-indole $(100 \mathrm{mg}, 0.50$ mmol, 1.0 equiv), cyclopropylboronic acid (171 mg, $2.0 \mathrm{mmol}, 4.0$ equiv), $\mathrm{K}_{3} \mathrm{PO}_{4}$ (690 mg, $3.0 \mathrm{mmol}, 6.0$ equiv ), tricyclohexyl phosphine ( $28 \mathrm{mg}, 0.10 \mathrm{mmol})$, and water $(0.5 \mathrm{~mL})$ in toluene $(5.0 \mathrm{~mL})$ was added palladium (II) acetate $(11 \mathrm{mg}, 50$ $\mu \mathrm{mol})$. The reaction mixture was stirred at $100{ }^{\circ} \mathrm{C}$ for $36 \mathrm{~h}$. The reaction mixture was allowed to cool to $23{ }^{\circ} \mathrm{C}$ and filtered through a pad of celite. The filtrate was concentrated under reduced pressure and the obtained residue was purified by flash column chromatography (silica gel, 2\% EtOAc in hexanes) to afford pure $\mathbf{2 v}$ ( $74 \mathrm{mg}, 0.35 \mathrm{mmol}, 70 \%$ yield) as a brown amorphous solid. $2 \mathbf{v}$ : $R_{\mathrm{f}}=0.60$ (silica gel, 10\% EtOAc in hexanes). 2v: ${ }^{1} \mathbf{H}$ NMR $\left(400 \mathrm{MHz}, \mathrm{CD}_{3} \mathrm{CN}\right) \delta 8.86(\mathrm{~s}, 1 \mathrm{H}), 6.78(\mathrm{t}, J=1.1 \mathrm{~Hz}, 1 \mathrm{H})$, $6.33(\mathrm{~d}, J=1.4 \mathrm{~Hz}, 1 \mathrm{H}), 6.19(\mathrm{dt}, J=2.2,1.1 \mathrm{~Hz}, 1 \mathrm{H}), 2.35(\mathrm{~d}, J=1.0 \mathrm{~Hz}, 3 \mathrm{H}), 2.11-2.03(\mathrm{~m}, 1 \mathrm{H}), 1.90$ - $1.81(\mathrm{~m}, 1 \mathrm{H}), 0.93-0.88(\mathrm{~m}, 2 \mathrm{H}), 0.88-0.83(\mathrm{~m}, 2 \mathrm{H}), 0.75-0.67(\mathrm{~m}, 2 \mathrm{H}), 0.64-0.56(\mathrm{~m}, 2 \mathrm{H})$; ${ }^{13} \mathbf{C}\left\{{ }^{1} \mathbf{H}\right\}$ NMR $\left(101 \mathrm{MHz}, \mathrm{CD}_{3} \mathrm{CN}\right) \delta 137.4,135.4,134.8,127.8,118.0,113.6,105.4,98.6,16.3,13.7,13.5$, 9.3, 8.0; HRMS (ESI-TOF) calcd for $\mathrm{C}_{15} \mathrm{H}_{18} \mathrm{~N}^{+}[\mathrm{M}+\mathrm{H}]^{+}$212.1434, found 212.1444.

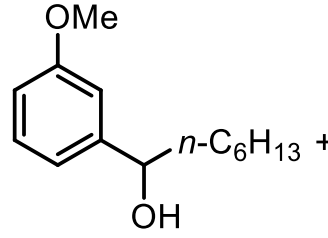

S-1<smiles>[CH2]C(N)CO</smiles>

S-2

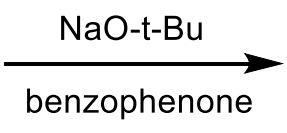

benzophenone

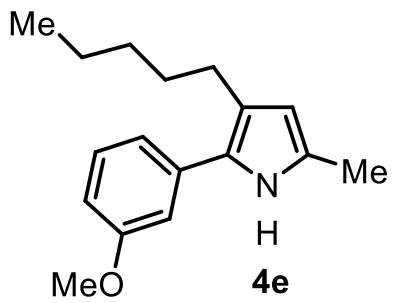

2-(3-methoxyphenyl)-5-methyl-3-pentyl-1H-pyrrole (4e) ${ }^{18}$ :

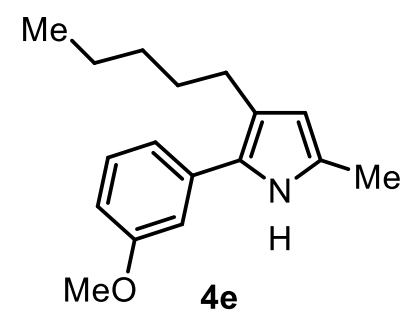

To a round-bottom flask $(10 \mathrm{~mL})$ equipped with a condenser and stirring bar were added 1-(3-methoxyphenyl)heptan-1-ol (S-1) (100 mg, $0.45 \mathrm{mmol})$, 2aminopropan-1-ol (S-2) (36 mg, $0.5 \mathrm{mmol}), \mathrm{NaO}-t$-Bu (48 mg, $0.5 \mathrm{mmol})$, benzophenone (495 mg, $2.7 \mathrm{mmol})$ in dry toluene $(6 \mathrm{~mL})$. The reaction mixture was purged with argon and refluxed $\left(110^{\circ} \mathrm{C}\right.$, oil bath $)$ overnight $(\sim 18 \mathrm{~h})$. After the reaction mixture had been cooled to room temperature, it was added directly to a silica column to afford pyrrole $4 \mathbf{e}$ (31 mg, 27\%, $0.12 \mathrm{mmol}$ ) as a yellow oil. $4 \mathbf{e}: R_{\mathrm{f}}=0.3$ (silica gel, 10\% EtOAc in hexanes); ${ }^{1} \mathbf{H}$ NMR $\left(400 \mathrm{MHz}, \mathrm{C}_{6} \mathrm{D}_{6}\right) \delta 7.20(\mathrm{~d}, J=8.0 \mathrm{~Hz}, 1 \mathrm{H}), 7.06(\mathrm{dd}, J=2.6,1.6 \mathrm{~Hz}, 1 \mathrm{H}), 6.97(\mathrm{dt}, J=7.7,1.3 \mathrm{~Hz}$, 1H), 6.72 (ddd, $J=8.3,2.6,1.0 \mathrm{~Hz}, 1 \mathrm{H}), 5.97$ (d, $J=2.9 \mathrm{~Hz}, 1 \mathrm{H}), 3.40$ (s, 3H), $2.83-2.71(\mathrm{~m}, 2 \mathrm{H}), 1.94$ $(\mathrm{d}, J=0.8 \mathrm{~Hz}, 3 \mathrm{H}), 1.76-1.70(\mathrm{~m}, 2 \mathrm{H}), 1.38-1.23(\mathrm{~m}, 4 \mathrm{H}), 0.86-0.84(\mathrm{~m}, 3 \mathrm{H}) ;{ }^{13} \mathbf{C}\left\{{ }^{1} \mathbf{H}\right\} \mathbf{~ N M R}(101$ $\left.\mathrm{MHz}, \mathrm{C}_{6} \mathrm{D}_{6}\right) \delta 160.5,136.3,129.8,127.2,126.8,122.6,119.5,113.0,111.2,109.3,54.8,32.3,31.6,27.2$, 23.1, 14.4, 13.1; HRMS (ESI-TOF) calcd for $\mathrm{C}_{17} \mathrm{H}_{24} \mathrm{~N}^{+}[\mathrm{M}+\mathrm{H}]^{+} 258.1852$, found 258.1844. 


\section{4-(5-methyl-1H-pyrrol-2-yl)benzonitrile (4h) ${ }^{19}$ :}

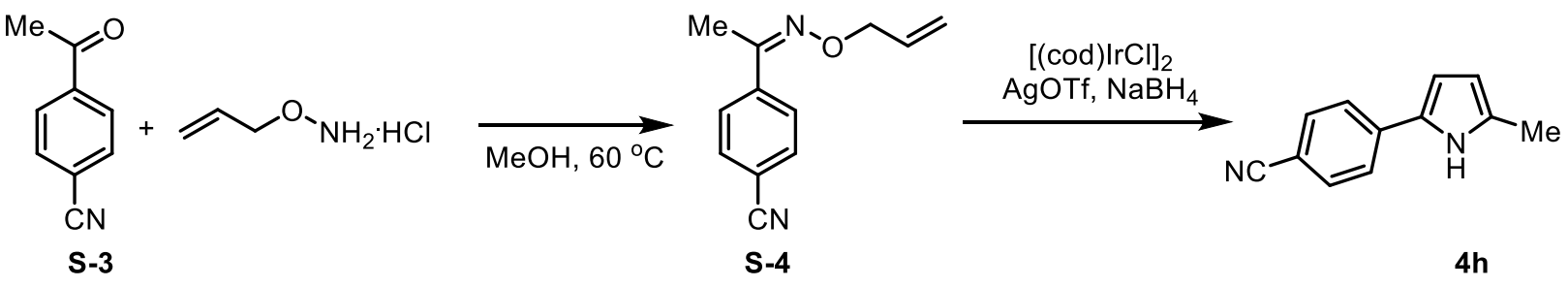

Step I- A $25 \mathrm{~mL}$ round-bottom flask was charged with allylhydroxylamine hydrochloride salt (225 mg, 2.07 mmol, 1 equiv), $\mathrm{NaOAc}(170 \mathrm{mg}, 2.07 \mathrm{mmol}, 1$ equiv) and $\mathrm{MeOH}(5 \mathrm{~mL})$. The resulting slurry was allowed to stir at $25{ }^{\circ} \mathrm{C}$ for $30 \mathrm{~min}$. At this time, 4-cyanoacetophenone $\mathbf{S - 3}$ (300 $\mathrm{mg}, 2.07 \mathrm{mmol}, 1$ equiv), was dissolved in $2 \mathrm{~mL}$ of $\mathrm{MeOH}$ and added dropwise to the reaction slurry. The reaction mixture was then allowed to stir at $60{ }^{\circ} \mathrm{C}$ for $6 \mathrm{~h}$. After completion of reaction, indicated by TLC, reaction mixture was concentrated in vacuo, $10 \mathrm{~mL}$ of water was added to the flask and extracted using EtOAc $(3 \times 10 \mathrm{~mL})$. The organic layers were then combined, dried over $\mathrm{MgSO}_{4}$, filtered and concentrated to afford $\mathbf{S}-\mathbf{4}$ (400 mg, 2 mmol) as white solid which was used for next step without purification.

Step II- In a glove box, a $20 \mathrm{~mL}$ scintillation vial was charged with [(cod) $\mathrm{IrCl}] 2(57 \mathrm{mg}, 85 \mu \mathrm{mol}, 0.05$ equiv), AgOTf (43 mg, $0.17 \mathrm{mmol}, 0.1$ equiv), $\mathrm{NaBH}_{4}(6.5 \mathrm{mg}, 0.17 \mathrm{mmol}, 0.1$ equiv), and $3 \mathrm{~mL}$ of THF. This mixture was then allowed to stir at $25{ }^{\circ} \mathrm{C}$ for $20 \mathrm{~min}$. O-Allyl oxime $\mathbf{S}-4$ (340 $\mathrm{mg}, 1.7 \mathrm{mmol}, 1$ equiv) was mixed with $2 \mathrm{~mL}$ of THF in a small vial. The oxime solution was then transferred to the scintillation vial containing the $5 \mathrm{~mol} \%$ iridium mixture and allowed to stir at $25^{\circ} \mathrm{C}$ for $24 \mathrm{~h}$. Then the reaction mixture was transferred to $10 \mathrm{~mL}$ Teflon-sealed reaction flasks, charged with $\sim 154 \AA$ molecular sieves, and heated to $75{ }^{\circ} \mathrm{C}$ for $15 \mathrm{~h}$. The reaction mixture was then transferred to scintillation vials and dry-loaded on $\sim 3 \mathrm{~mL}$ of silica gel. The product was then purified by flash chromatography to afford pyrrole $\mathbf{4 h}(120 \mathrm{mg}, 0.66$ mmol) as a brown solid. 4h: $R_{\mathrm{f}}=0.45$ (silica gel, $20 \%$ EtOAc in hexanes); ${ }^{1} \mathbf{H} \mathbf{~ N M R}\left(400 \mathrm{MHz}, \mathrm{CDCl}_{3}\right) \delta$<smiles>Cc1ccc(-c2ccc(C#N)cc2)[nH]1</smiles>

$4 h$ $8.28(\mathrm{~s}, 1 \mathrm{H}), 7.66-7.54(\mathrm{~m}, 2 \mathrm{H}), 7.52-7.41(\mathrm{~m}, 2 \mathrm{H}), 6.56(\mathrm{t}, J=3.1 \mathrm{~Hz}, 1 \mathrm{H})$, $6.01(\mathrm{ddd}, J=3.6,2.5,1.0 \mathrm{~Hz}, 1 \mathrm{H}), 2.36(\mathrm{~d}, J=0.8 \mathrm{~Hz}, 3 \mathrm{H}) ;{ }^{13} \mathbf{C}\left\{{ }^{1} \mathbf{H}\right\} \mathbf{N M R}$ $\left(101 \mathrm{MHz}, \mathrm{CDCl}_{3}\right) \delta 136.9,132.9,131.7,128.9,123.1,119.5,109.5,109.2$, 108.1, 13.4; HRMS (ESI-TOF) calcd for $\mathrm{C}_{12} \mathrm{H}_{11} \mathrm{~N}_{2}{ }^{+}[\mathrm{M}+\mathrm{H}]^{+}$183.0917, found

183.0916. 
2-isobutyl-5-(thiophen-2-yl)-1H-pyrrole (4o):

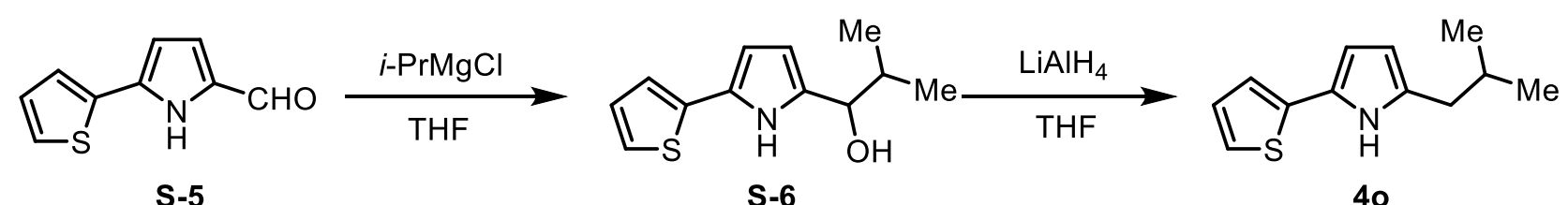

Step I- To a magnetically stirred solution of aldehyde S-5 $(240 \mathrm{mg}, 1.35 \mathrm{mmol})$ in THF $(5 \mathrm{~mL})$ at $0{ }^{\circ} \mathrm{C}$ was added isopropylmagnesium chloride solution ( $2 \mathrm{~mL} 1.3 \mathrm{M}$ solution in THF, $2.7 \mathrm{mmol}$ ). The reaction mixture was slowly warmed to $23^{\circ} \mathrm{C}$ and stirred for additional $1 \mathrm{~h}$. After completion of reaction, indicated by TLC the reaction mixture was cooled to $0{ }^{\circ} \mathrm{C}$ and quenched with saturated solution of $\mathrm{NH}_{4} \mathrm{Cl}$ and organic layer was extracted in EtOAc $(3 \times 10 \mathrm{~mL})$. Combined organic layers were washed with brine, dried over $\mathrm{Na} 2 \mathrm{SO} 4$ and concentrated in vacuo to afford crude alcohol S-6 as a pale-yellow oil which was used in next step without purification.

Step II- To a stirred solution of the so-obtained crude residue S-6 in THF $(5 \mathrm{~mL})$ at $0{ }^{\circ} \mathrm{C}$ was added $\mathrm{LiAlH}_{4}$ $(86 \mathrm{mg}, 2.26 \mathrm{mmol})$. After $5 \mathrm{~min}$, the reaction mixture was heated to reflux. After completion of reaction, indicated by TLC, reaction mixture cooled to $0{ }^{\circ} \mathrm{C}$ and quenched by slow addition of EtOAc $(2 \mathrm{~mL})$ and then water $(3 \mathrm{~mL})$, and allowed to warm to $25^{\circ} \mathrm{C}$. The two phases were separated, and the aqueous layer was extracted with EtOAc $(3 \times 10 \mathrm{~mL})$. The combined organic layers were dried over anhydrous $\mathrm{Na}_{2} \mathrm{SO}_{4}$, filtered, and concentrated under reduced pressure. The obtained residue was automated silica gel column chromatography to afford pure pyrrole 40 (43 mg, $194 \mu \mathrm{mol}, 14 \%$ yield for two steps) as pale-yellow oil.

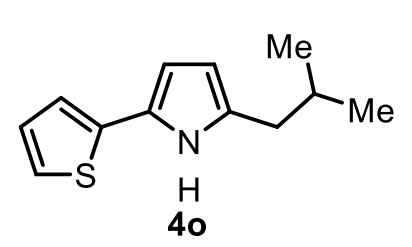

4o: $R_{\mathrm{f}}=0.6$ (silica gel, $20 \%$ EtOAc in hexanes); ${ }^{1} \mathbf{H}$ NMR $\left(400 \mathrm{MHz}, \mathrm{CDCl}_{3}\right) \delta$ 7.94 (br. s, 1H), $7.10(\mathrm{dd}, J=5.0,1.2 \mathrm{~Hz}, 1 \mathrm{H}), 7.03-6.91(\mathrm{~m}, 2 \mathrm{H}), 6.32(\mathrm{t}, J=$ $3.0 \mathrm{~Hz}, 1 \mathrm{H}), 5.99-5.83(\mathrm{~m}, 1 \mathrm{H}), 2.49(\mathrm{~d}, J=7.1 \mathrm{~Hz}, 2 \mathrm{H}), 1.88(\mathrm{dh}, J=13.7$, $6.8 \mathrm{~Hz}, 1 \mathrm{H}), 0.97(\mathrm{~d}, J=6.6 \mathrm{~Hz}, 6 \mathrm{H}) ;{ }^{13} \mathbf{C}\left\{{ }^{1} \mathbf{H}\right\} \mathbf{N M R}\left(101 \mathrm{MHz}, \mathrm{CDCl}_{3}\right) \delta$ 136.9, 133.0, 127.7, 125.2, 122.0, 120.1, 108.0, 107.0, 37.4, 29.4, 22.6; HRMS (ESI-TOF) calcd for $\mathrm{C}_{12} \mathrm{H}_{16} \mathrm{NS}^{+}[\mathrm{M}+\mathrm{H}]^{+}$206.0998, found 206.0990. 


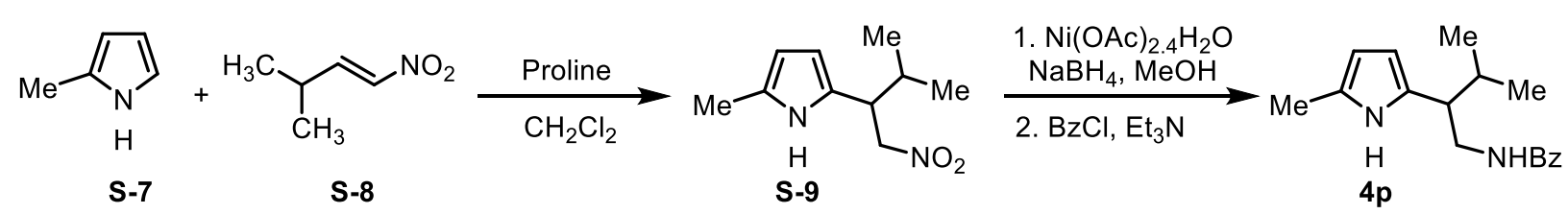

(rac)-2-methyl-5-(3-methyl-1-nitrobutan-2-yl)-1H-pyrrole (S-9): To a magnetically stirred solution of<smiles>Cc1ccc(C(C[N+](=O)[O-])C(C)C)[nH]1</smiles>

S-9 2-methyl pyrrole $\mathbf{S}-7$ (200 $\mathrm{mg}, 2.47 \mathrm{mmol})$ in $\mathrm{CH}_{2} \mathrm{Cl}_{2}(5 \mathrm{~mL})$ was added $(E)$-3methyl-1-nitrobut-1-ene $\mathbf{S - 8}$ (567 mg, $4.94 \mathrm{mmol}$ ) followed by ( \pm )-proline (56 mg, $0.49 \mathrm{mmol}$ ) at $23{ }^{\circ} \mathrm{C}$ and the reaction mixture stirred for $12 \mathrm{~h}$. After completion of reaction, indicated by TLC, reaction was quenched by sat. $\mathrm{NaHCO}_{3}(2 \mathrm{~mL})$ and water $(3 \mathrm{~mL})$. The two phases were separated, and the aqueous layer was extracted with $\mathrm{CH}_{2} \mathrm{Cl}_{2}(3 \times 10 \mathrm{~mL})$. The combined organic layers were dried over anhydrous $\mathrm{Na}_{2} \mathrm{SO}_{4}$, filtered, and concentrated under reduced pressure. The obtained residue was automated silica gel column chromatography to afford pyrrole $\mathbf{S - 9}\left(358 \mathrm{mg}, 1.82 \mathrm{mmol}, 74 \%\right.$ yield) as pale-yellow oil. $\mathbf{S - 9}: R_{\mathrm{f}}=0.35$ (silica gel, 20\% EtOAc in hexanes); ${ }^{1} \mathbf{H}$ NMR $\left(400 \mathrm{MHz}, \mathrm{CDCl}_{3}\right) \delta 7.66$ (br s, 1H), $5.82(\mathrm{t}, J=3.0 \mathrm{~Hz}, 1 \mathrm{H})$, $5.81-5.77(\mathrm{~m}, 1 \mathrm{H}), 4.64(\mathrm{dd}, J=12.6,6.1 \mathrm{~Hz}, 1 \mathrm{H}), 4.56(\mathrm{dd}, J=12.6,9.0 \mathrm{~Hz}, 1 \mathrm{H}), 3.28(\mathrm{dt}, J=9.0,6.3$ $\mathrm{Hz}, 1 \mathrm{H}), 2.22(\mathrm{~d}, J=0.9 \mathrm{~Hz}, 3 \mathrm{H}), 1.93(\mathrm{~h}, J=6.7 \mathrm{~Hz}, 1 \mathrm{H}), 0.96(\mathrm{~d}, J=6.8 \mathrm{~Hz}, 3 \mathrm{H}), 0.91(\mathrm{~d}, J=6.7 \mathrm{~Hz}$, $3 \mathrm{H}) ;{ }^{13} \mathbf{C}\left\{{ }^{1} \mathbf{H}\right\}$ NMR $\left(101 \mathrm{MHz}, \mathrm{CDCl}_{3}\right) \delta 127.2,127.1,106.4,106.4,78.5,44.2,30.8,20.7,19.6,13.1$; HRMS (ESI-TOF) calcd for $\mathrm{C}_{10} \mathrm{H}_{17} \mathrm{~N}_{2} \mathrm{O}_{2}{ }^{+}[\mathrm{M}+\mathrm{H}]^{+}$197.1285, found 197.1290.

\section{(rac)- $N$-(3-methyl-2-(5-methyl-1H-pyrrol-2-yl)butyl)benzamide (4p):}

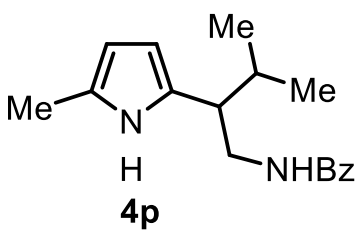

Step1: Pyrrole S-9 (200 mg, $1.02 \mathrm{mmol})$ was dissolved in methanol (5 mL), followed by the addition of $\mathrm{Ni}(\mathrm{OAc})_{2} \cdot 4 \mathrm{H}_{2} \mathrm{O}(254 \mathrm{mg}, 1.02 \mathrm{mmol})$ in one portion. Sodium borohydride ( $97 \mathrm{mg}, 2.55 \mathrm{mmol}$ ) was added to the solution in three portions over $15 \mathrm{~min}$ at $0{ }^{\circ} \mathrm{C}$. The reaction mixture turned black. After $2 \mathrm{~h}$, the starting material was consumed as based on TLC. The solvent was removed by rotary evaporation, and the residue was redissolved in $\mathrm{CH}_{2} \mathrm{Cl}_{2}(15 \mathrm{~mL})$ with the subsequent addition of concentrated ammonium hydroxide $(15 \mathrm{~mL})$. The mixture was stirred for $1.5 \mathrm{~h}$, and two layers were separated. The aqueous layer was extracted with $\mathrm{CH}_{2} \mathrm{Cl}_{2}(3 \times 15 \mathrm{~mL})$. The combined organic layers were dried with $\mathrm{MgSO}_{4}$. The filtration of the drying agent, followed by concentration via rotary evaporation, afforded a crude residue which was used directly for next step.

Step 2: To a magnetically stirred solution of so obtained crude amine in $\mathrm{CH}_{2} \mathrm{Cl}_{2}(5 \mathrm{~mL})$ was added triethyl amine $(212 \mu \mathrm{L}, 1.53 \mathrm{mmol})$ followed by dropwise addition of benzoyl chloride $(124 \mu \mathrm{L}, 1.07 \mathrm{mmol})$ at 0 ${ }^{\circ} \mathrm{C}$ and the reaction mixture stirred for additional $2 \mathrm{~h}$ at same temperature. After completion of reaction, indicated by TLC, reaction was quenched by sat. $\mathrm{NaHCO}_{3}(2 \mathrm{~mL})$ and water $(3 \mathrm{~mL})$. The two phases were 
separated, and the aqueous layer was extracted with $\mathrm{CH}_{2} \mathrm{Cl}_{2}(3 \times 10 \mathrm{~mL})$. The combined organic layers were dried over anhydrous $\mathrm{Na}_{2} \mathrm{SO}_{4}$, filtered, and concentrated under reduced pressure. The obtained residue was automated silica gel column chromatography to afford pyrrole $4 \mathbf{p}$ (174 mg, $0.64 \mathrm{mmol}, 63 \%$ yield) as paleyellow viscous liquid. $4 \mathbf{p}: R_{\mathrm{f}}=0.45$ (silica gel, $30 \%$ EtOAc in hexanes); ${ }^{1} \mathbf{H}$ NMR $\left(400 \mathrm{MHz}, \mathrm{CDCl}_{3}\right) \delta 7.99$ (br. s, 1H), $7.64-7.56$ (m, 2H), $7.50-7.42$ (m, 1H), 7.37 (dd, J = 8.2, $6.7 \mathrm{~Hz}, 2 \mathrm{H}), 6.15$ (br. s, 1H), 5.86 $(\mathrm{t}, J=3.0 \mathrm{~Hz}, 1 \mathrm{H}), 5.83-5.77(\mathrm{~m}, 1 \mathrm{H}), 3.95(\mathrm{ddd}, J=13.2,7.0,4.9 \mathrm{~Hz}, 1 \mathrm{H}), 3.35(\mathrm{ddd}, J=13.2,10.6,4.3$ $\mathrm{Hz}, 1 \mathrm{H}), 2.65(\mathrm{ddd}, J=10.6,7.2,4.9 \mathrm{~Hz}, 1 \mathrm{H}), 2.23$ (d, $J=0.9 \mathrm{~Hz}, 3 \mathrm{H}), 1.87$ (h, $J=6.8 \mathrm{~Hz}, 1 \mathrm{H}), 1.00$ (d, $J$ $=6.7 \mathrm{~Hz}, 3 \mathrm{H}), 0.88(\mathrm{~d}, J=6.7 \mathrm{~Hz}, 3 \mathrm{H}) ;{ }^{13} \mathbf{C}\left\{{ }^{1} \mathbf{H}\right\} \mathbf{N M R}\left(101 \mathrm{MHz}, \mathrm{CDCl}_{3}\right) \delta 167.7,134.8,131.5,130.2$, 128.6, 127.0, 126.9, 106.4, 105.9, 46.1, 42.2, 31.6, 20.9, 20.5, 13.2; HRMS (ESI-TOF) calcd for $\mathrm{C}_{17} \mathrm{H}_{23} \mathrm{~N}_{2} \mathrm{O}^{+}[\mathrm{M}+\mathrm{H}]^{+}$271.1805, found 271.1811.

(rac)-tert-butyl benzoyl(3-methyl-2-(5-methyl-1H-pyrrol-2-yl)butyl)carbamate (4q):

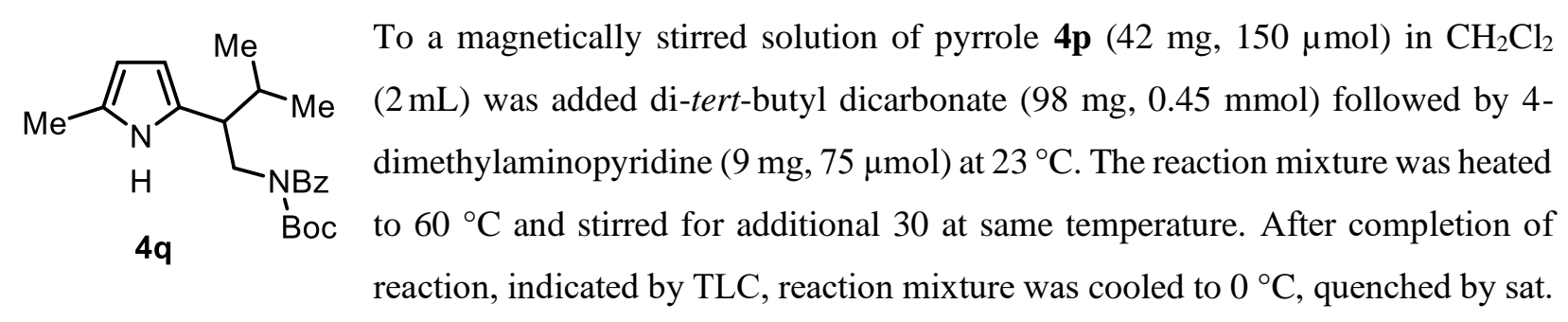
$\mathrm{NaHCO}_{3}(2 \mathrm{~mL})$ and water $(3 \mathrm{~mL})$. The two phases were separated, and the aqueous layer was extracted with EtOAc $(3 \times 5 \mathrm{~mL})$. The combined organic layers were dried over anhydrous $\mathrm{Na}_{2} \mathrm{SO}_{4}$, filtered, and concentrated under reduced pressure. The obtained residue was automated silica gel column chromatography to afford pyrrole $\mathbf{4 q}\left(51 \mathrm{mg}, 137 \mu \mathrm{mol}, 89 \%\right.$ yield) as yellow solid. $\mathbf{4 q}: R_{\mathrm{f}}=0.35$ (silica gel, 20\% EtOAc in hexanes); ${ }^{1} \mathbf{H}$ NMR (400 MHz, $\left.\mathrm{CDCl}_{3}\right) \delta 7.95$ (br. s, 1H), $7.42-7.33(\mathrm{~m}, 1 \mathrm{H}), 7.25(\mathrm{t}$, $J=7.8 \mathrm{~Hz}, 2 \mathrm{H}), 7.12-6.93(\mathrm{~m}, 2 \mathrm{H}), 5.75(\mathrm{t}, J=2.9 \mathrm{~Hz}, 1 \mathrm{H}), 5.72(\mathrm{dt}, J=3.9,2.0 \mathrm{~Hz}, 1 \mathrm{H}), 4.20(\mathrm{dd}, J=$ $13.7,11.5 \mathrm{~Hz}, 1 \mathrm{H}), 3.98(\mathrm{dd}, J=13.7,6.2 \mathrm{~Hz}, 1 \mathrm{H}), 3.11(\mathrm{dt}, J=11.9,6.2 \mathrm{~Hz}, 1 \mathrm{H}), 2.16(\mathrm{~d}, J=0.9 \mathrm{~Hz}, 3 \mathrm{H})$, $1.88-1.75(\mathrm{~m}, 1 \mathrm{H}), 1.03(\mathrm{~s}, 9 \mathrm{H}), 1.00(\mathrm{~d}, J=6.8 \mathrm{~Hz}, 3 \mathrm{H}), 0.87(\mathrm{~d}, J=6.7 \mathrm{~Hz}, 3 \mathrm{H}) ;{ }^{13} \mathbf{C}\left\{{ }^{1} \mathbf{H}\right\} \mathbf{N M R}(101$ $\left.\mathrm{MHz}, \mathrm{CDCl}_{3}\right) \delta 174.0,154.0,138.4,130.8,129.0,127.9,127.5,126.3,108.4,105.6,83.0,47.1,43.9,31.9$, 27.4, 21.2, 20.0, 13.1; HRMS (ESI-TOF) calcd for $\mathrm{C}^{22} \mathrm{H}^{31} \mathrm{~N}^{2} \mathrm{O}^{3+}[\mathrm{M}+\mathrm{H}]^{+} 371.2329$, found 371.2333. 


\section{Limitations}

Fig. S1. Low yielding substrates with no 2-position substitution on indoles/pyrroles.

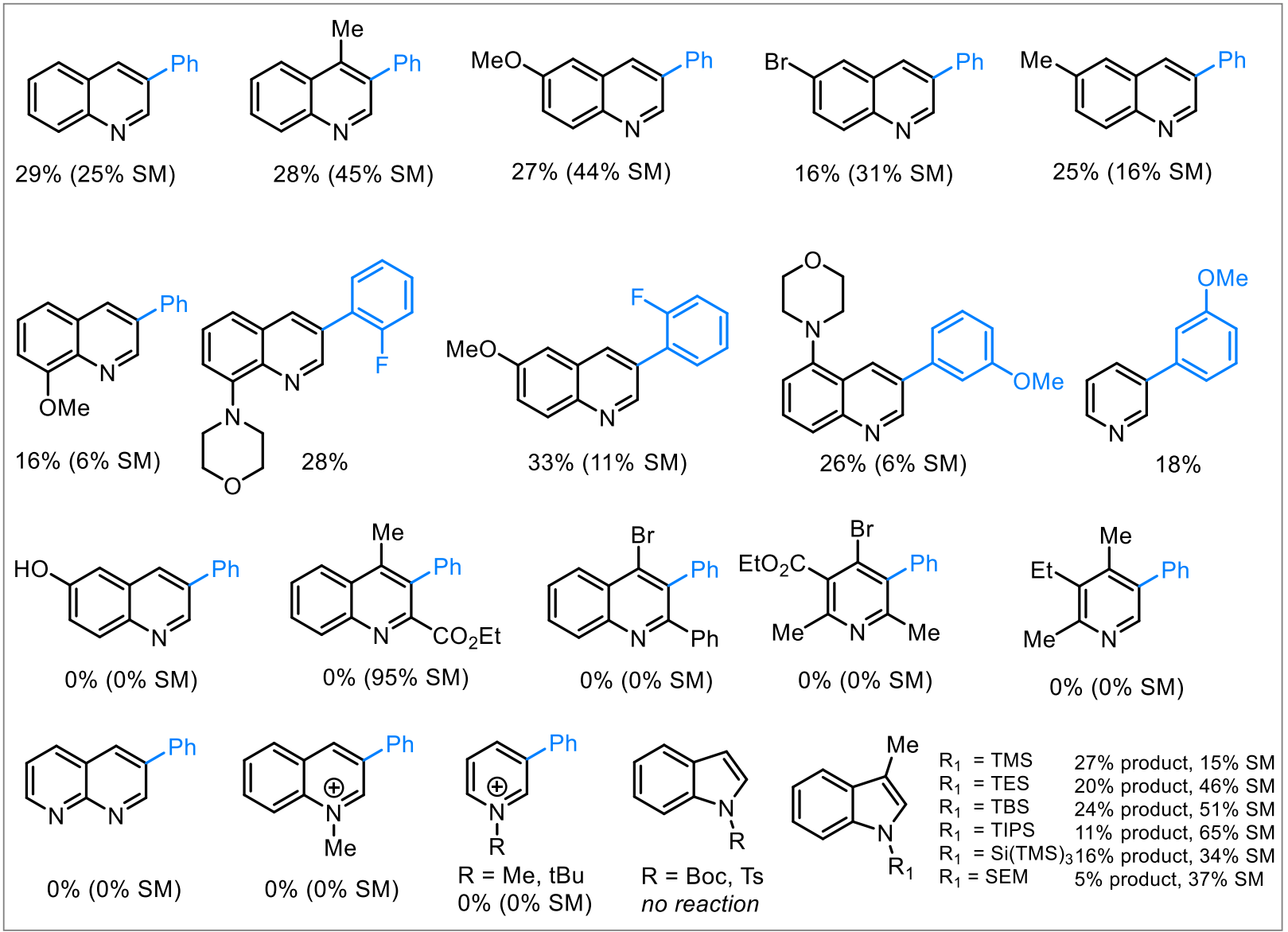

Fig. S2. Limitations of diazirines (A) electron rich aromatic halo diazirine were converted to corresponding aldehyde. (B) Aliphatic diazirines bearing $\alpha$-hydrogens were isomerized to chloro-olefins (C) Aliphatic tert-alkyl diazirines underwent competitive dimerization.
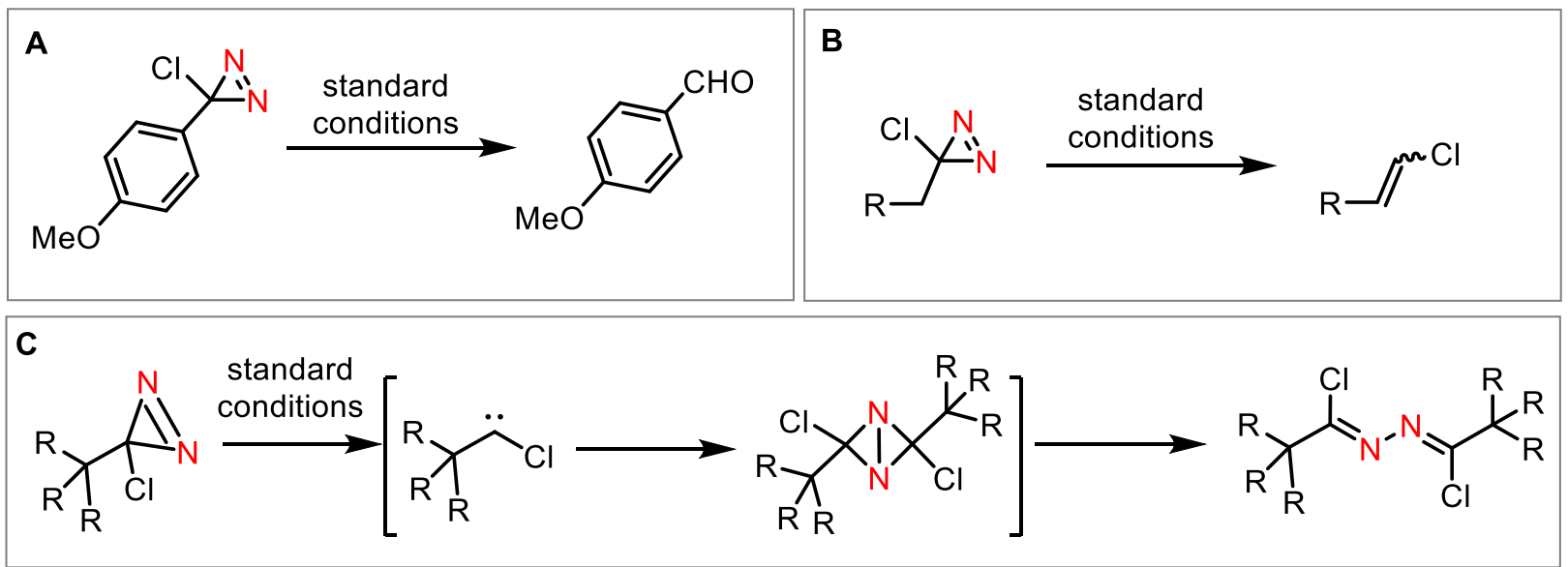


\section{Comparison with Ciamician-Denstedt Reaction}

i) Under standard conditions:

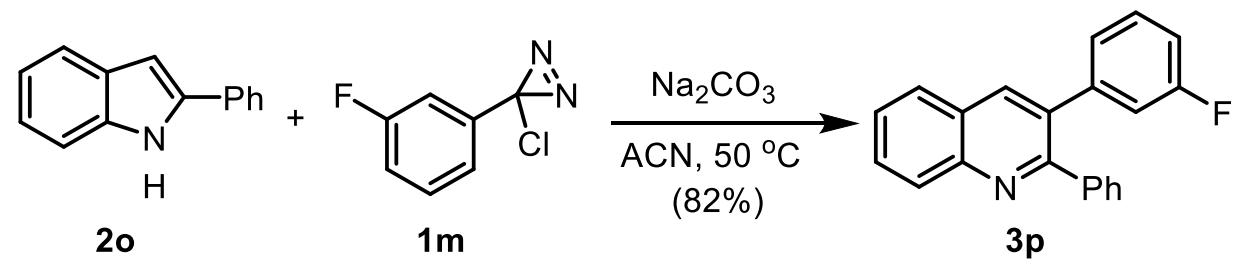

Following the general procedure II, product 3p was isolated in $82 \%$ yield as a yellow solid from 2-phenyl indole $2 \mathrm{o}$ and diazirine 1m. 3p: ${ }^{1} \mathbf{H}$ NMR $\left(400 \mathrm{MHz}, \mathrm{CDCl}_{3}\right) \delta 8.21(\mathrm{~d}, J=8.5 \mathrm{~Hz}, 1 \mathrm{H}), 8.17(\mathrm{~s}, 1 \mathrm{H}), 7.88$ $(\mathrm{dd}, J=8.2,1.4 \mathrm{~Hz}, 1 \mathrm{H}), 7.76(\mathrm{ddd}, J=8.4,6.8,1.4 \mathrm{~Hz}, 1 \mathrm{H}), 7.58(\mathrm{td}, J=7.5,6.9,1.1 \mathrm{~Hz}, 1 \mathrm{H}), 7.49-7.40$ (m, 2H), $7.30(\mathrm{dd}, J=5.2,1.9 \mathrm{~Hz}, 3 \mathrm{H}), 7.26-7.20(\mathrm{~m}, 1 \mathrm{H}), 7.04-6.87(\mathrm{~m}, 3 \mathrm{H})$.

\section{ii) Under Ciamician-Denstedt followed by Suzuki coupling conditions:}

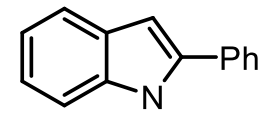

$20^{\mathrm{H}}$

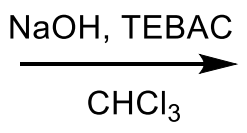

$(45 \%)$

Overall yield: $22 \%$

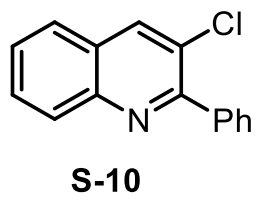

S-10

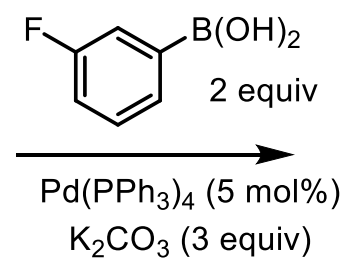

toluene: $\mathrm{H}_{2} \mathrm{O}(9: 1)$

$100^{\circ} \mathrm{C}, 30 \mathrm{~h}$

$(48 \%)$<smiles>Fc1cccc(-c2cc3ccccc3nc2-c2ccccc2)c1</smiles><smiles>Clc1cc2ccccc2nc1-c1ccccc1</smiles>

Step I- The reaction was performed according to reported literature procedure. ${ }^{20} \mathrm{NaOH}$ $(104 \mathrm{mg}, 2.6 \mathrm{mmol})$ in water $(0.5 \mathrm{~mL})$ was added to a vigorously stirred solution of 2phynylindole $2 \mathrm{o}(50 \mathrm{mg}, 0.26 \mathrm{mmol}, 1 \mathrm{eq})$ and benzyltriethylammonium chloride (10 $\mathrm{mg}, 26 \mu \mathrm{mol}, 10 \mathrm{~mol} \%$ ) in chloroform ( $3 \mathrm{~mL}$ ) under ice-cooling bath. The reaction mixture was stirred at $0{ }^{\circ} \mathrm{C}$ for $3 \mathrm{~h}$ and left overnight at room temperature. The aqueous layer was separated and extracted with chloroform $(3 \times 5 \mathrm{~mL})$. The organic layers were dried over $\mathrm{MgSO}_{4}$. After filtration and evaporation, the crude was purified by flash chromatography (10\% EtOAc/Hexanes) affording 3-chloro2-phenyl quinoline $\mathbf{S - 1 0}$ as a brown oil (28 $\mathrm{mg}, 116 \mu \mathrm{mol}, 45 \%)$ and $18 \%$ of starting material 20 was recovered. $R_{\mathrm{f}}=0.61\left(20 \%\right.$ Ethyl acetate/Hexanes). ${ }^{1} \mathbf{H} \mathbf{N M R}\left(400 \mathrm{MHz}, \mathrm{CDCl}_{3}\right) \delta 8.19(\mathrm{~d}, J=0.8 \mathrm{~Hz}, 1 \mathrm{H})$, $8.06(\mathrm{dq}, J=8.5,0.9 \mathrm{~Hz}, 1 \mathrm{H}), 7.74-7.67(\mathrm{~m}, 3 \mathrm{H}), 7.64(\mathrm{ddd}, J=8.5,6.9,1.5 \mathrm{~Hz}, 1 \mathrm{H}), 7.49$ (ddd, $J=8.1$, $6.9,1.2 \mathrm{~Hz}, 1 \mathrm{H}), 7.43-7.38(\mathrm{~m}, 3 \mathrm{H})$. Spectroscopic data are in agreement with those in the literature. ${ }^{21}$ 


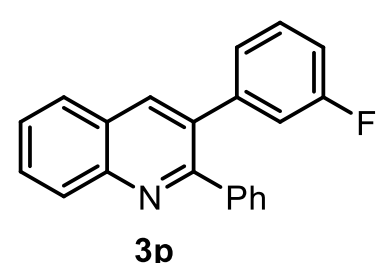

$3 p$

Step II- To an oven dried 1 dram screw cap vial equipped with a stir bar and PTFE/white silicone septum was added quinoline $\mathbf{S - 1 0}$ (28 mg, $0.116 \mathrm{mmol}), 3$ fluoroboronic acid $(32.6 \mathrm{mg}, 0.23 \mathrm{mmol}), \mathrm{Pd}\left(\mathrm{PPh}_{3}\right)_{4}(6.7 \mathrm{mg}, 5.8 \mu \mathrm{mol})$ and $\mathrm{K}_{2} \mathrm{CO}_{3}$ (48 mg, $0.348 \mathrm{mmol}$ ). The vial was evacuated/backfilled with nitrogen twice, and then toluene $(0.9 \mathrm{~mL})$ and water $(0.1 \mathrm{~mL})$ (sparged with nitrogen for $15 \mathrm{~min}$ ) were added via syringe. The vial was stirred vigorously $(1000 \mathrm{rpm})$ at $100{ }^{\circ} \mathrm{C}$. After $30 \mathrm{~h}$, vial was cooled to room temperature, reaction was quenched by sat. $\mathrm{NH}_{4} \mathrm{Cl}(2 \mathrm{~mL})$ and water $(2 \mathrm{~mL})$. The two phases were separated, and the aqueous layer was extracted with EtOAc $(3 \times 3 \mathrm{~mL})$. The combined organic layers were dried over anhydrous $\mathrm{Na}_{2} \mathrm{SO}_{4}$, filtered, and concentrated under reduced pressure. The obtained residue was automated silica gel column chromatography to afford quinoline $\mathbf{3 p}$ (16.8 $\mathrm{mg}, 56 \mu \mathrm{mol}, 48 \%$ yield) as yellow solid, while $21 \%$ of starting material $(6 \mathrm{mg}, 25 \mu \mathrm{mol})$ was recovered back. 


\section{Mechanistic Experiments}

\section{VIA. Effect of tetrabutyl ammonium chloride}

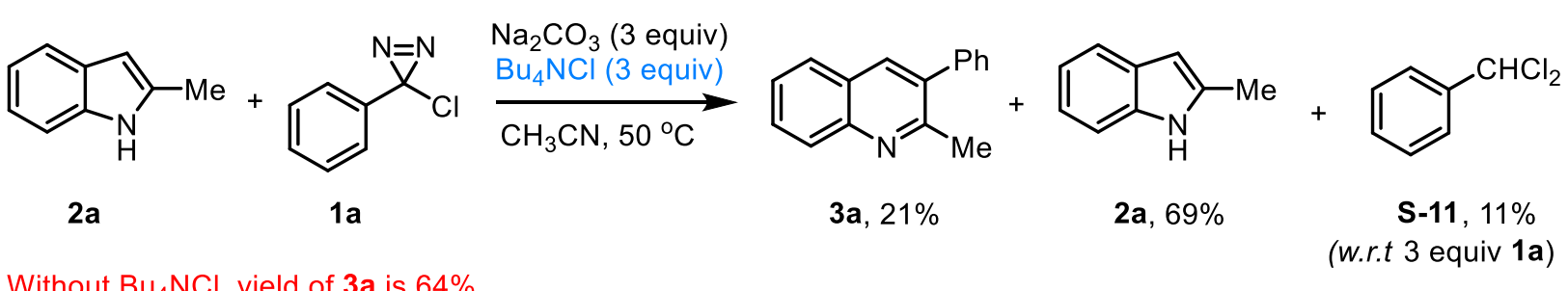

Without $\mathrm{Bu}_{4} \mathrm{NCl}$, yield of $3 \mathrm{a}$ is $64 \%$

Oven dried 1-dram screw capped vial was degassed and charged with 2-methylindole 2a (10.0 mg, 76.2 $\mu$ mol, 1.0 equiv), diazirine 1a (35.0 mg, $229 \mu \mathrm{mol}, 3.0$ equiv), $\mathrm{Na}_{2} \mathrm{CO}_{3}(24.0 \mathrm{mg}, 229 \mu \mathrm{mol}, 3.0$ equiv), $\mathrm{Bu}_{4} \mathrm{NCl}(63.0 \mathrm{mg}, 229 \mu \mathrm{mol}, 3.0$ equiv) and $1 \mathrm{~mL}$ of dry acetonitrile. This mixture was then allowed to stir at $50{ }^{\circ} \mathrm{C}$ for $12 \mathrm{~h}$ and then allowed to cool to $25^{\circ} \mathrm{C}$. The reaction mixture was quenched by the addition of saturated aqueous $\mathrm{NH}_{4} \mathrm{Cl}$ solution $(1 \mathrm{~mL})$, and the two phases were separated. The aqueous layer was extracted with EtOAc $(3 \times 3 \mathrm{~mL})$, and the combined organic layers were dried over anhydrous $\mathrm{Na}_{2} \mathrm{SO}_{4}$, filtered, and concentrated under reduced pressure. Crude NMR showed the presence of quinoline 3a (21\%), starting material 2a (69\%) and benzal chloride S-11 $11 \%$ (w.r.t. 3 equiv. of diazirine 1a).

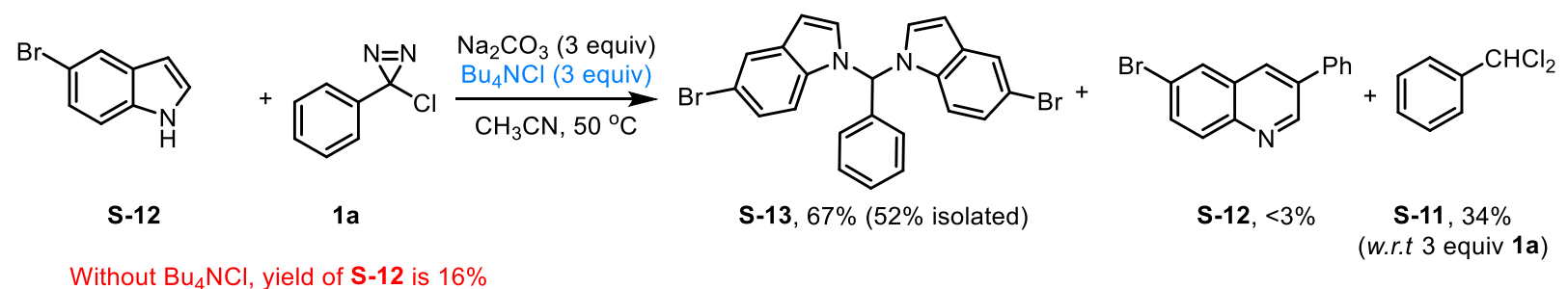

Oven dried 1-dram screw capped vial was degassed and charged with 5-bromoindole S-11 (10.0 mg, 47 $\mu \mathrm{mol}, 1.0$ equiv), diazirine $1 \mathbf{a}\left(22.0 \mathrm{mg}, 143 \mu \mathrm{mol}, 3.0\right.$ equiv), $\mathrm{Na}_{2} \mathrm{CO}_{3}(15.0 \mathrm{mg}, 143 \mu \mathrm{mol}, 3.0$ equiv), $\mathrm{Bu}_{4} \mathrm{NCl}(39.7 \mathrm{mg}, 143 \mu \mathrm{mol}, 3.0$ equiv) and $1 \mathrm{~mL}$ of dry acetonitrile. This mixture was then allowed to stir at $50{ }^{\circ} \mathrm{C}$ for $12 \mathrm{~h}$ and then allowed to cool to $25^{\circ} \mathrm{C}$. The reaction mixture was quenched by the addition of saturated aqueous $\mathrm{NH}_{4} \mathrm{Cl}$ solution $(1 \mathrm{~mL})$, and the two phases were separated. The aqueous layer was extracted with EtOAc $(3 \times 3 \mathrm{~mL})$, and the combined organic layers were dried over anhydrous $\mathrm{Na}_{2} \mathrm{SO}_{4}$, filtered, and concentrated under reduced pressure. Crude NMR showed the presence of S-13 (67\%), quinoline S-12 (<3\%), and benzal chloride S-11, 34\% (w.r.t. 3 equiv. of diazirine 1a). Purification of crude material on automated column chromatography afforded $\mathbf{S - 1 3}(6.0 \mathrm{mg}, 13 \mu \mathrm{mol}, 52 \%)$ as a yellow viscous liquid. 


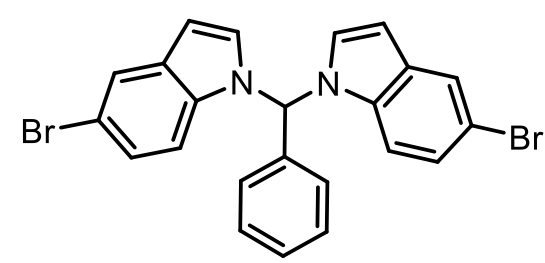

S-13

1,1'-(phenylmethylene)bis(5-bromo-1H-indole) (S-13) BD1-153): ${ }^{1}$ H NMR $\left(400 \mathrm{MHz}, \mathrm{CDCl}_{3}\right) \delta 7.84(\mathrm{~s}, 1 \mathrm{H}), 7.78(\mathrm{~d}, J=1.9 \mathrm{~Hz}, 2 \mathrm{H})$, $7.44-7.37(\mathrm{~m}, 3 \mathrm{H}), 7.24(\mathrm{dd}, J=8.7,1.9 \mathrm{~Hz}, 2 \mathrm{H}), 7.11-7.01(\mathrm{~m}$, 4H), $6.73(\mathrm{~d}, J=3.4 \mathrm{~Hz}, 2 \mathrm{H}), 6.47(\mathrm{dd}, J=3.4,0.8 \mathrm{~Hz}, 2 \mathrm{H}) ;{ }^{13} \mathbf{C}\left\{{ }^{1} \mathbf{H}\right\}$ NMR $\left(101 \mathrm{MHz}, \mathrm{CDCl}_{3}\right) \delta 135.7,134.7,131.0,129.9,129.5,127.3$, 126.8, 125.5, 124.0, 114.1, 111.1, 102.9, 69.6; HRMS (ESI-TOF) calcd for $\mathrm{C}_{15} \mathrm{H}_{11} \mathrm{BrN}^{+}\left[\mathrm{M}-\mathrm{C}_{8} \mathrm{H}_{5} \mathrm{BrN}\right]^{+} 284.0069$, found 284.0069.

\section{VIB. Effect of addition of quinoline}

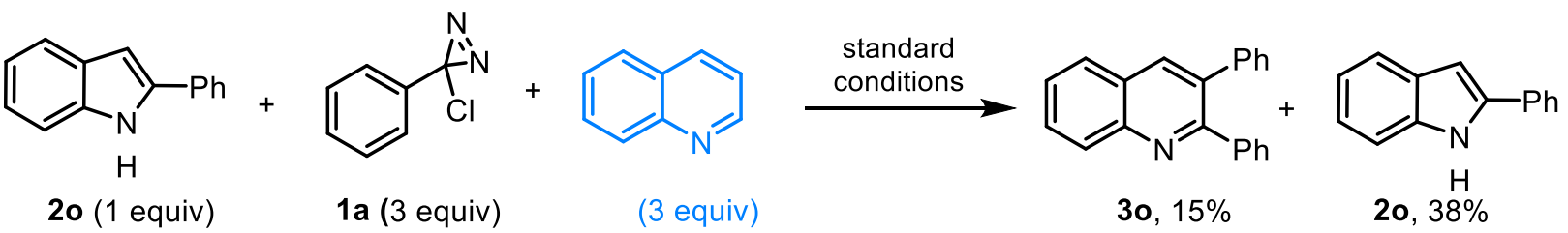

Without quinoline, yield of 30 is $68 \%$

Oven dried 1-dram screw capped vial was degassed and charged with 2-phenylindole 2a (10.0 mg, $52 \mu \mathrm{mol}$, 1.0 equiv), diazirine $1 \mathbf{a}\left(24 \mathrm{mg}, 155 \mu \mathrm{mol}, 3.0\right.$ equiv), $\mathrm{Na}_{2} \mathrm{CO}_{3}$ (16 mg, $155 \mu \mathrm{mol}, 3.0$ equiv), quinoline (20.0 mg, $155 \mu \mathrm{mol}, 3.0$ equiv) and $1 \mathrm{~mL}$ of dry acetonitrile. This mixture was then allowed to stir at $50{ }^{\circ} \mathrm{C}$ for $12 \mathrm{~h}$ and then allowed to cool to $25^{\circ} \mathrm{C}$. The reaction mixture was quenched by the addition of saturated aqueous $\mathrm{NH}_{4} \mathrm{Cl}$ solution $(1 \mathrm{~mL})$, and the two phases were separated. The aqueous layer was extracted with EtOAc $(3 \times 3 \mathrm{~mL})$, and the combined organic layers were dried over anhydrous $\mathrm{Na}_{2} \mathrm{SO}_{4}$, filtered, and concentrated under reduced pressure. Crude NMR showed the presence of 2,3-diphenylquinoline 3o (15\%) and starting material $20(38 \%)$. 


\section{X-Ray-derived ORTEP of 3z}
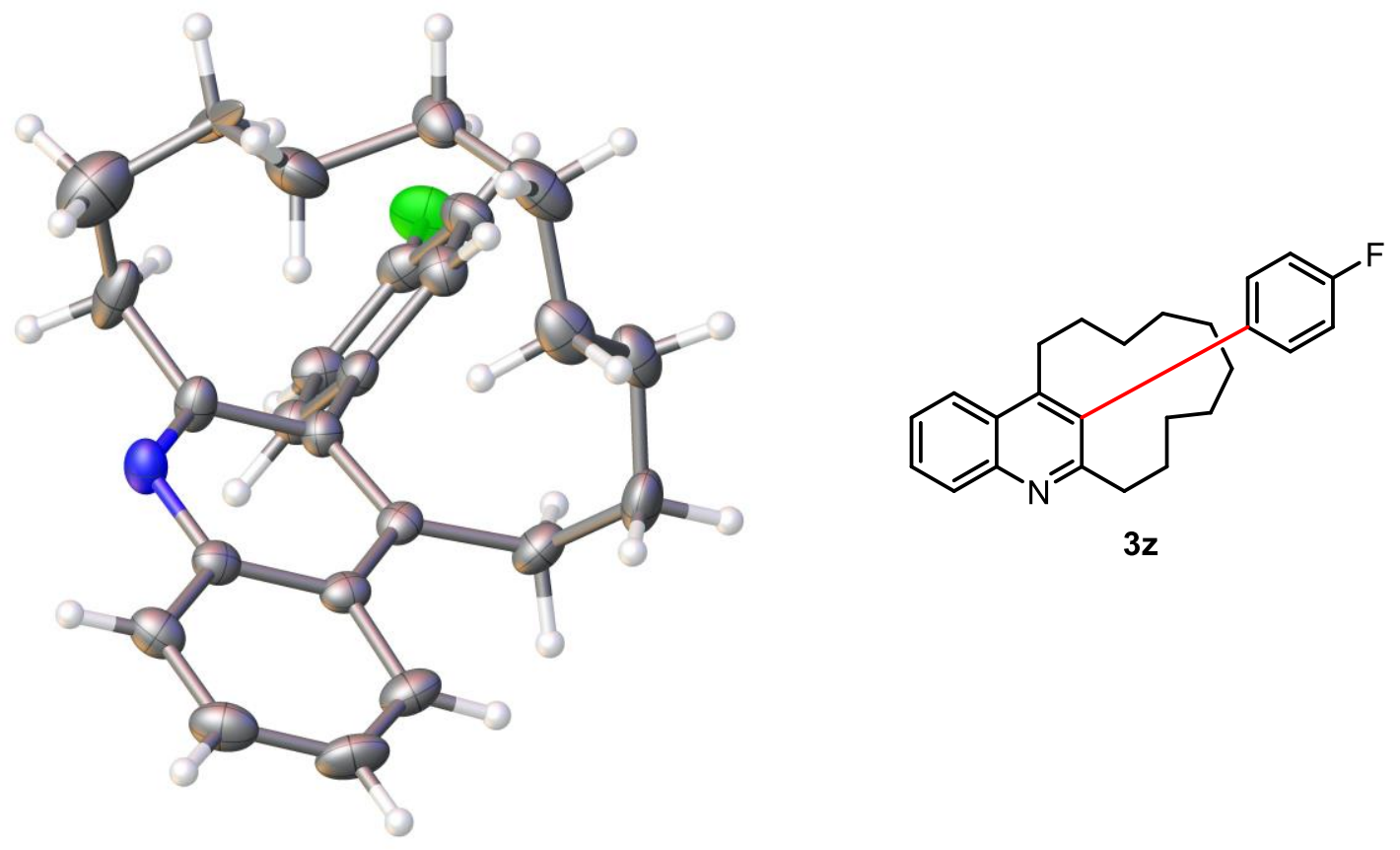

\section{ORTEP drawing of $3 z$}

Cystallographic data is available free of charge from the Cambridge Crystallographic Data Centre (https://summary.ccdc.cam.ac.uk/structure-summary-form under deposition number xxxxx). 


\section{Datablock: mo_0896_levin_0m}

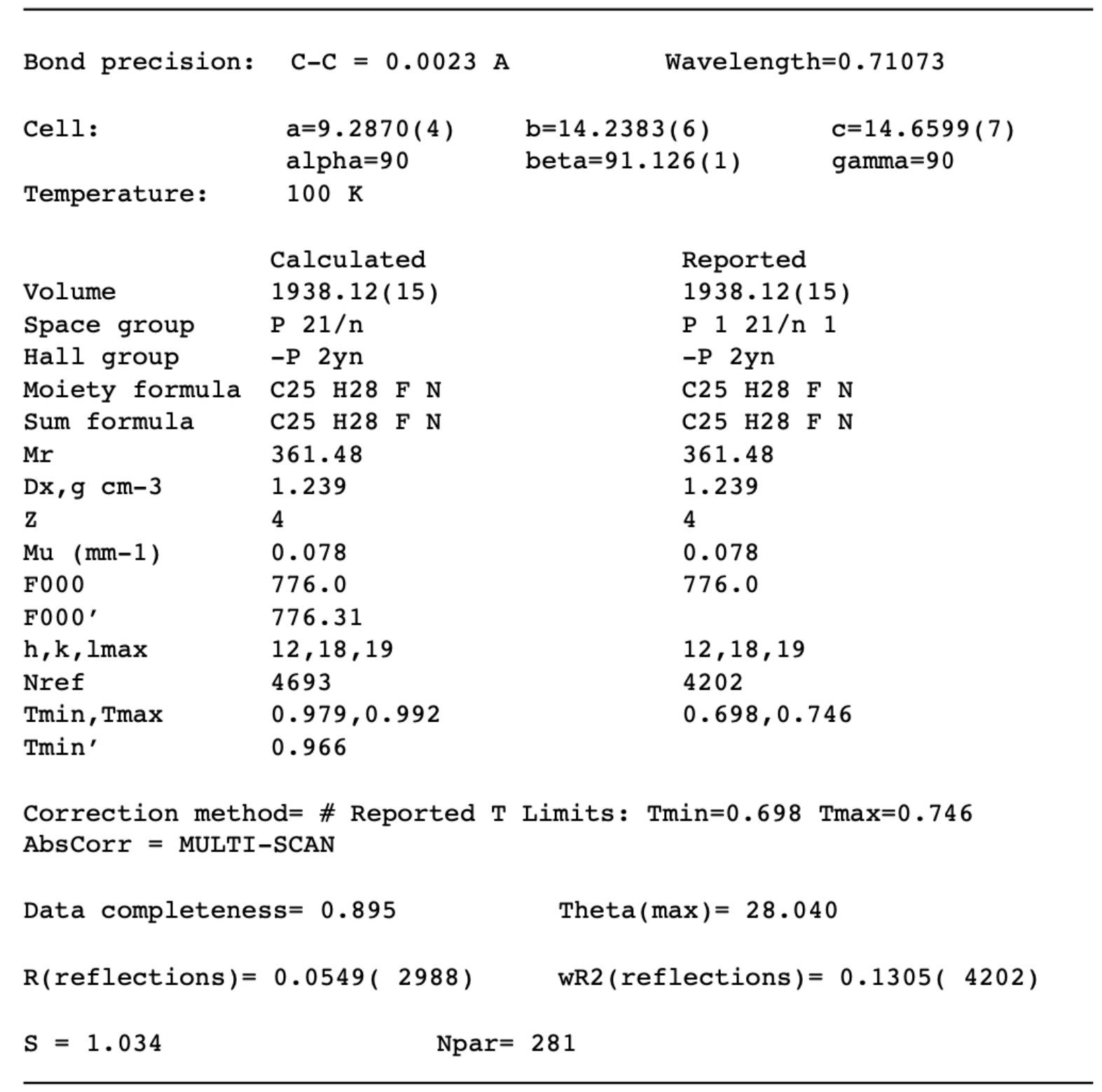




\section{References:}

1. Majumder, S.; Gipson, K. R.; Odom A. L. A Multicomponent Coupling Sequence for Direct Access to Substituted Quinolines Org. Lett. 2009, 11, 4720-4723 (3a)

2. Tseng, C.-H.; Lin, C.-K.; Chen, Y.-L.; Hsu, C.-Y. Wu, H.-N.; Tseng, C.-K.; Lee, J.-C. Synthesis, antiproliferative and anti-dengue virus evaluations of 2-aroyl-3-arylquinoline derivatives Eur. J. Med. Chem. 2014, 79, 66-76. (3b: )

3. McDaniel, T. J.; Lansdell, T. A.; Dissanayake, A. A.; Azevedo, L. M.; Claes, J.; Odom, A. L.; Tepe, J. J. Substituted quinolines as noncovalent proteasome inhibitor Bioorg. Med. Chem. 2016, 24, 2441-2450. (3j)

4. Kong, L.; Yu, S.; Zhou, X.; Li, X. Redox-Neutral Couplings between Amides and Alkynes via Cobalt(III)-Catalyzed C-H Activation Org. Lett. 2016, 18, 588-591. (31:)

5. Saunthwal, R. K.; Patel, M.; Verma, A. K. Metal- and Protection-Free [4 + 2] Cycloadditions of Alkynes with Azadienes: Assembly of Functionalized Quinolines Org. Lett. 2016, 18, 2200-2203. (30:)

6. Wei, Y.; Yoshikai, N. Modular Pyridine Synthesis from Oximes and Enals through Synergistic Copper/Iminium Catalysis J. Am. Chem. Soc. 2013, 135, 3756-3759. ( 5b:)

7. Wang, K.; Meng, L.-G.; Wang, L. Visible-Light-Promoted [2+2+2] Cyclization of Alkynes with Nitriles to Pyridines Using Pyrylium Salts as Photoredox Catalysts Org. Lett. 2017, 19, 1958-1961. (5f: Xx:)

8. Parthasarathy, K.; Jeganmohan, M.; Cheng, C.-H. Rhodium-Catalyzed One-Pot Synthesis of Substituted Pyridine Derivatives from $\alpha$, $\beta$-Unsaturated Ketoximes and Alkynes Org. Lett. 2008, 10, 325-328. (5f': $\mathbf{X x}:)$

9. Zhu, T.; Li, X.; Chang, H.; Gao, W.; Wei, W. Nano-Pd/Al(OH) ${ }_{3}$-Catalyzed Suzuki-Miyaura Coupling Reaction of Potassium Aryl- and Heteroaryltrifluoroborates with Electrophiles in Alcohols Synlett 2016, 27, 880-887. (51:)

10. Craig, D.; Paina, F.; Smith, S. C. Double decarboxylative Claisen rearrangement reactions: microwaveassisted de novo synthesis of pyridines Chem. Commun. 2008, 3408-3410. (5l': Xx':)

11. Wang, K.; Meng, L.-G. Wang, L. Visible-Light-Promoted [2+2+2] Cyclization of Alkynes with Nitriles to Pyridines Using Pyrylium Salts as Photoredox Catalysts Org. Lett. 2017, 19, 1958-1961. (5n:) 12. Huang, Z.; Zhang, W.-X.; Xi, Z. Lewis Acid-Promoted Ring-Contraction of 2,4,6,8-Tetrasubstituted 1,5-Diazacyclooctatetraenes to 2,4,6-Trisubstituted Pyridines Org. Lett. 2018, 20, 485-488. (5t': Xx':)

13. Ohashi, M.; Takeda, I.; Ikawa, M.; Ogoshi, S. Nickel-Catalyzed Dehydrogenative [4 + 2] Cycloaddition of 1,3-Dienes with Nitriles J. Am. Chem. Soc. 2011, 133, 18018-18021. (5v') 
14. Błachut, D.; Czarnocki, Z.; Wojtasiewicz, K. A Suzuki-Miyaura Approach to a Series of Forensically Relevant Pyridines Synthesis 2006, 17, 2855-2864. (5x)

15. a) Graham, W. H. The Halogenation of Amidines. I. Synthesis of 3-Halo- and Other Negatively Substituted Diazirines J. Am. Chem. Soc. 1965, 87, 4396-4397. b) Moss, R. A.; Tian, J. Hammett studies of aryldichloromethide carbanion reactions Org. Lett. 2006, 8, 1245-1247. (general procedure, 1a, 1c, 11)

16. Baird, M. S.; Bruce, I. Generation and trapping of 2- and 3-pyridylhalocarbenes from diazirines $J$. Chem. Research (S) 1989, 12, 374-375. (1b)

17. Martinu, T.; Dailey, W. P. Reactivity of 1-Chloro-3-phenyldiazirines J. Org. Chem. 2006, 71, 50125015. (1n)

18. Krasniqi, B.; Geerts, K.; Dehaen, W. General Transition Metal-Free Synthesis of NH-Pyrroles from Secondary Alcohols and 2-Aminoalcohols J. Org. Chem. 2019, 84, 5027-5034. (4e:)

19. Wang, H.-Y.; Mueller, D. S.; Sachwani, R. M.; Kapadia, R.; Londino, H. N.; Anderson, L. L. Regioselective Synthesis of 2,3,4- or 2,3,5-Trisubstituted Pyrroles via [3,3] or [1,3] Rearrangements of OVinyl Oximes J. Org. Chem. 2011, 76, 3203-3221. (4h:)

20. The reaction was performed using literature reported procedure as follow: Rousseaux, S.; Davi, M.; Sofack-Kreutzer, J.; Pierre, C.; Kefalidis, C. E.; Clot, E.; Fagnou, K.; Baudoin O. J. Am. Chem. Soc. 2010, 132, 10706-10716.

21. Akhil Mahajan, Anju Arya, and Tejpal Singh Chundawat Green synthesis of silver nanoparticles using green alga (Chlorella vulgaris) and its application for synthesis of quinolines derivatives Synth. Commun. 2019, 49, 1926-1937. 
VII. ${ }^{1} \mathrm{H},{ }^{13} \mathrm{C},{ }^{19} \mathrm{~F}$ and $2 \mathrm{D}$ NMR Spectra
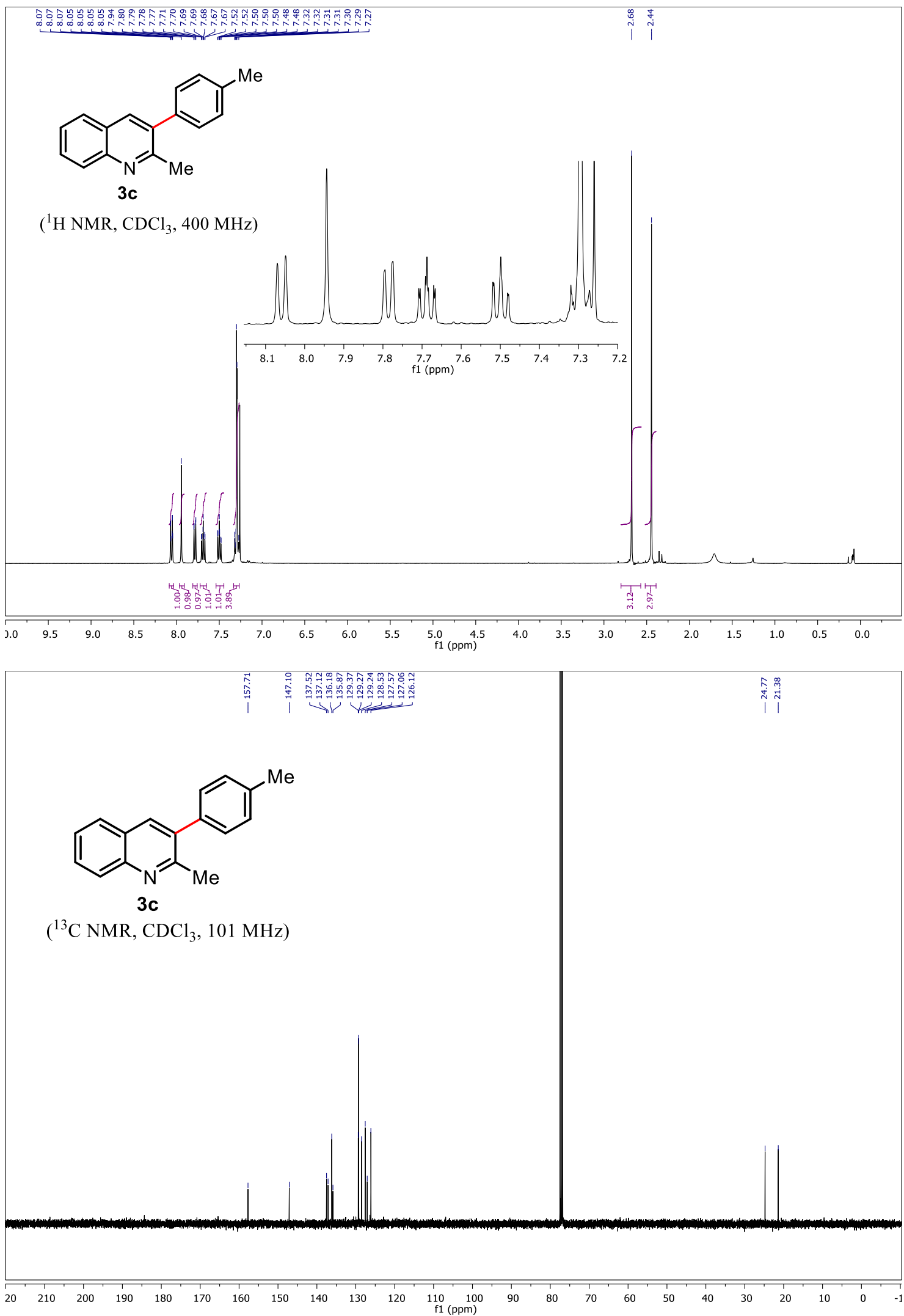

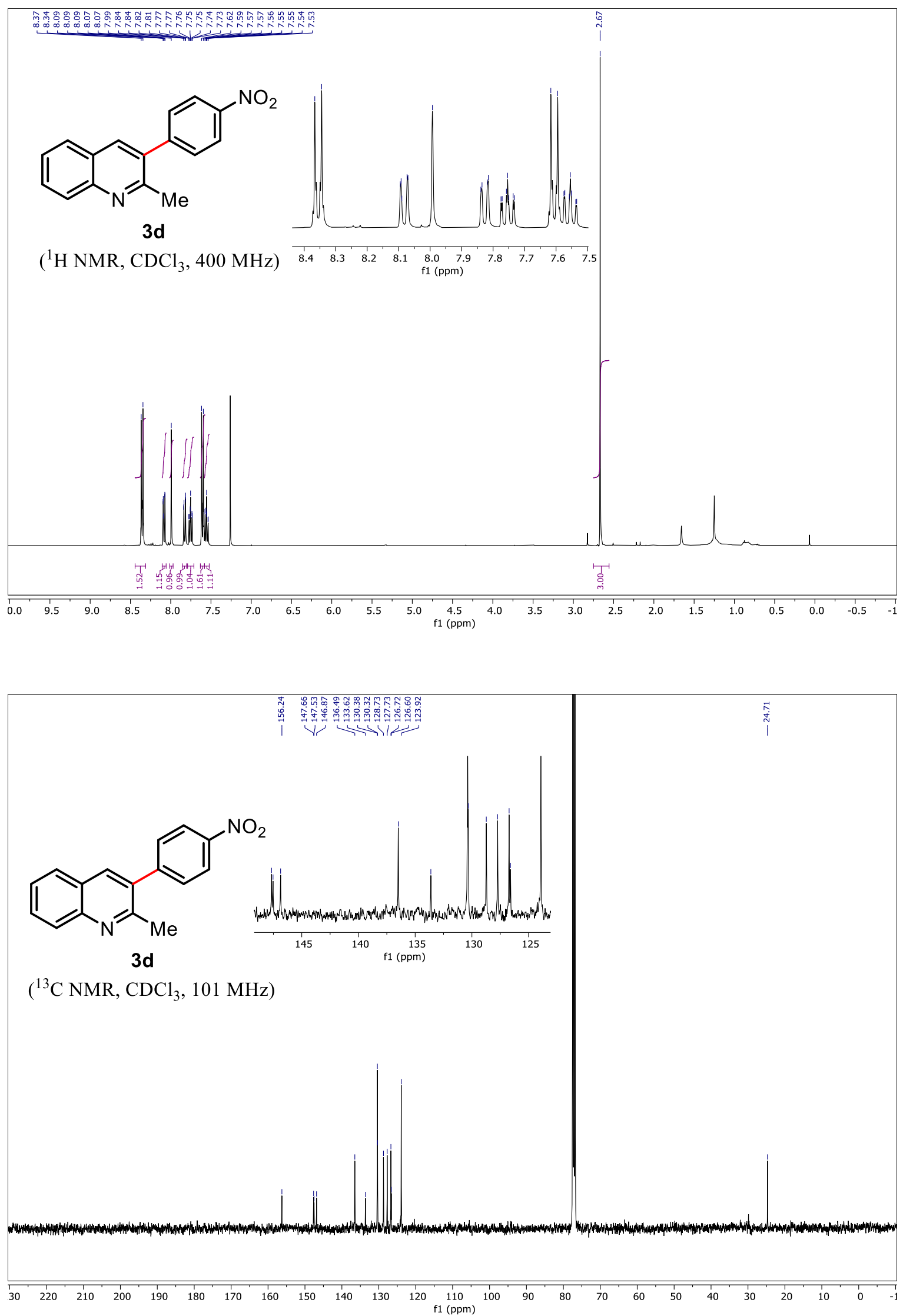

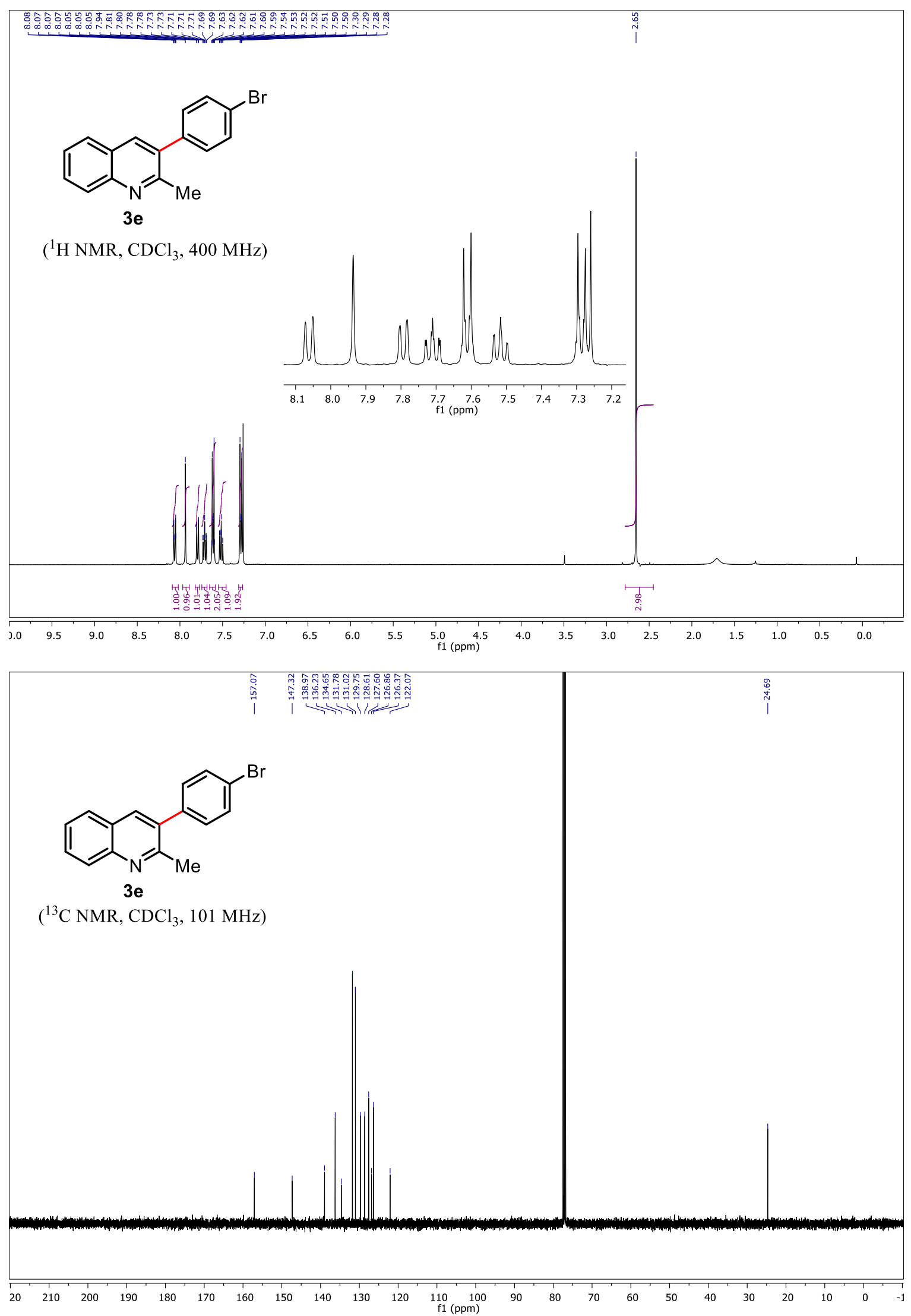

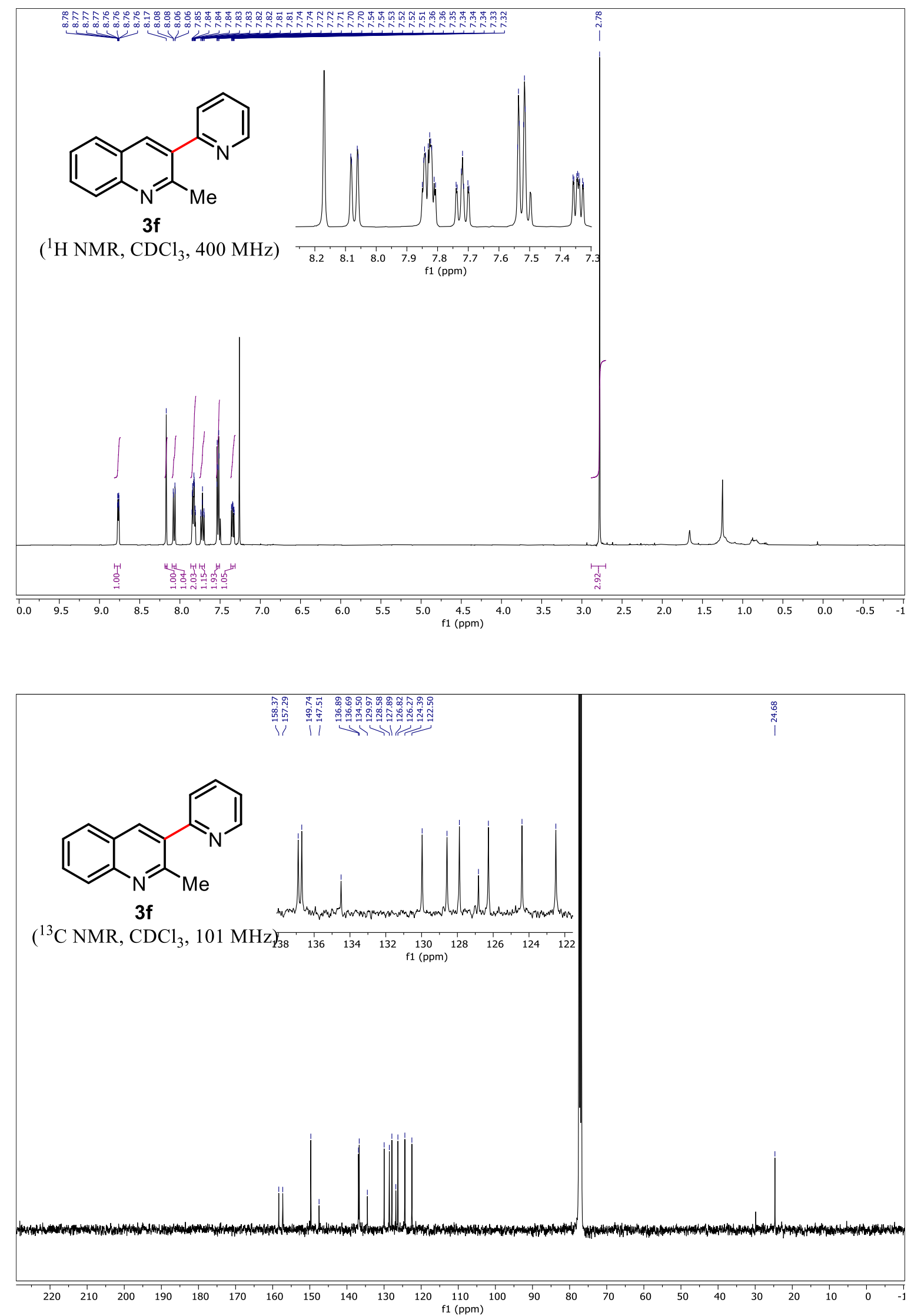

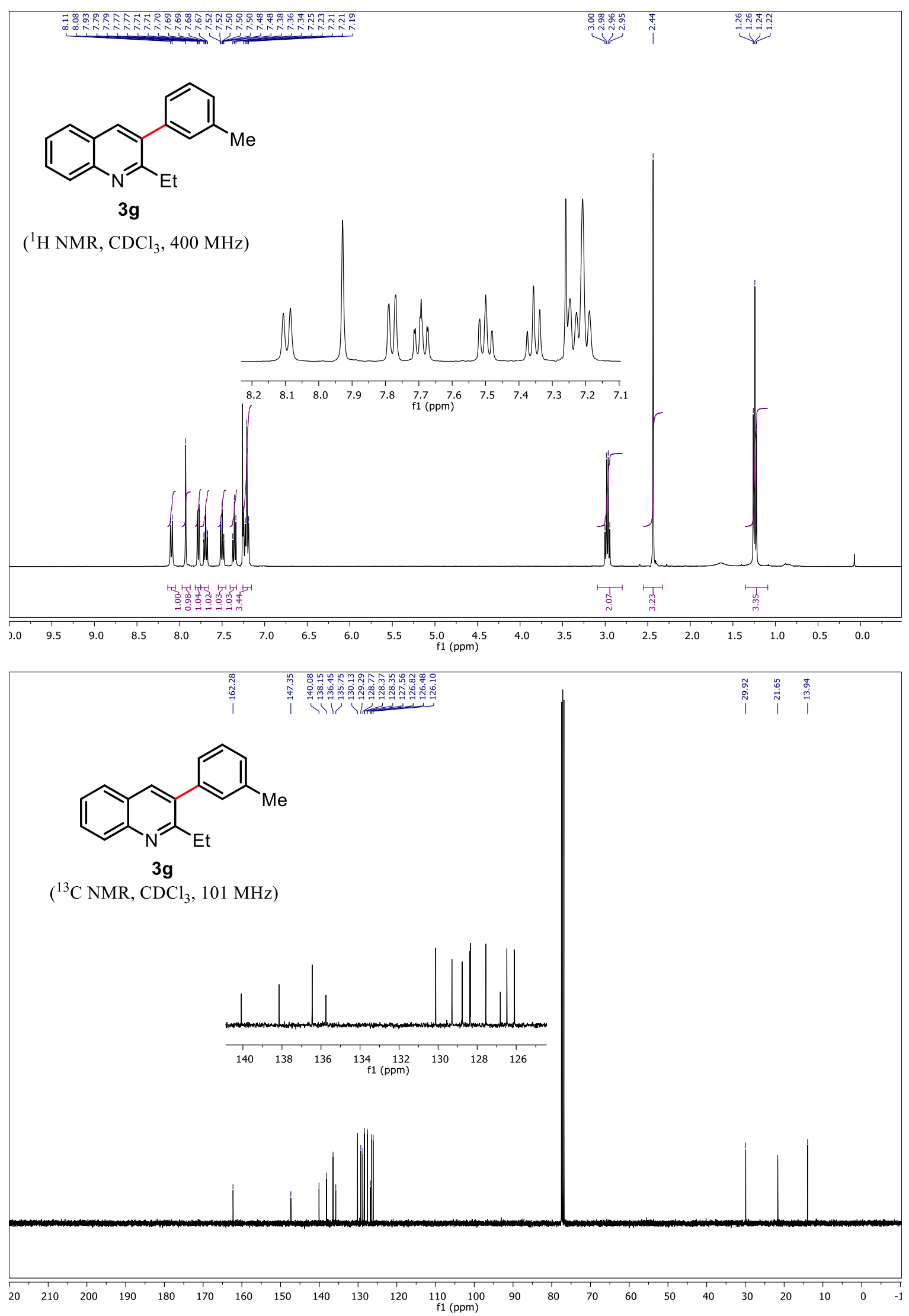

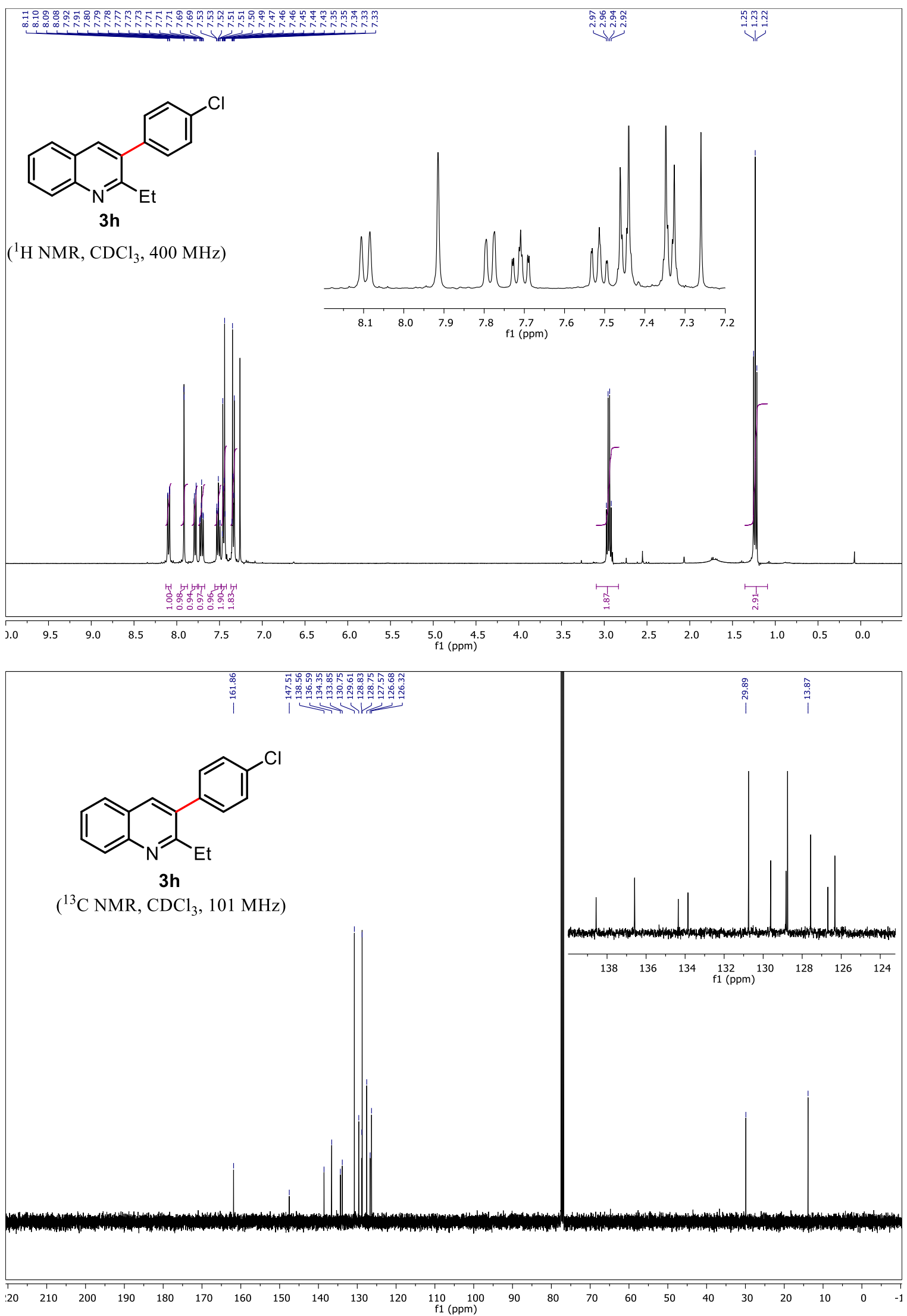

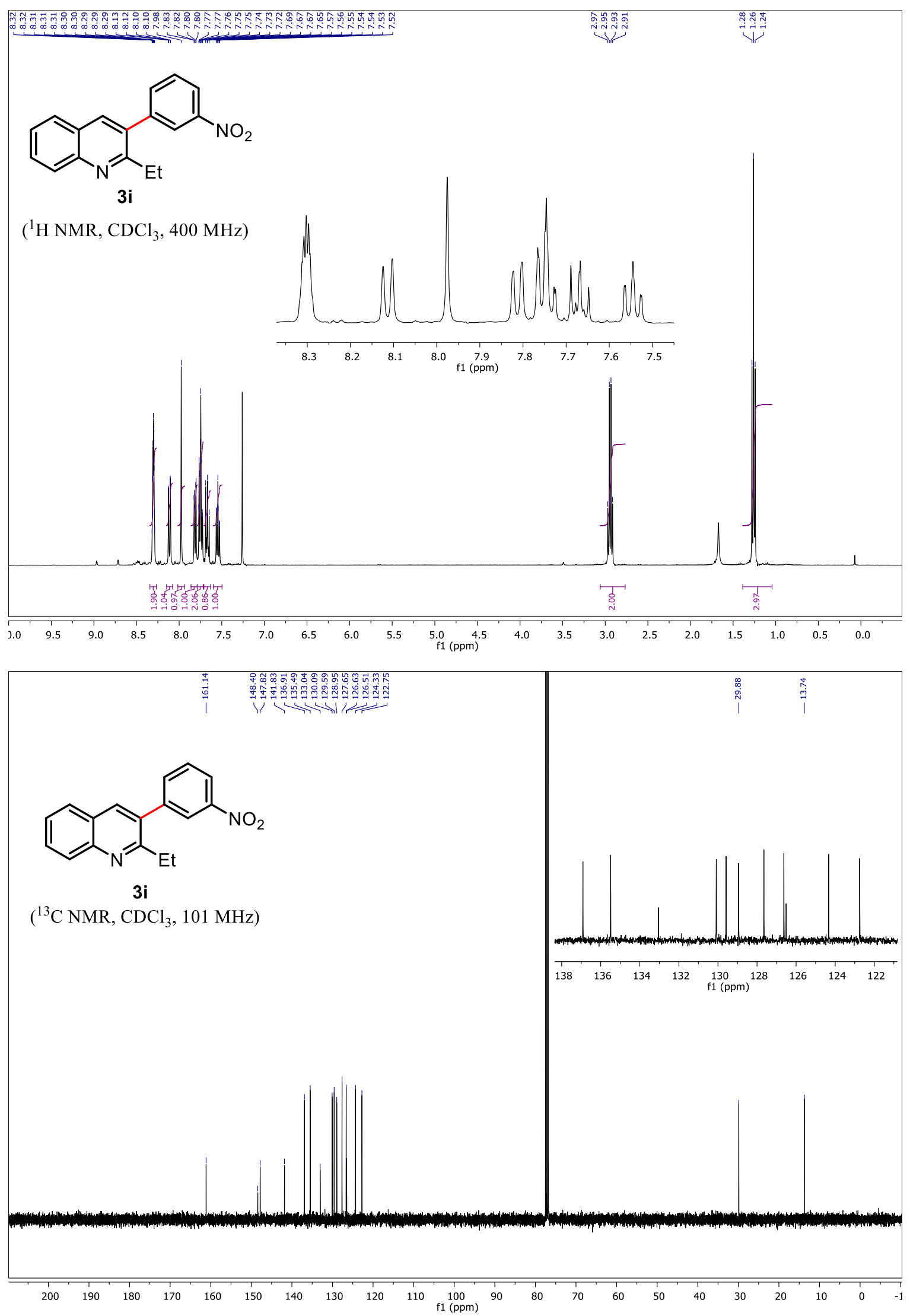

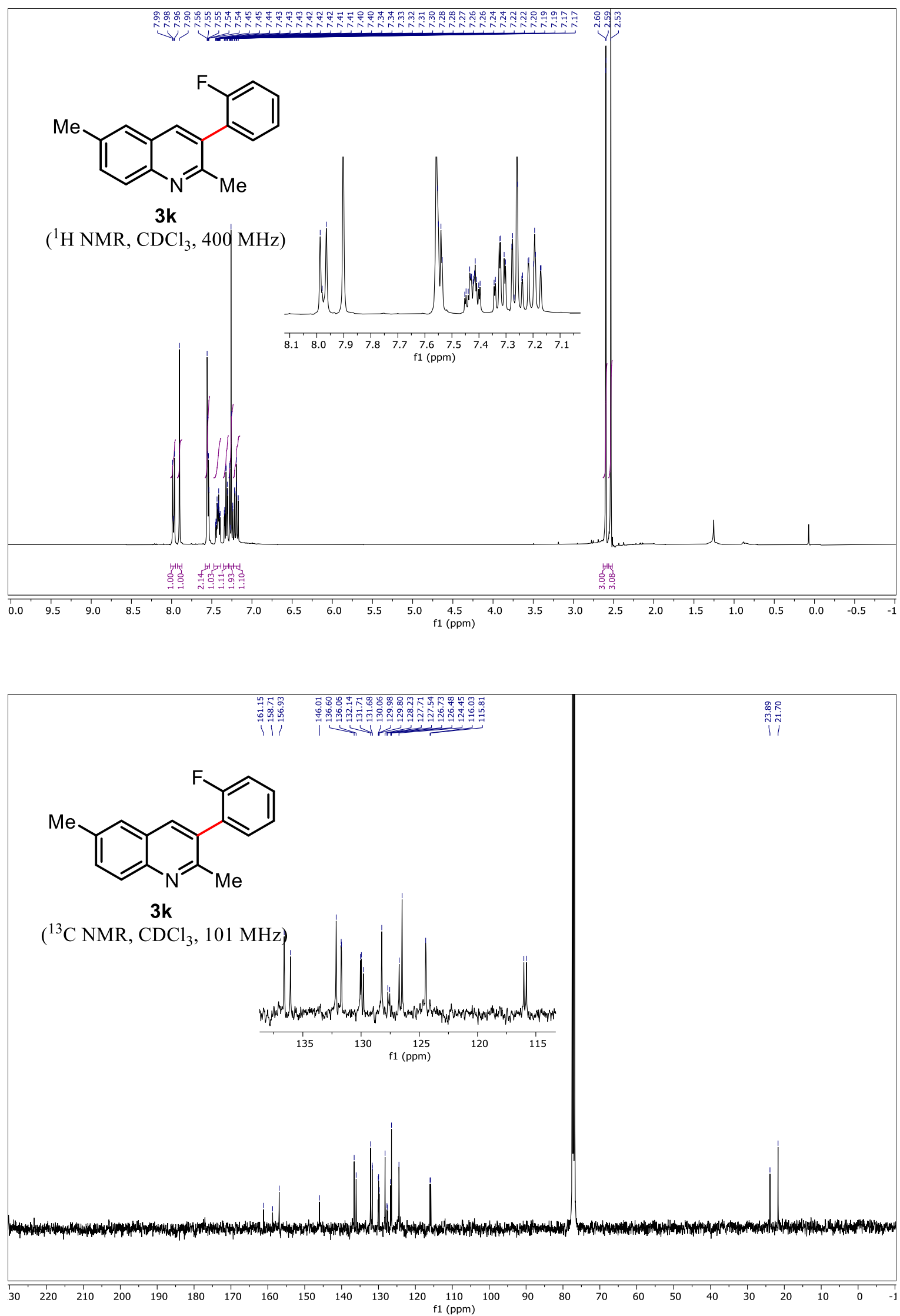

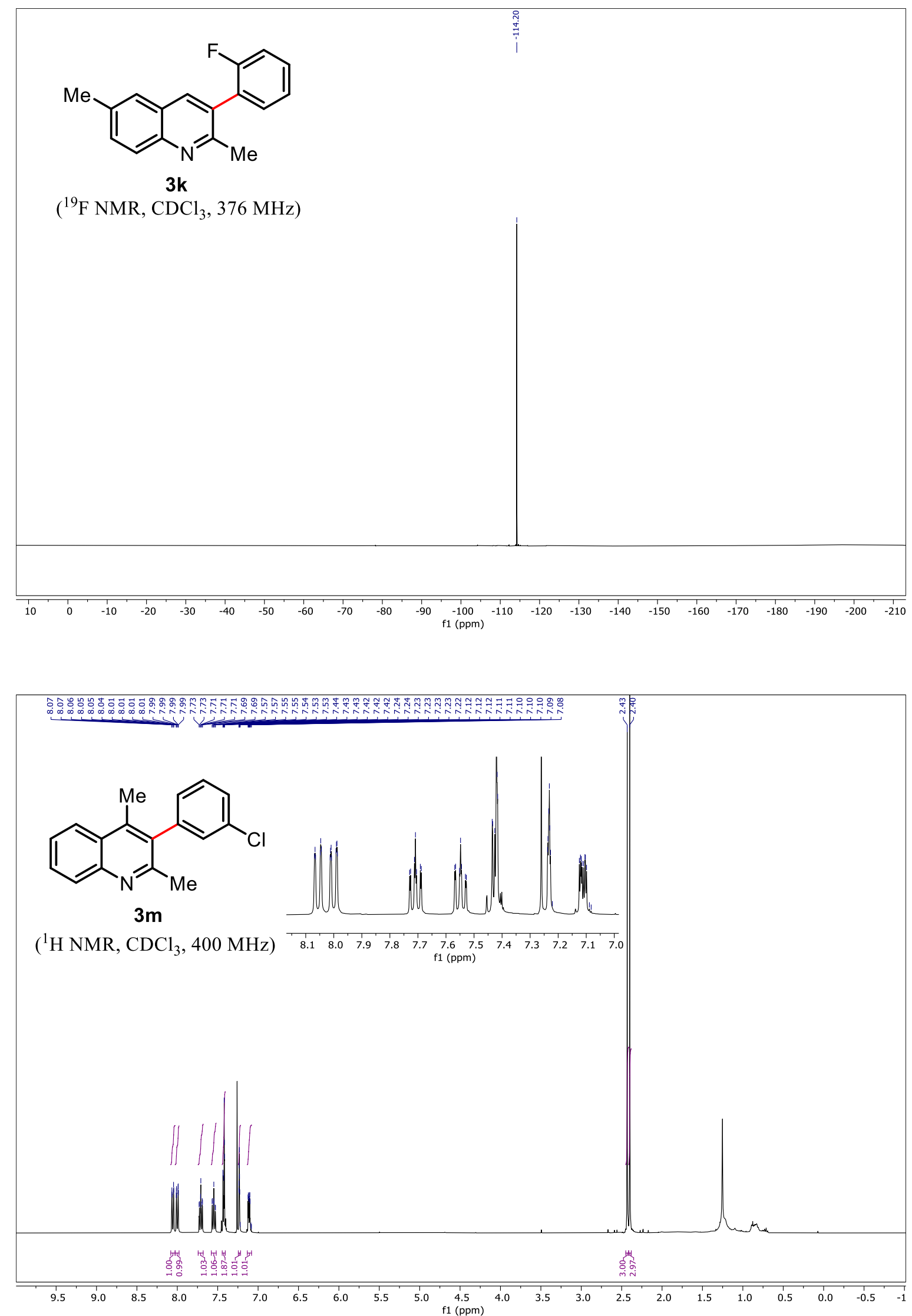

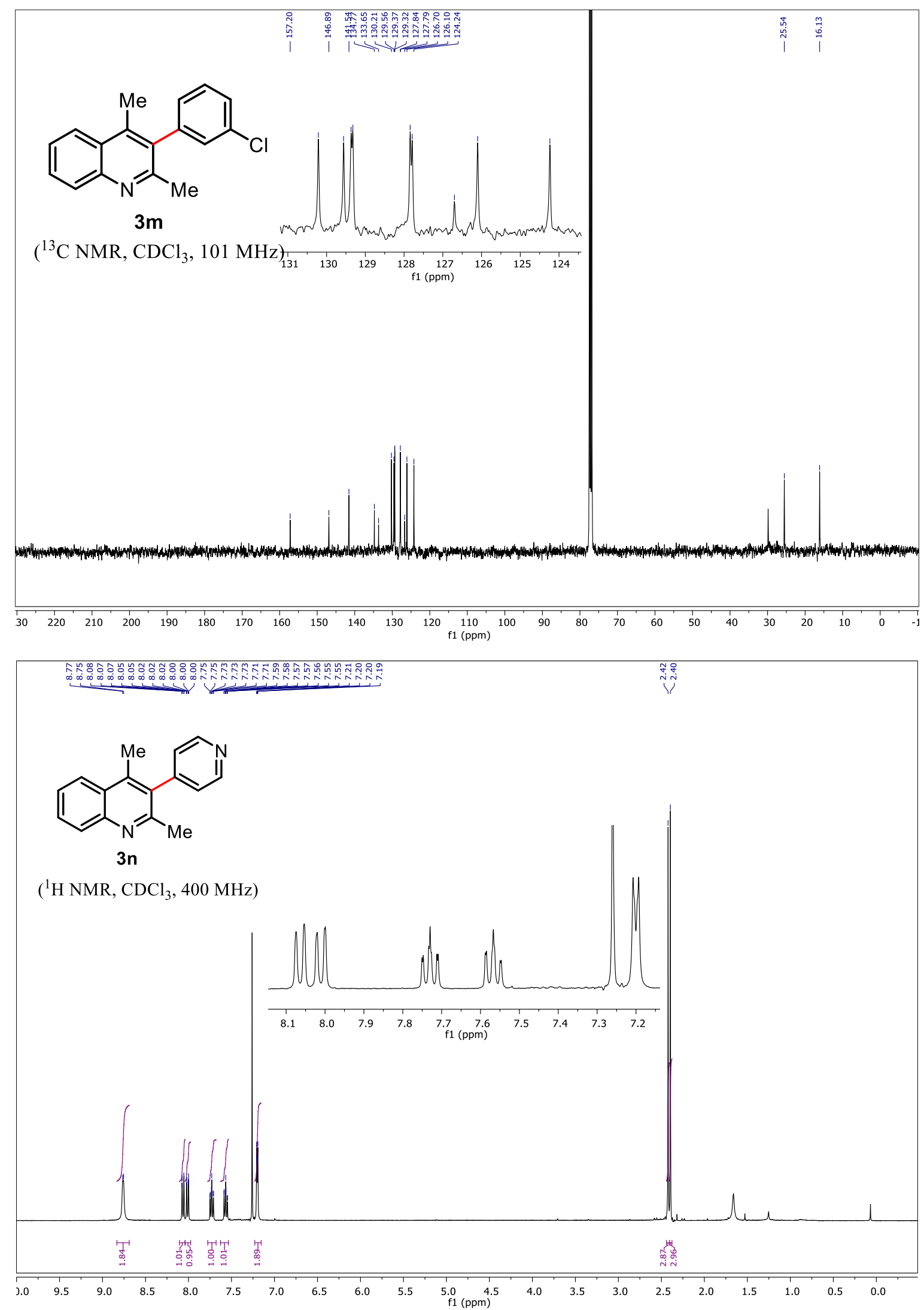

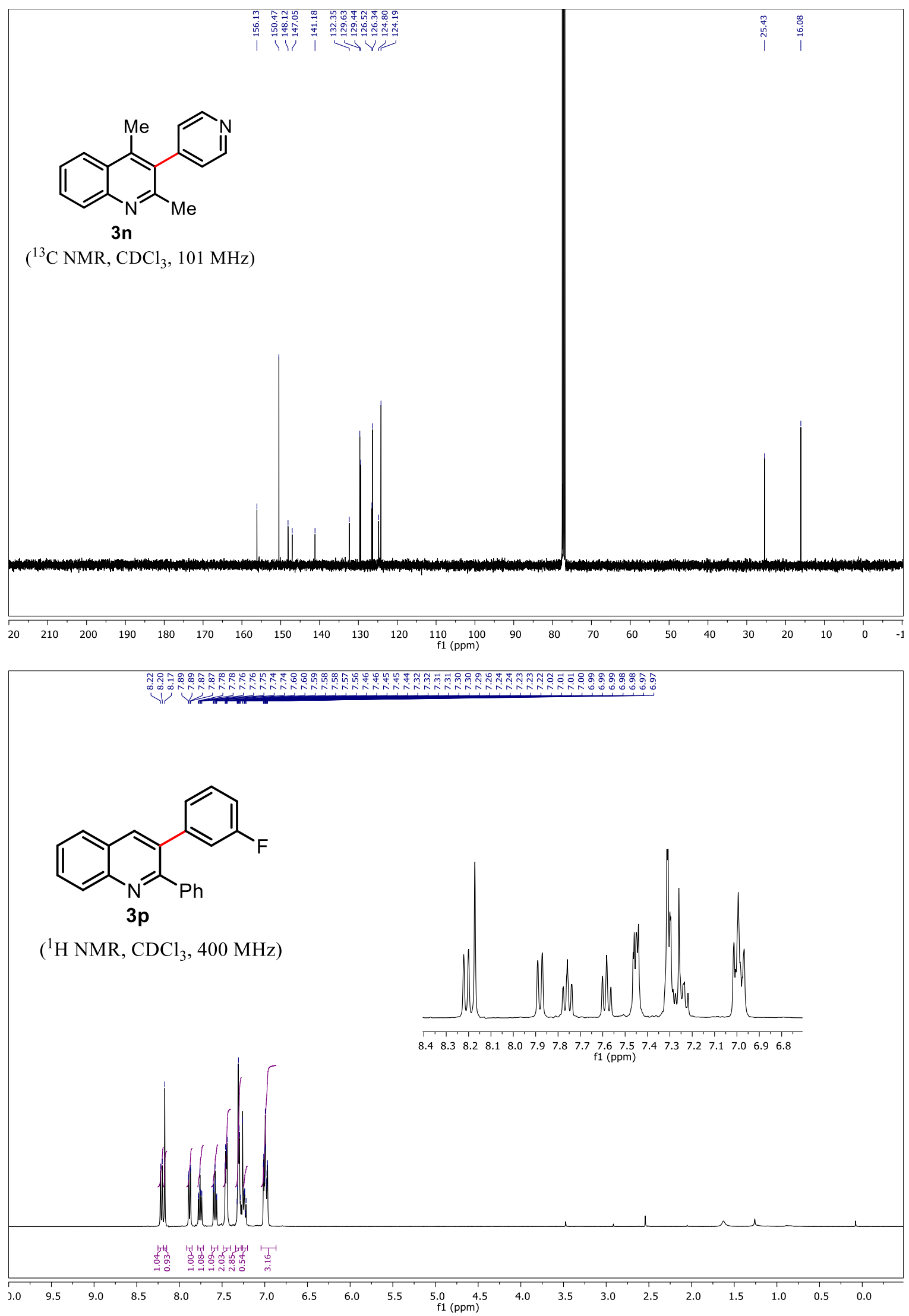

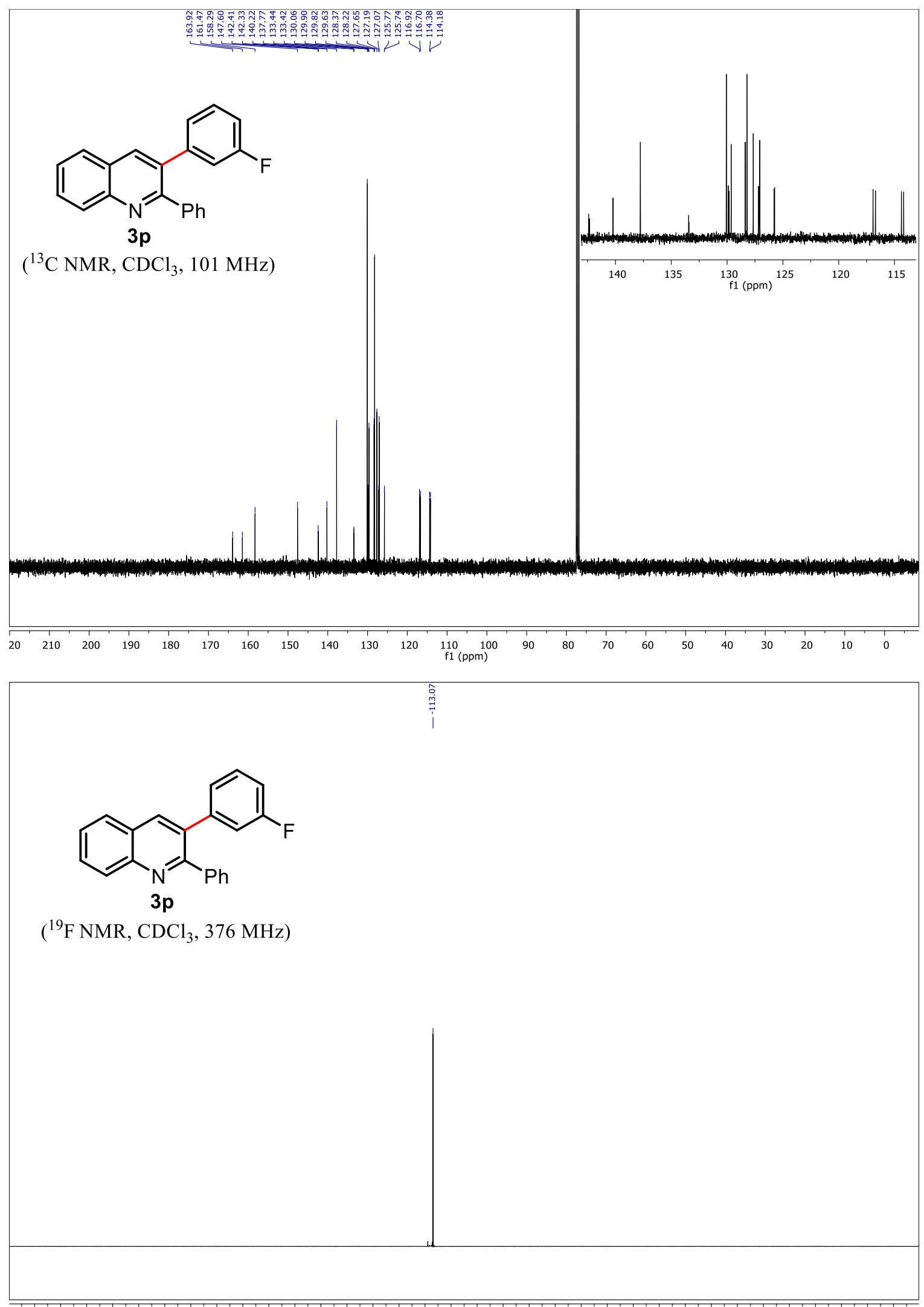

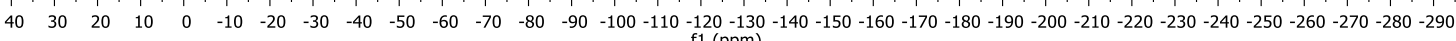



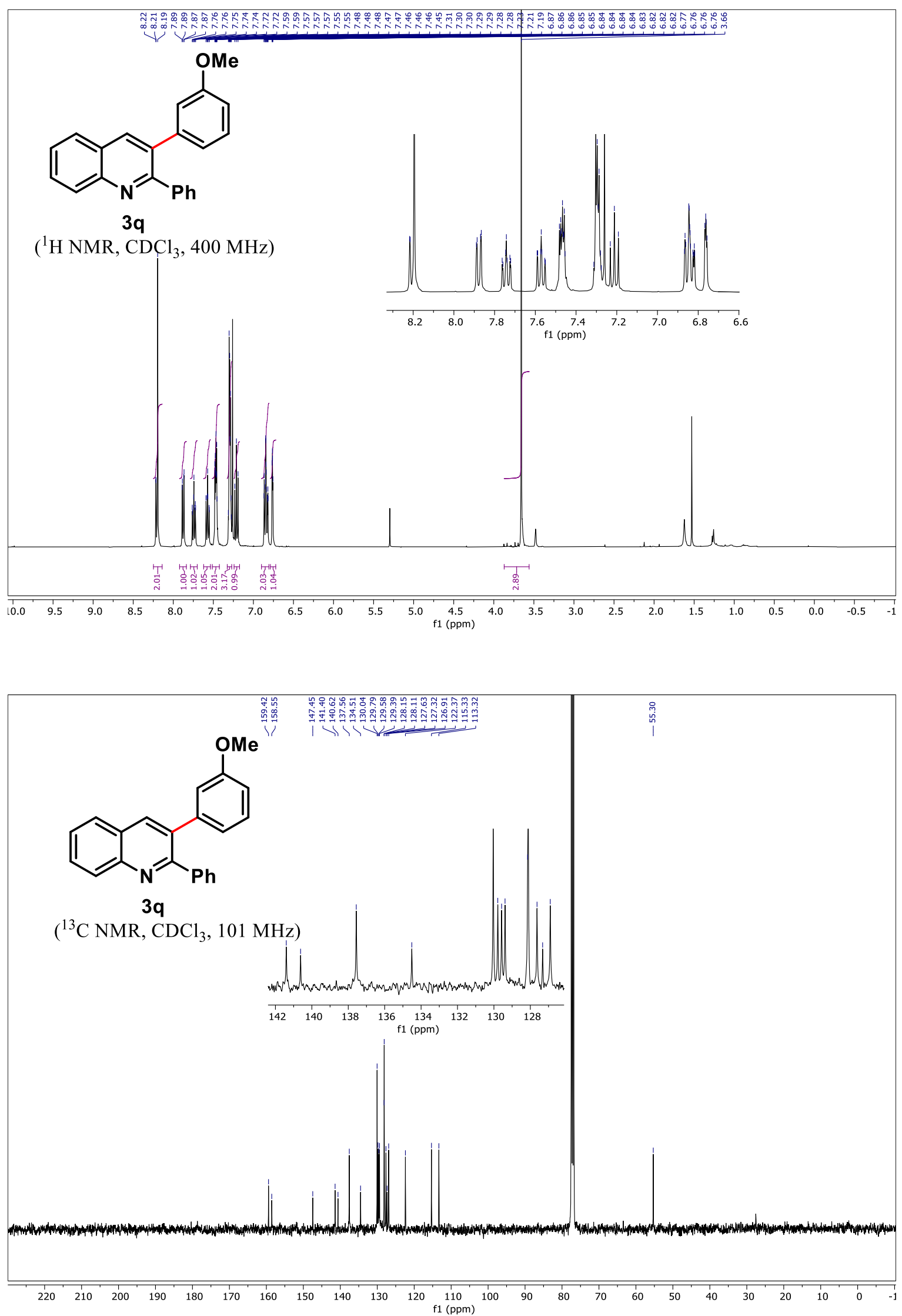

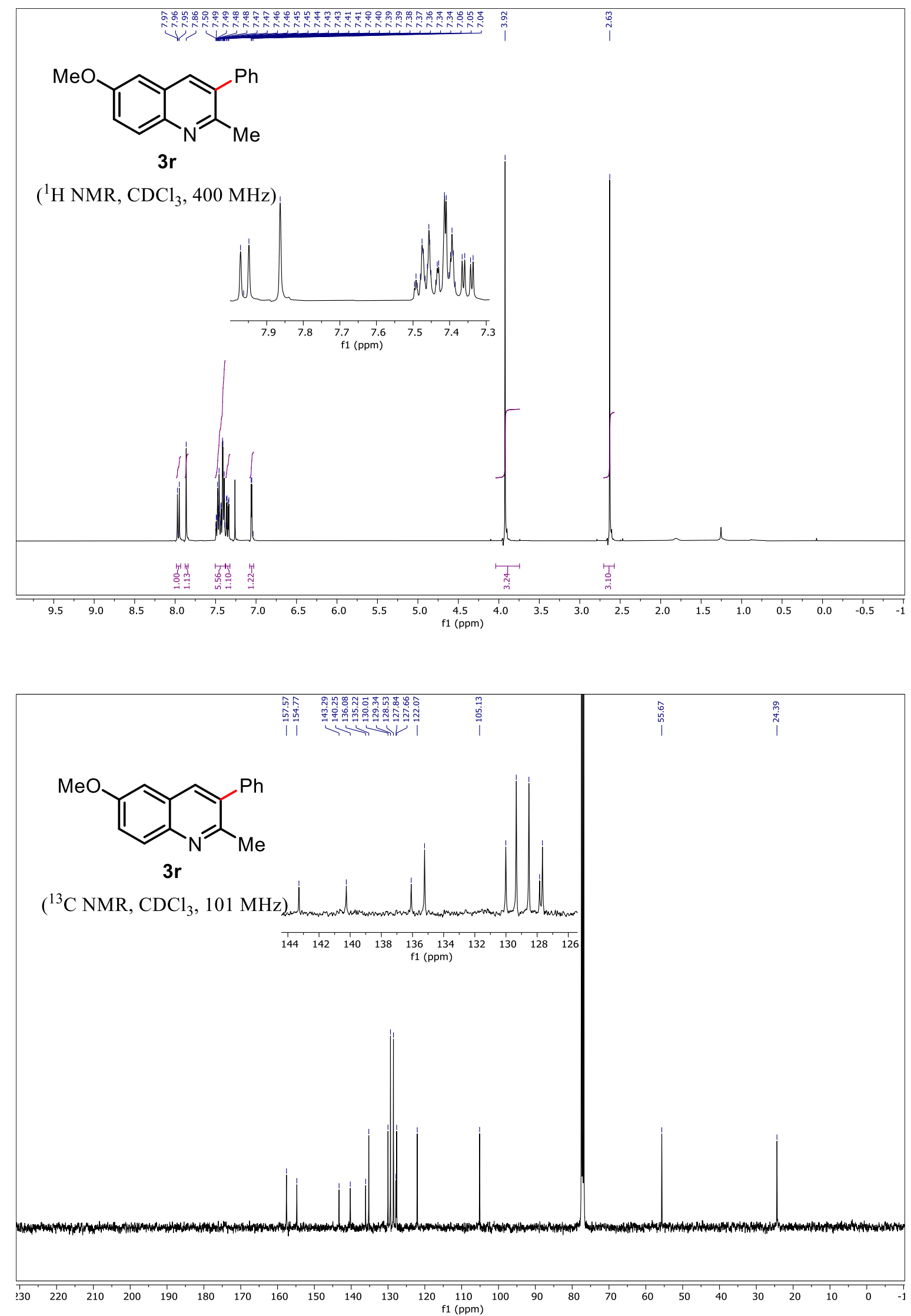

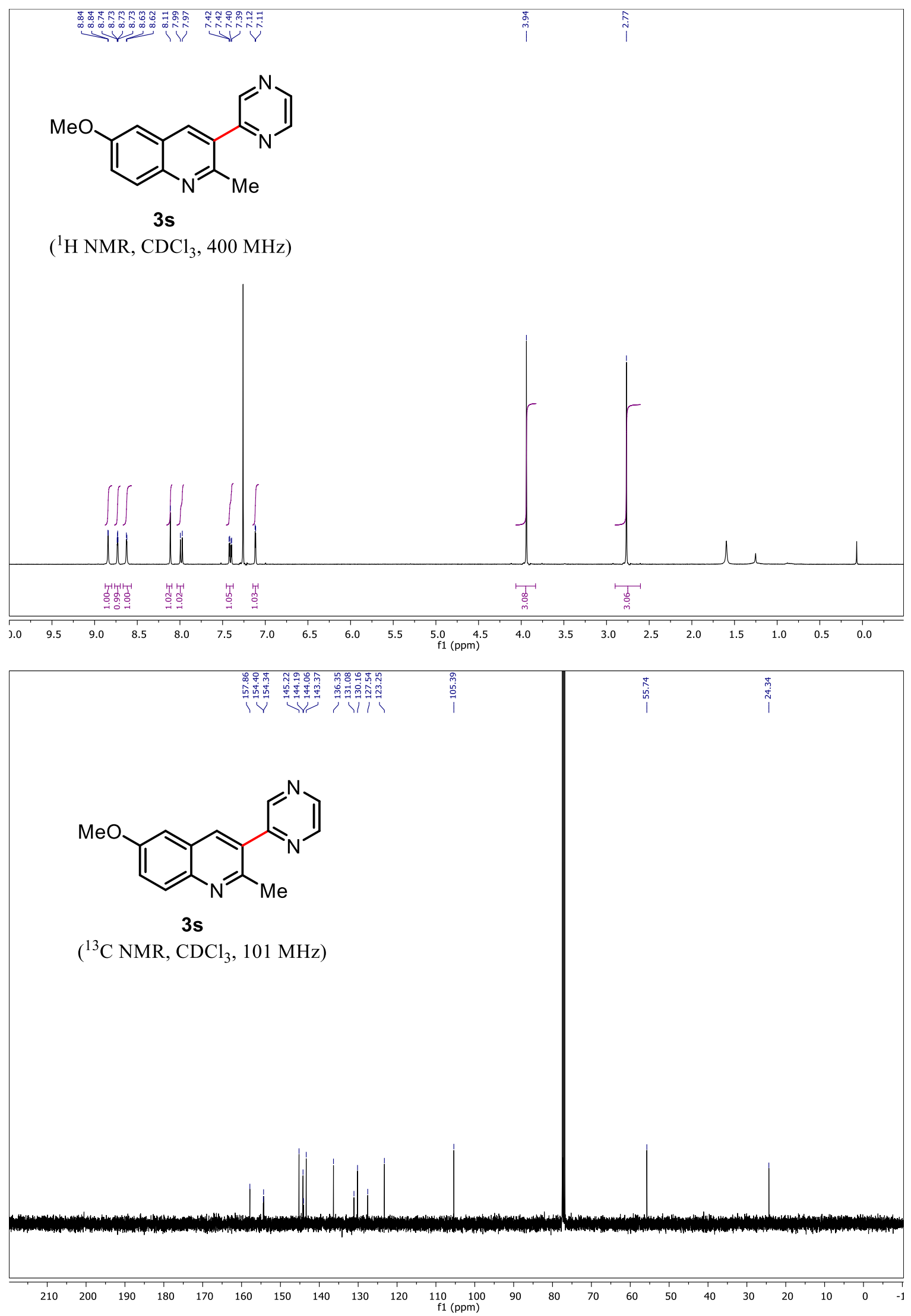

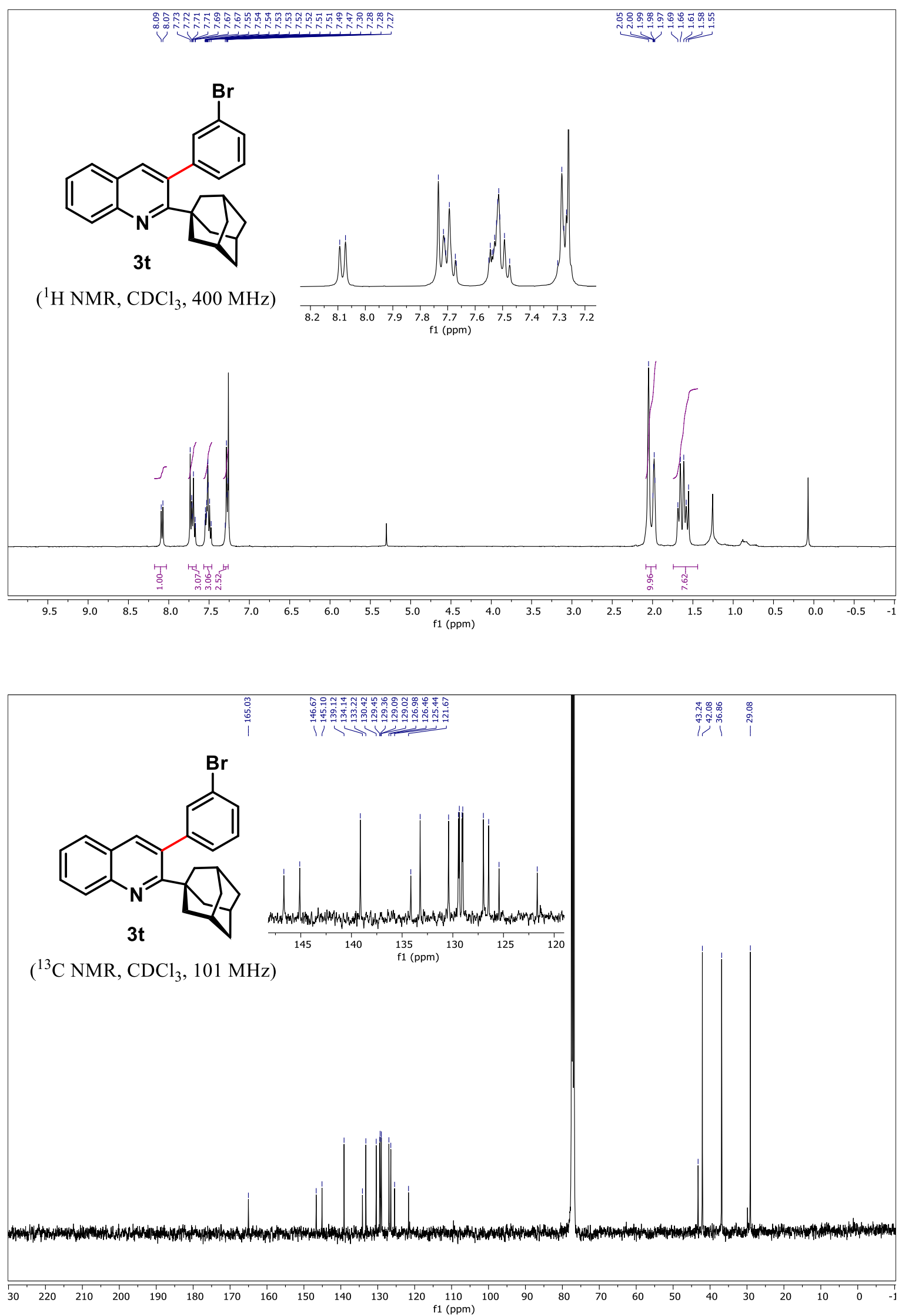
1D NOE NMR spectrum of $\mathbf{3 t}, 500 \mathrm{MHz}, \mathrm{C}_{6} \mathrm{D}_{6}$
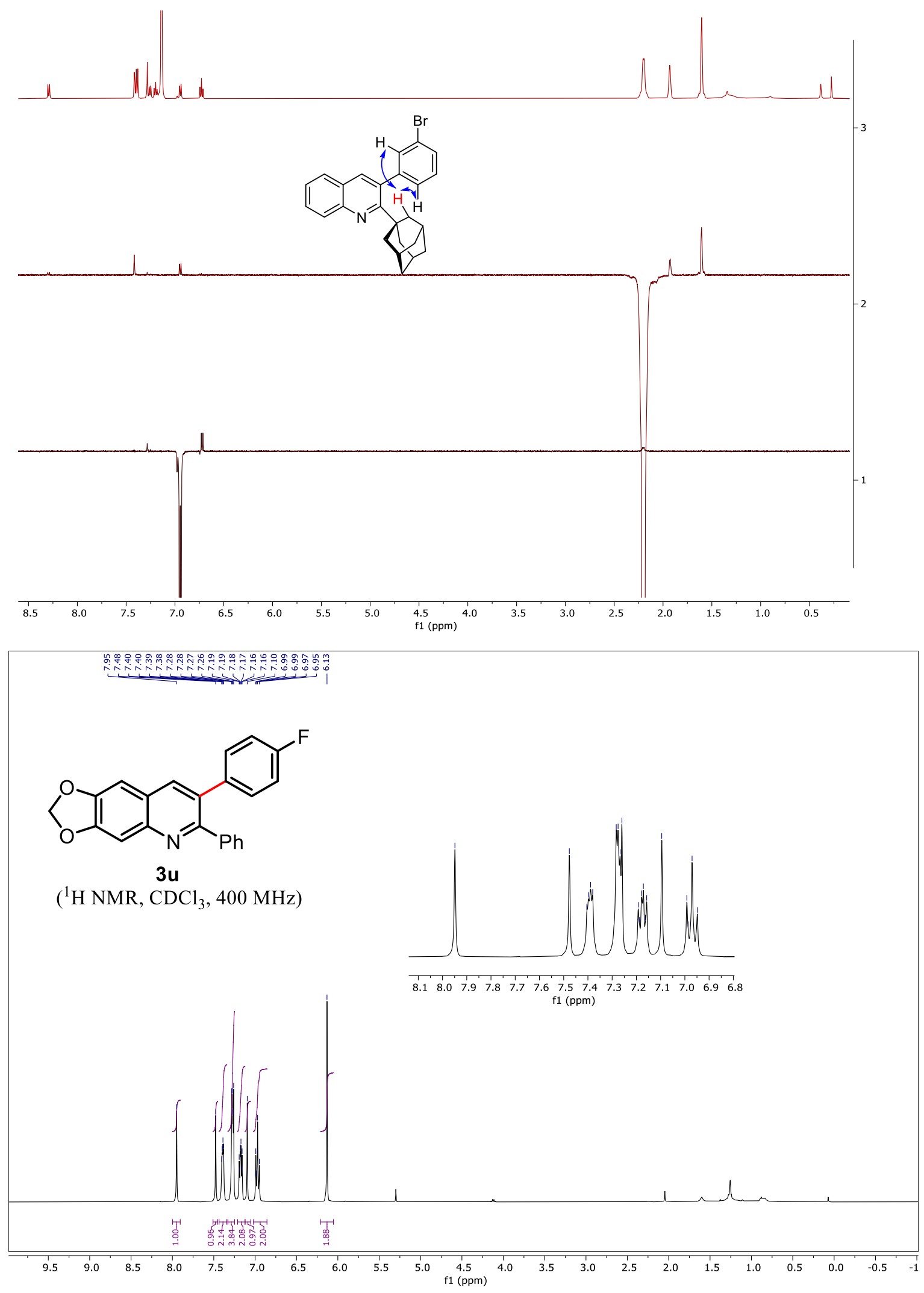

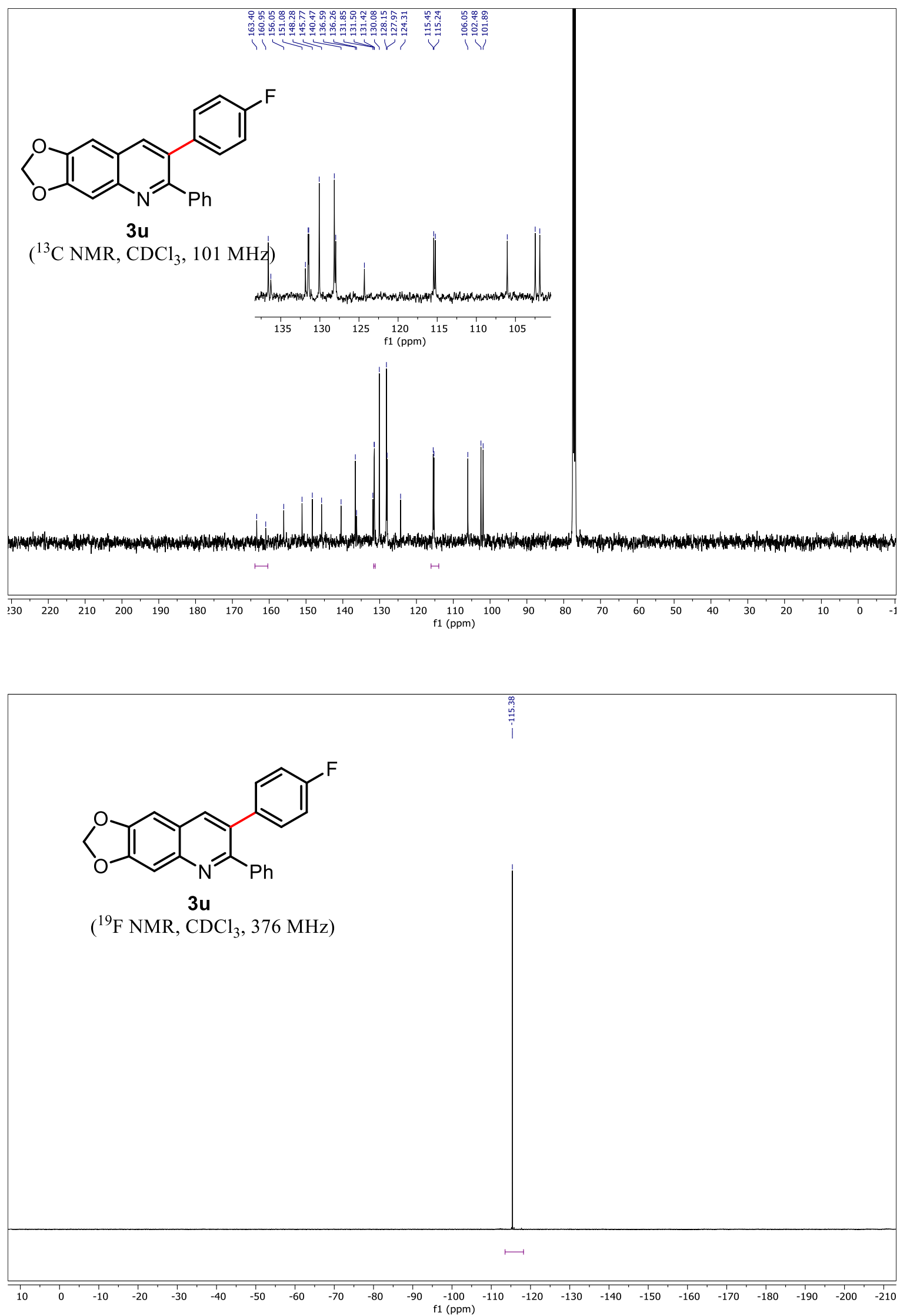
1D NOE NMR spectrum of $\mathbf{3 u}, 500 \mathrm{MHz}, \mathrm{CDCl}_{3}$
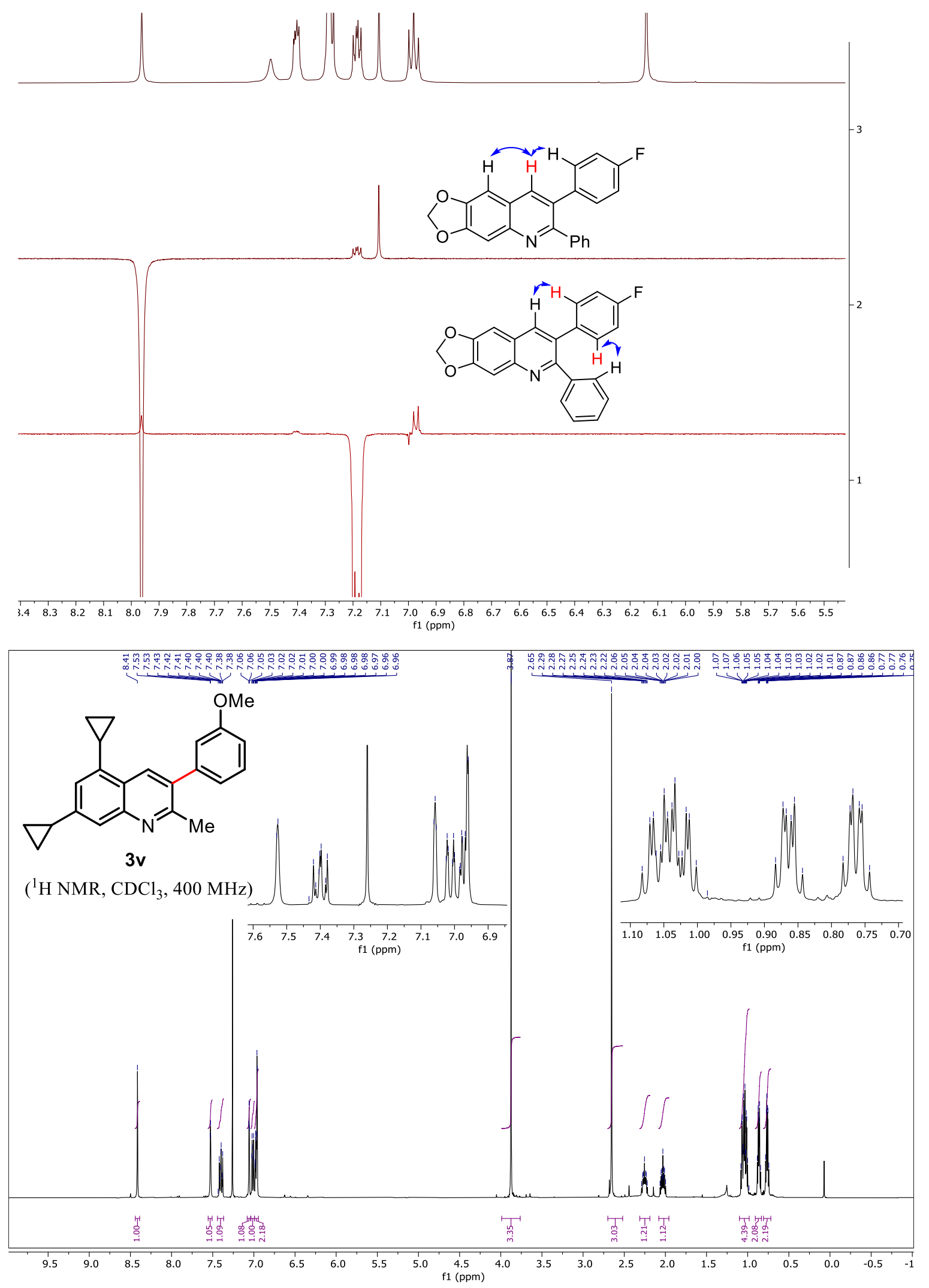


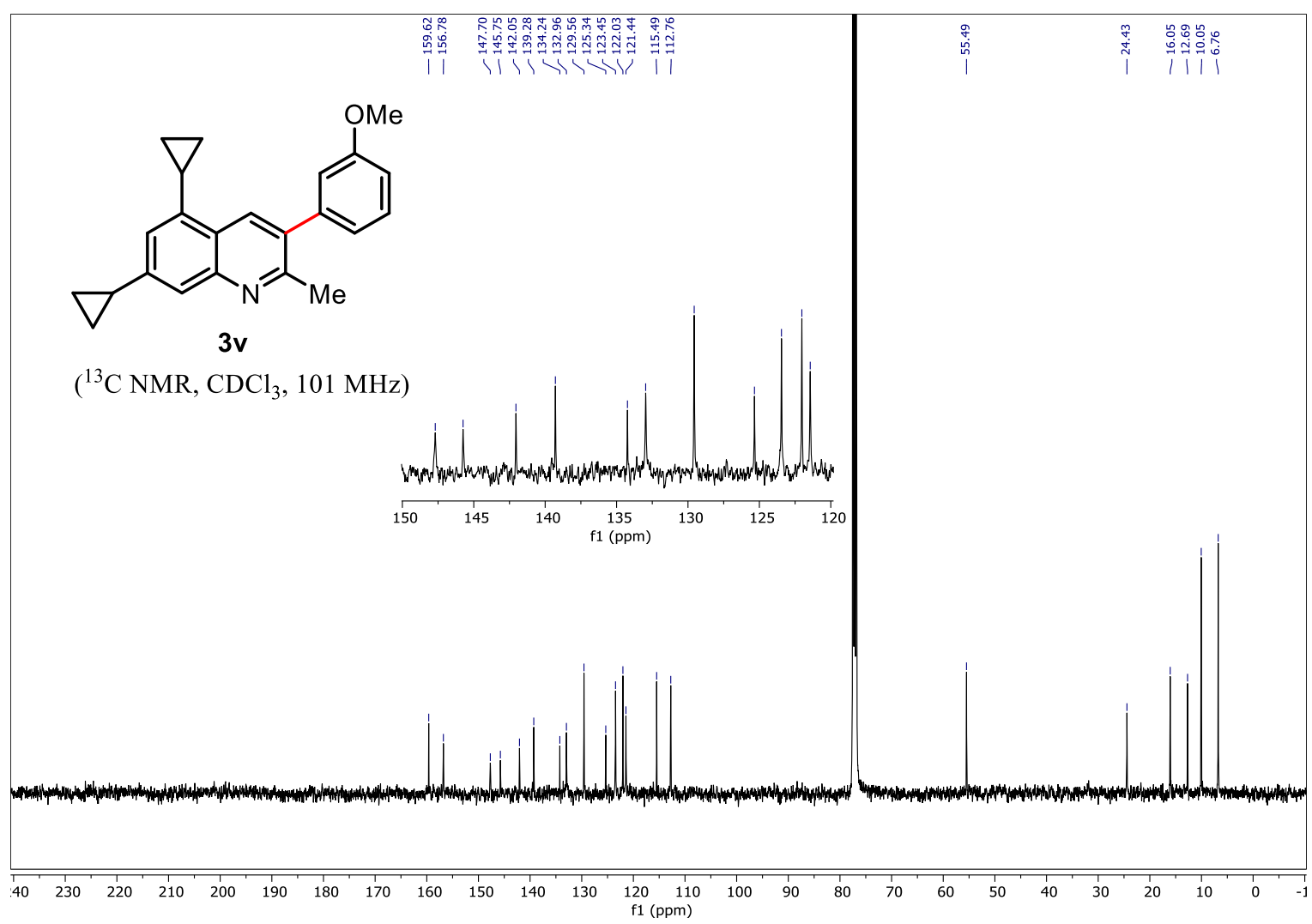

1D NOE NMR spectrum of $\mathbf{3 v}, 500 \mathrm{MHz}, \mathrm{CDCl}_{3}$
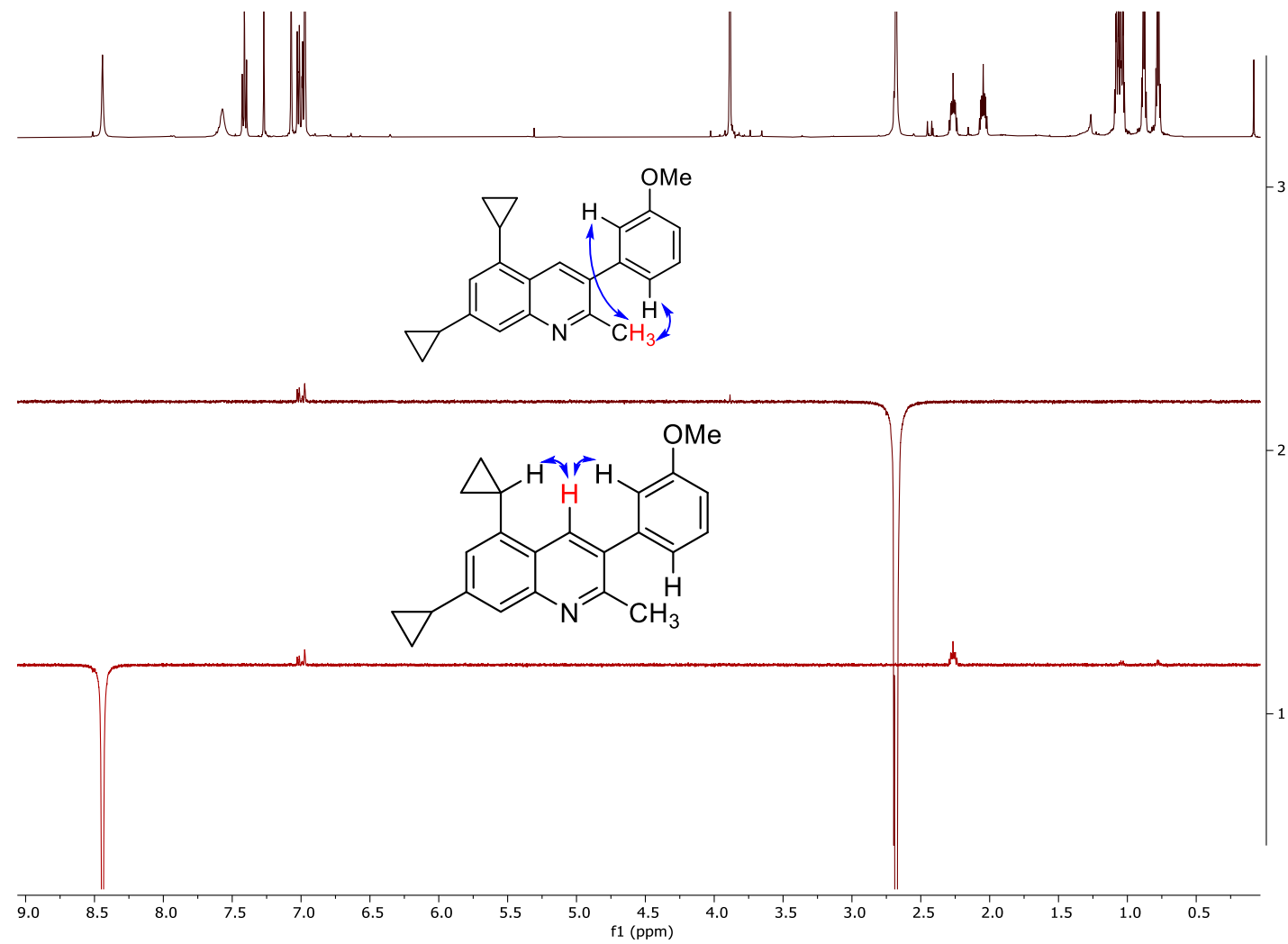

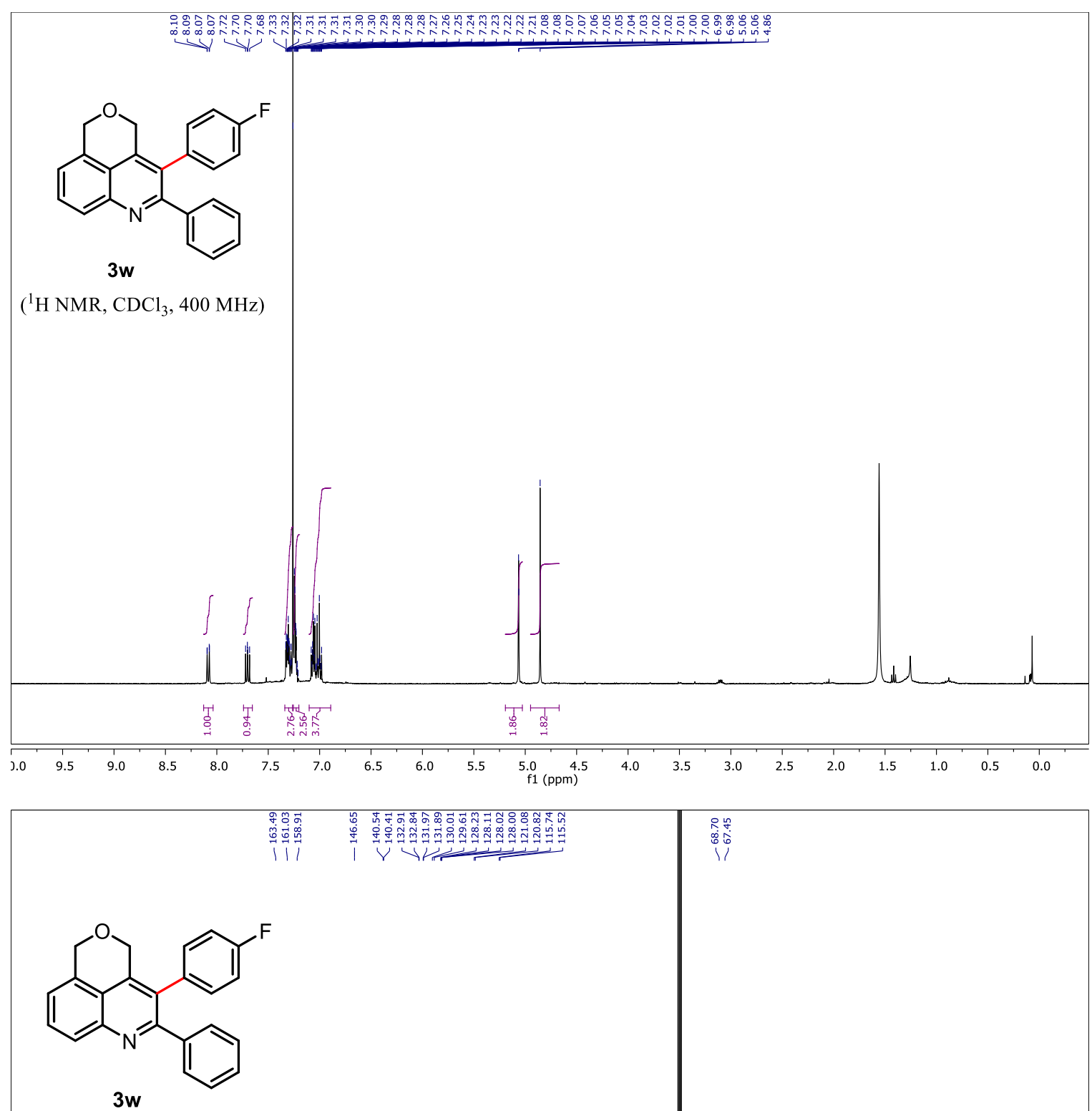

$\left({ }^{13} \mathrm{C}\right.$ NMR, $\left.\mathrm{CDCl}_{3}, 101 \mathrm{MHz}\right)$

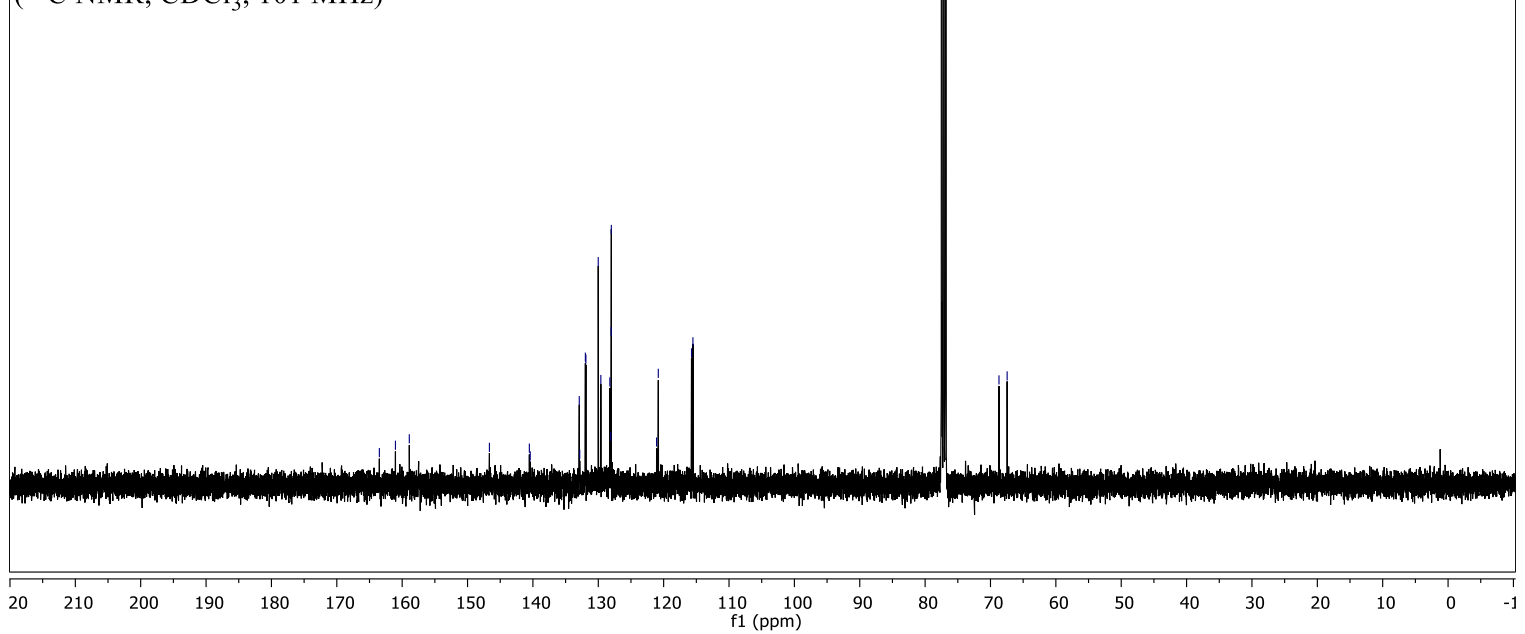




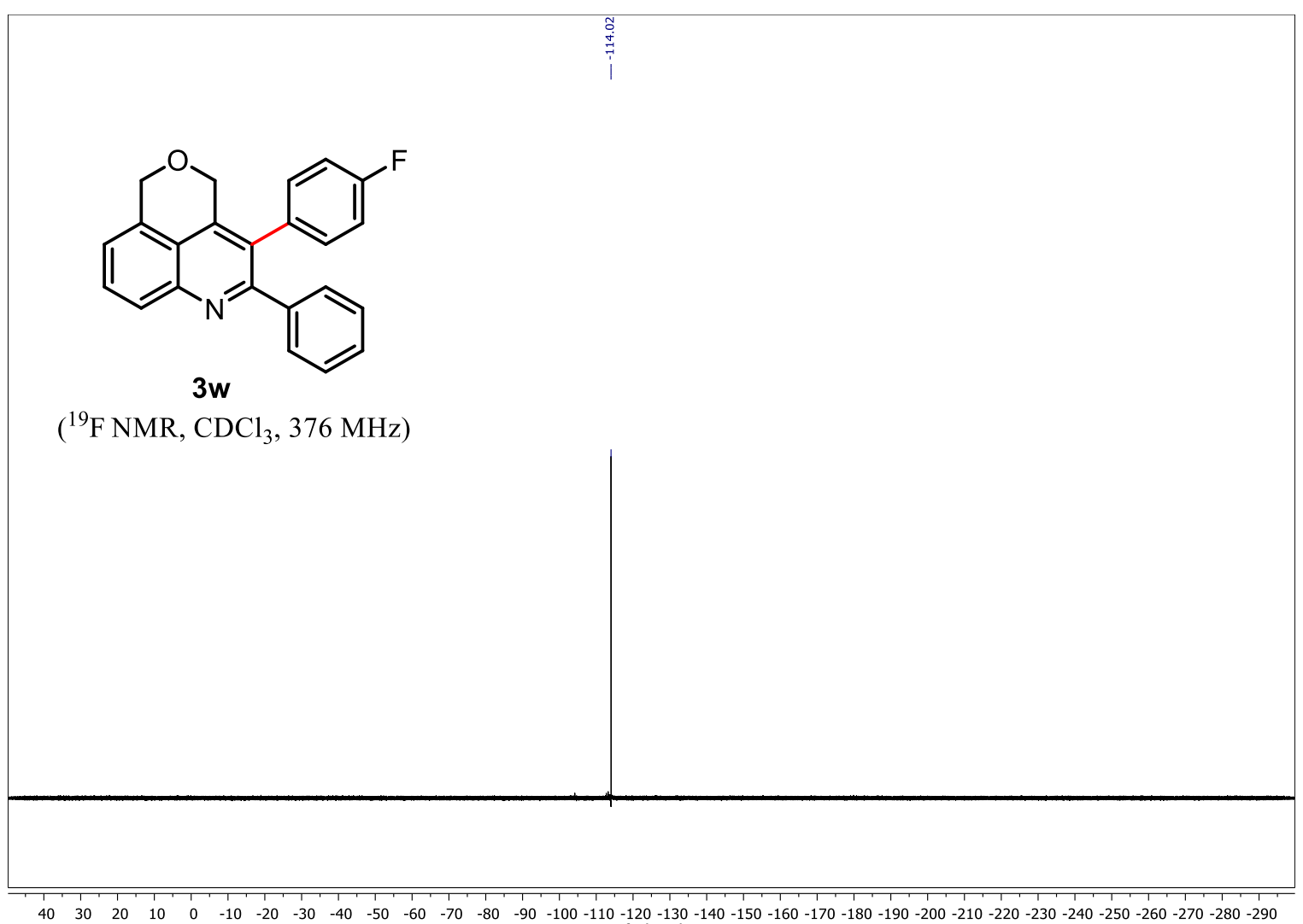

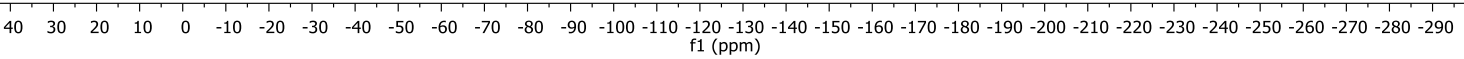

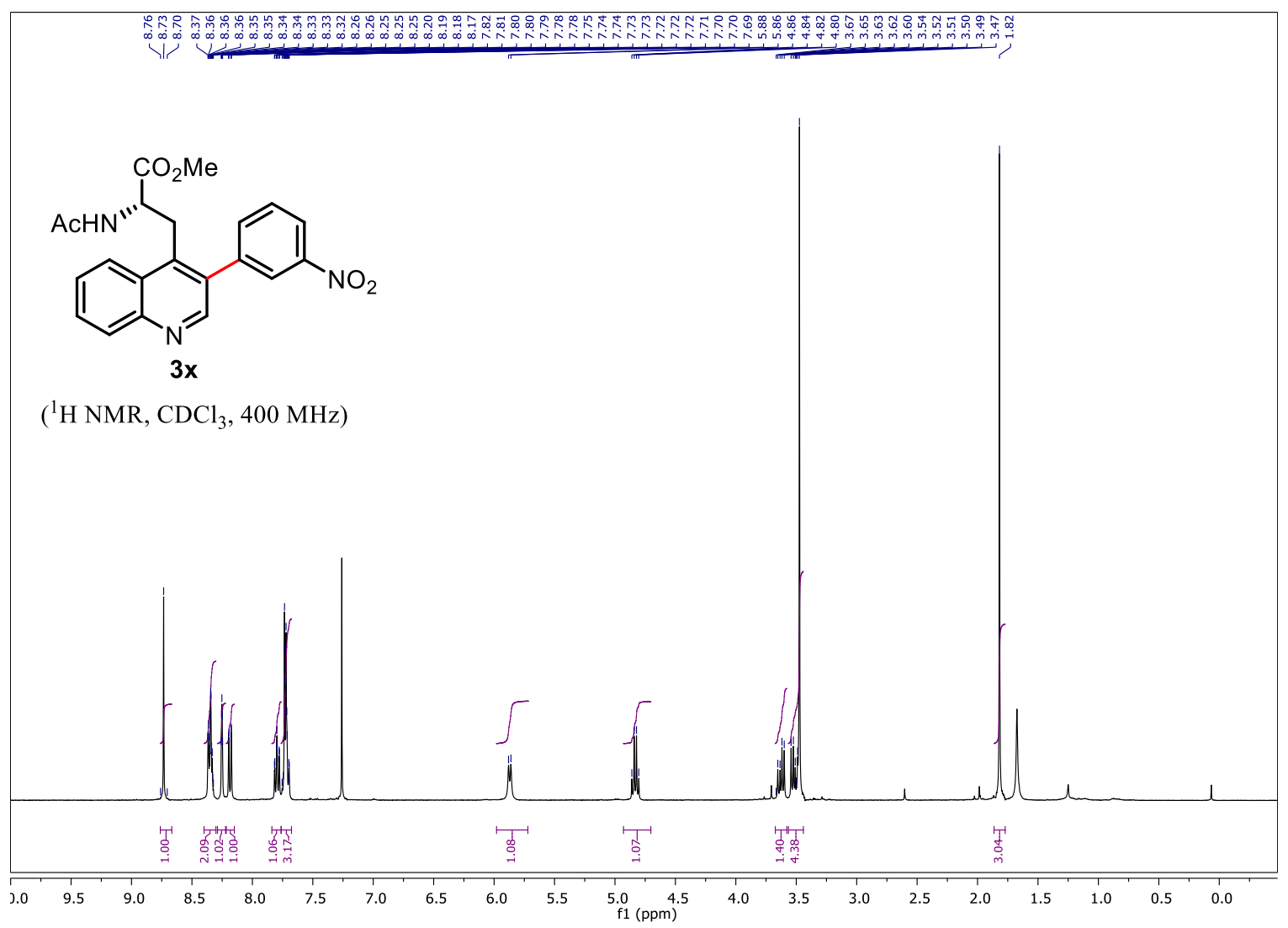



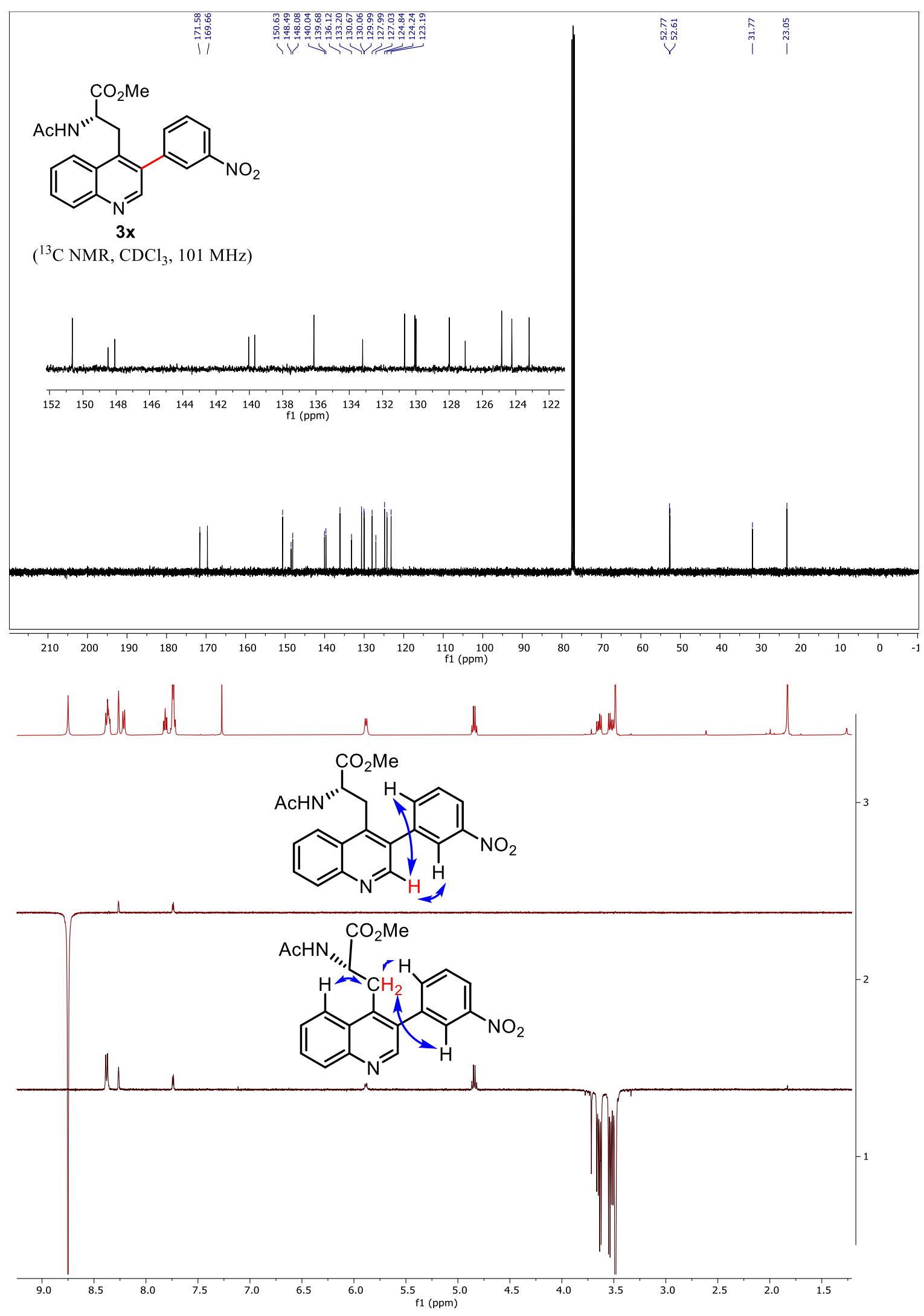

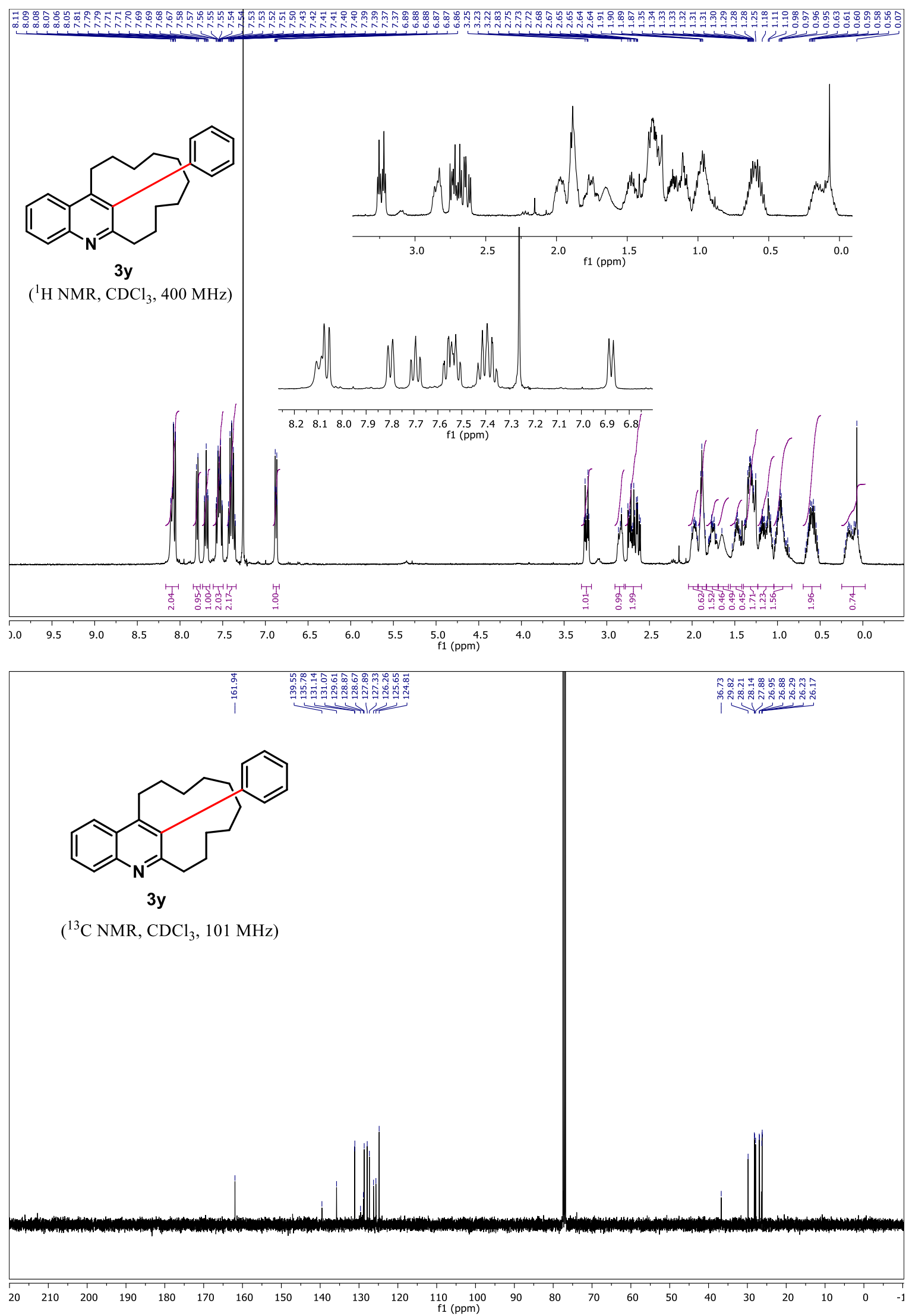

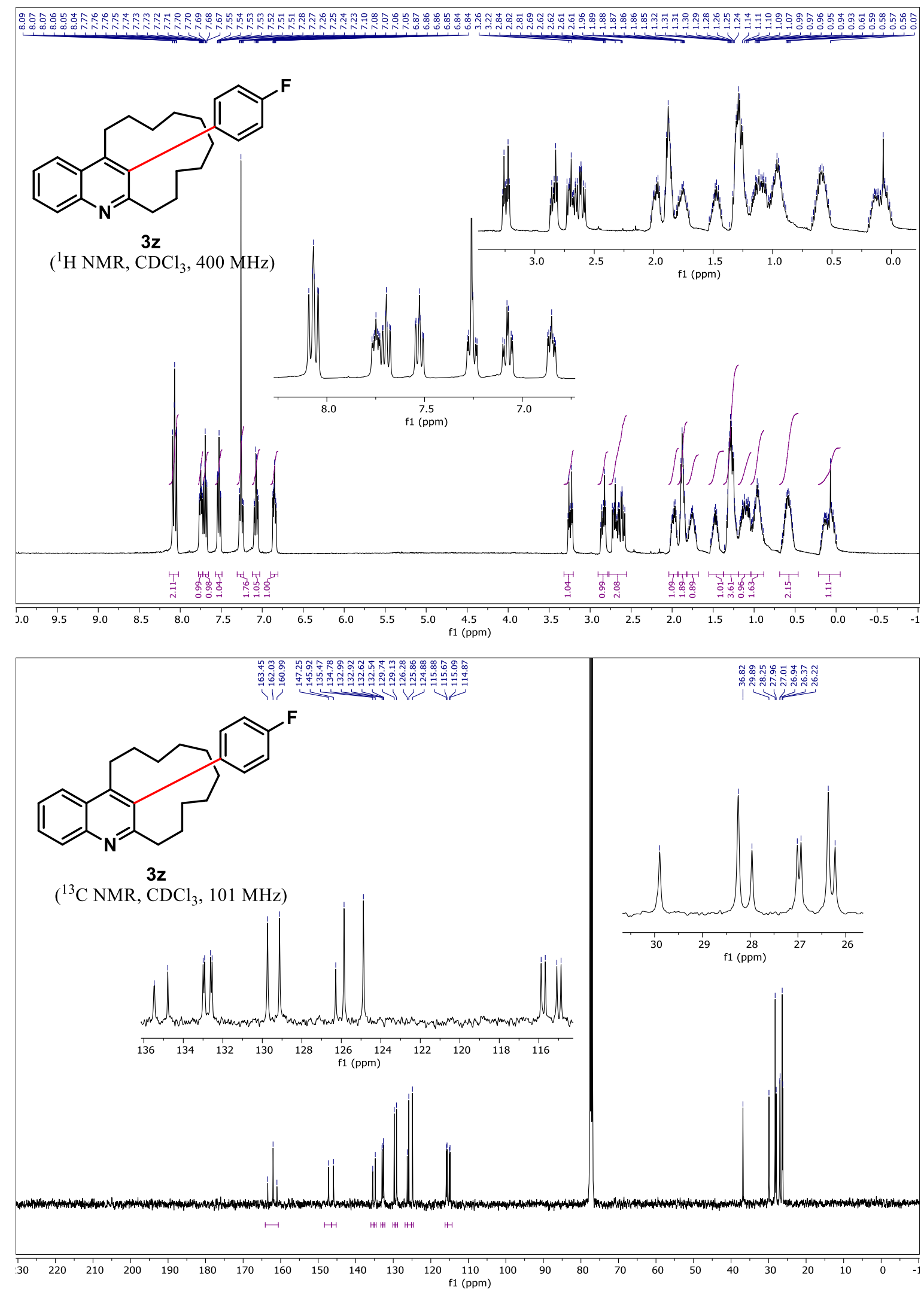

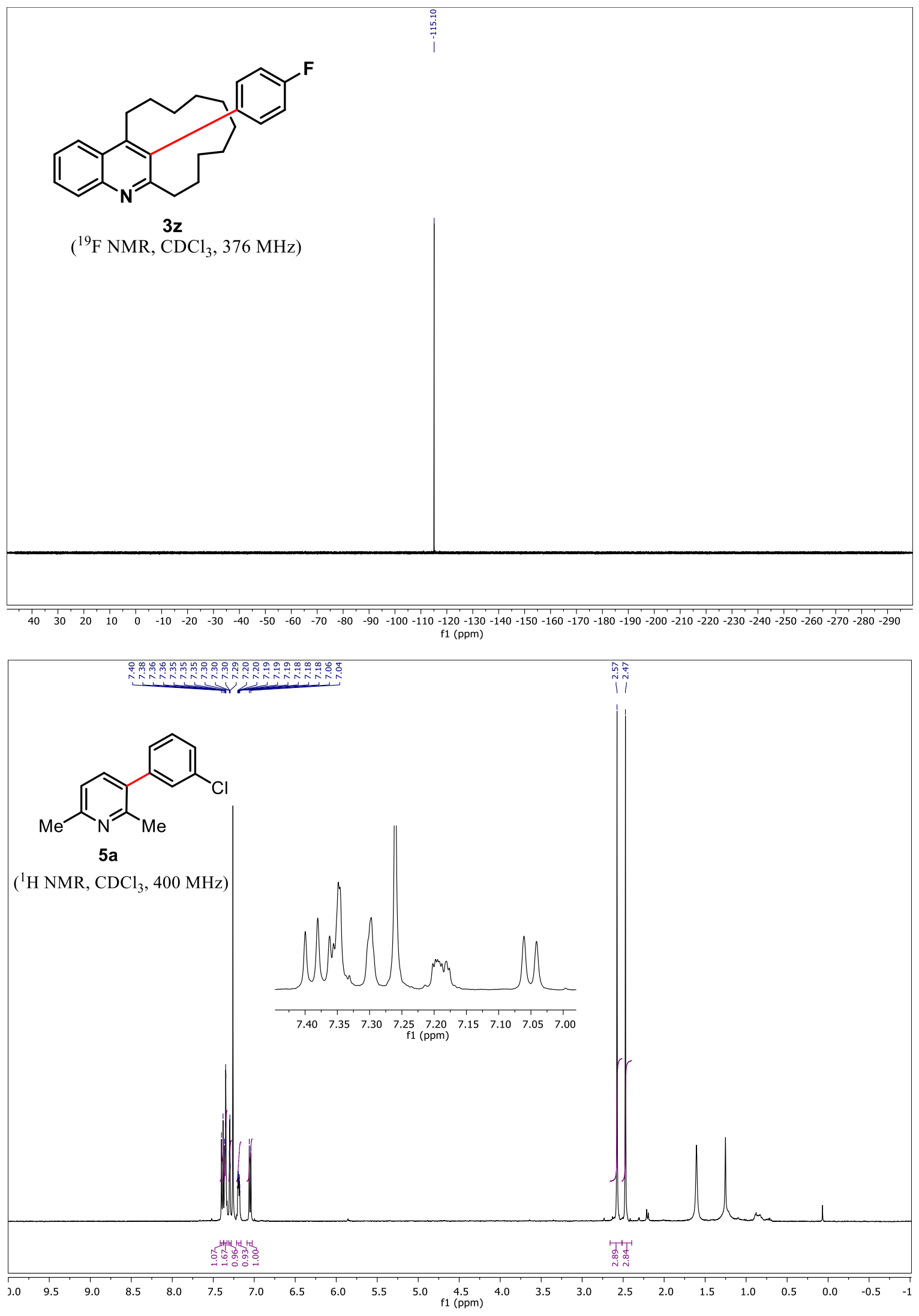

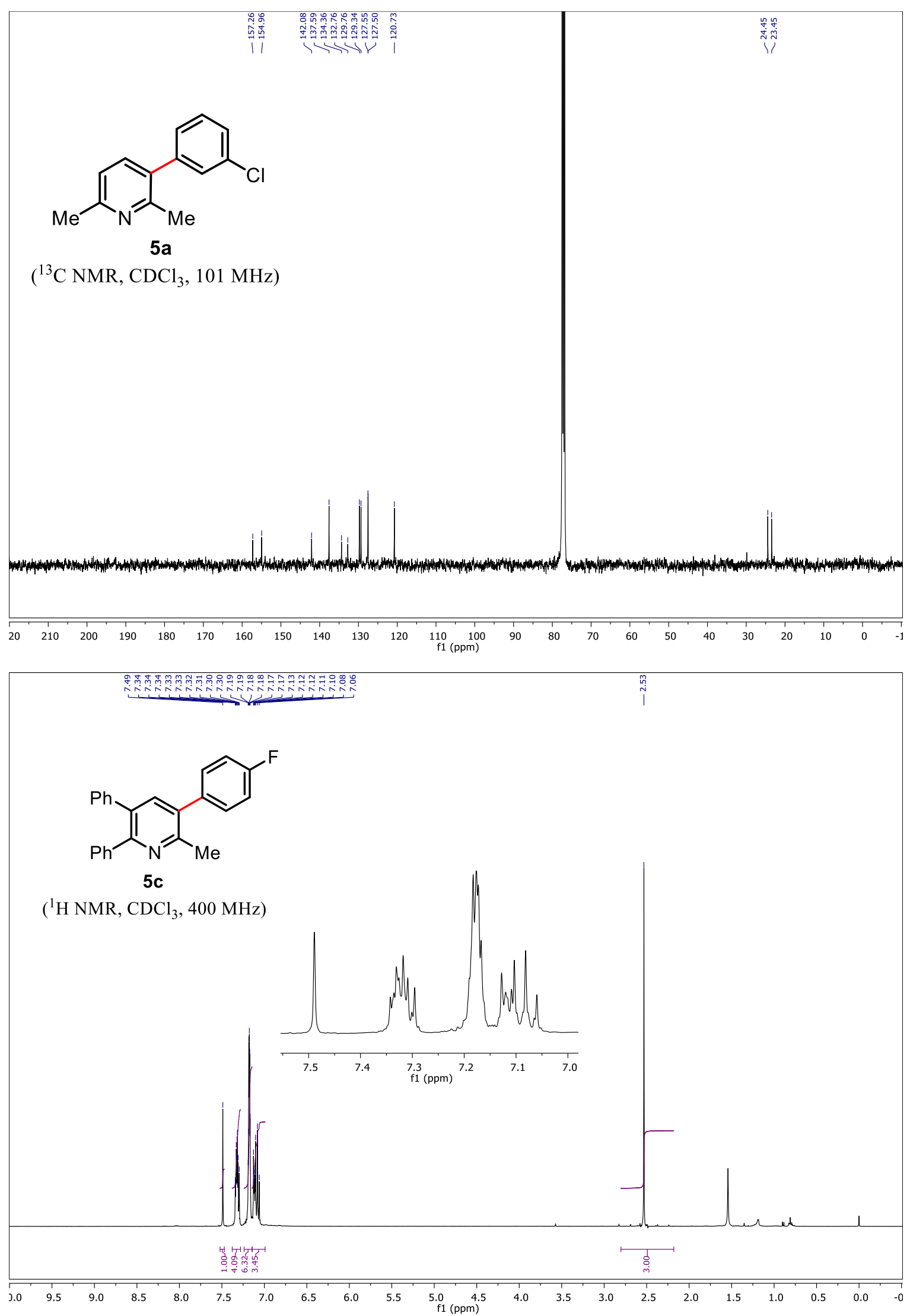

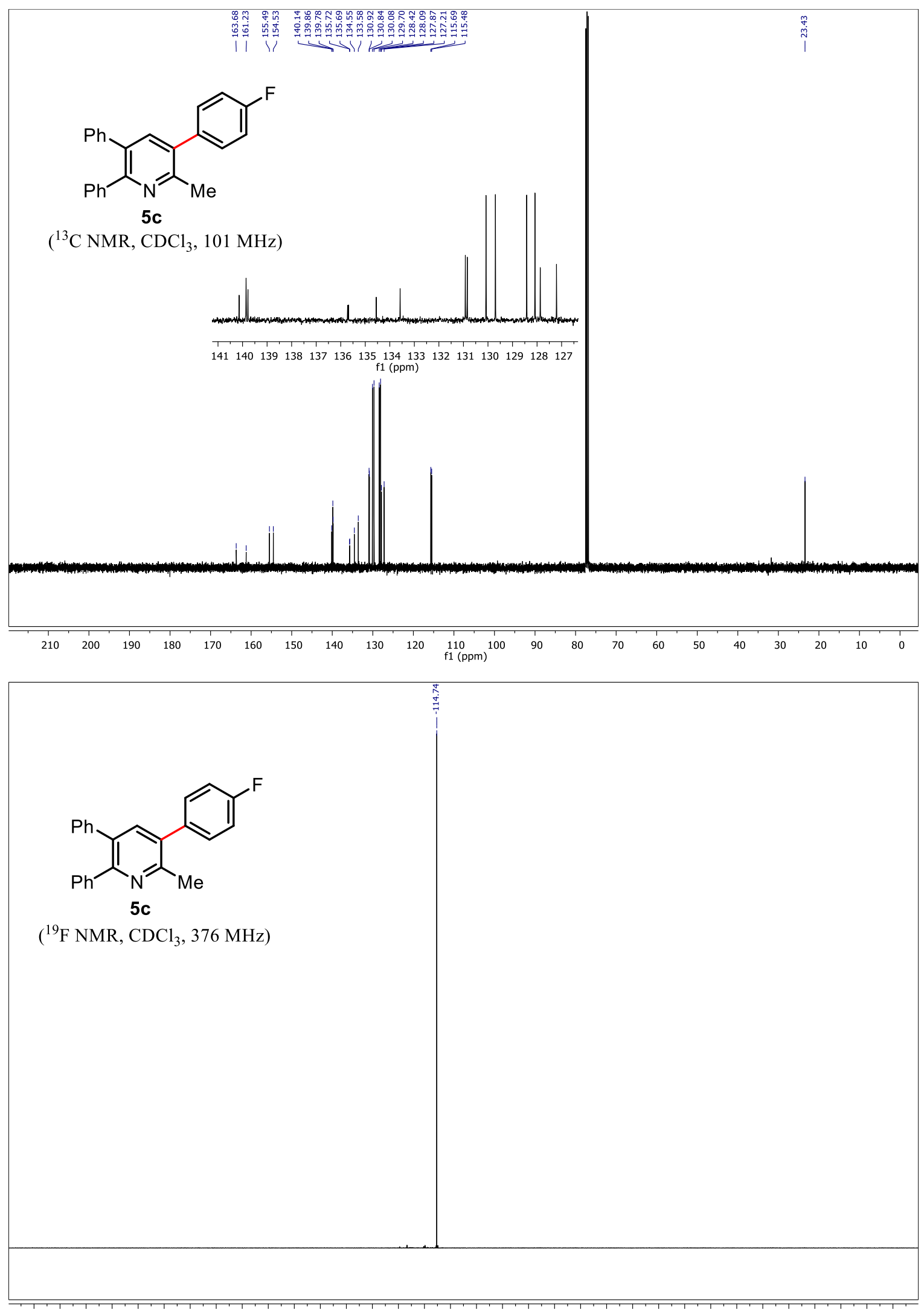

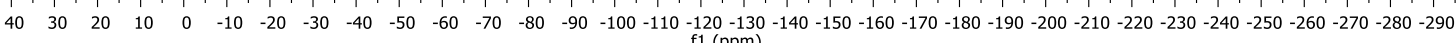


1D NOE NMR spectrum of 5c, $500 \mathrm{MHz}$, benzene- $d 6$
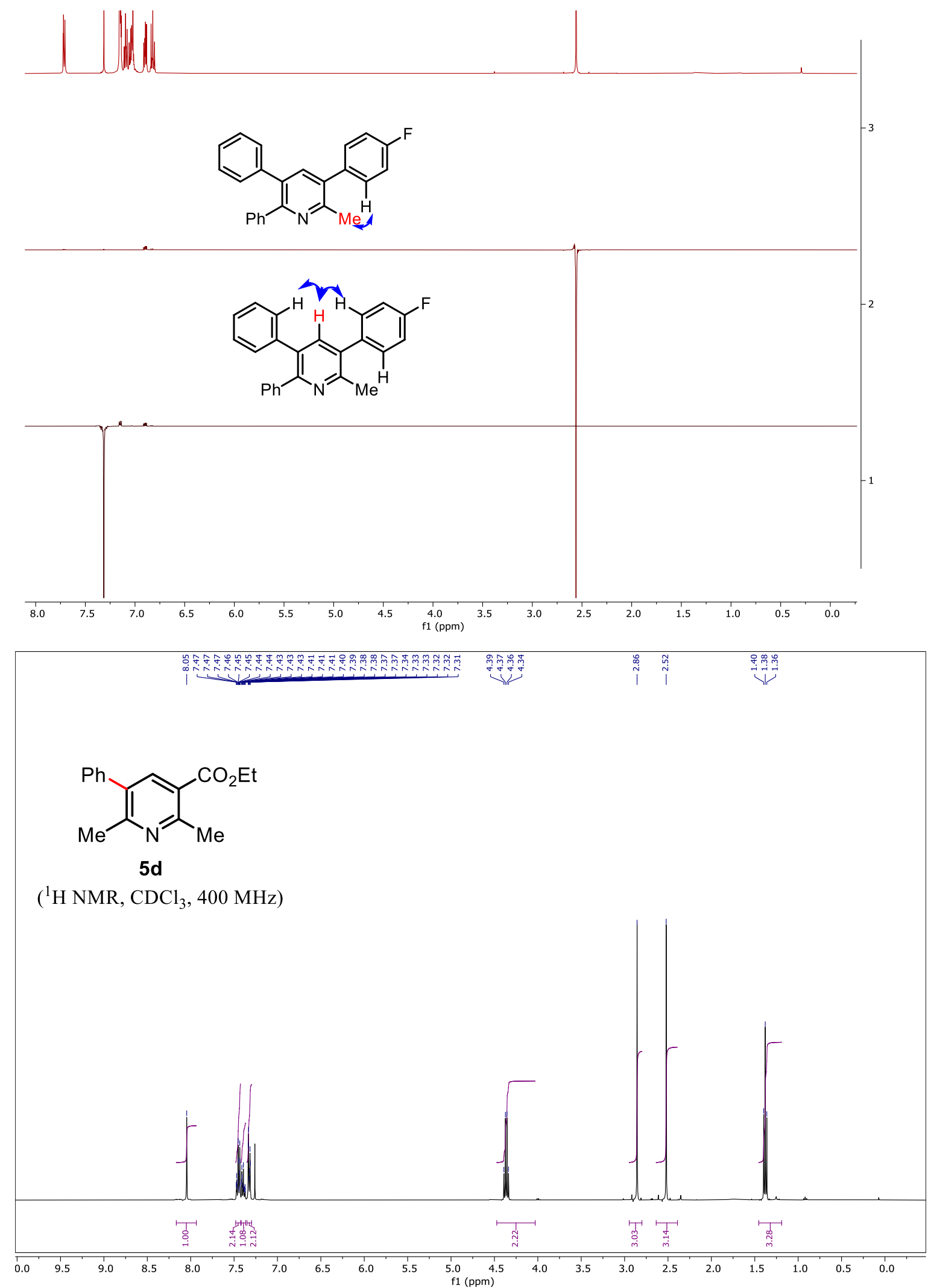


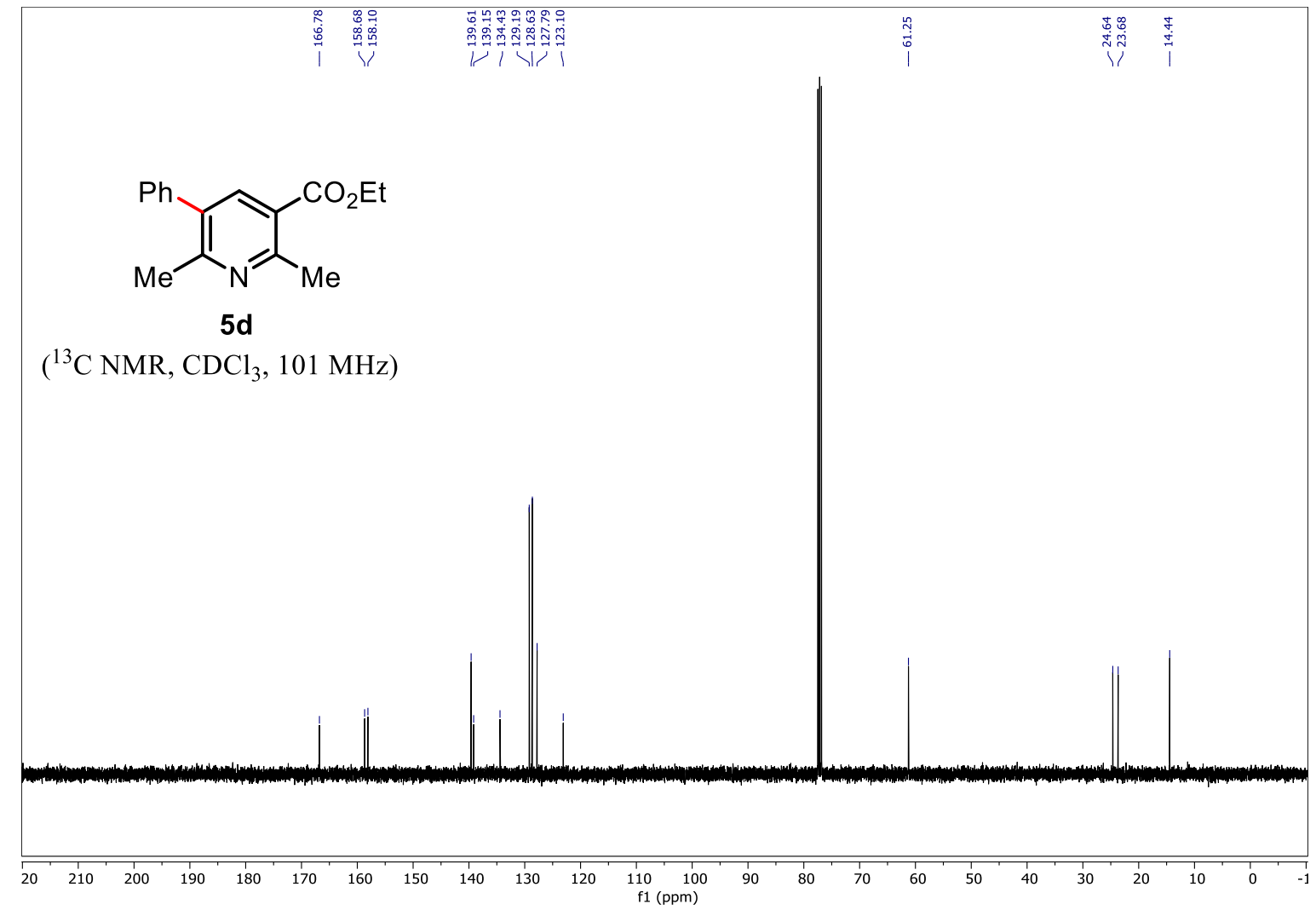

1D NOE NMR spectrum of $\mathbf{5 d}, 500 \mathrm{MHz}, \mathrm{CDCl}_{3}$
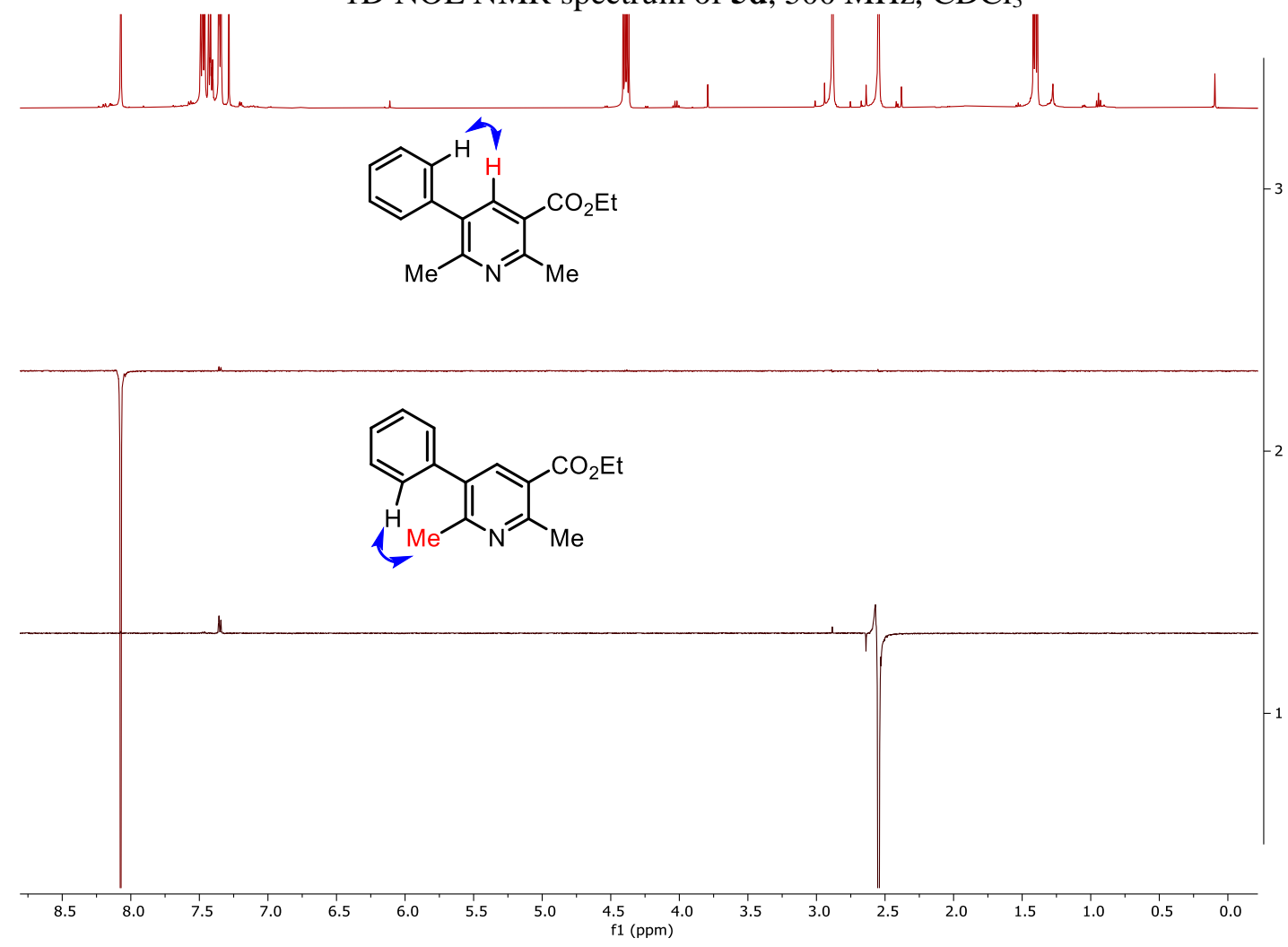

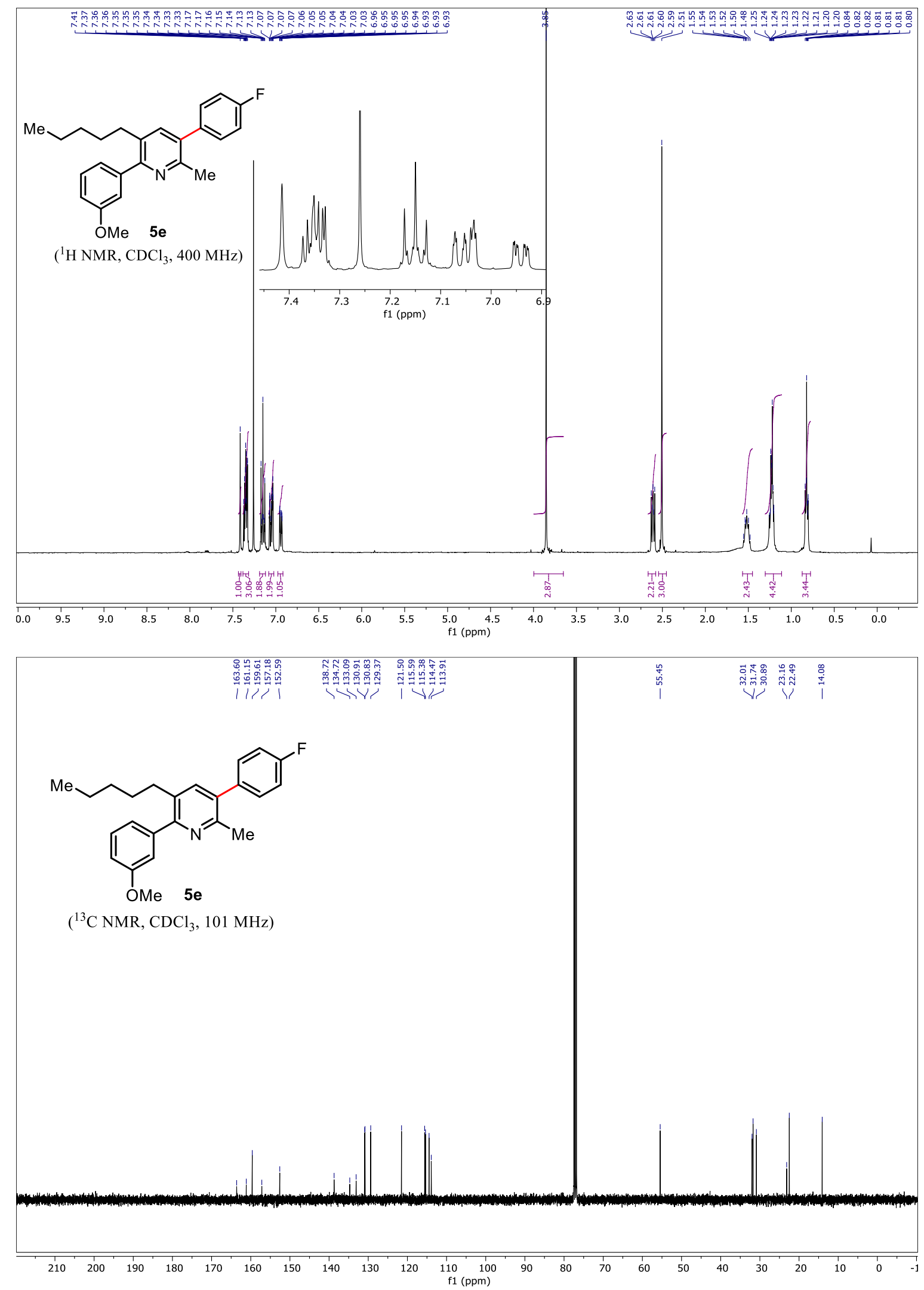


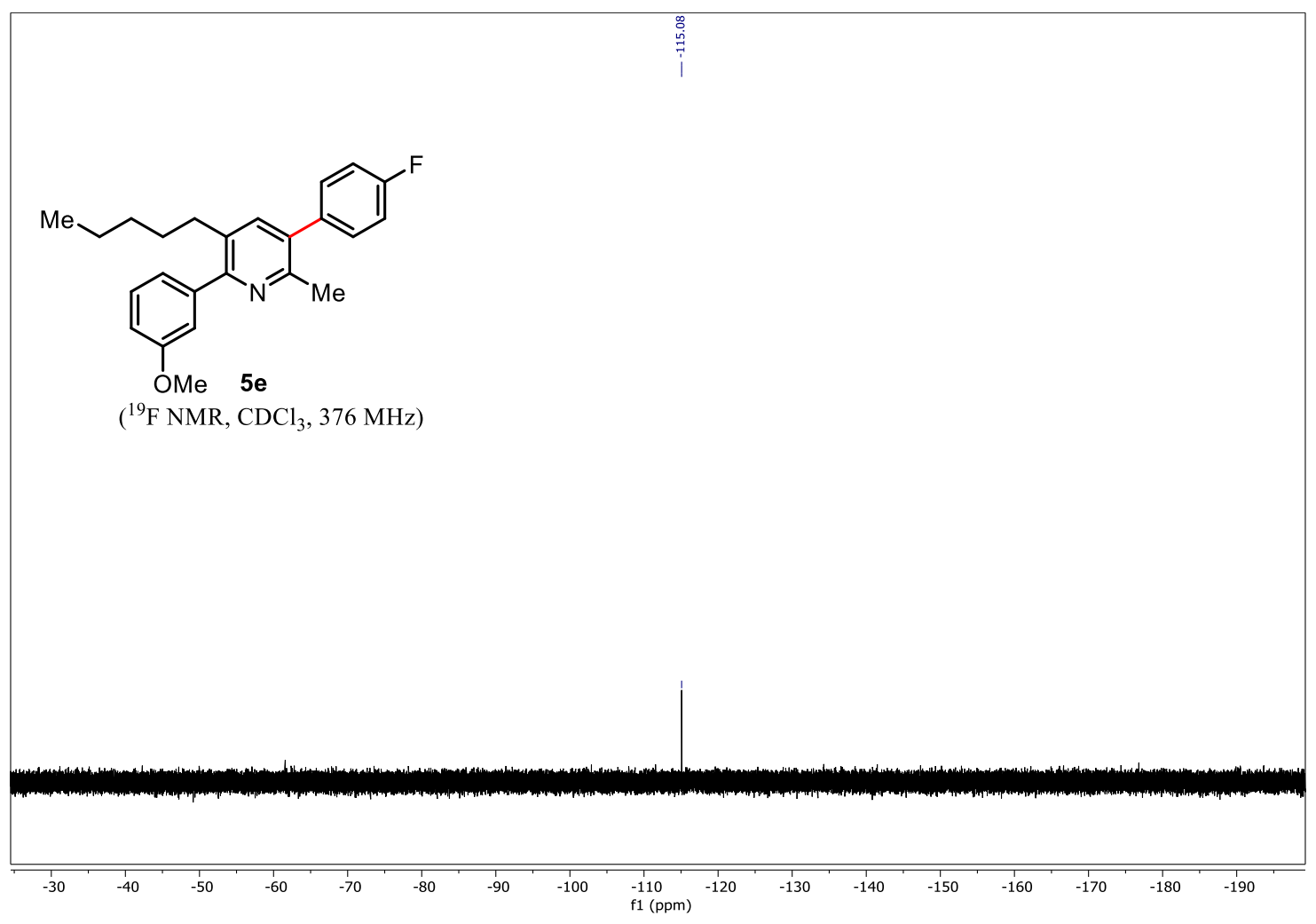

1D NOE NMR spectrum of $\mathbf{5 e}, 500 \mathrm{MHz}, \mathrm{CDCl}_{3}$

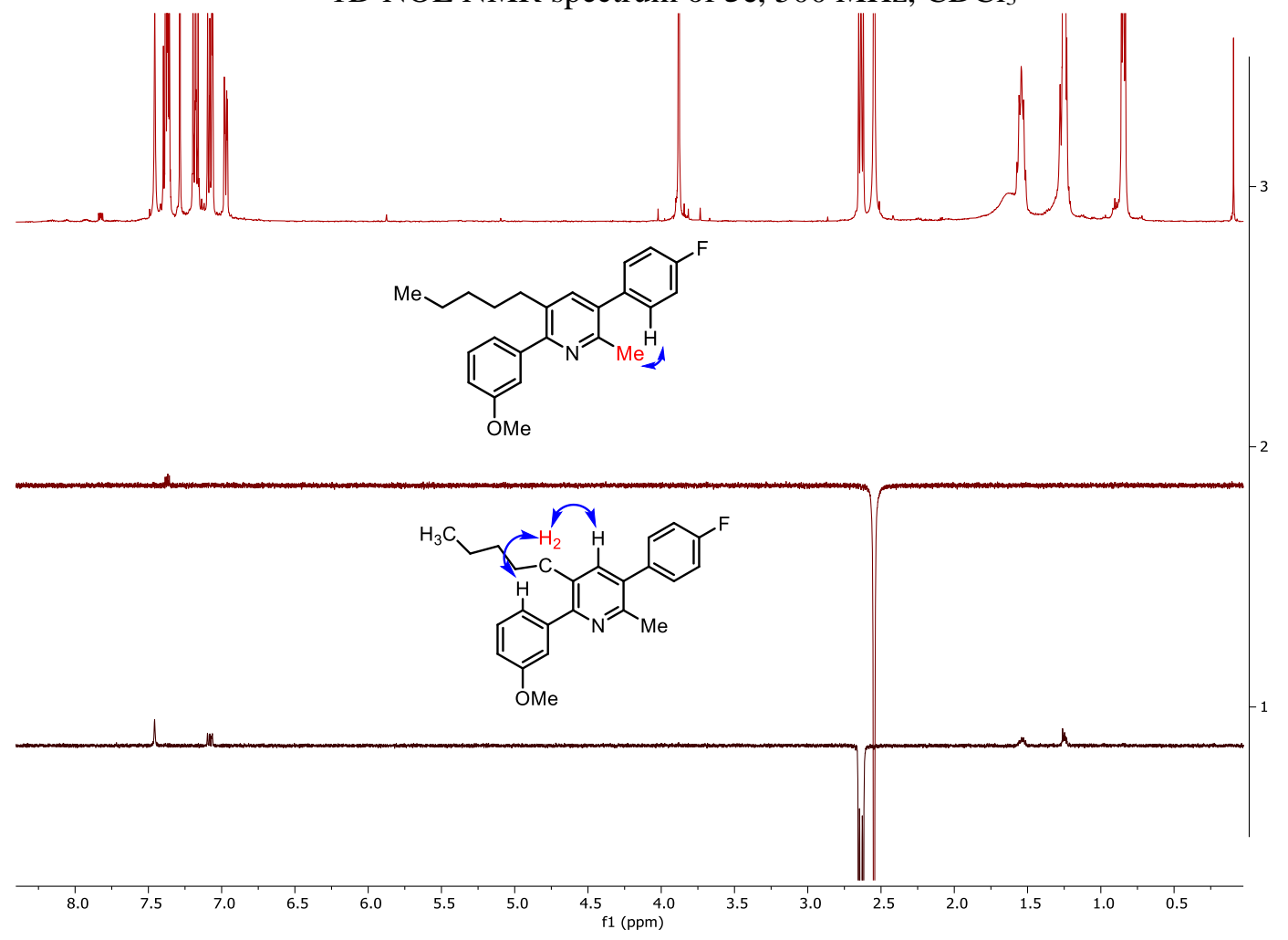



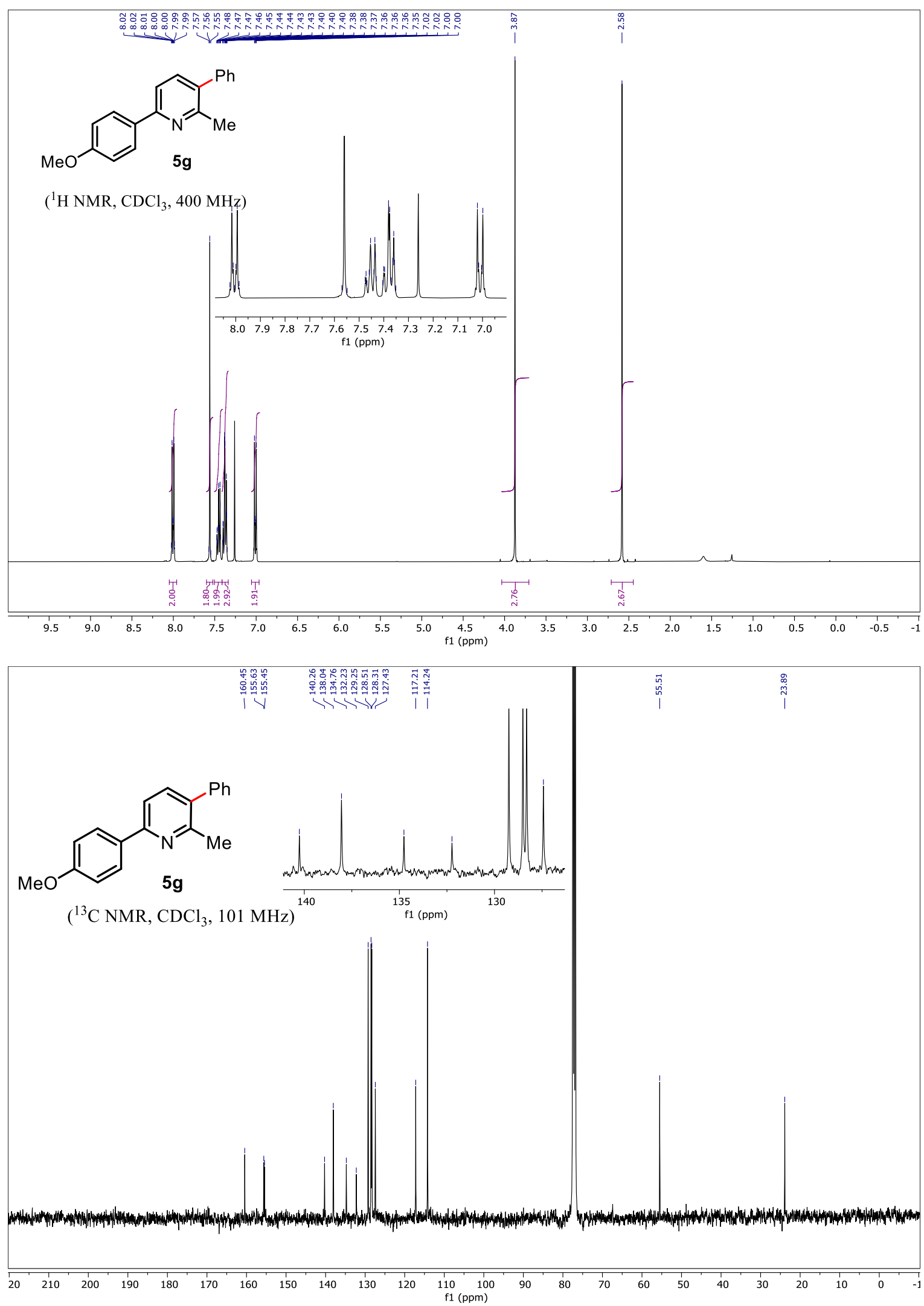
1D NOE NMR spectrum of $\mathbf{5 g}, 500 \mathrm{MHz}, \mathrm{C}_{6} \mathrm{D}_{6}$
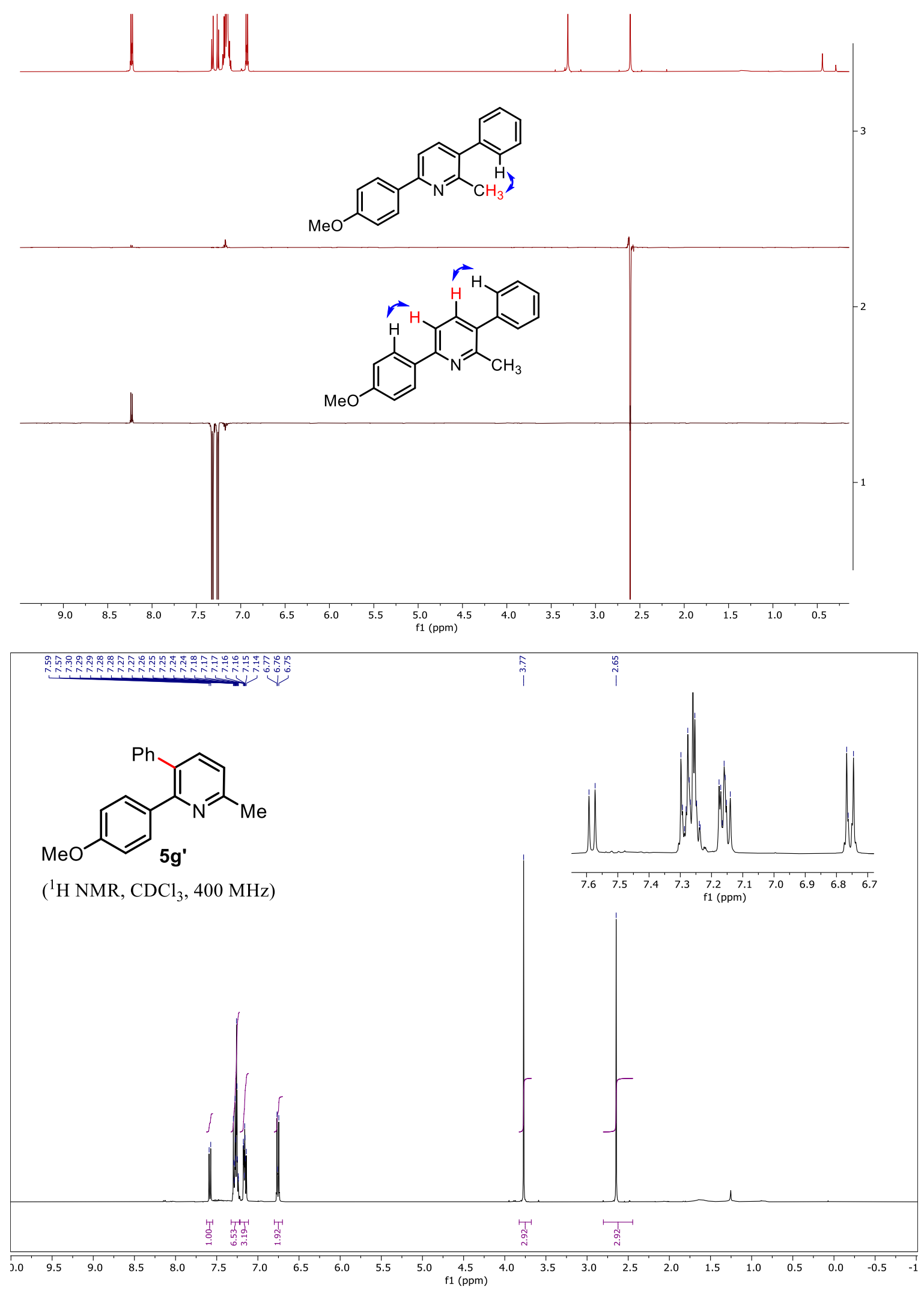


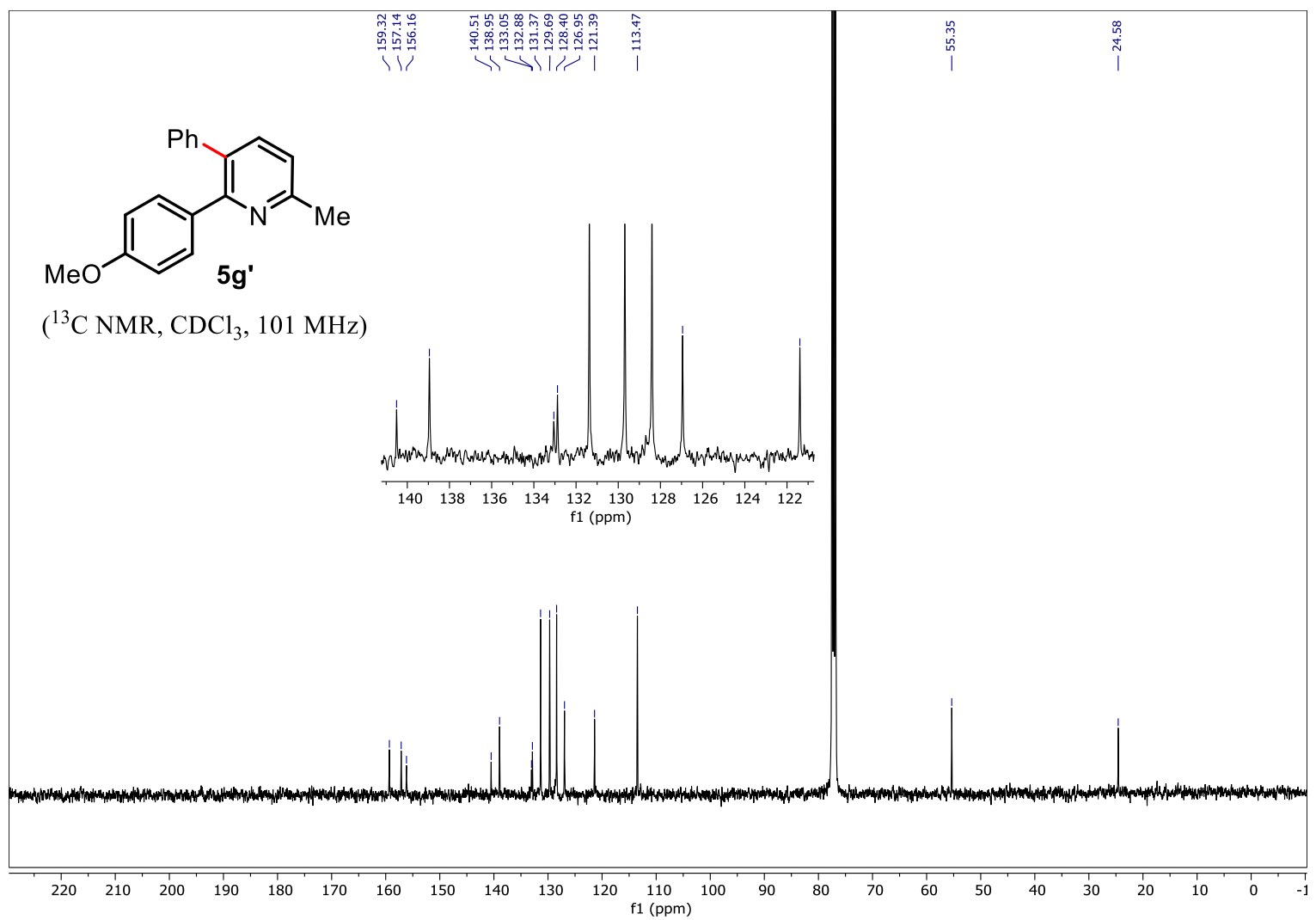

1D NOE NMR spectrum of $\mathbf{5} \mathbf{g}^{\prime}, 500 \mathrm{MHz}, \mathrm{C}_{6} \mathrm{D}_{6}$
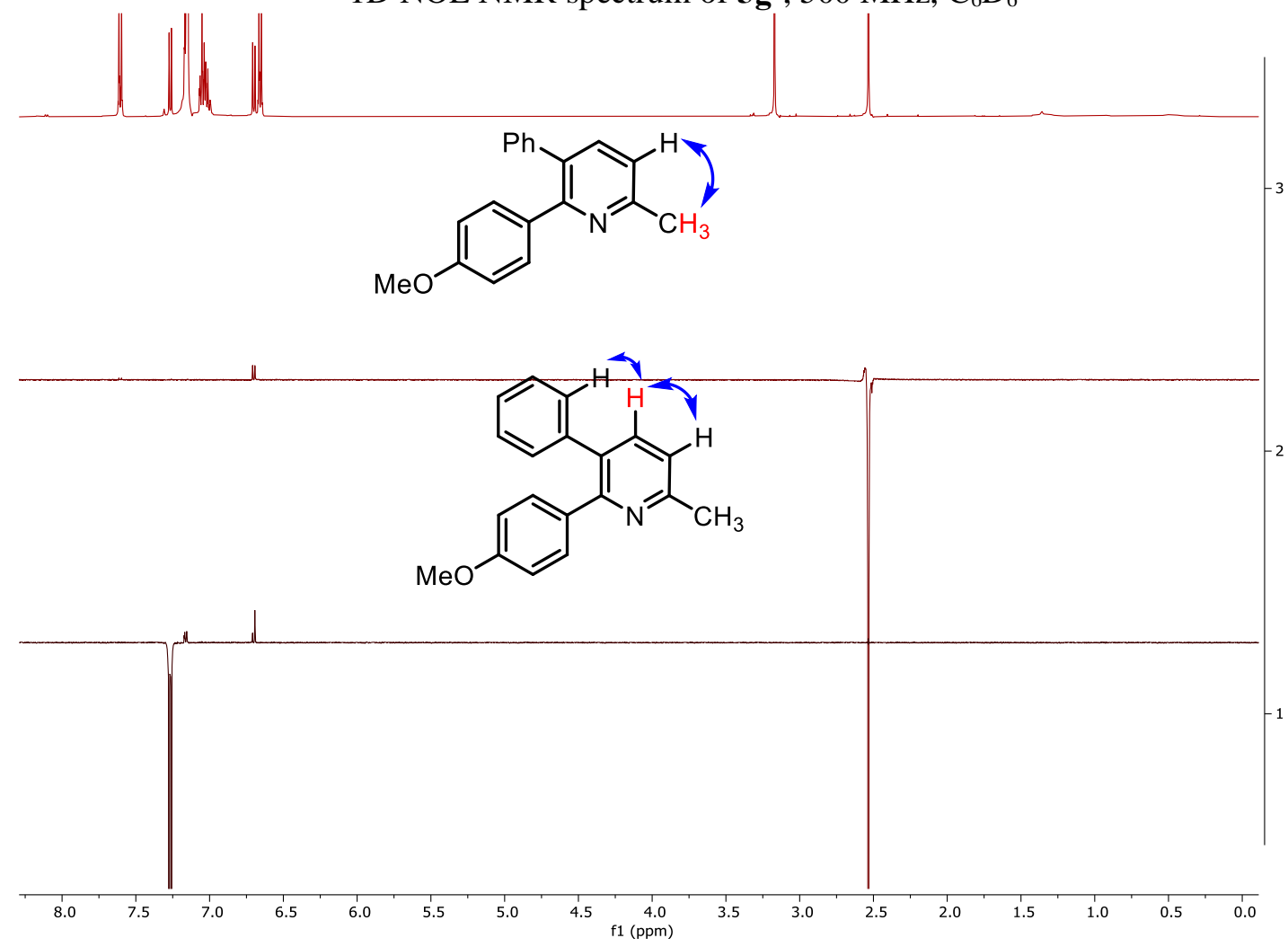

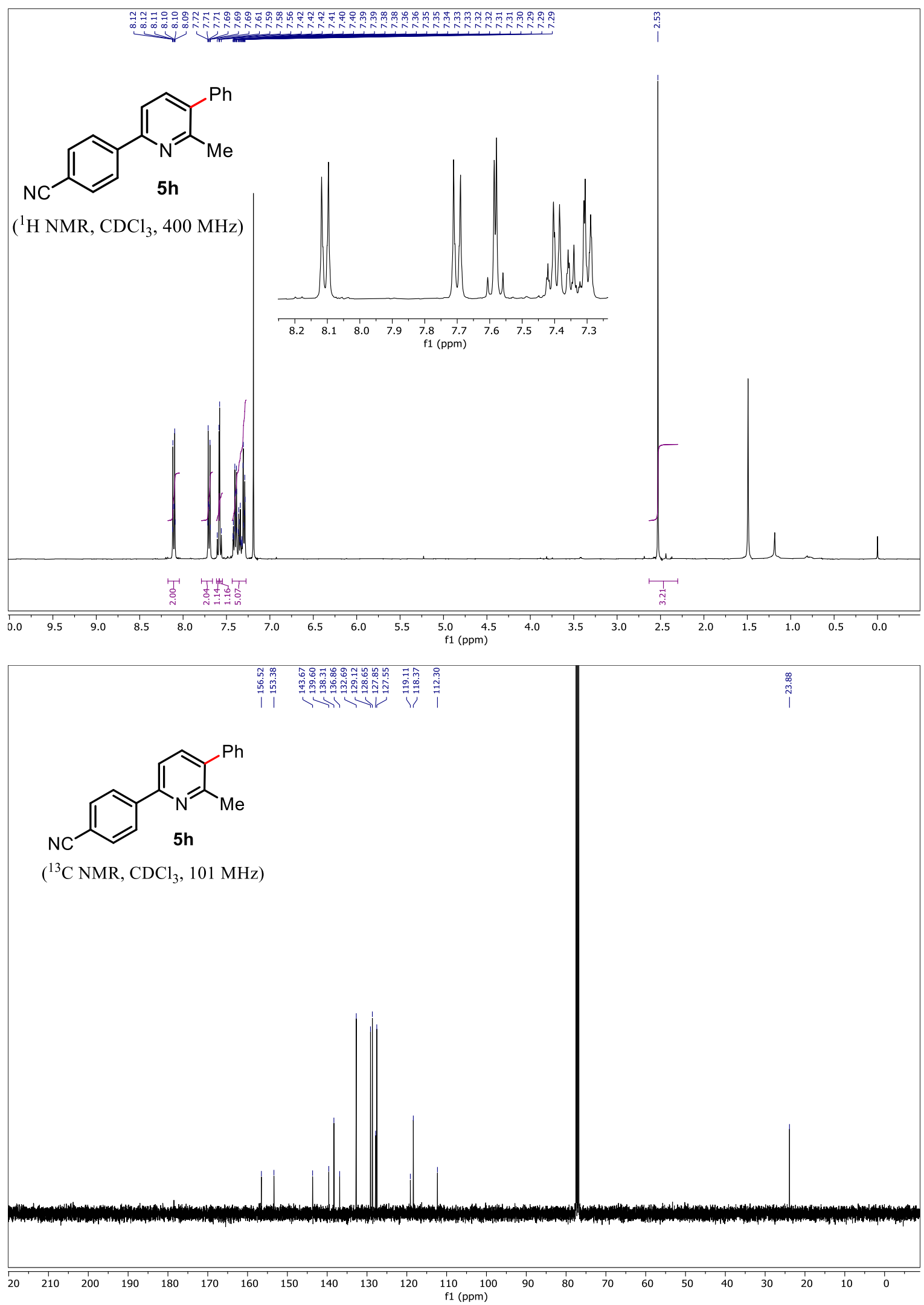
1D NOE NMR spectrum of $\mathbf{5 h}, 500 \mathbf{M H z}, \mathrm{CDCl}_{3}$
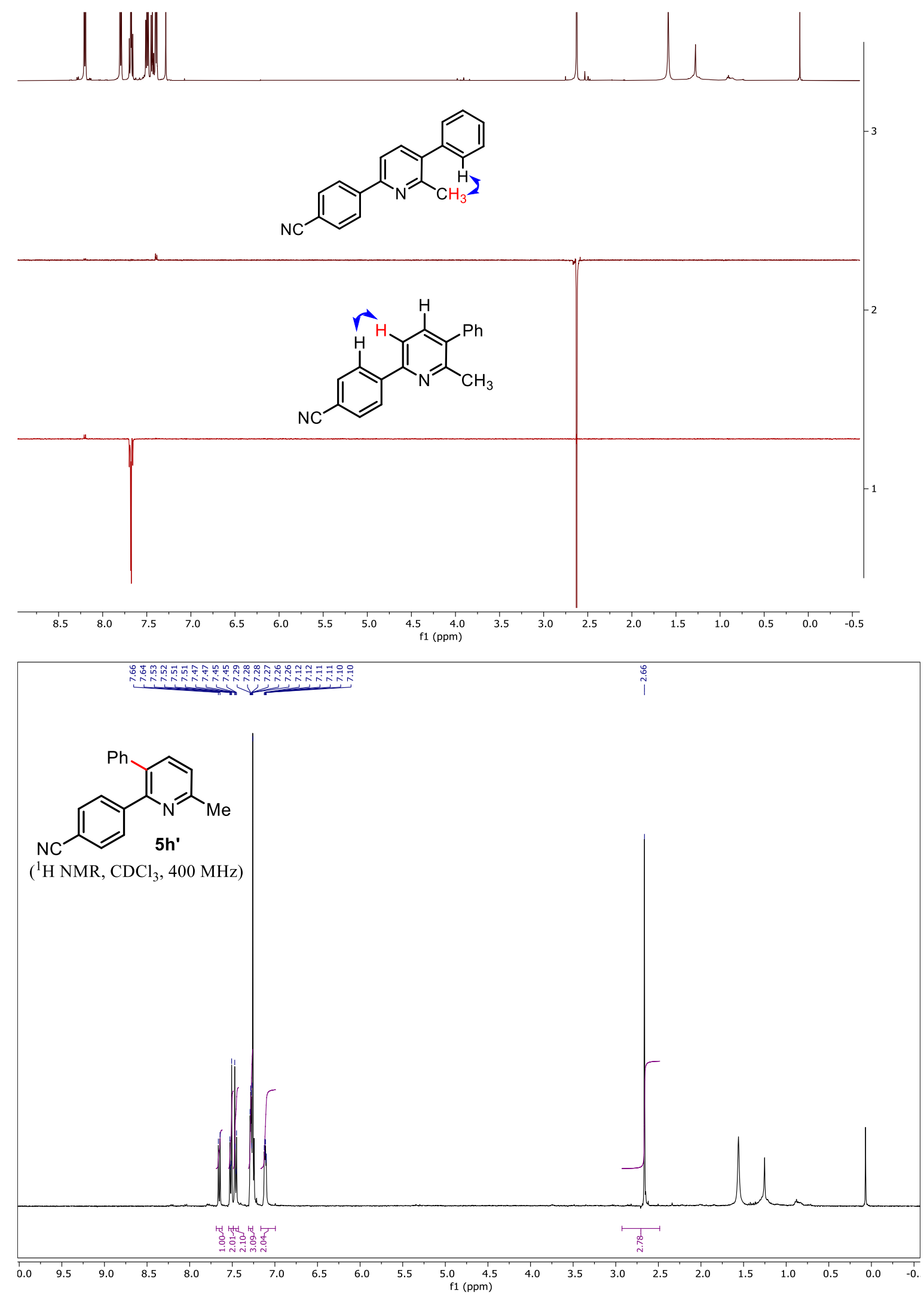

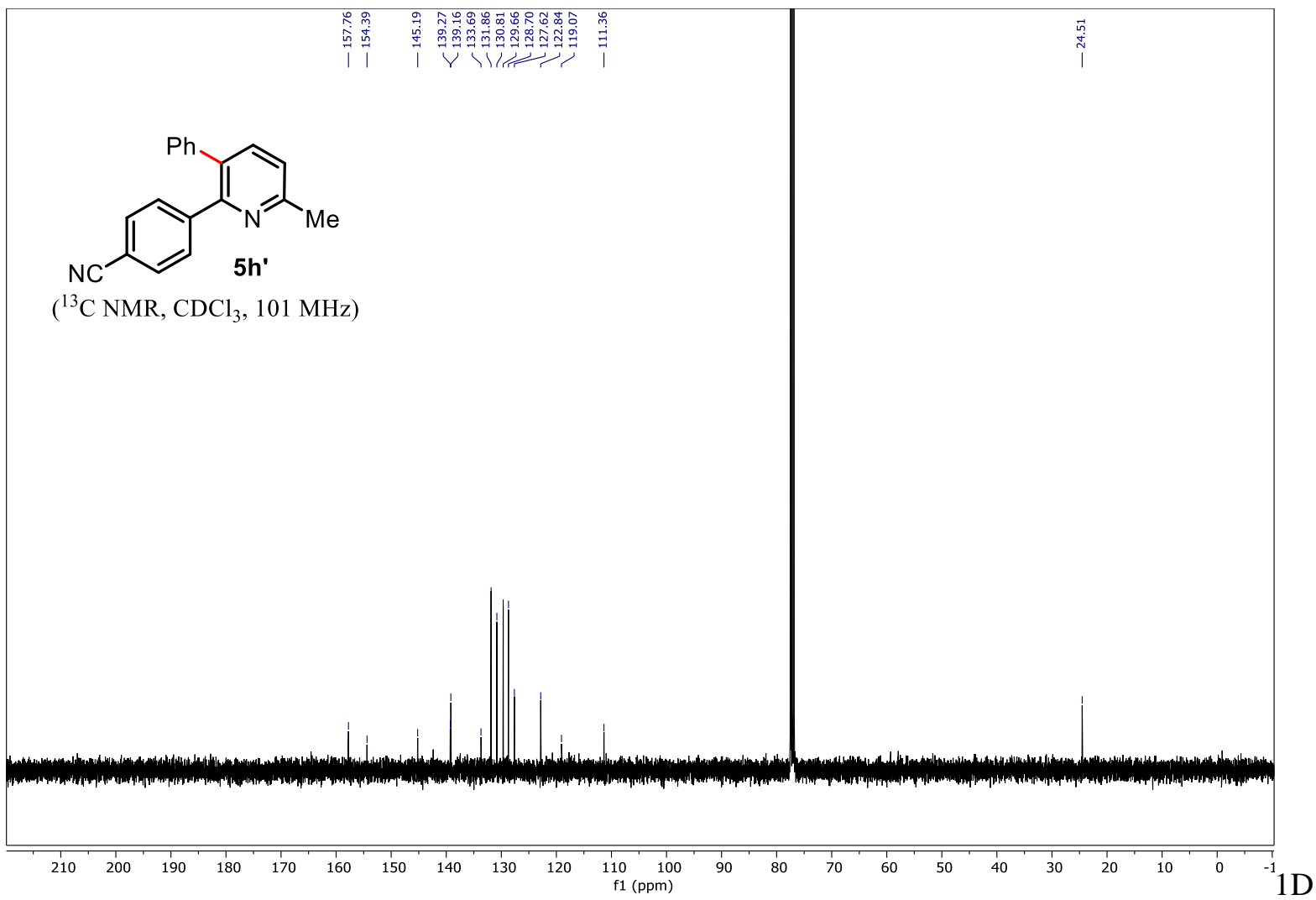

NOE NMR spectrum of $\mathbf{5 h}$ ', $500 \mathrm{MHz}, \mathrm{CDCl}_{3}$
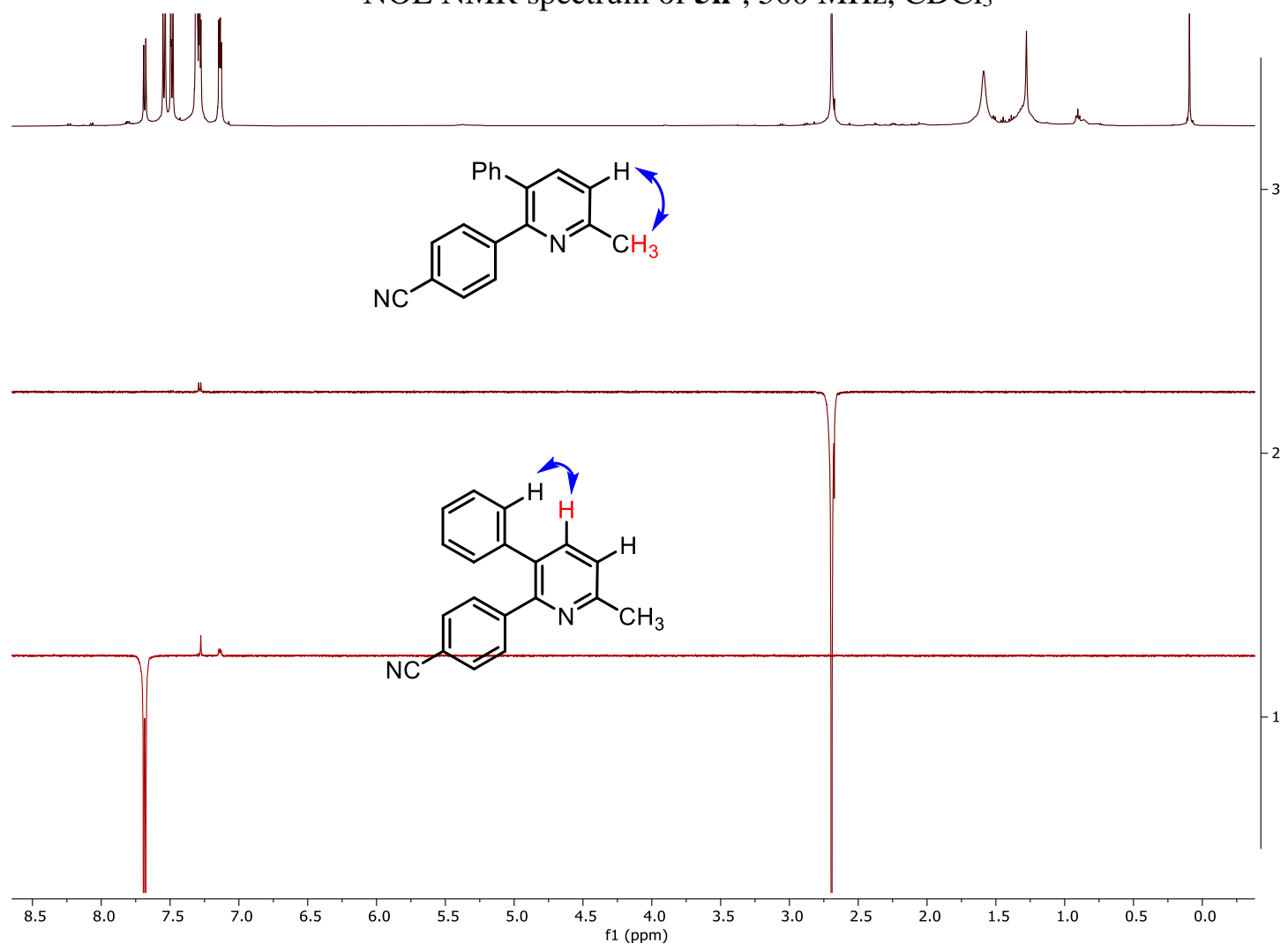

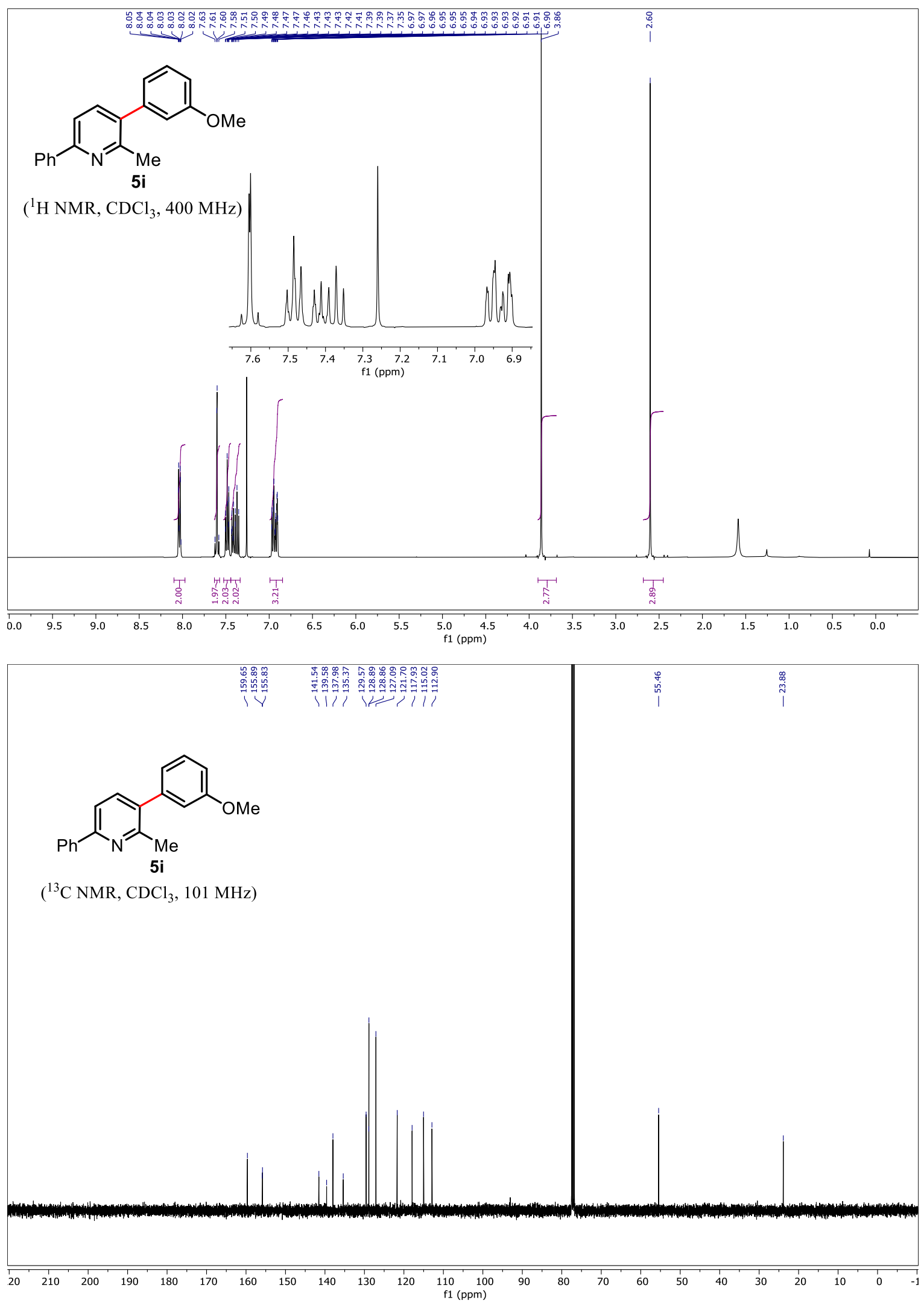
1D NOE NMR spectrum of $\mathbf{5 i}, 500 \mathrm{MHz}, \mathrm{CDCl}_{3}$
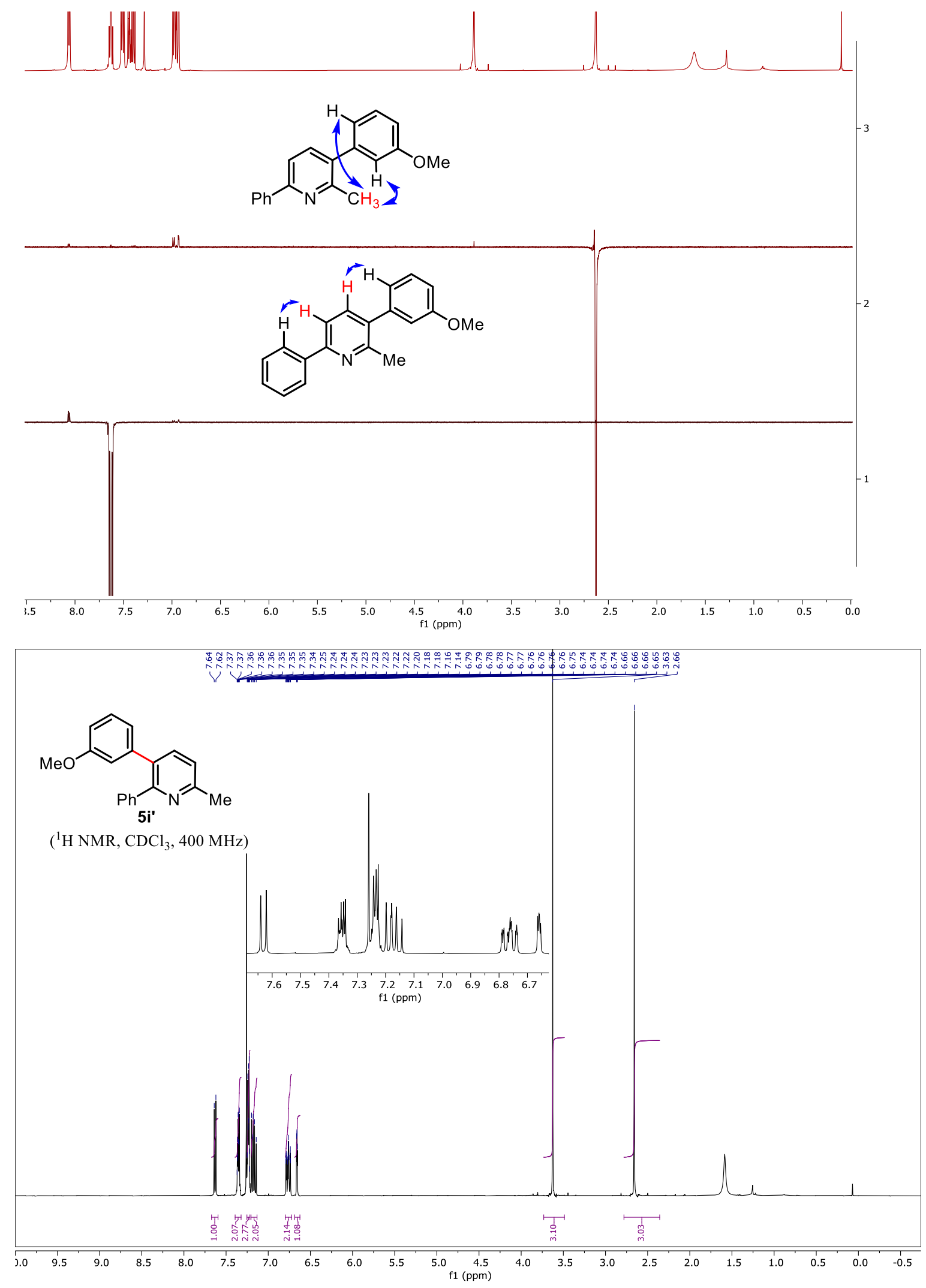


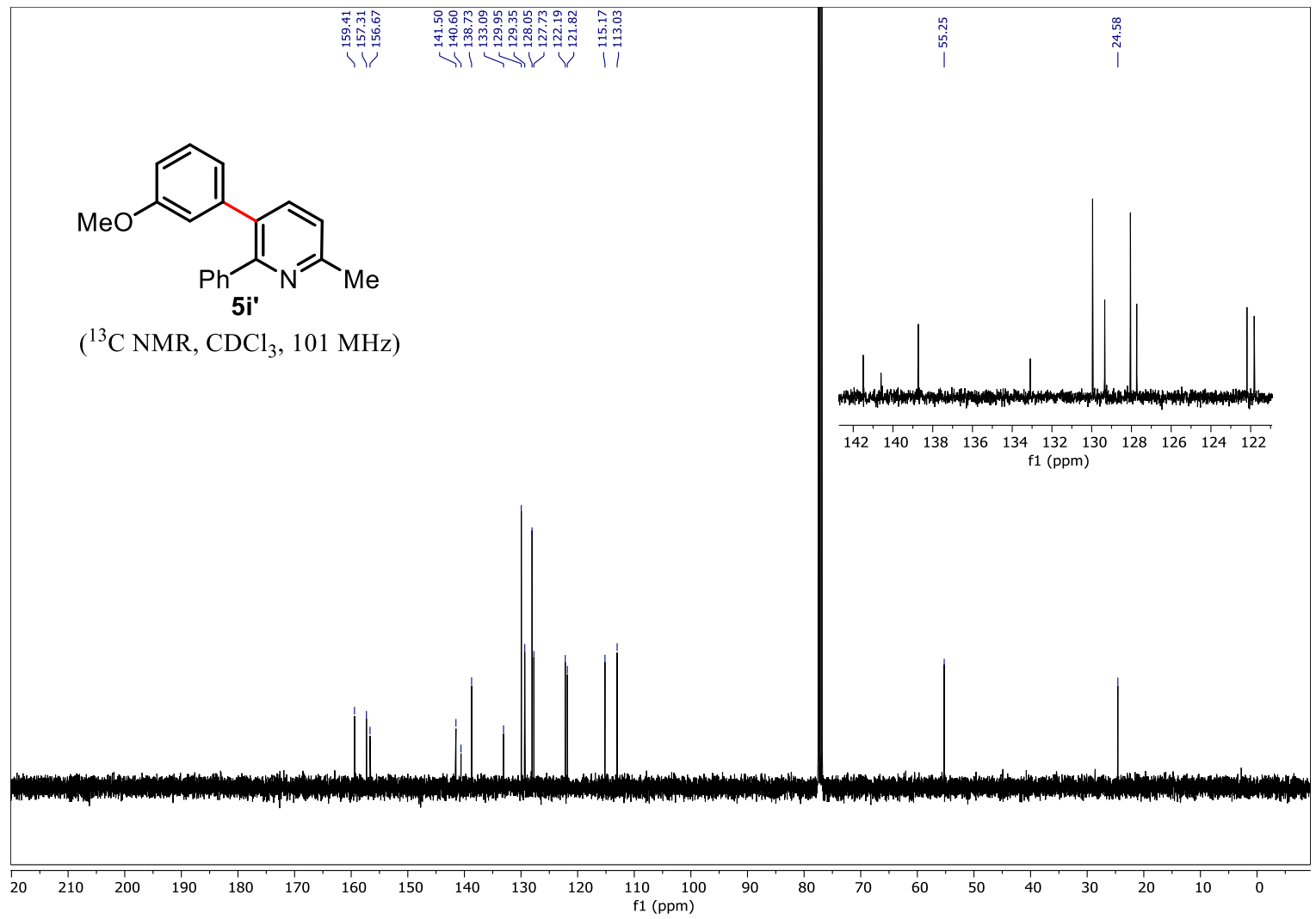

1D NOE NMR spectrum of $\mathbf{5 i}, 500 \mathrm{MHz}, \mathrm{CDCl}_{3}$
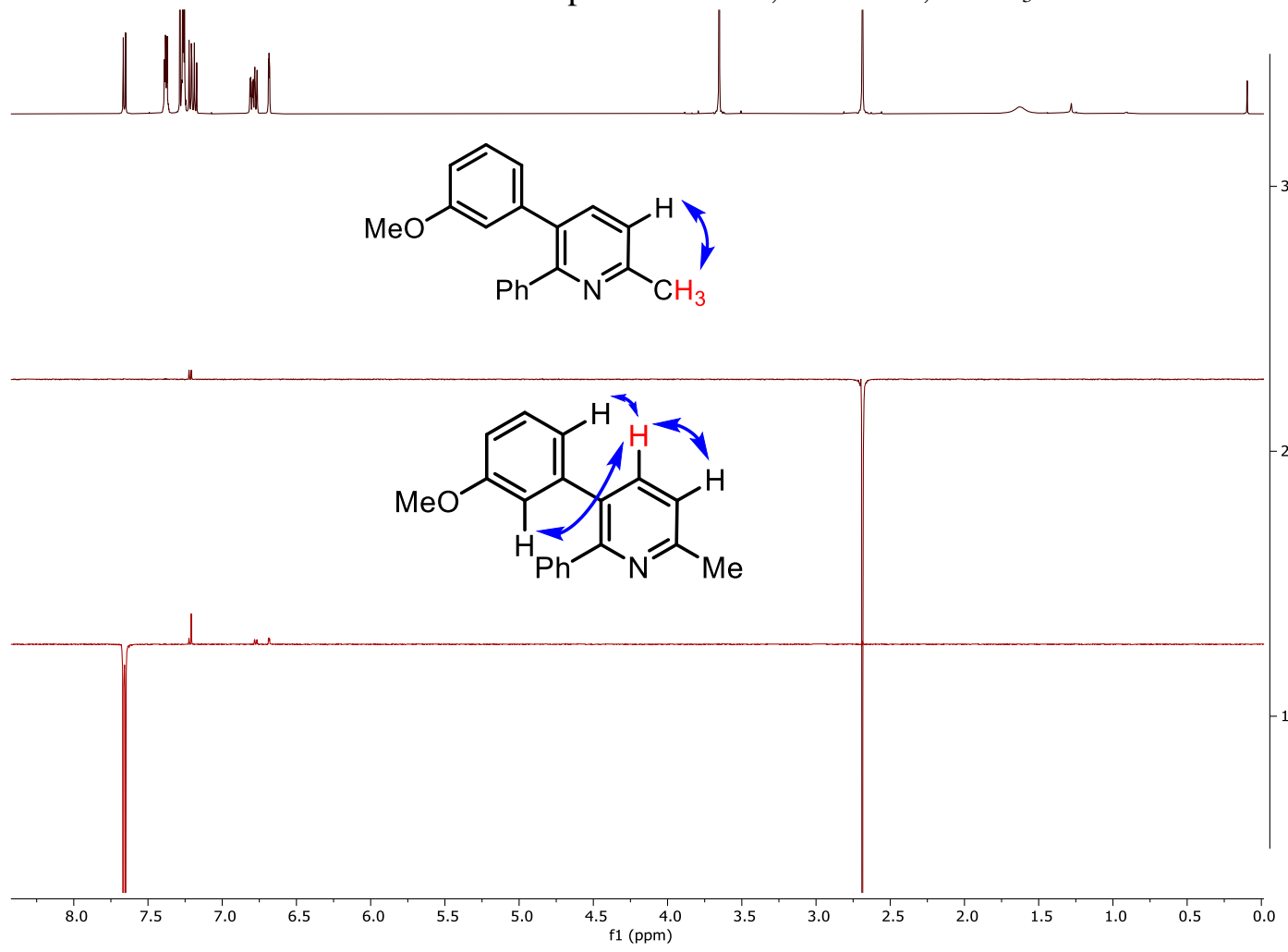

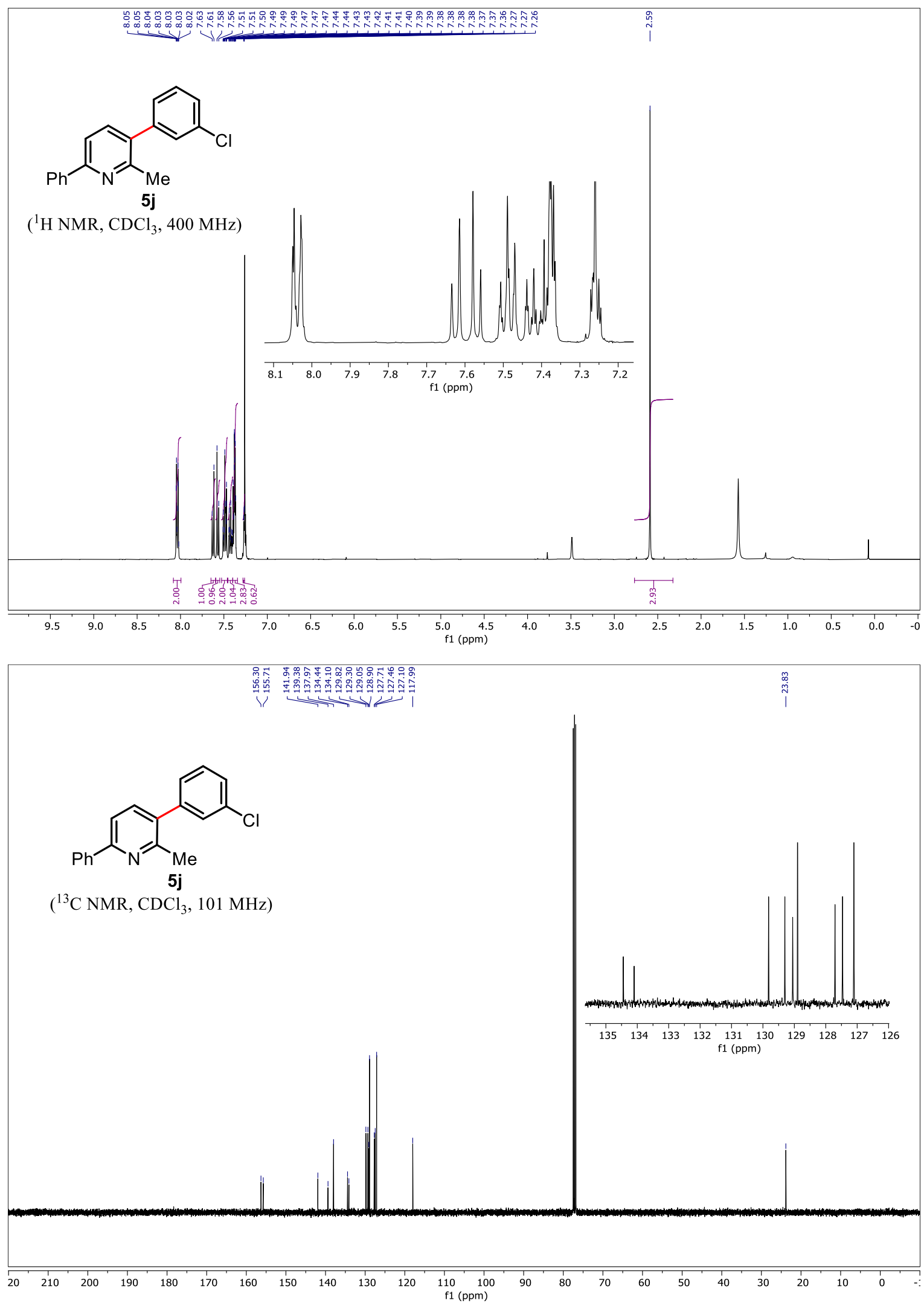
1D NOE NMR spectrum of $\mathbf{5 j}, 500 \mathrm{MHz}, \mathrm{CDCl}_{3}$
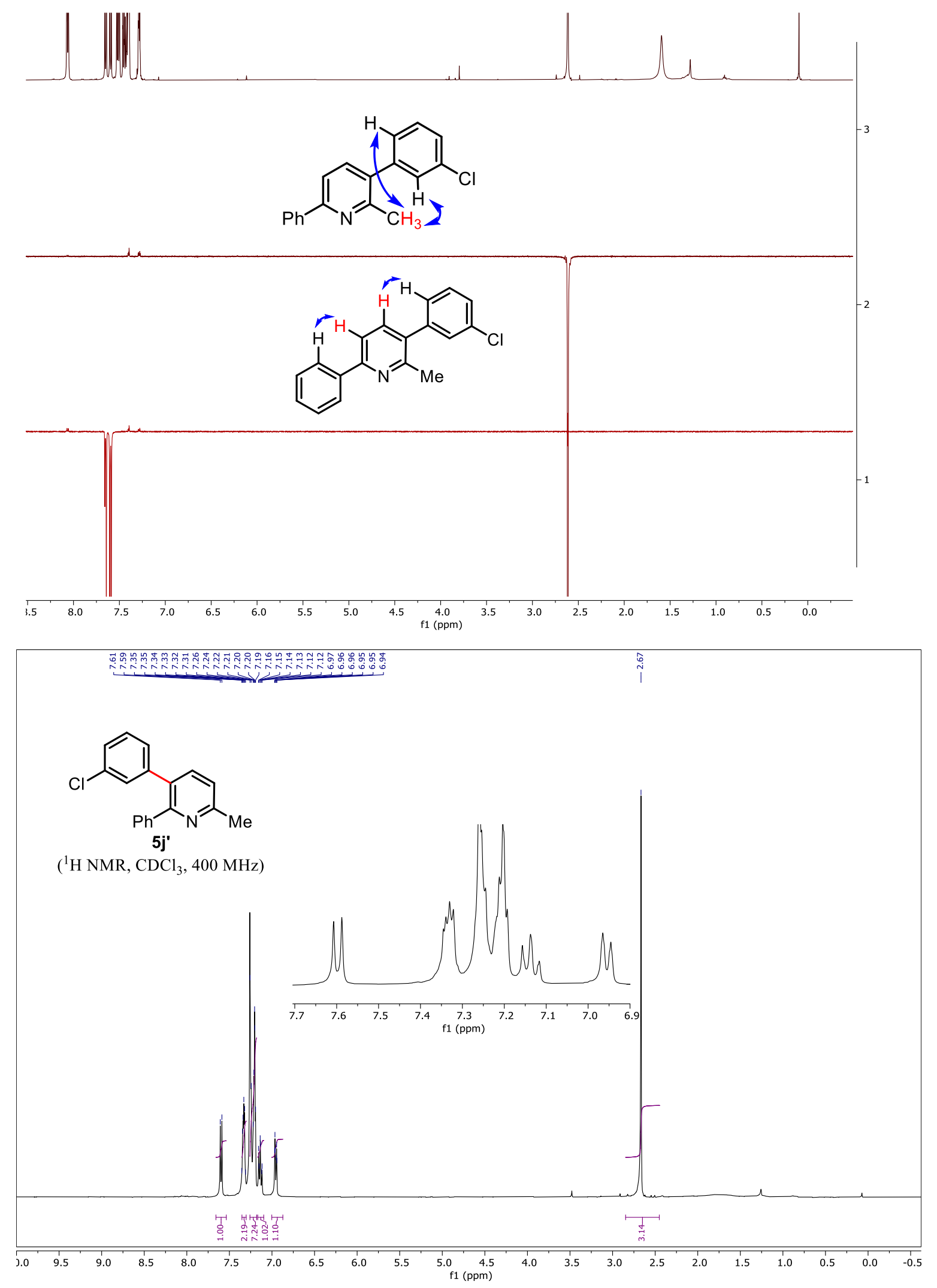


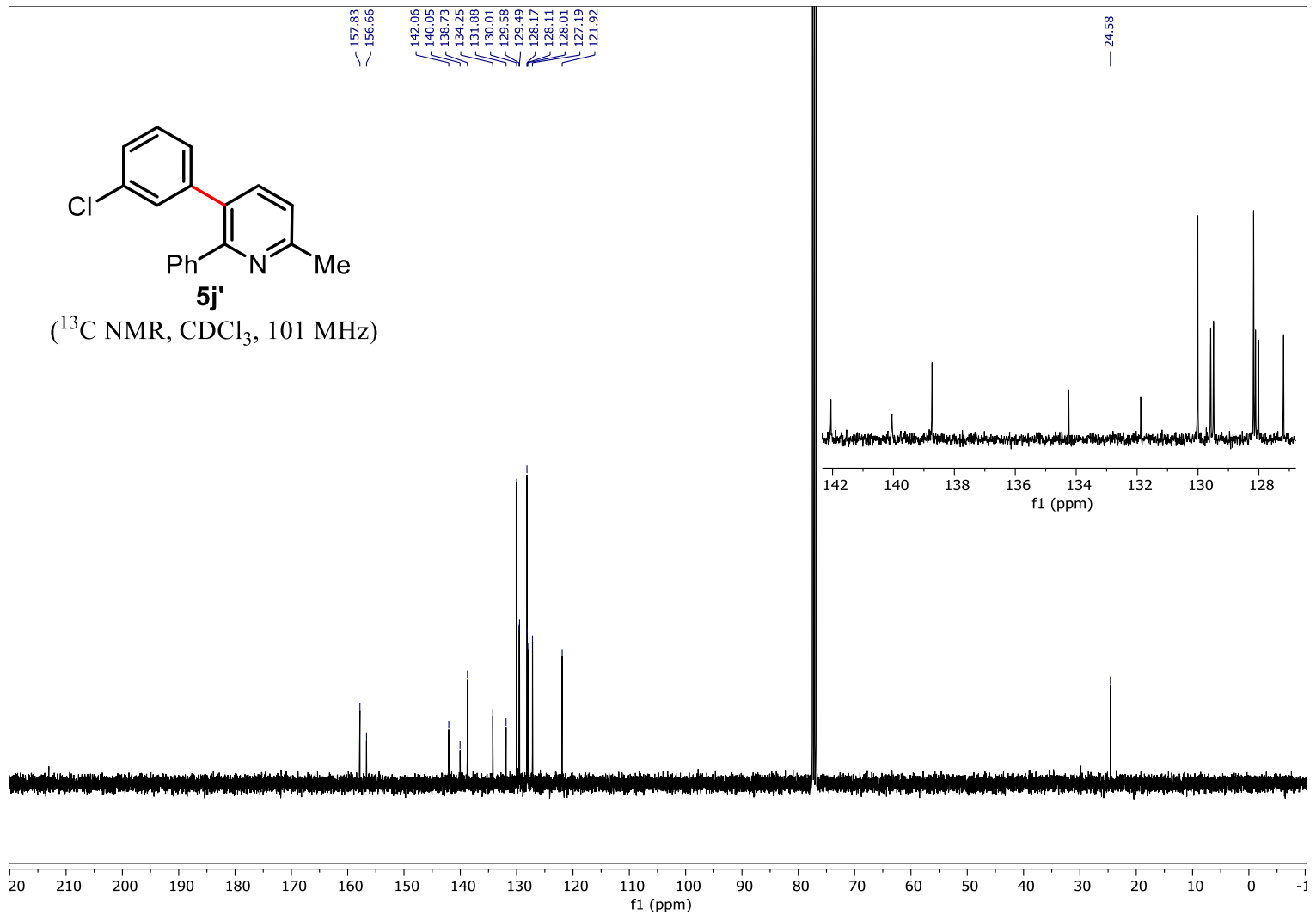

1D NOE NMR spectrum of 5j', $500 \mathrm{MHz}, \mathrm{C}_{6} \mathrm{D}_{6}$
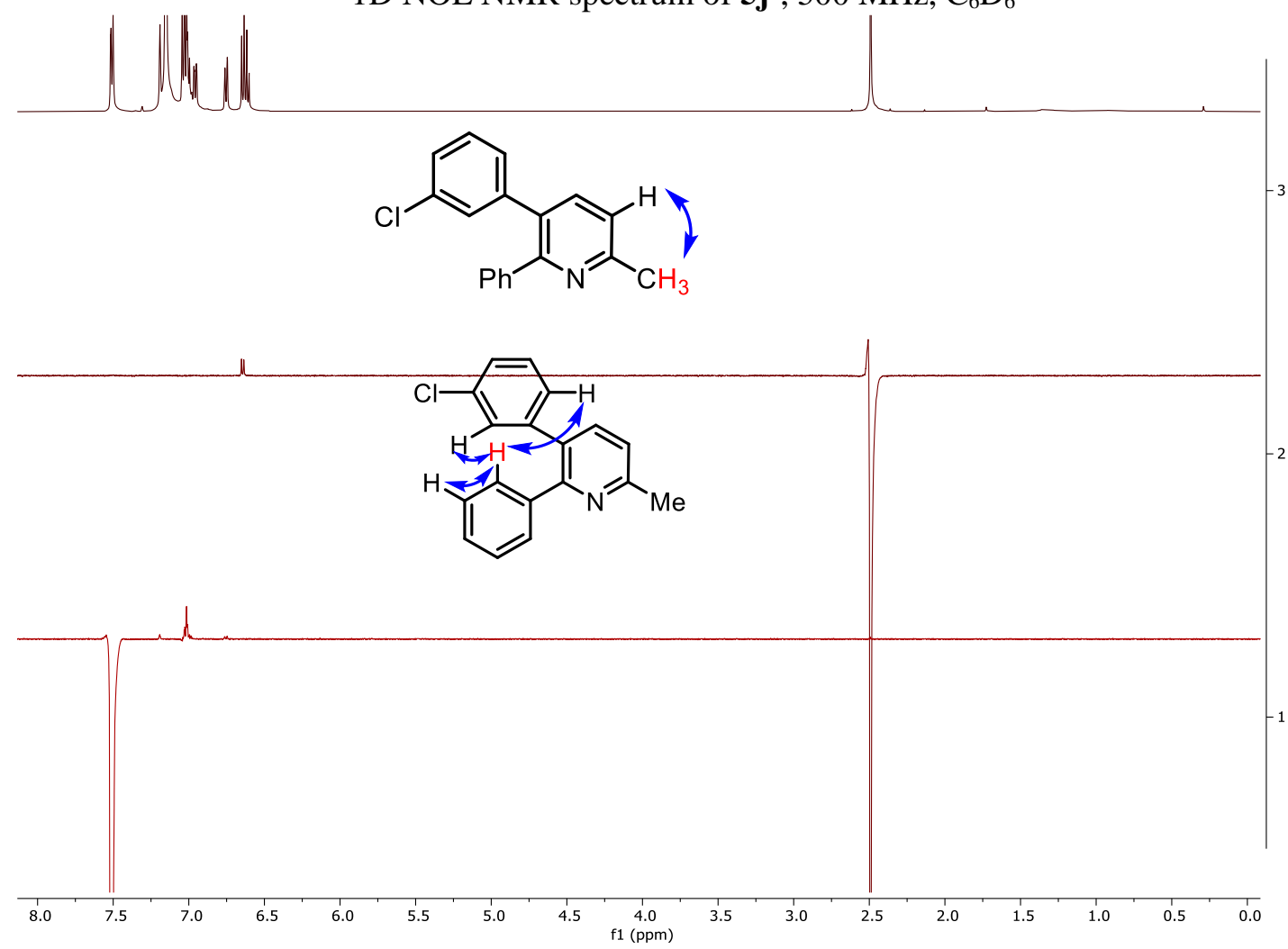

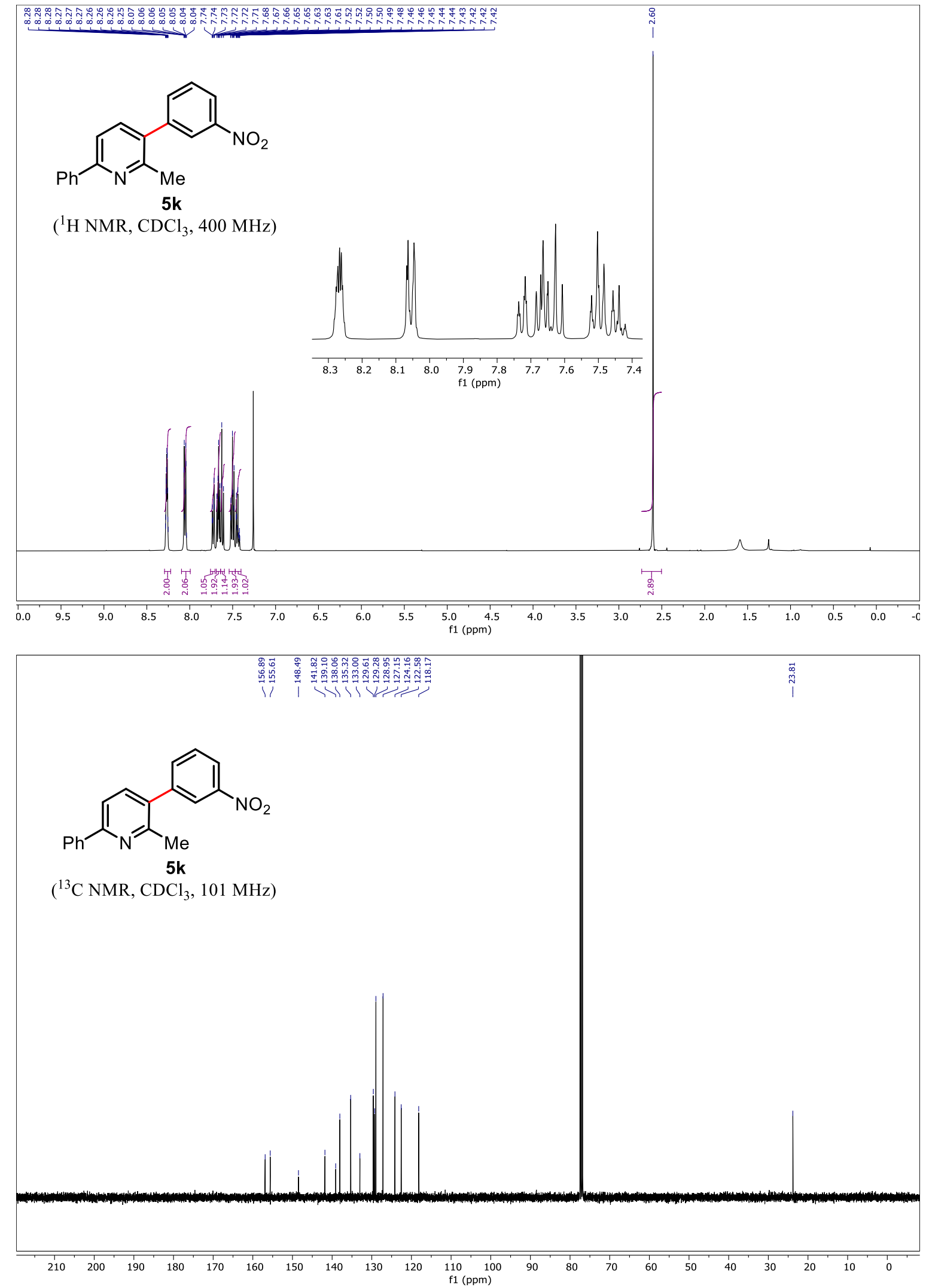
1D NOE NMR spectrum of $\mathbf{5 k}, 500 \mathrm{MHz}, \mathrm{C}_{6} \mathrm{D}_{6}$
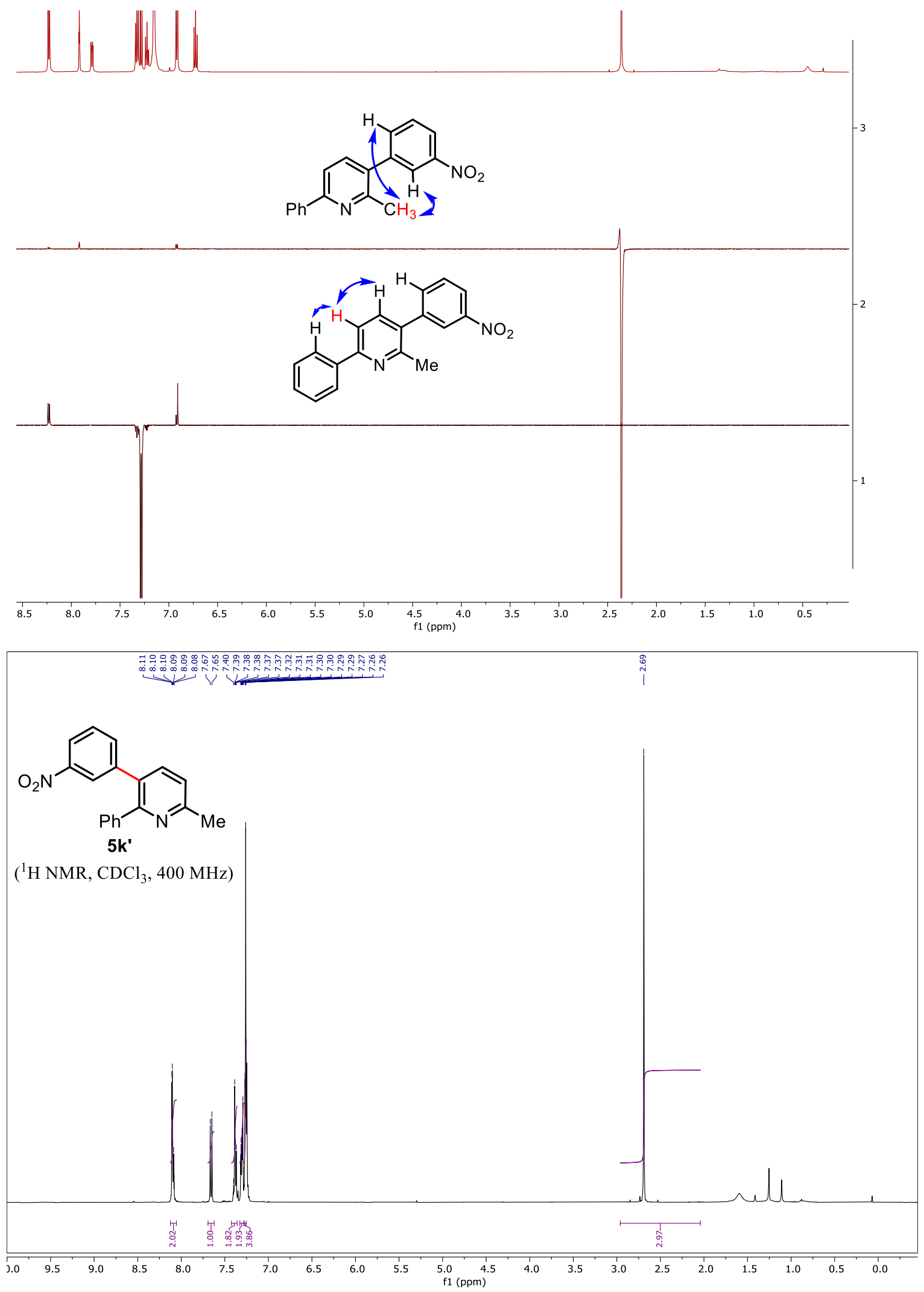


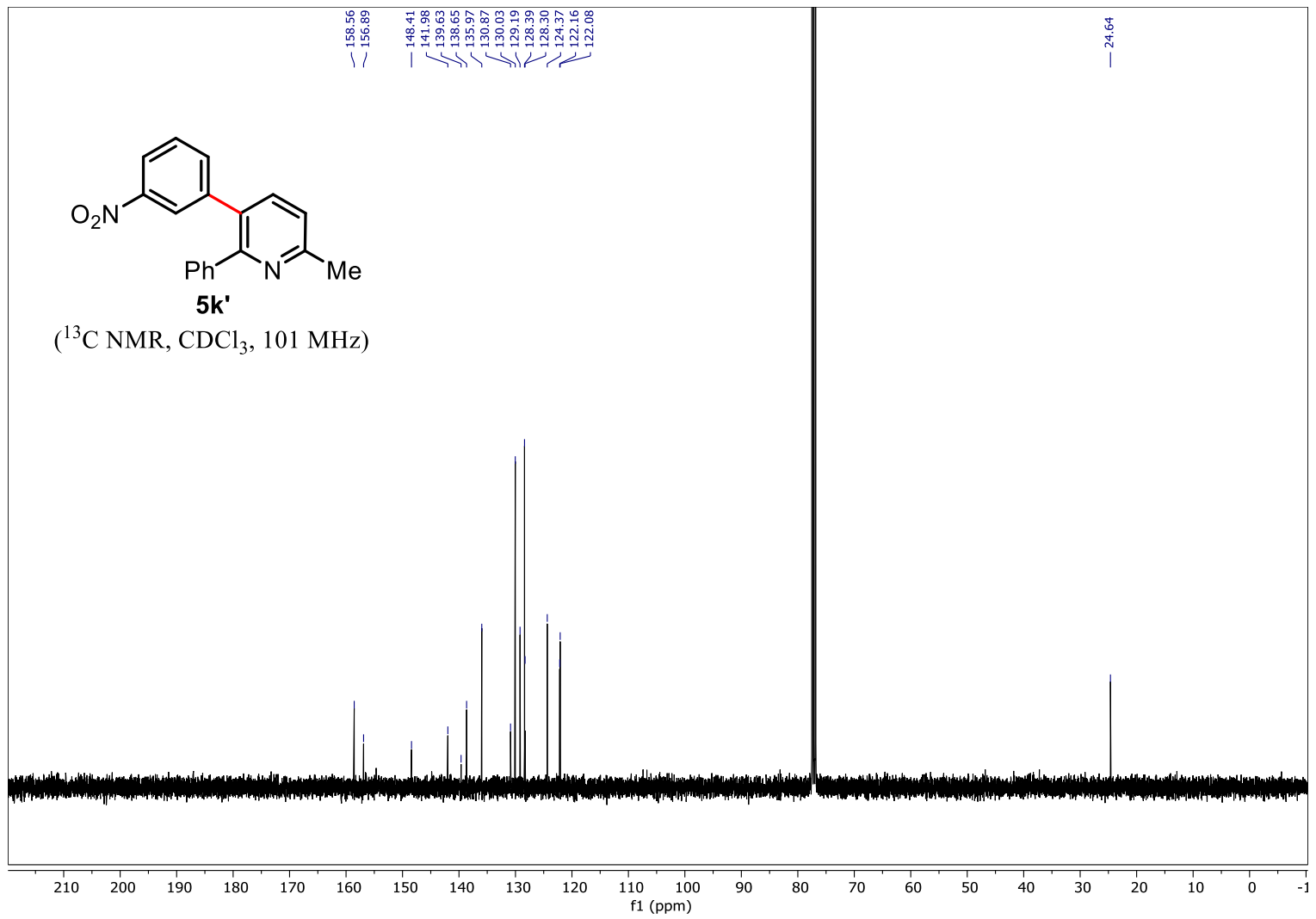

1D NOE NMR spectrum of $\mathbf{5} \mathbf{k}^{\prime}, 500 \mathrm{MHz}, \mathrm{C}_{6} \mathrm{D}_{6}$
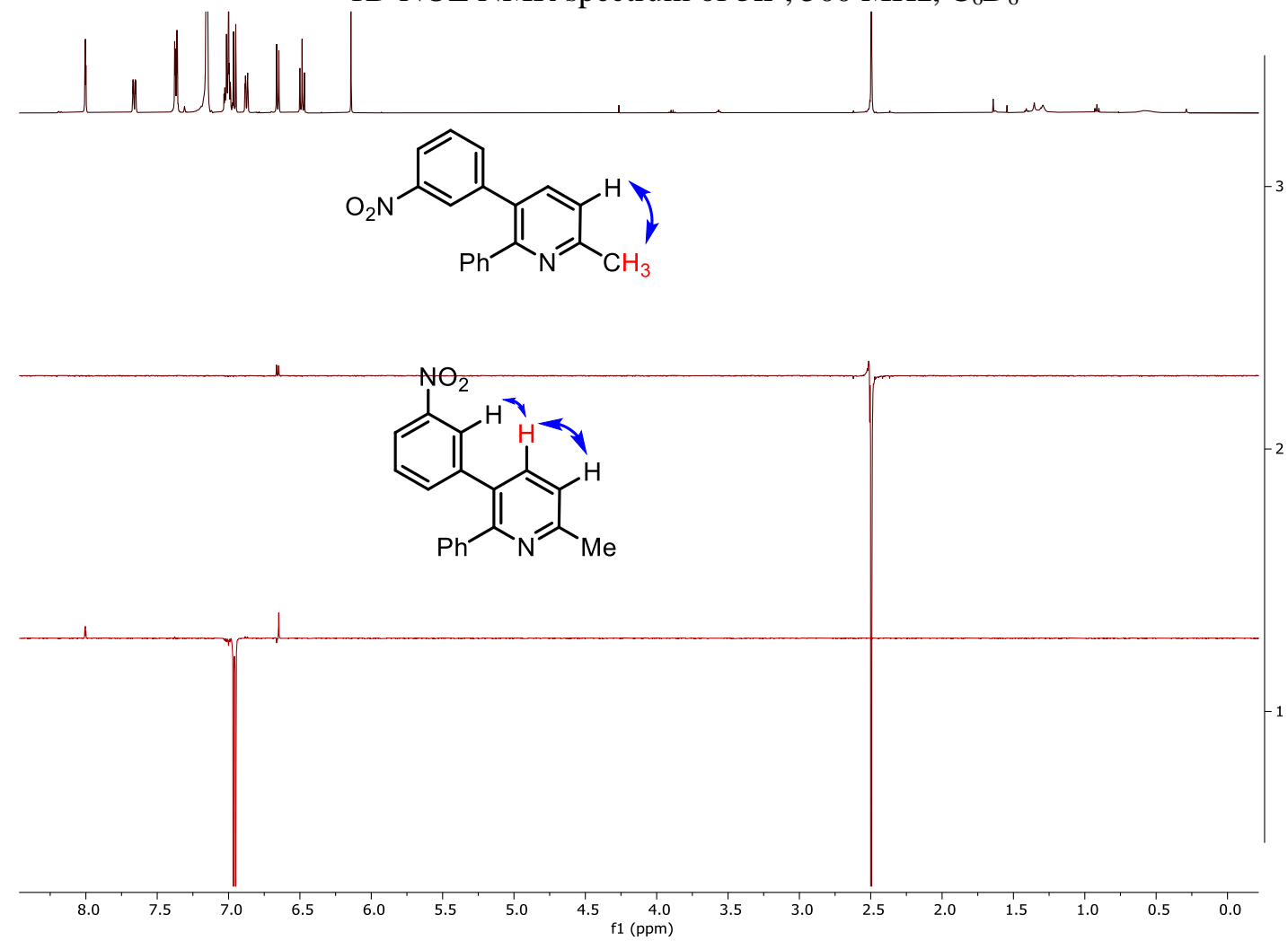

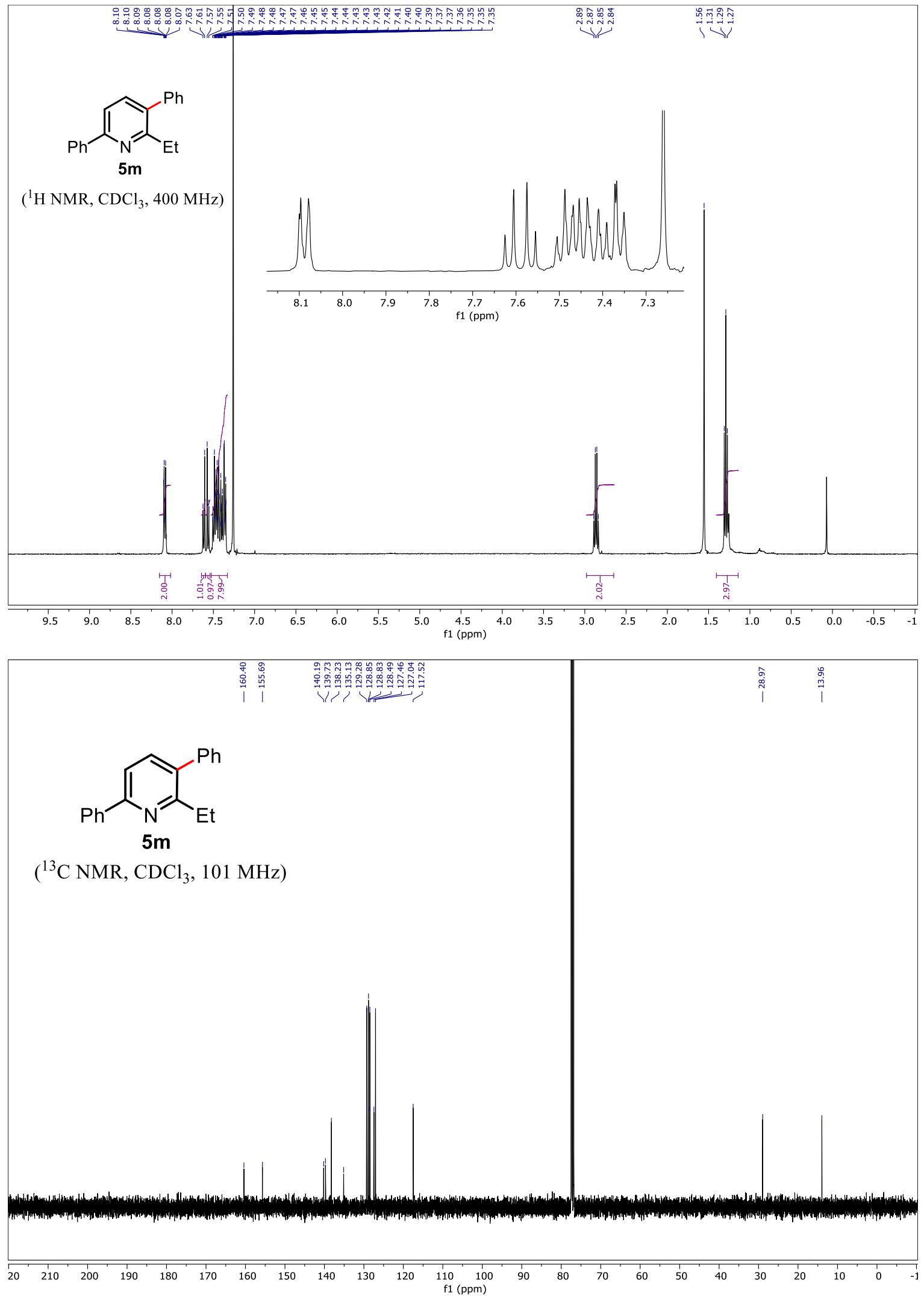
1D NOE NMR spectrum of $\mathbf{5 m}, 500 \mathrm{MHz}, \mathrm{CDCl}_{3}$
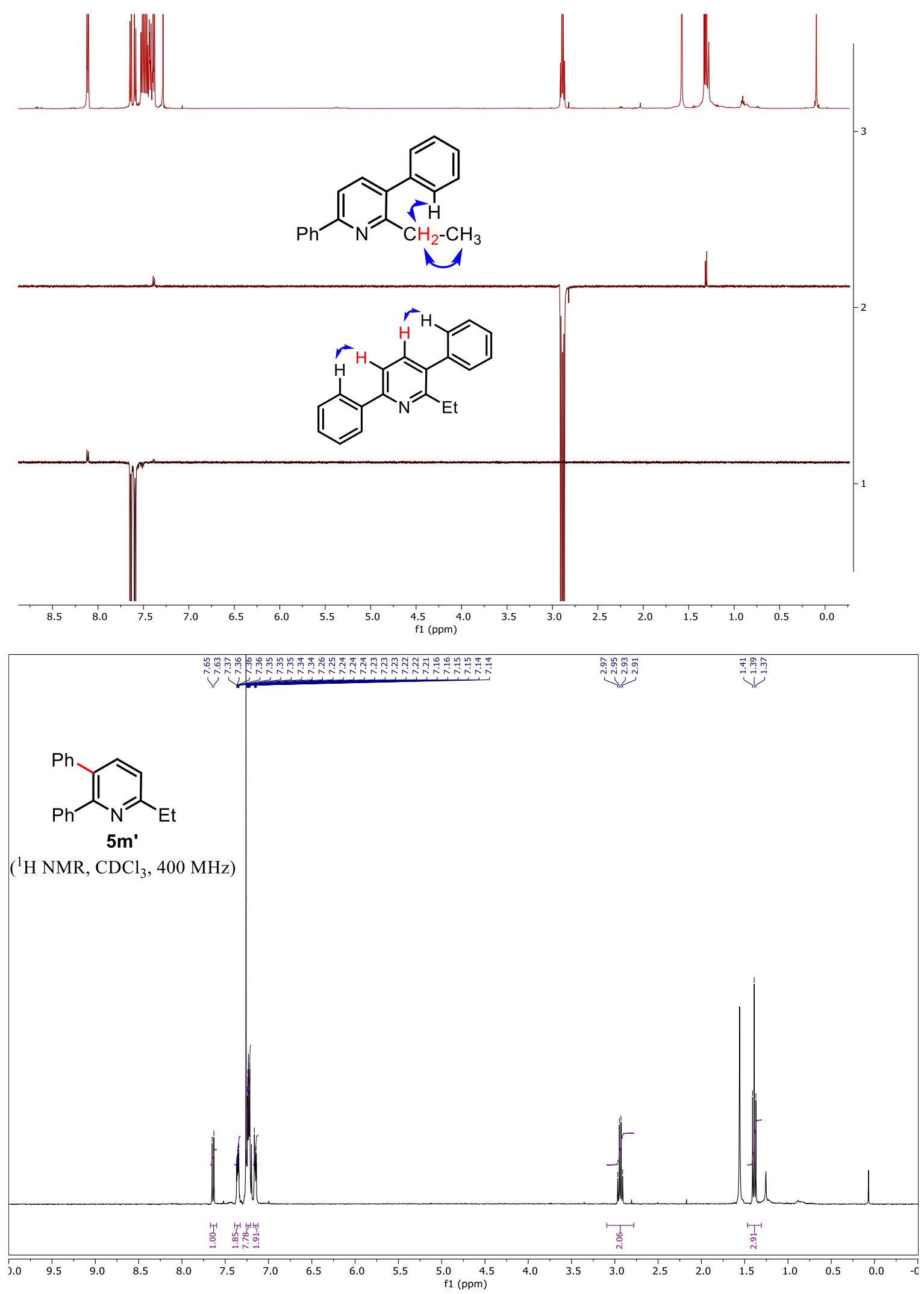


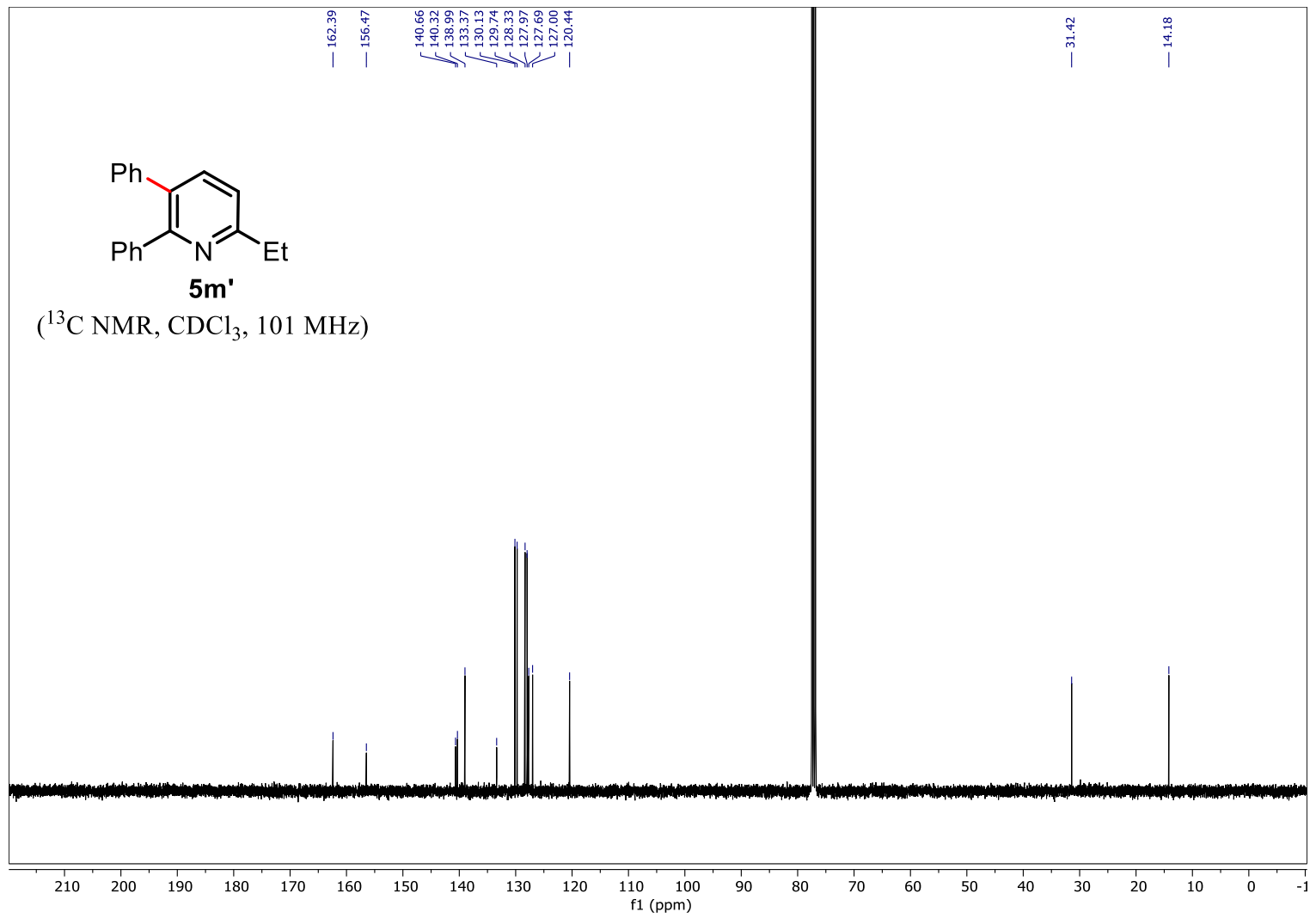

1D NOE NMR spectrum of 5m', $500 \mathrm{MHz}, \mathrm{C}_{6} \mathrm{D}_{6}$

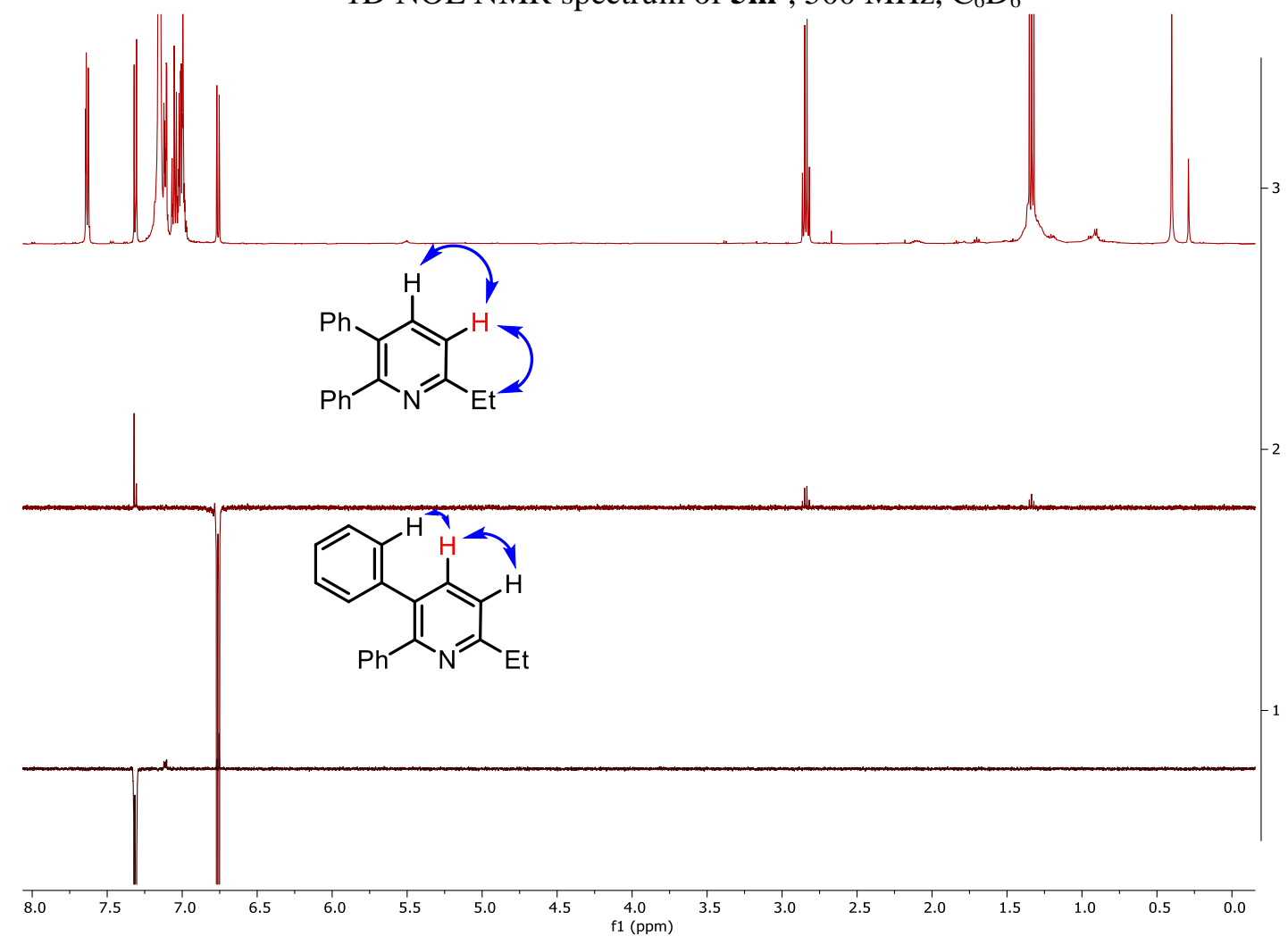



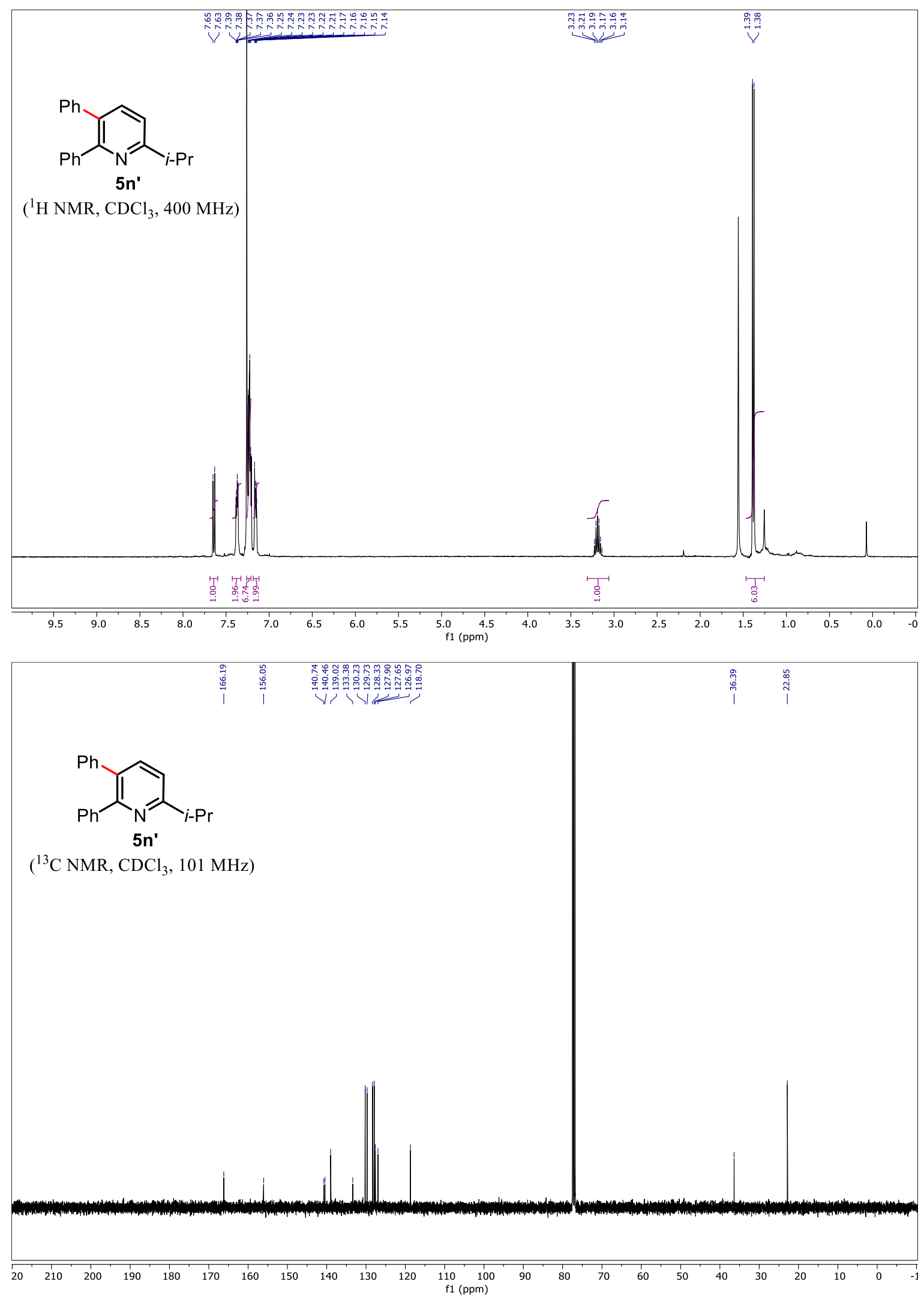

S1-89 
1D NOE NMR spectrum of 5n', $500 \mathrm{MHz}, \mathrm{C}_{6} \mathrm{D}_{6}$
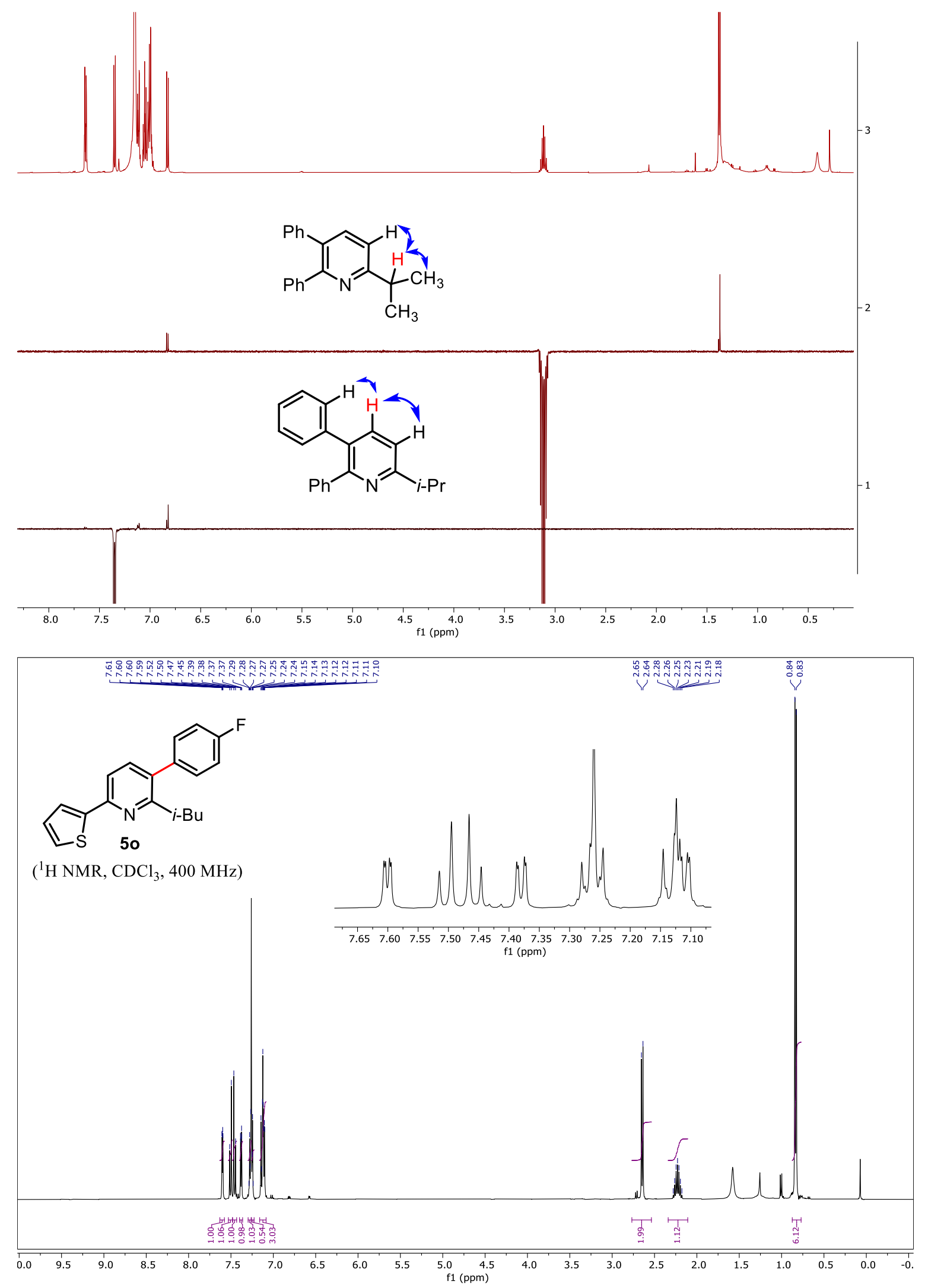

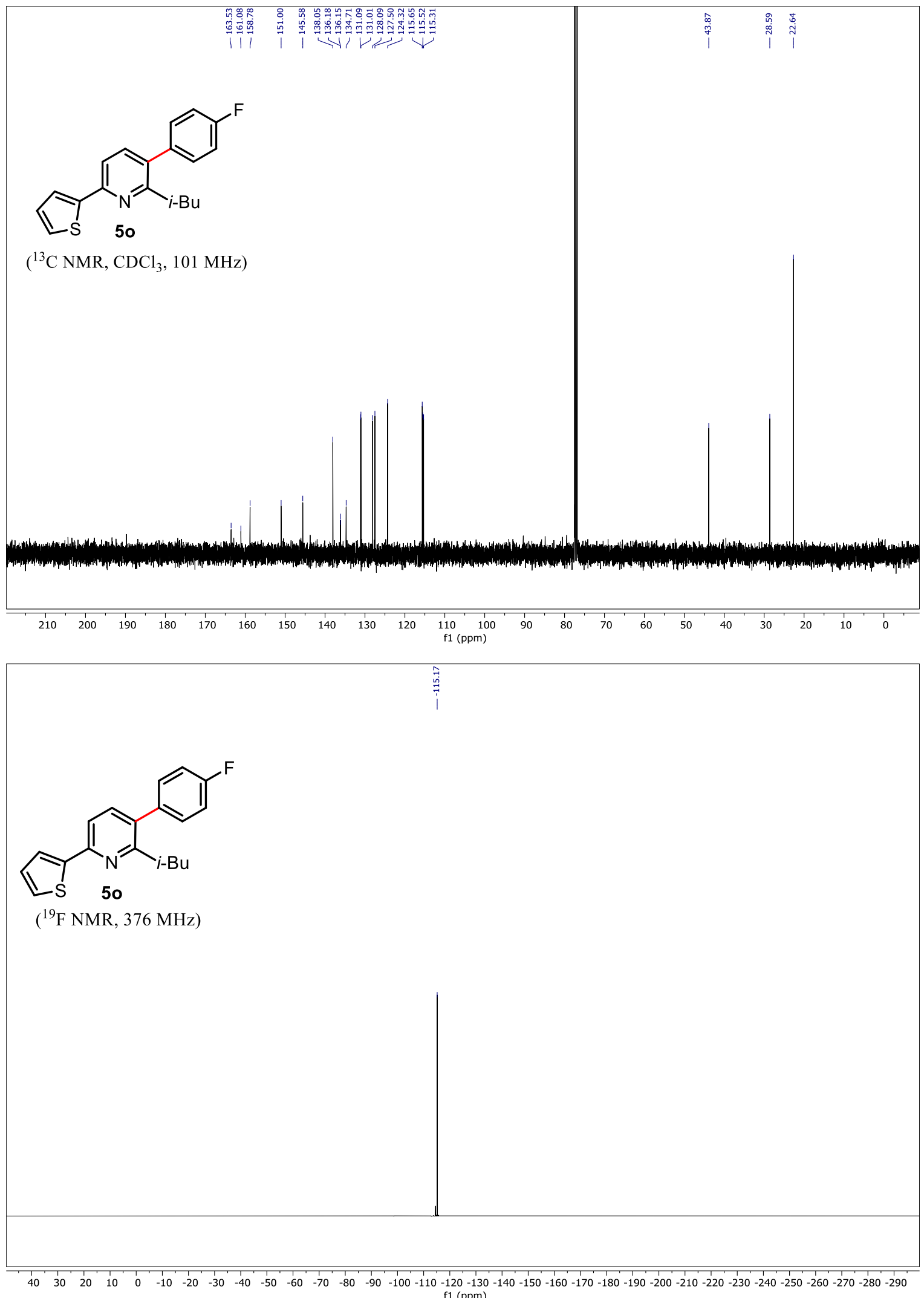
1D NOE NMR spectrum of $\mathbf{5 o}, 500 \mathrm{MHz}, \mathrm{CDCl}_{3}$
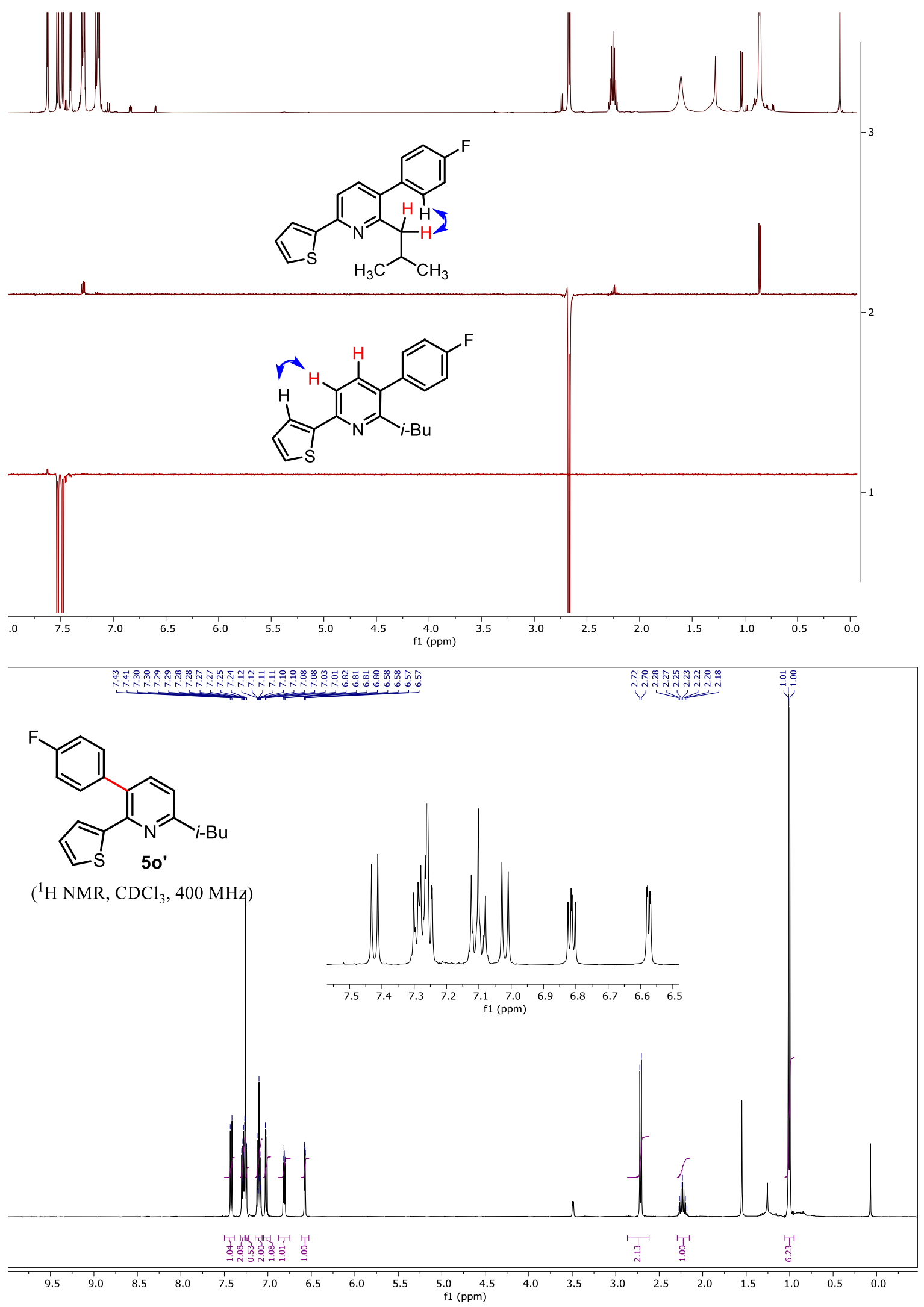

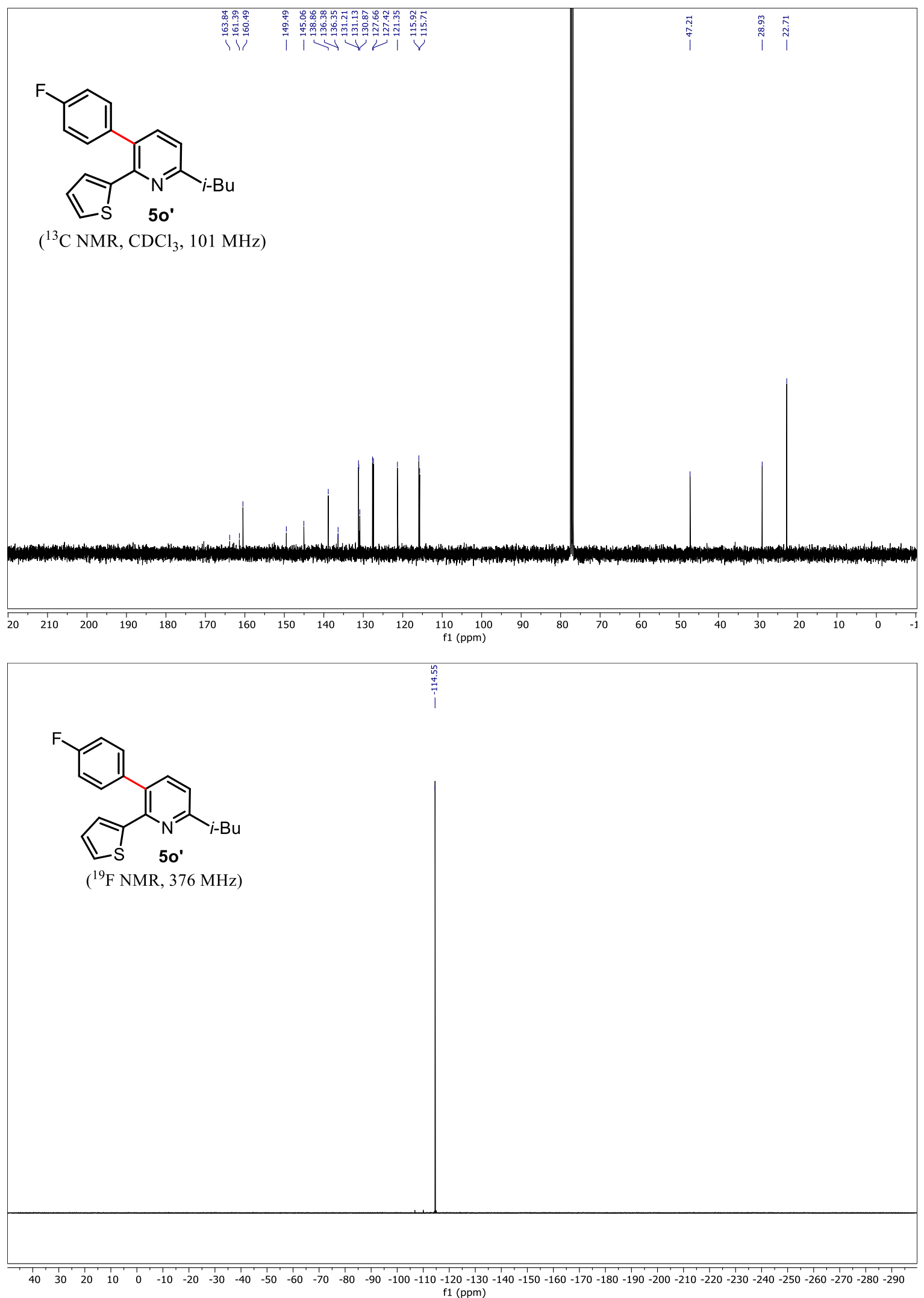
f1 (ppm) 
1D NOE NMR spectrum of 5o', $500 \mathrm{MHz}, \mathrm{CDCl}_{3}$
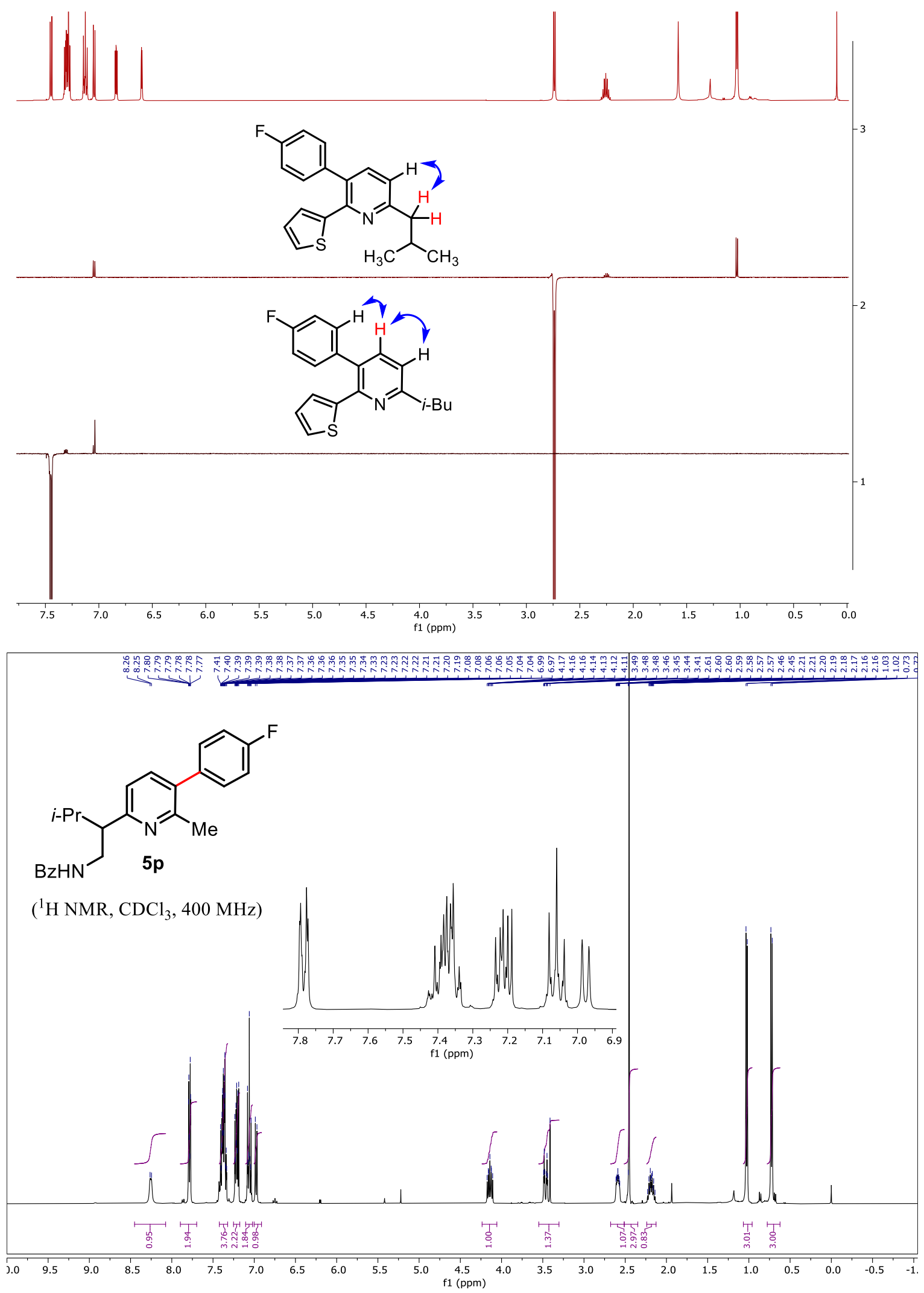

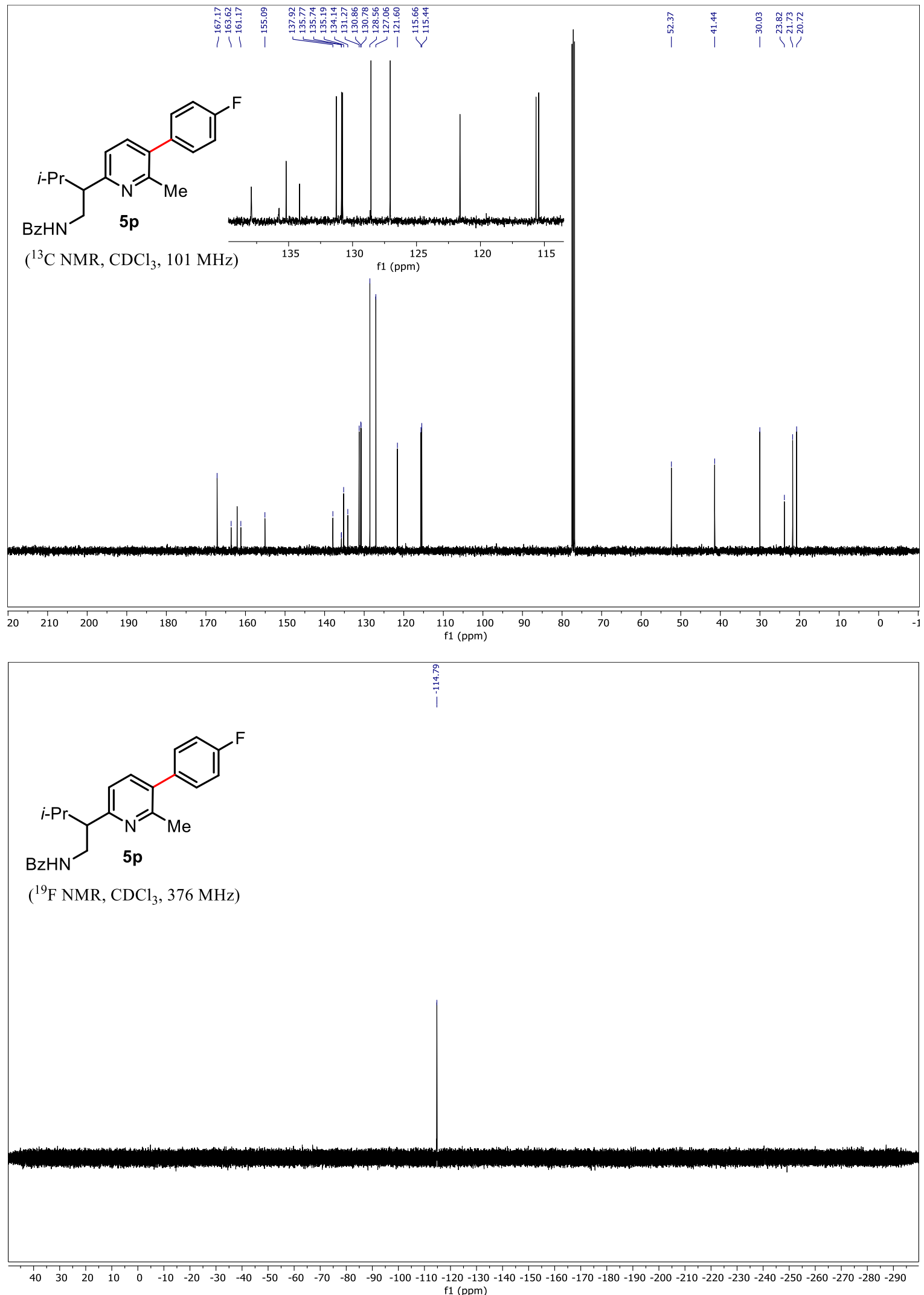
1D NOE NMR spectrum of 5p, $500 \mathrm{MHz}, \mathrm{C}_{6} \mathrm{D}_{6}$
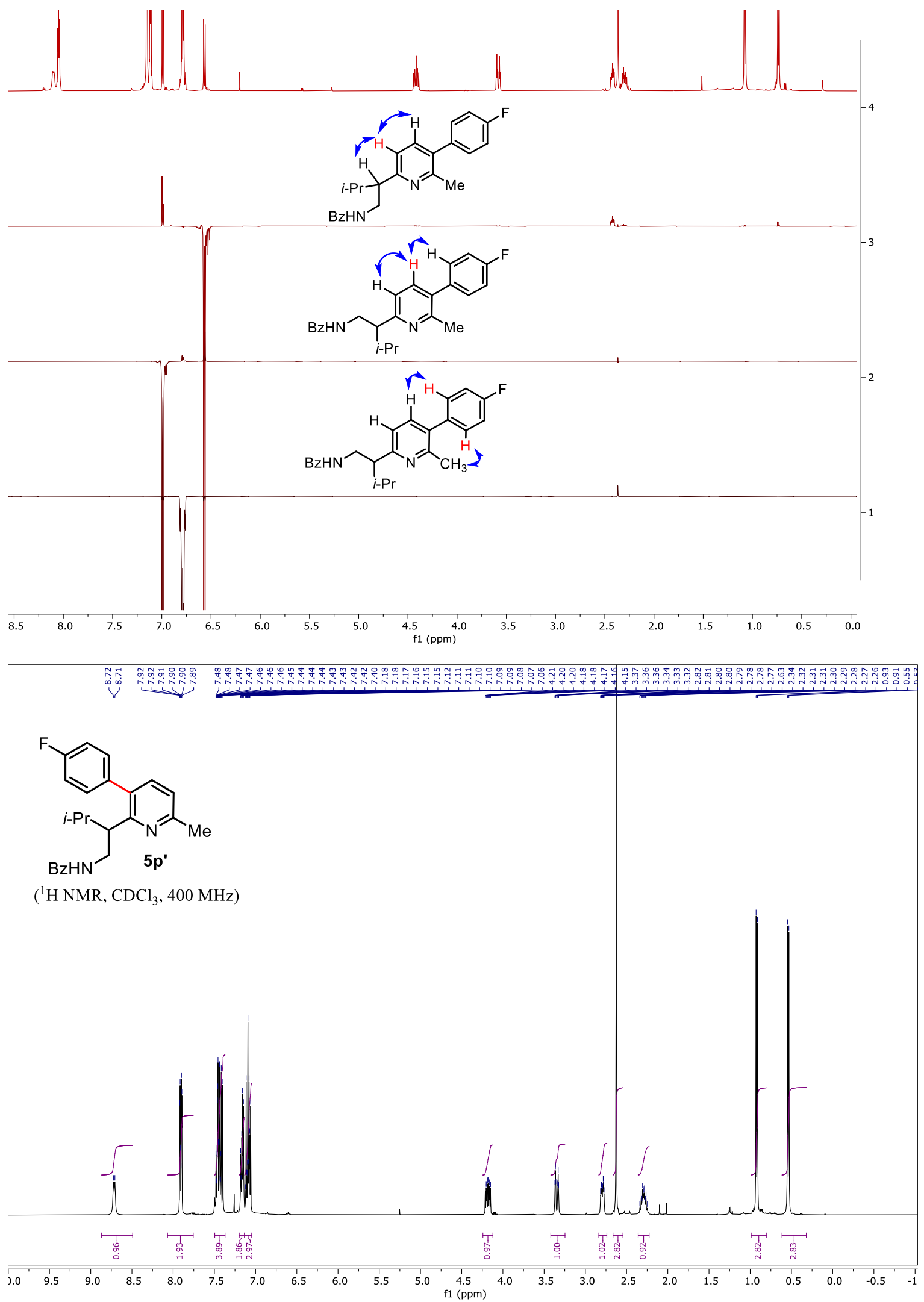

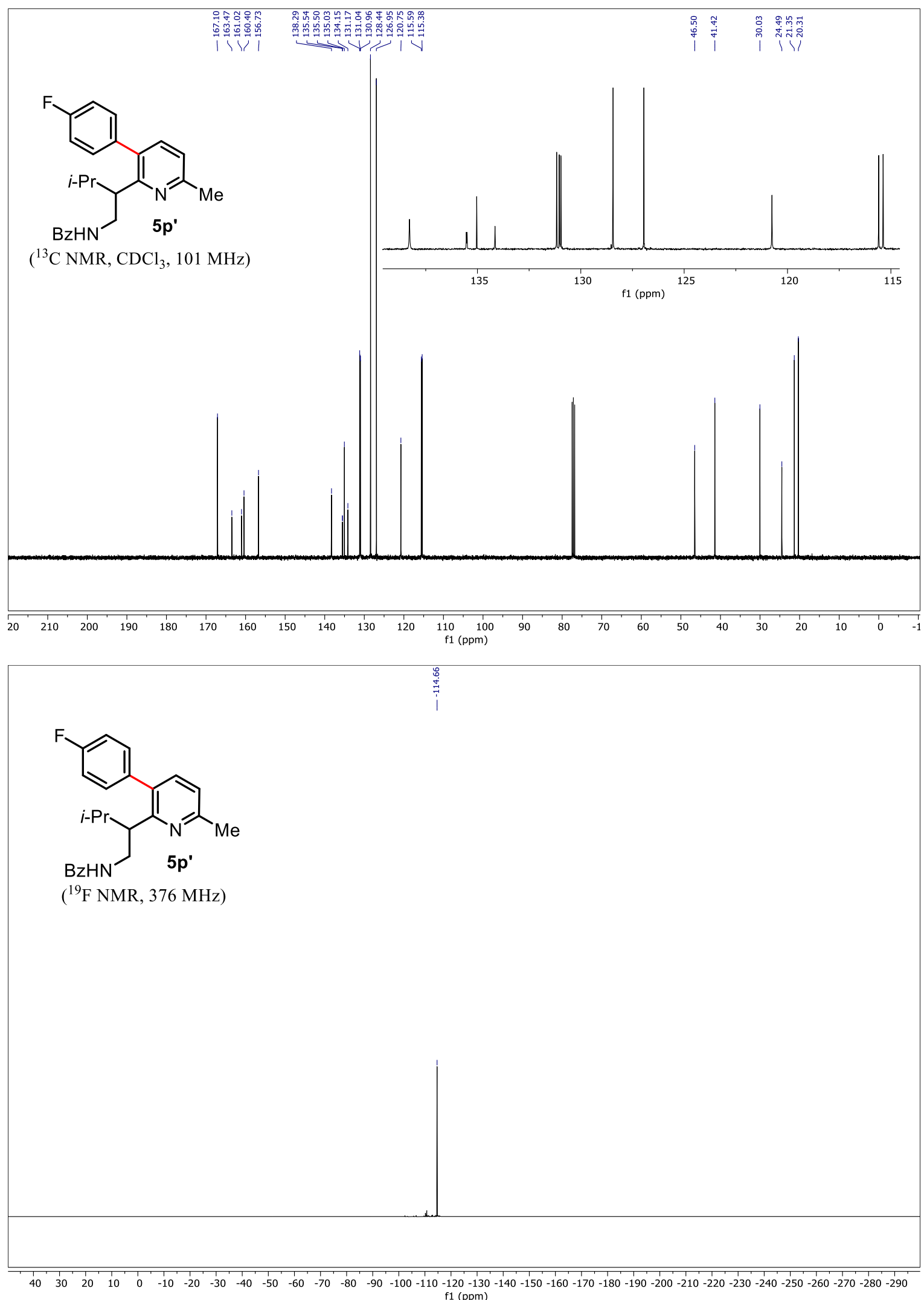
f1 (ppm) 
1D NOE NMR spectrum of 5p', $500 \mathrm{MHz}, \mathrm{C}_{6} \mathrm{D}_{6}$
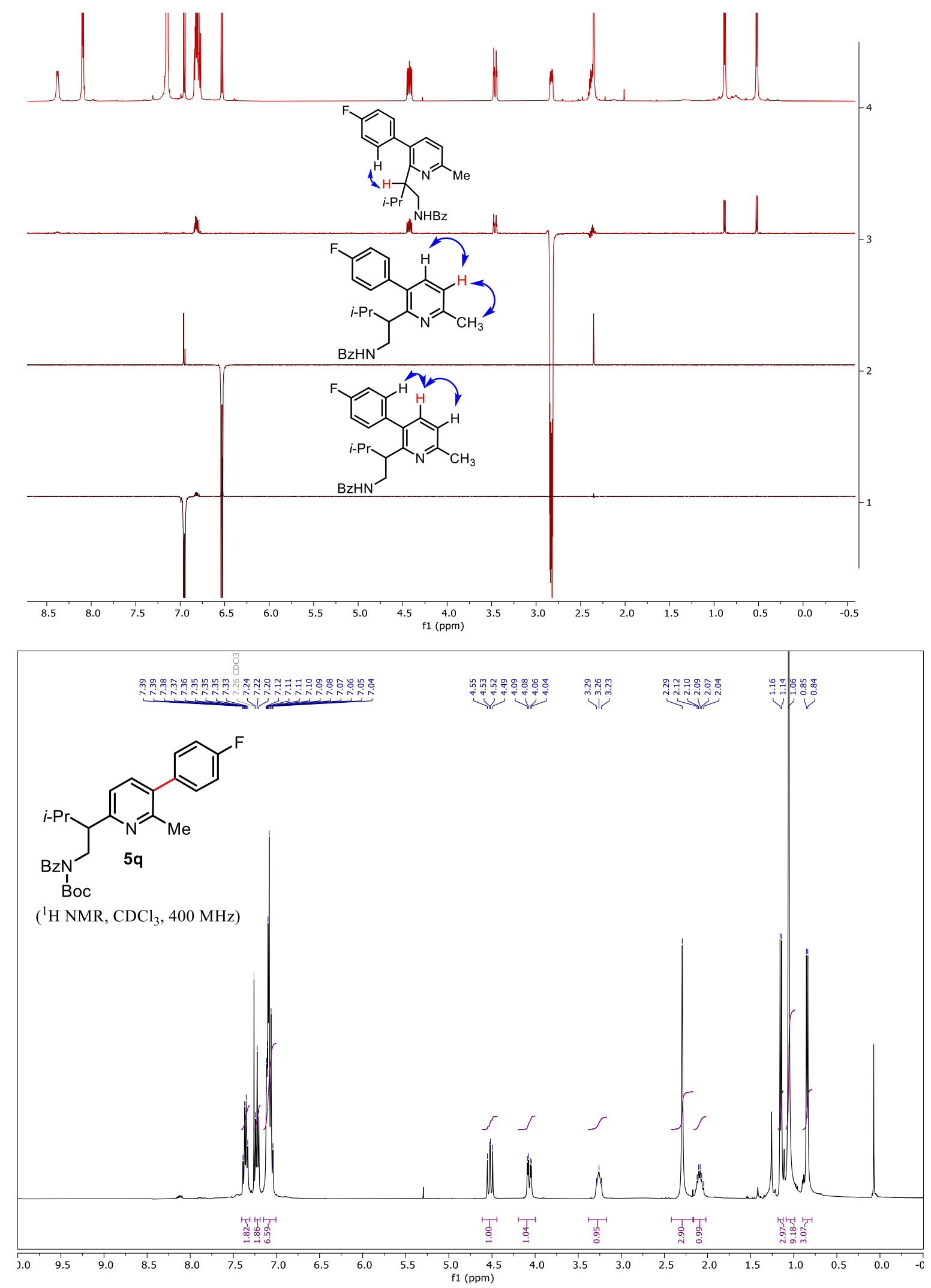

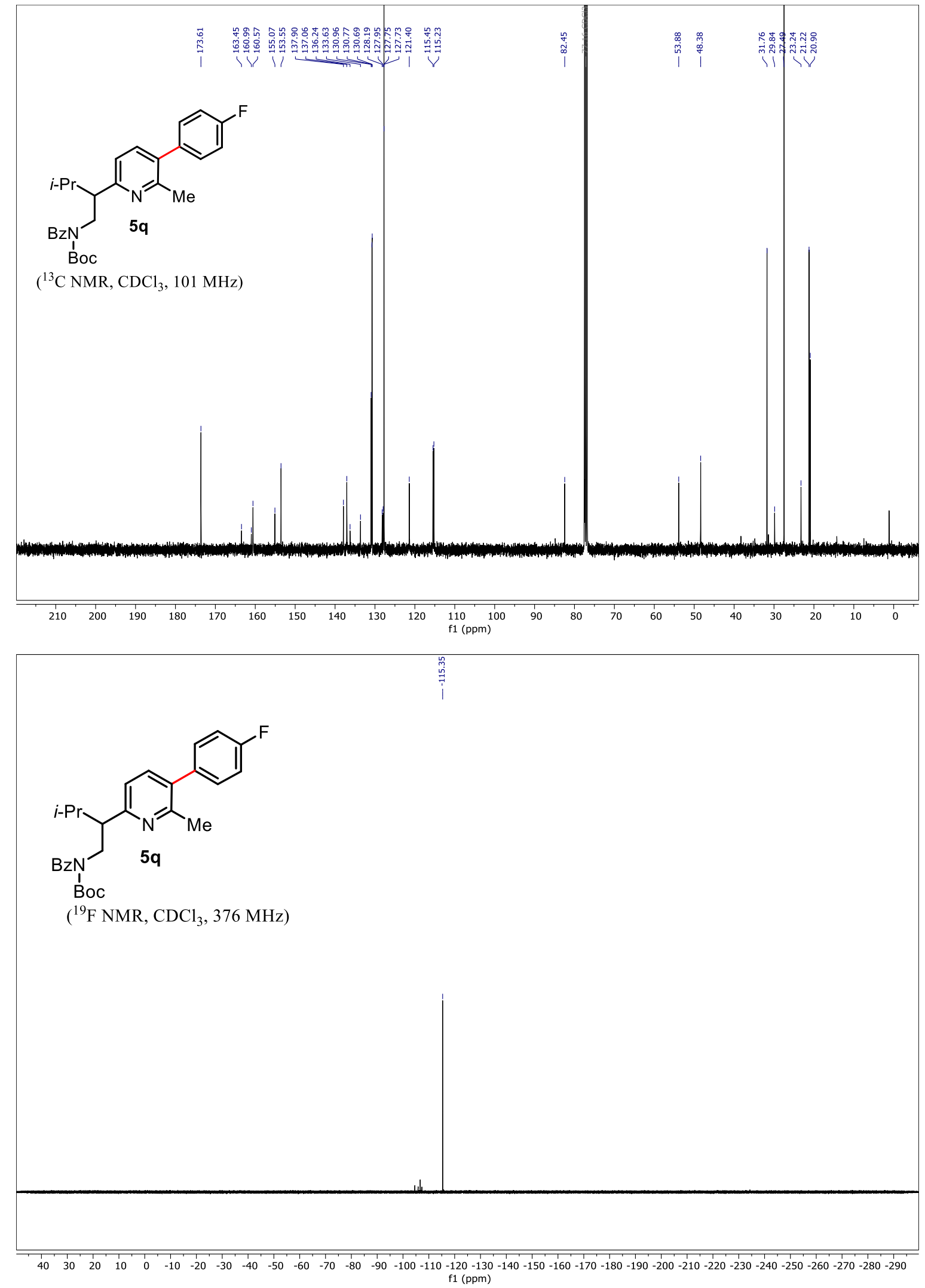
1D NOE NMR spectrum of $\mathbf{5 q}, 500 \mathrm{MHz}, \mathrm{C}_{6} \mathrm{D}_{6}$
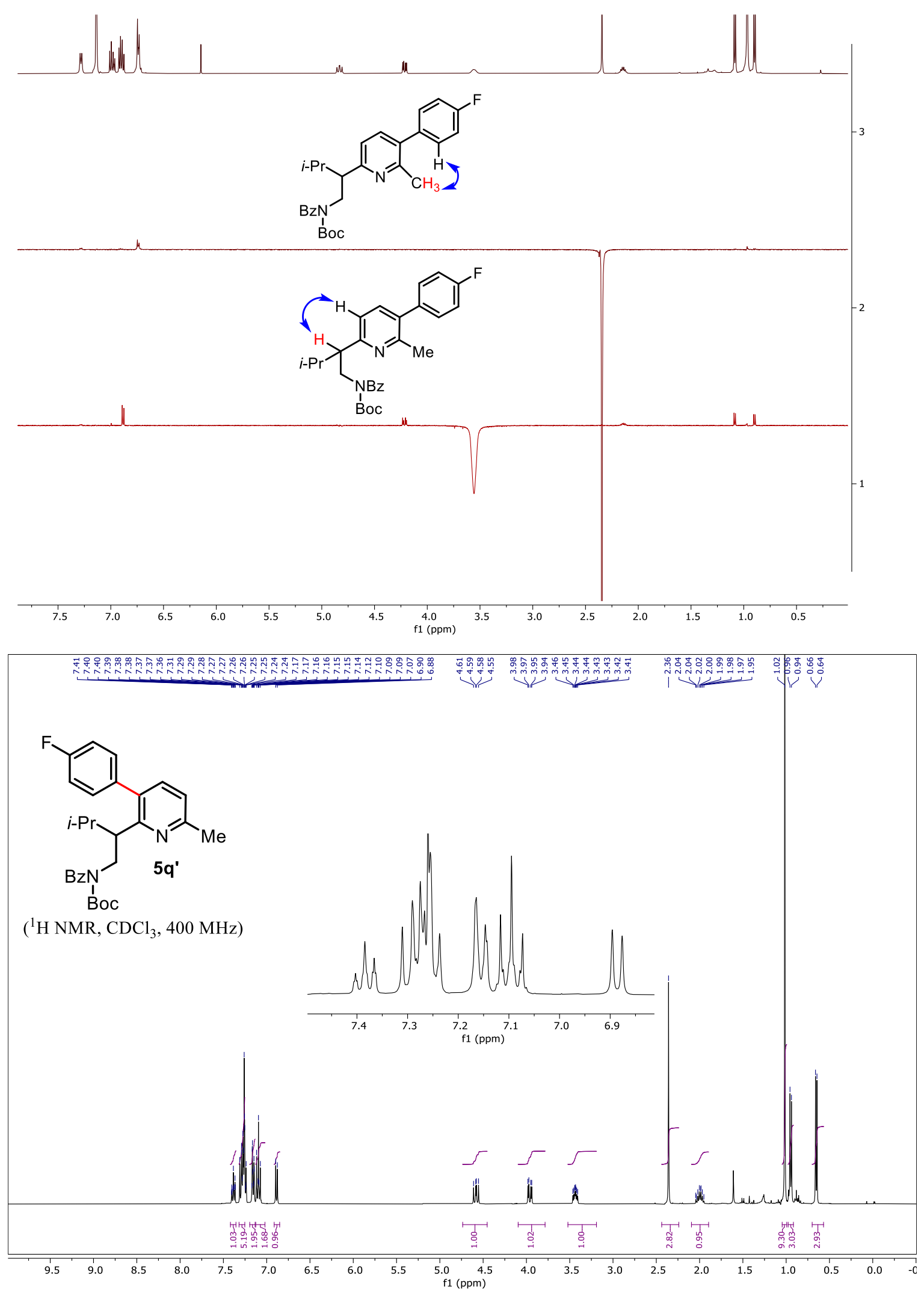

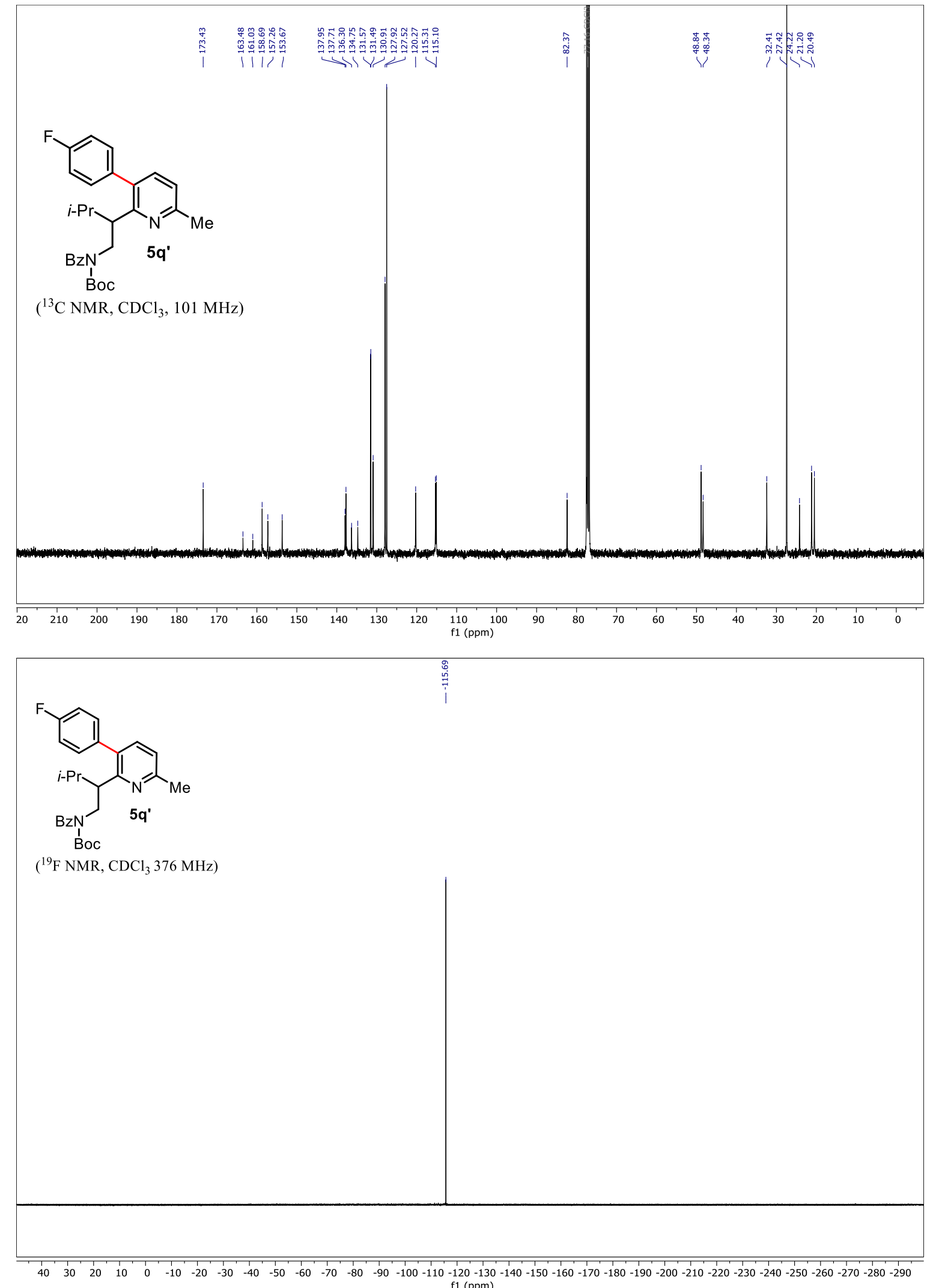


\section{D NOE NMR spectrum of 5q', $500 \mathrm{MHz}, \mathrm{CDCl}_{3}$}
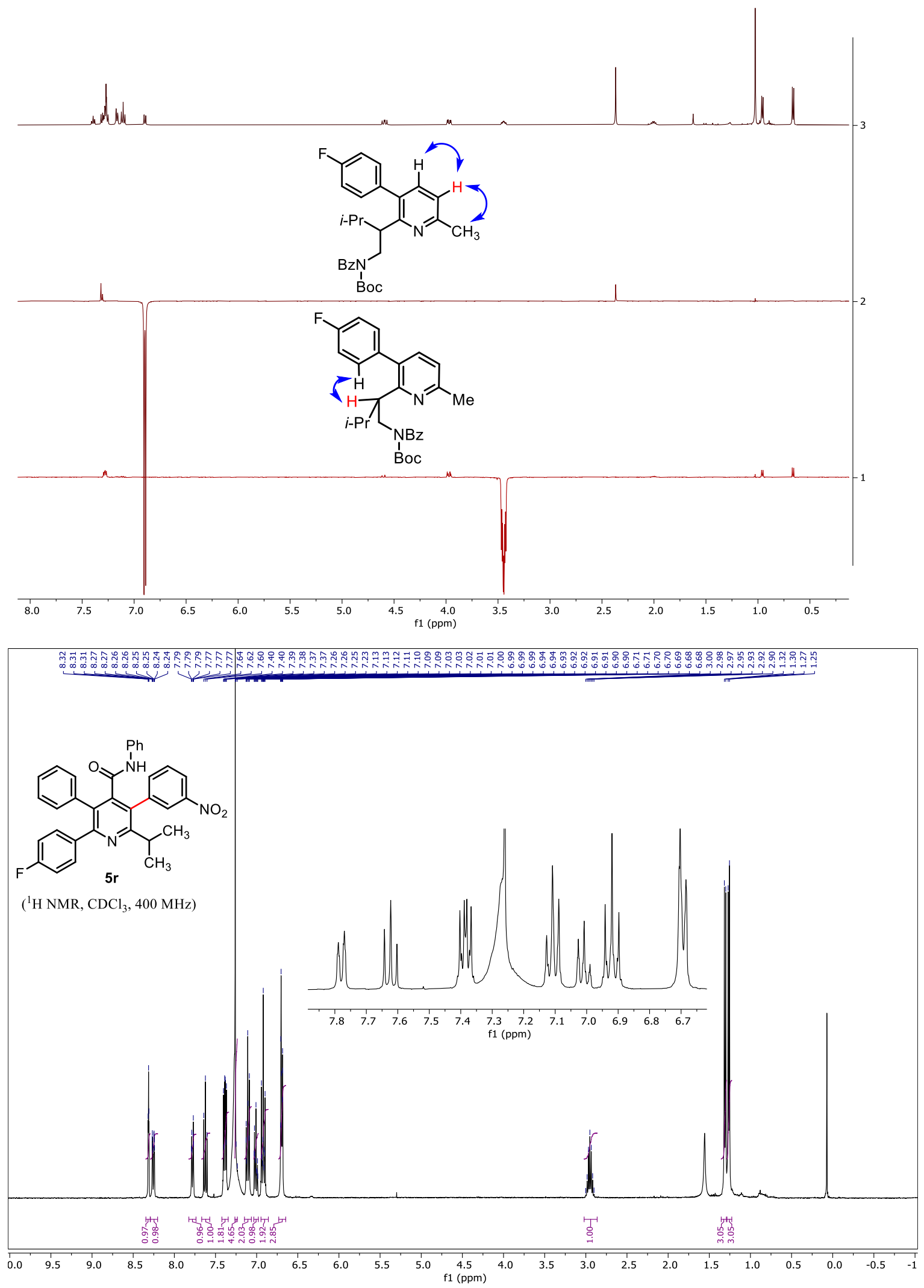

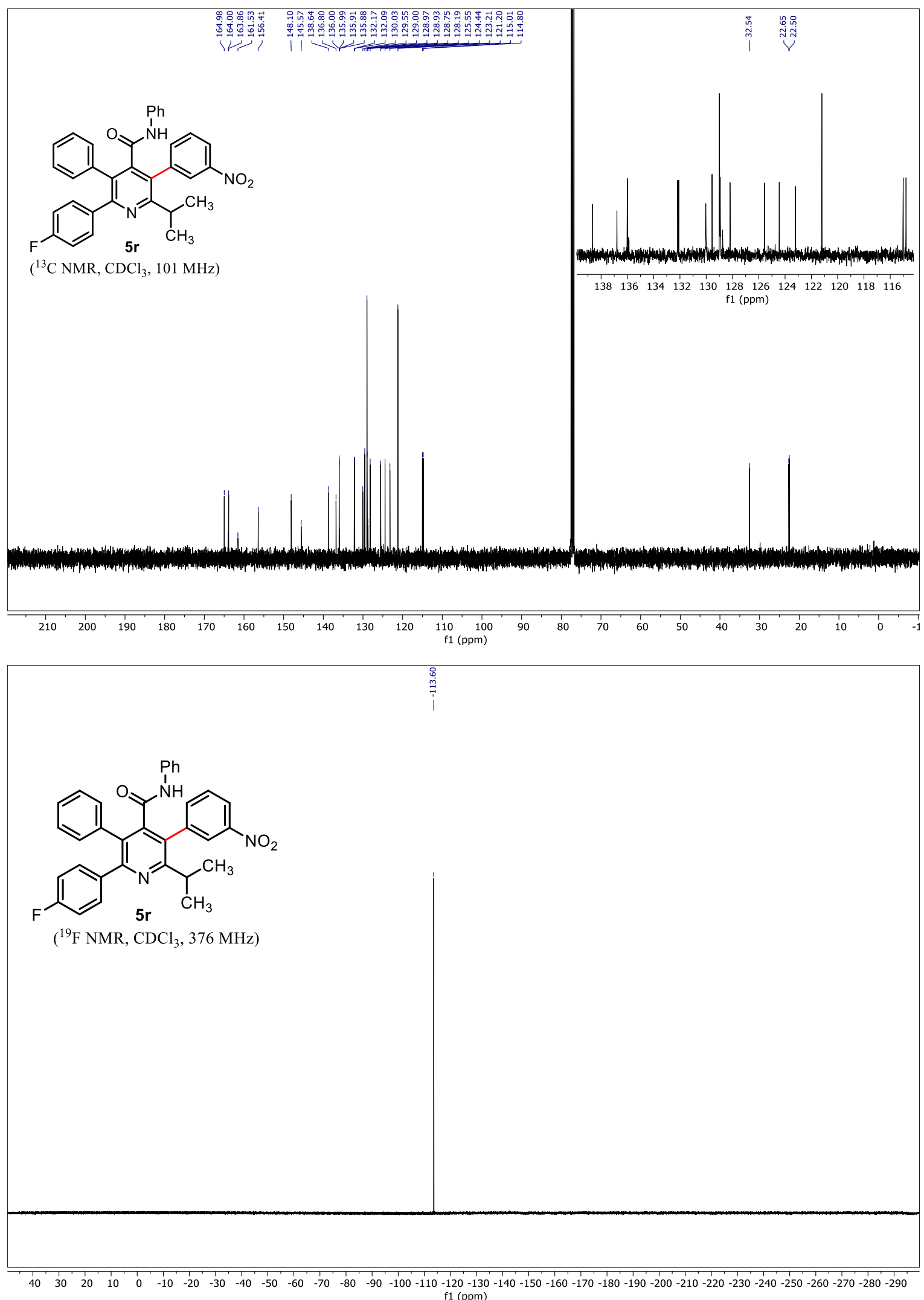
f1 (ppm) 
1D NOE NMR spectrum of $\mathbf{5 r}, 500 \mathrm{MHz}, \mathrm{CDCl}_{3}$
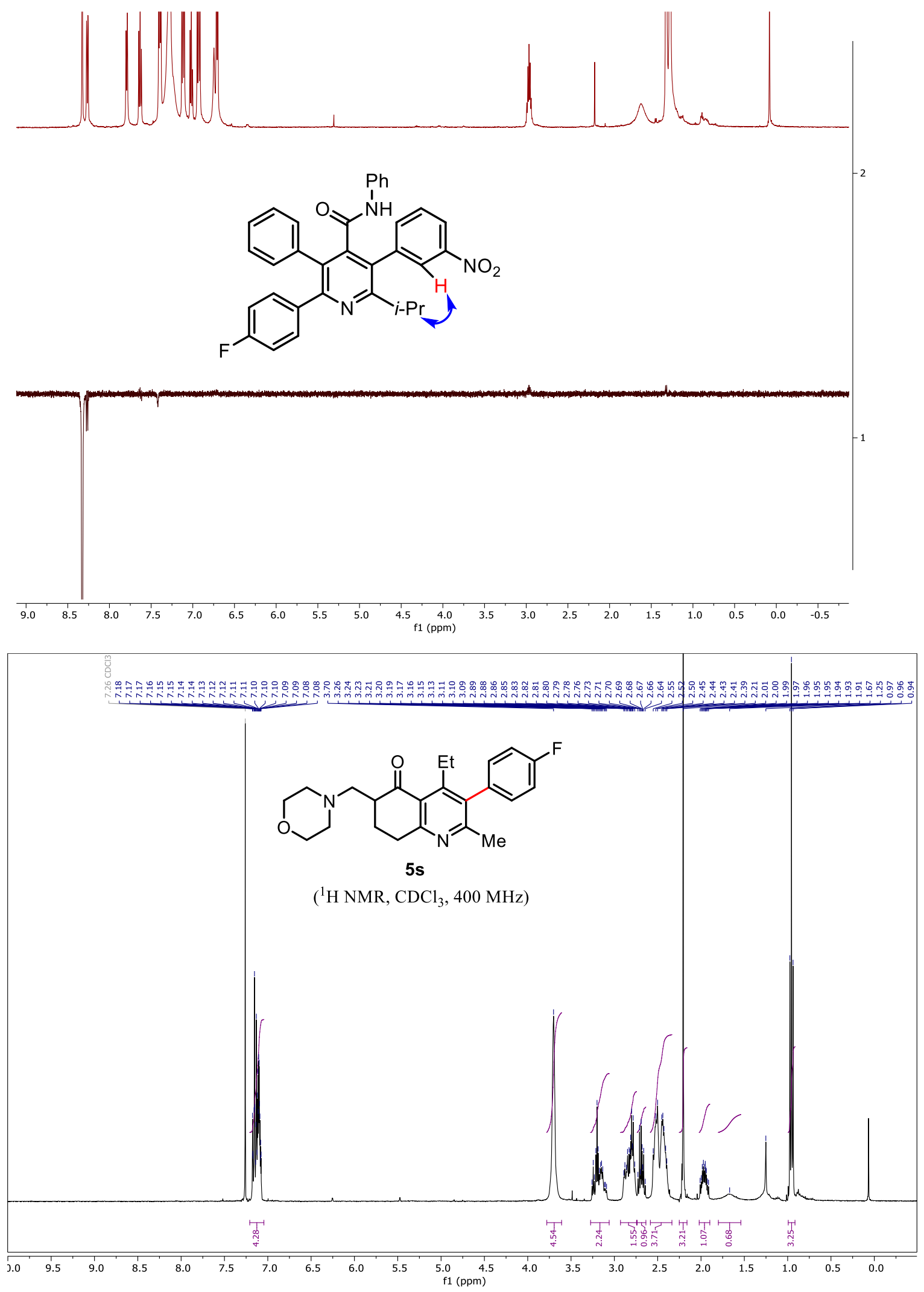

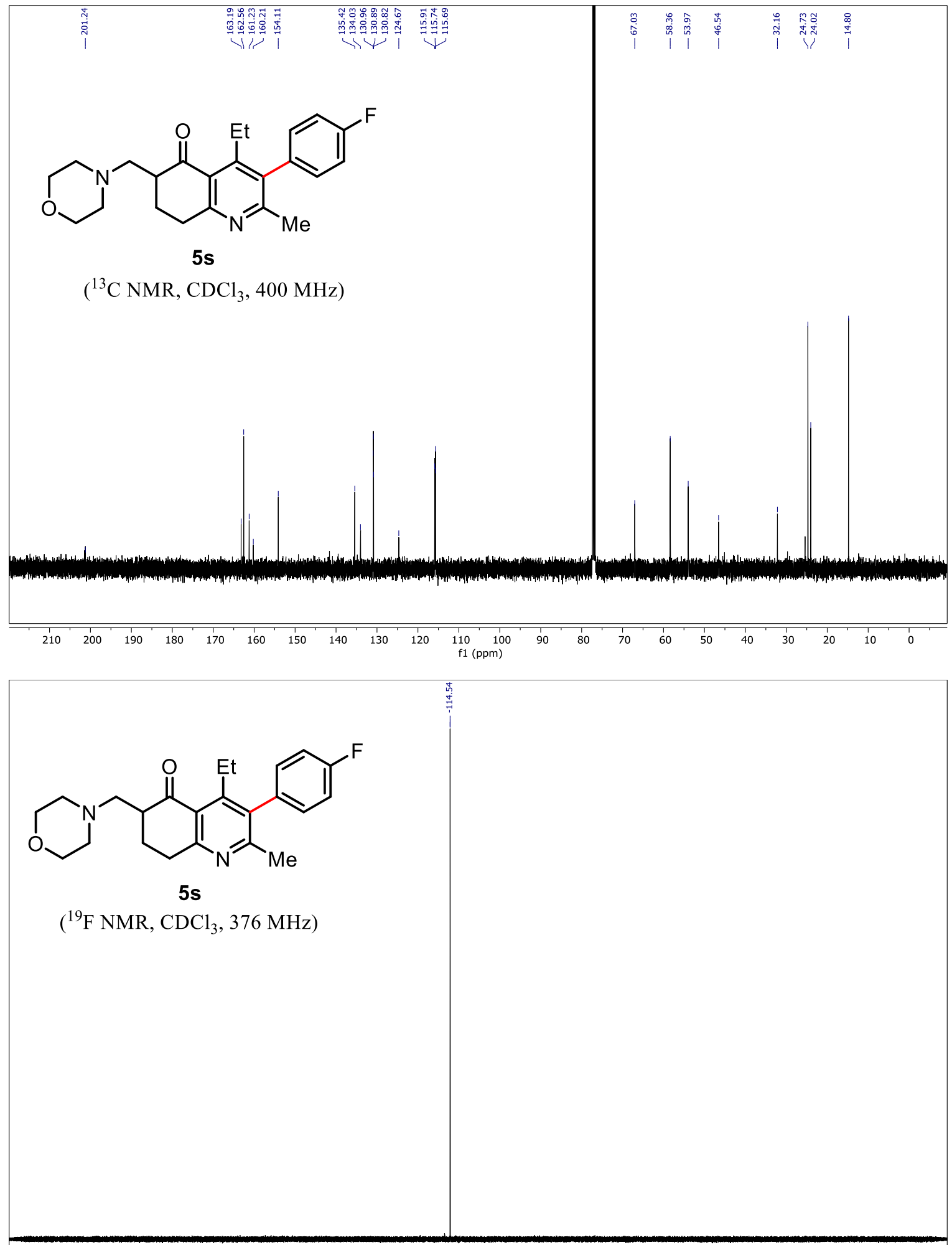

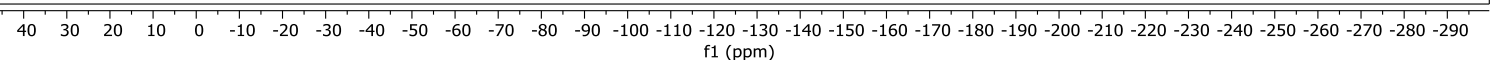



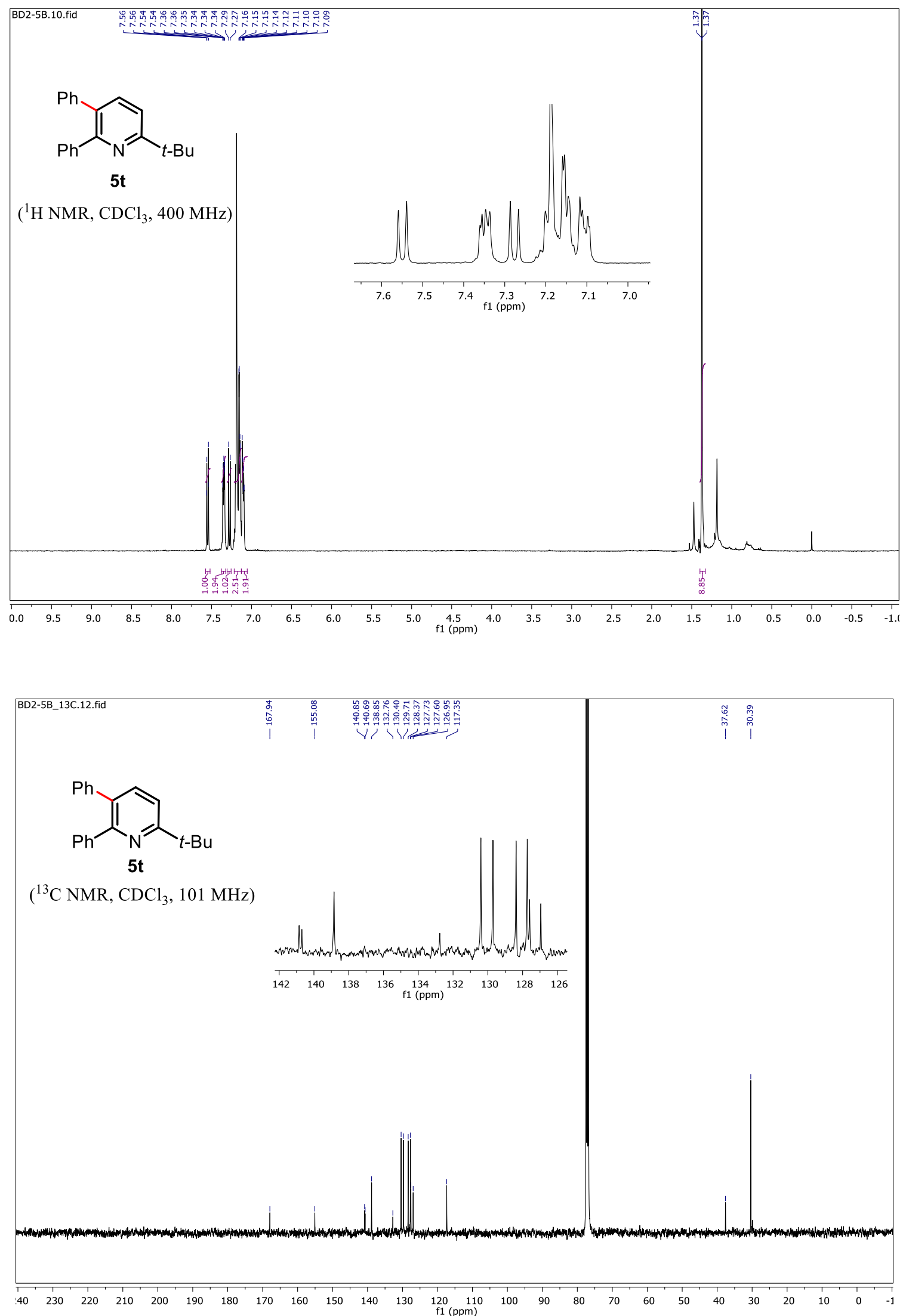
1D NOE NMR spectrum of $\mathbf{5 t}, 500 \mathrm{MHz}, \mathrm{CDCl}_{3}$
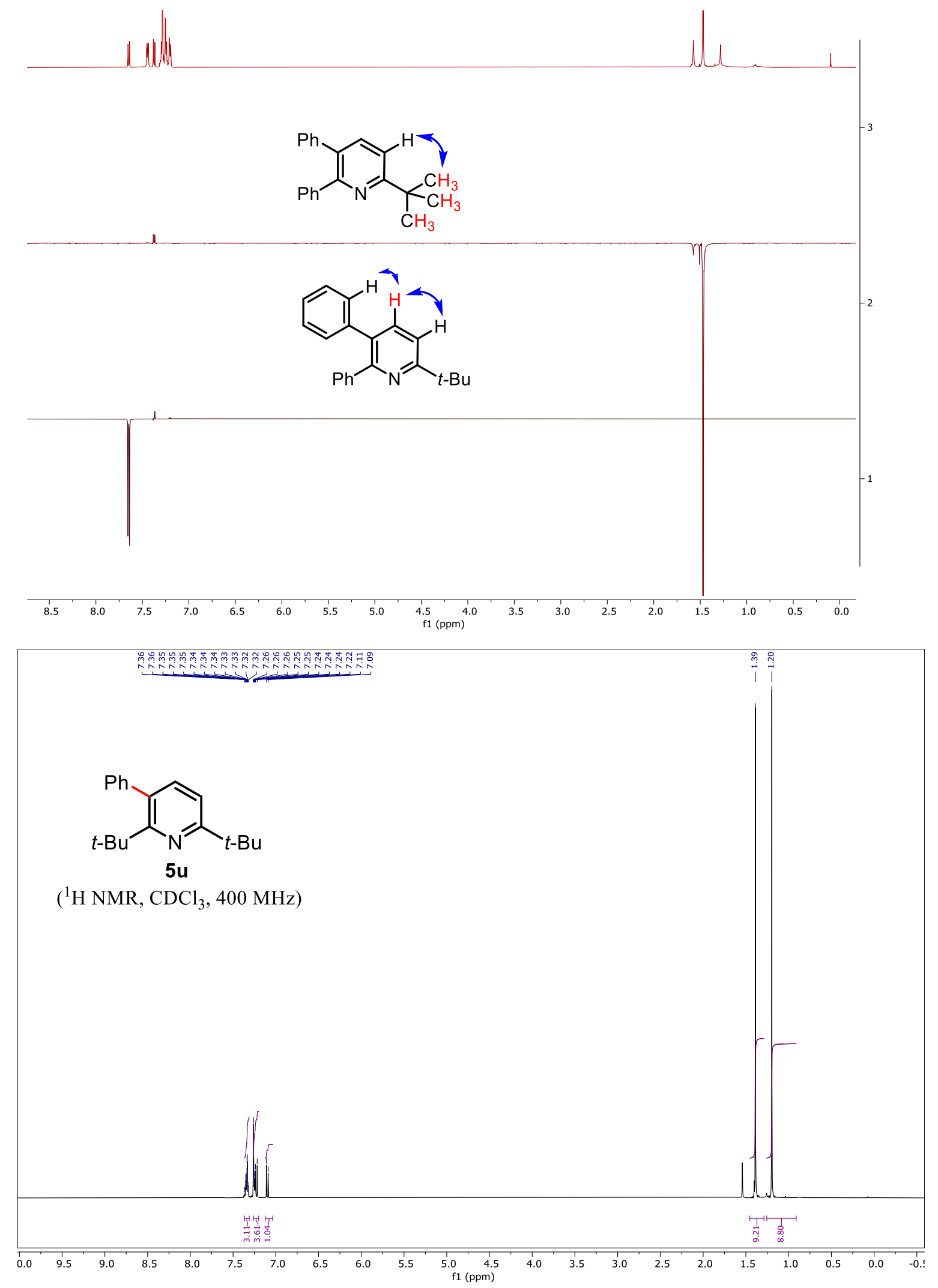


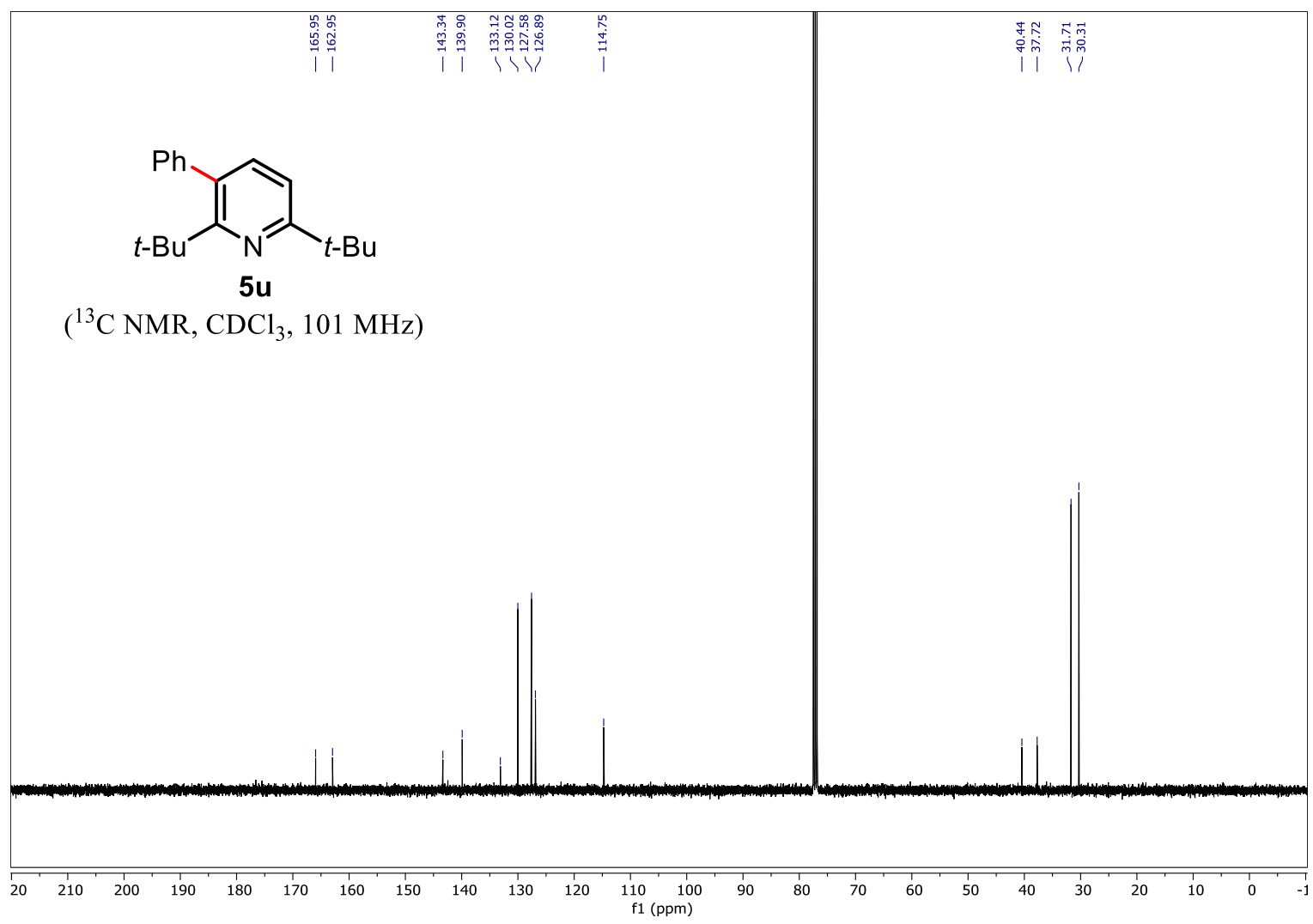

1D NOE NMR spectrum of $\mathbf{5 u}, 500 \mathrm{MHz}, \mathrm{CDCl}_{3}$

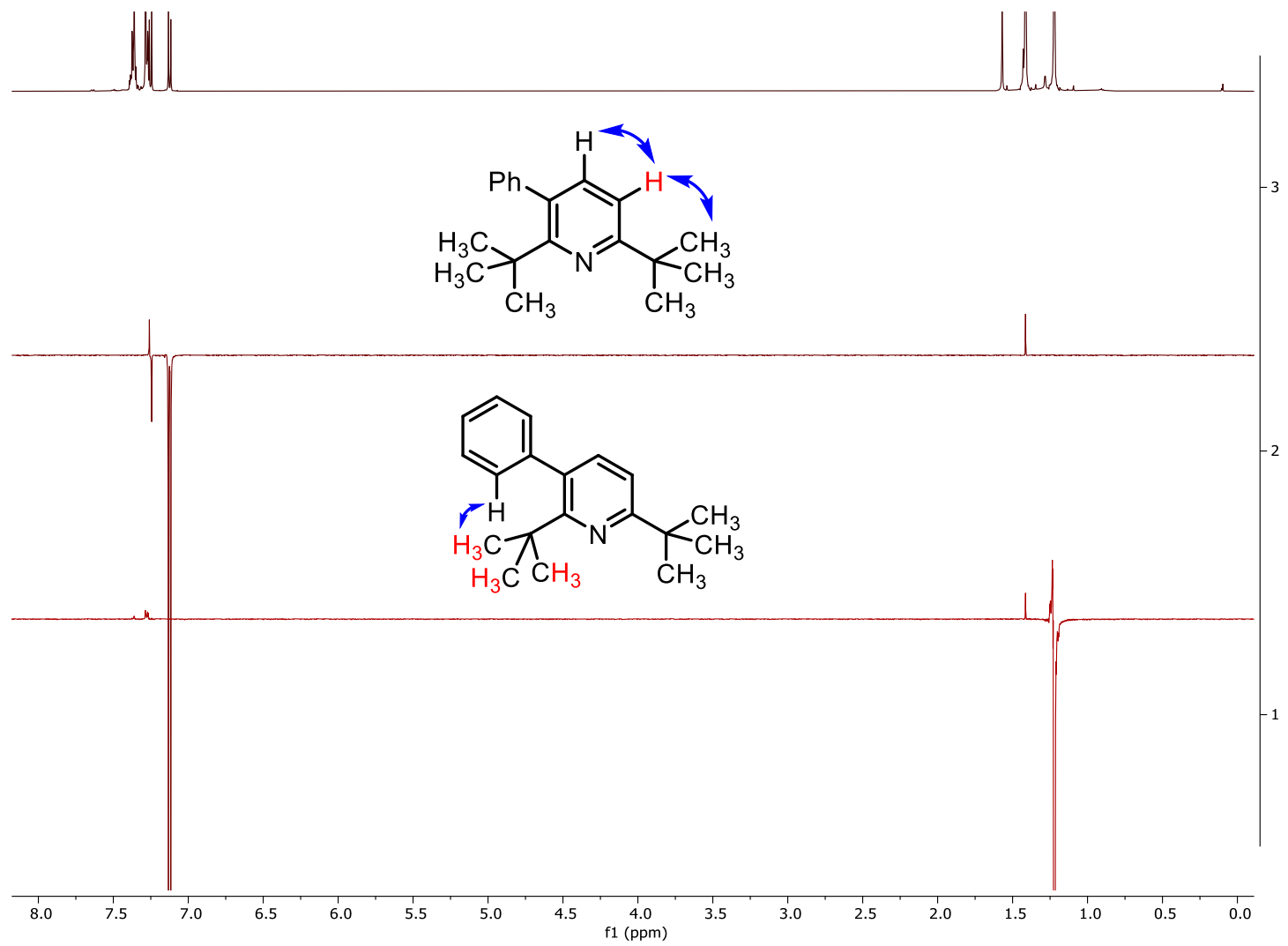



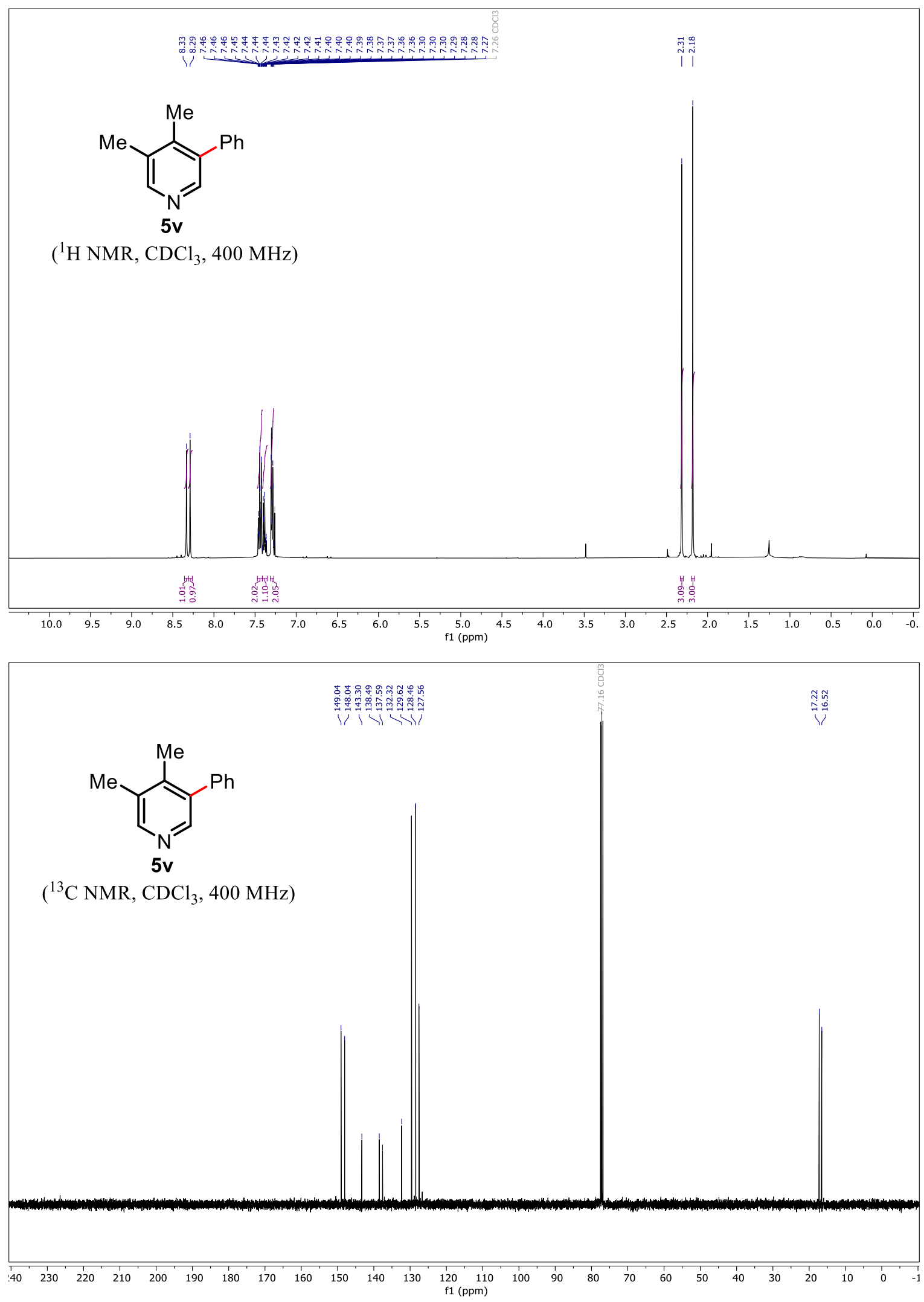

1D NOE NMR spectrum of $\mathbf{5 v}, 500 \mathrm{MHz}, \mathrm{CDCl}_{3}$ 

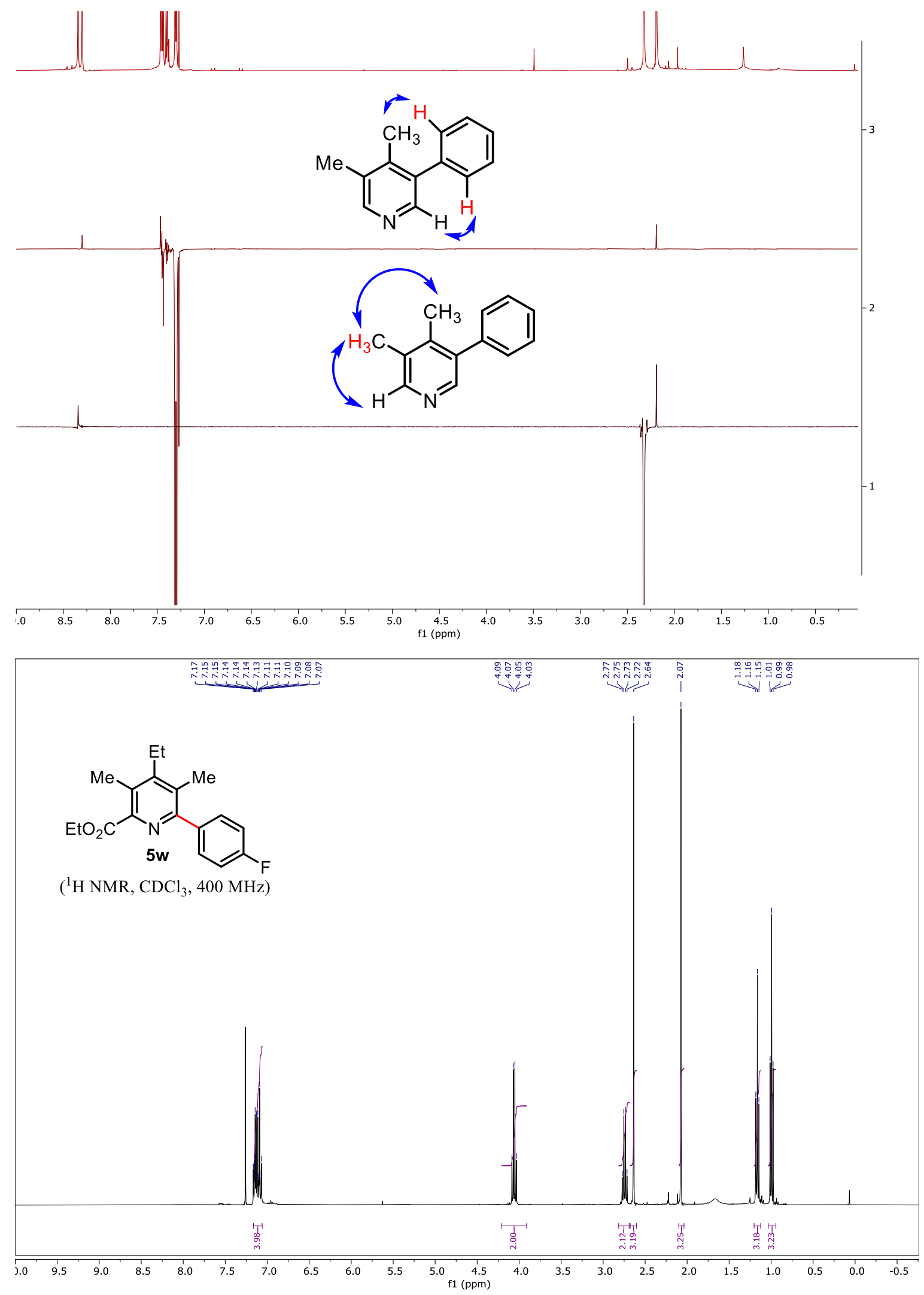

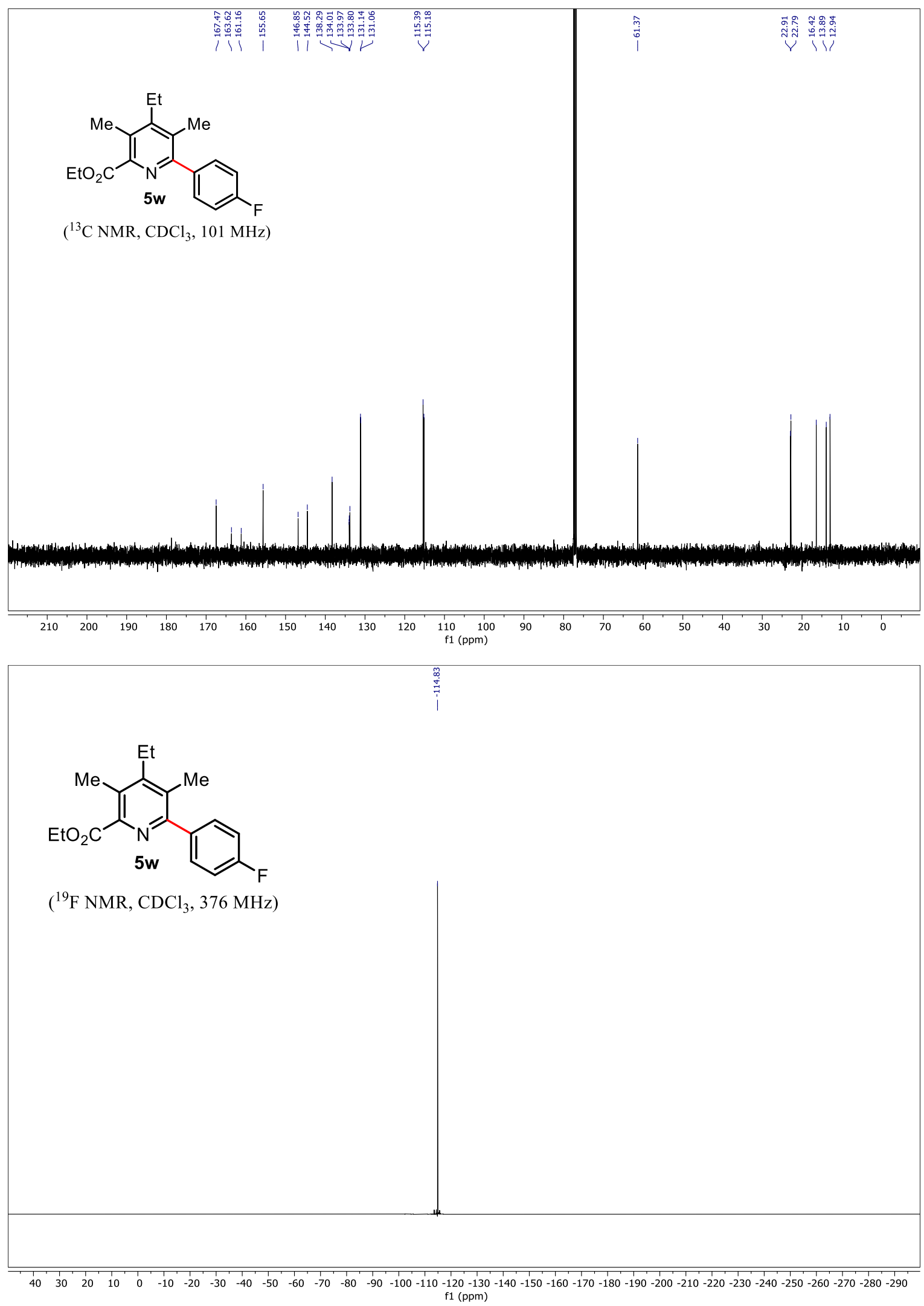
1D NOE NMR spectrum of $\mathbf{5 w}, 500 \mathrm{MHz}, \mathrm{CDCl}_{3}$
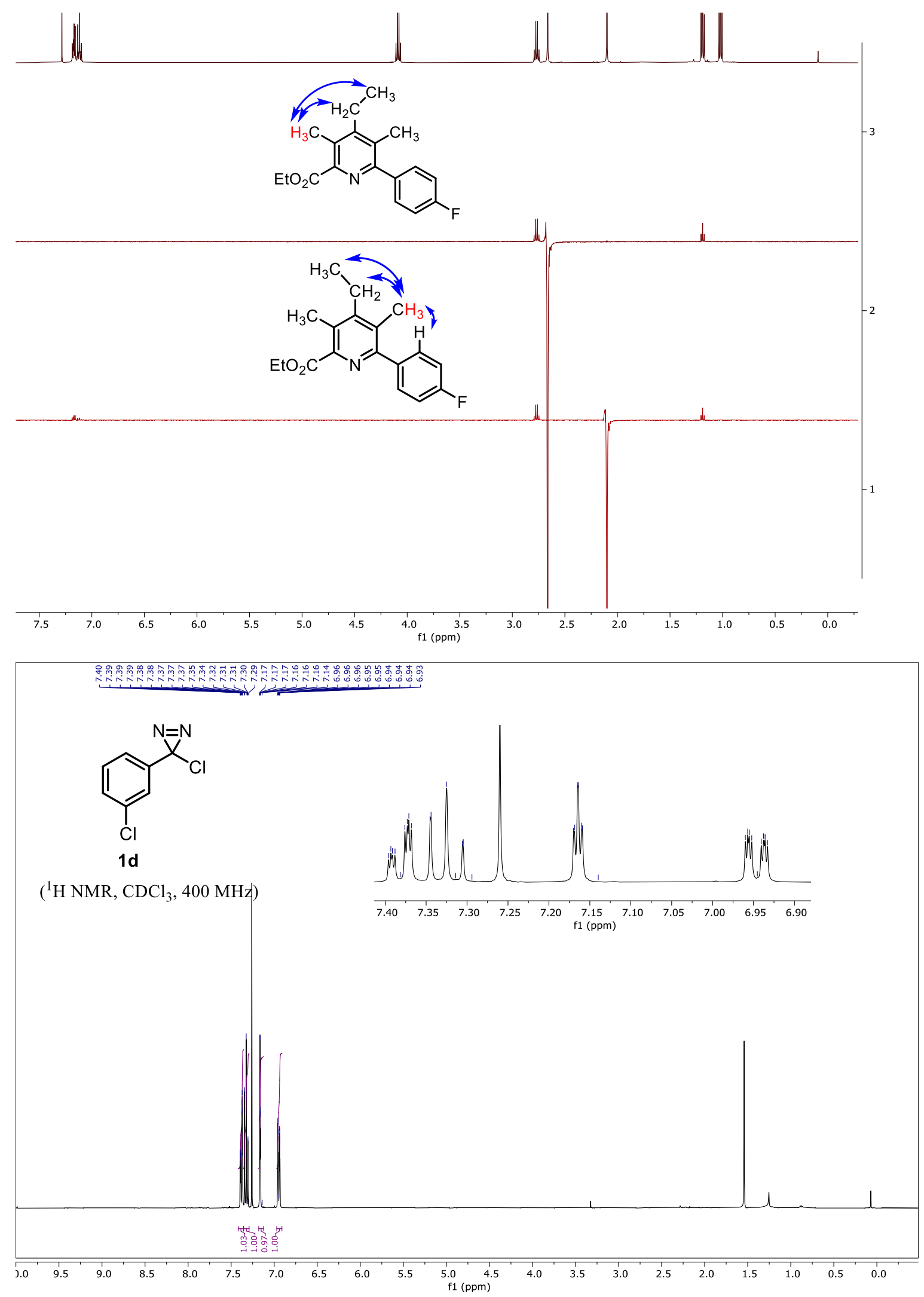

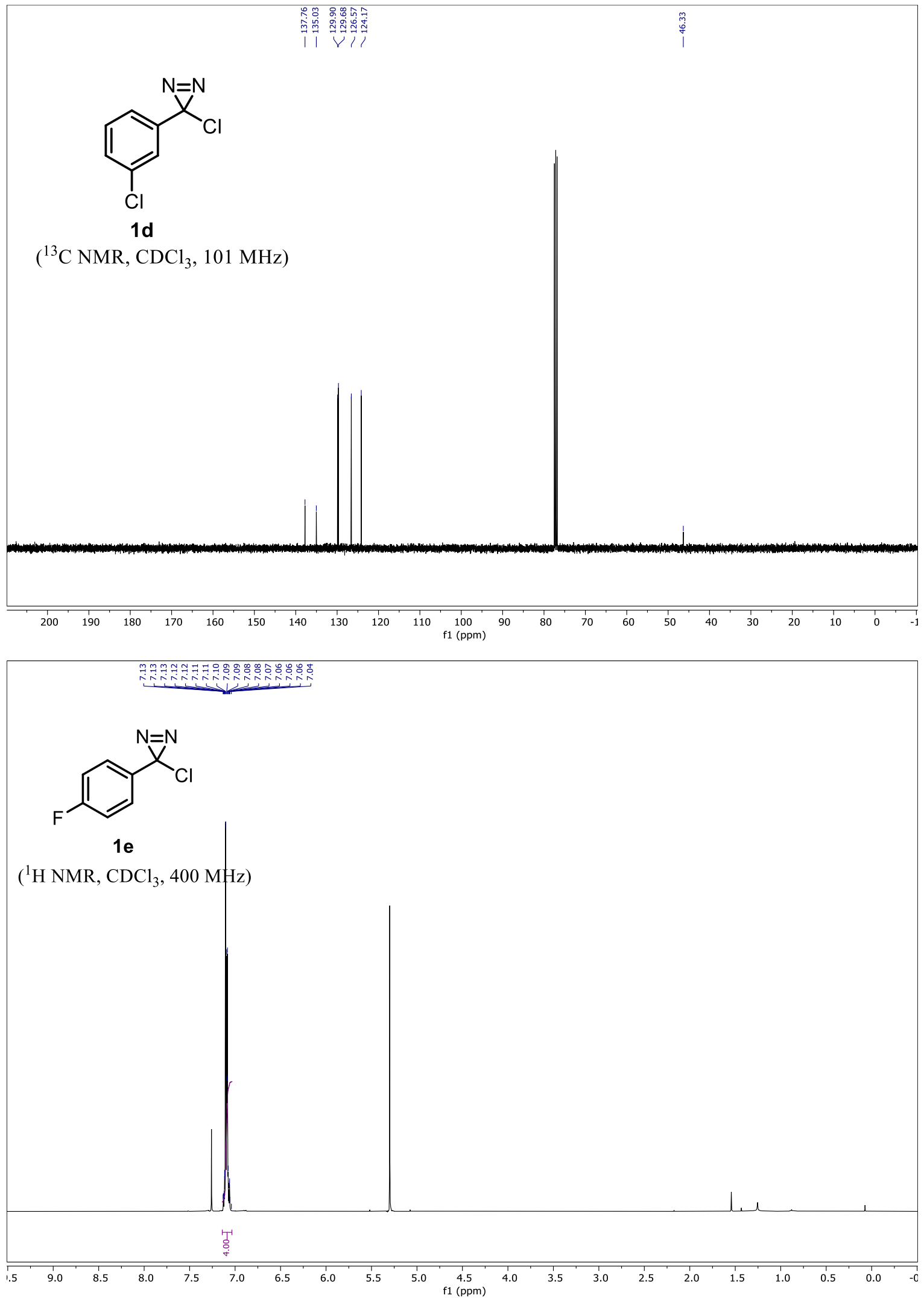

S1-113 

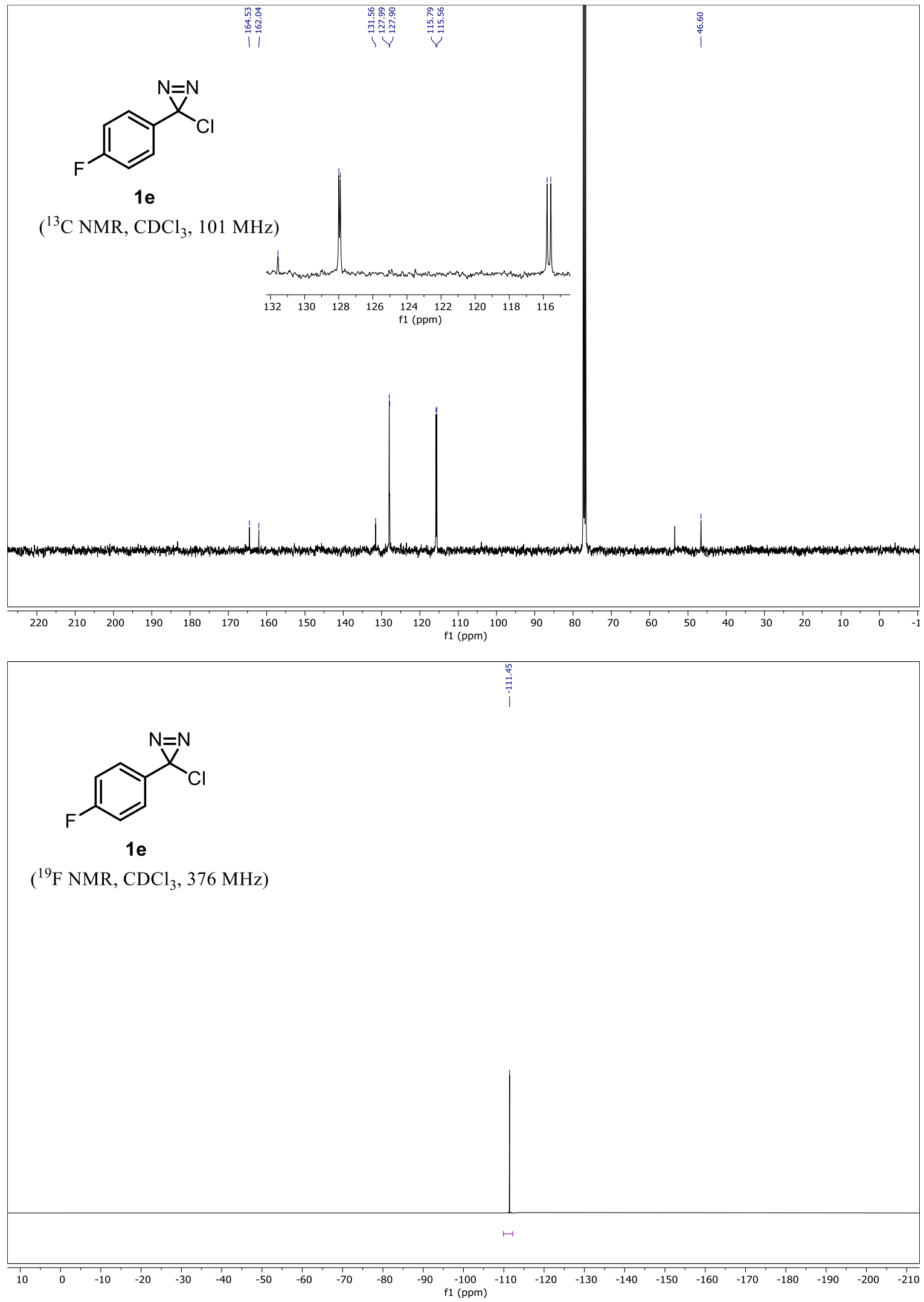

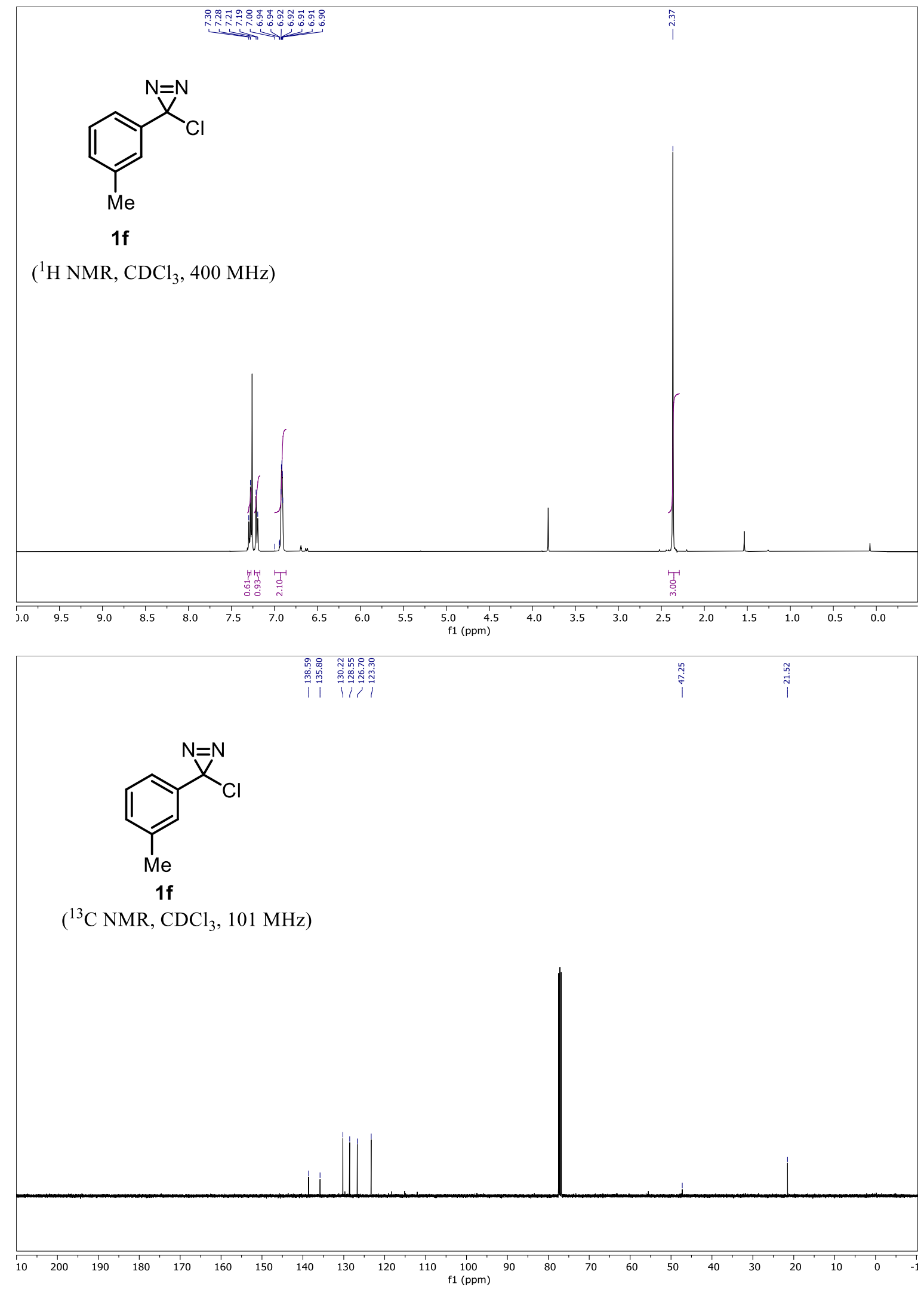

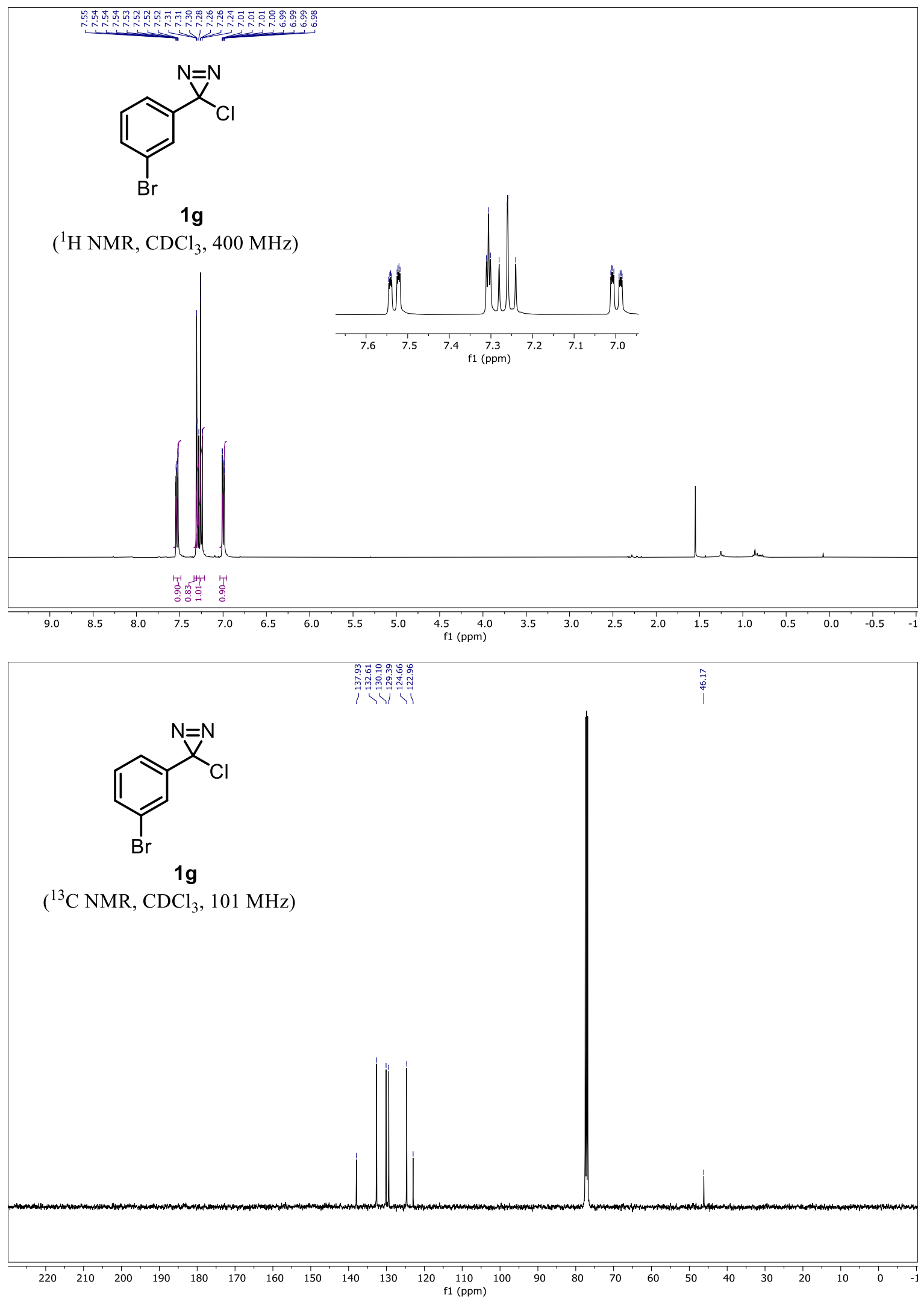

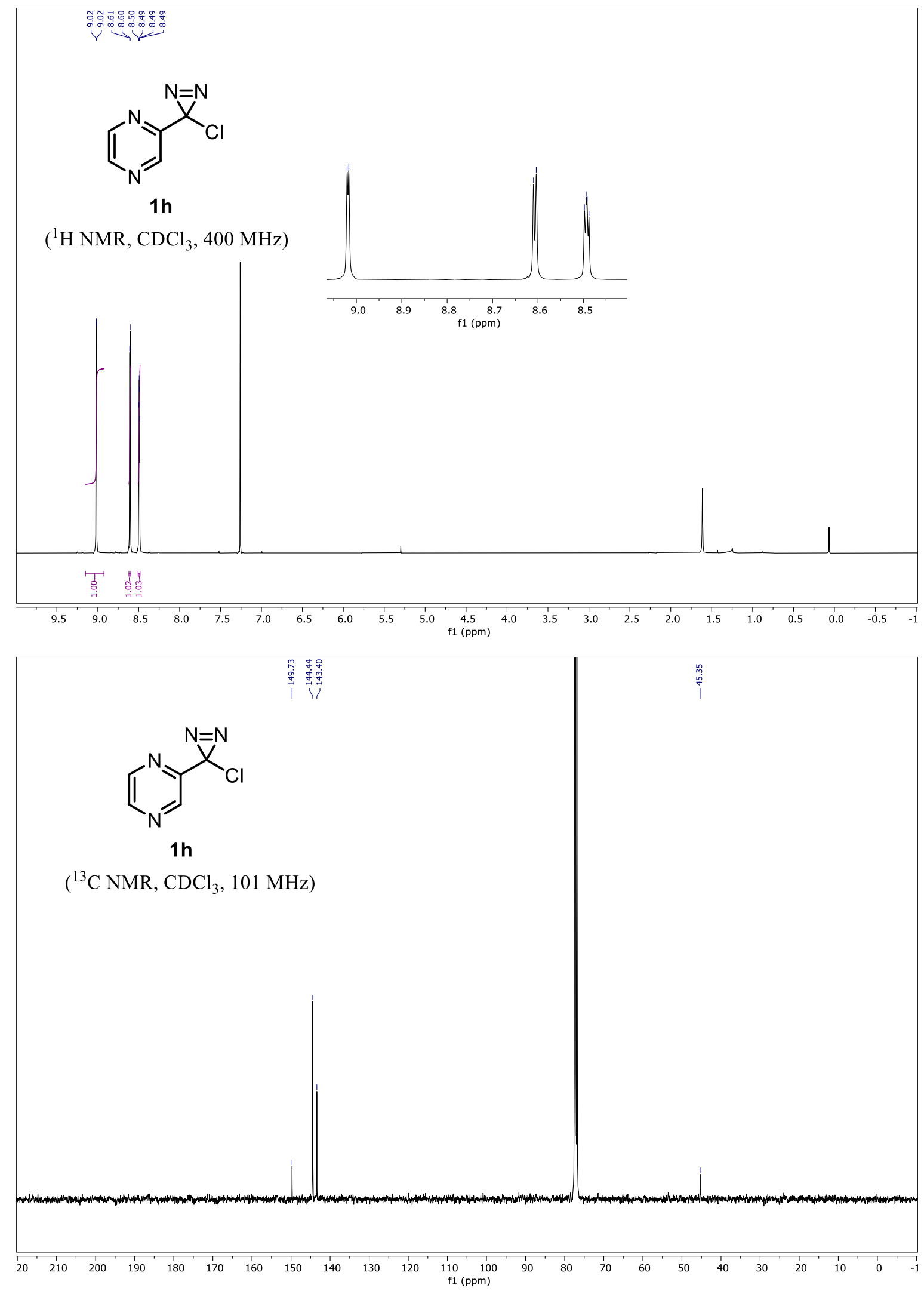

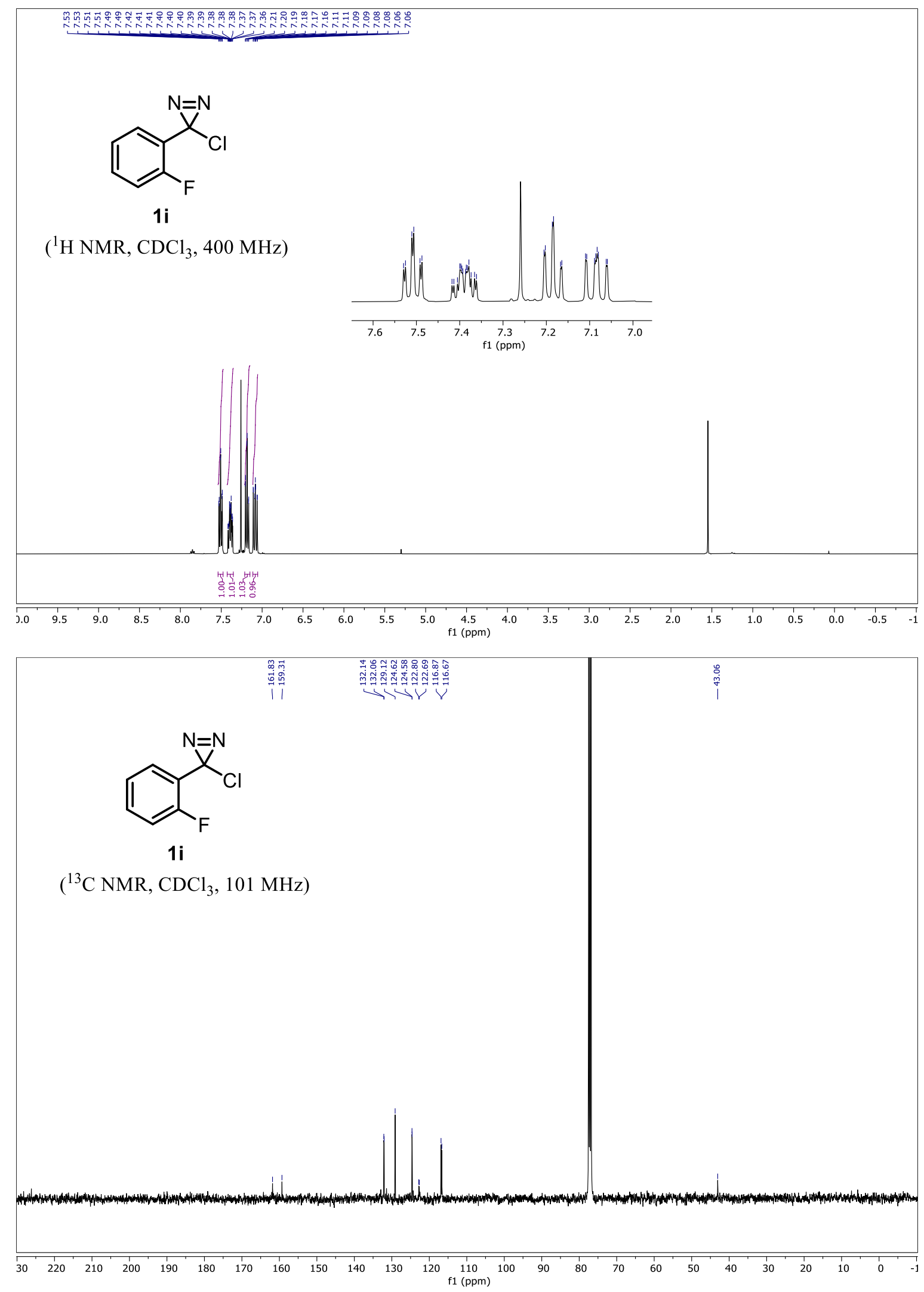

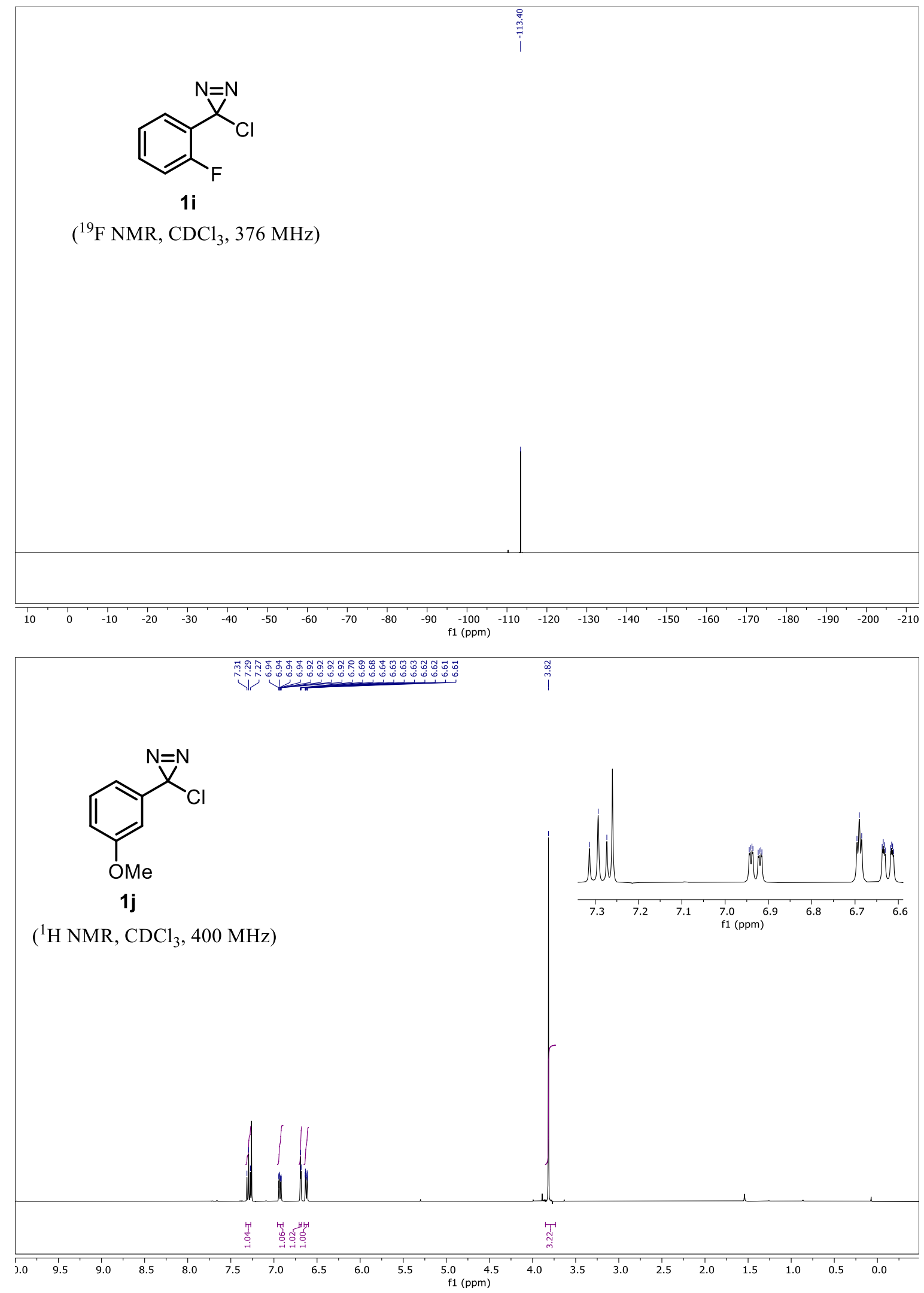

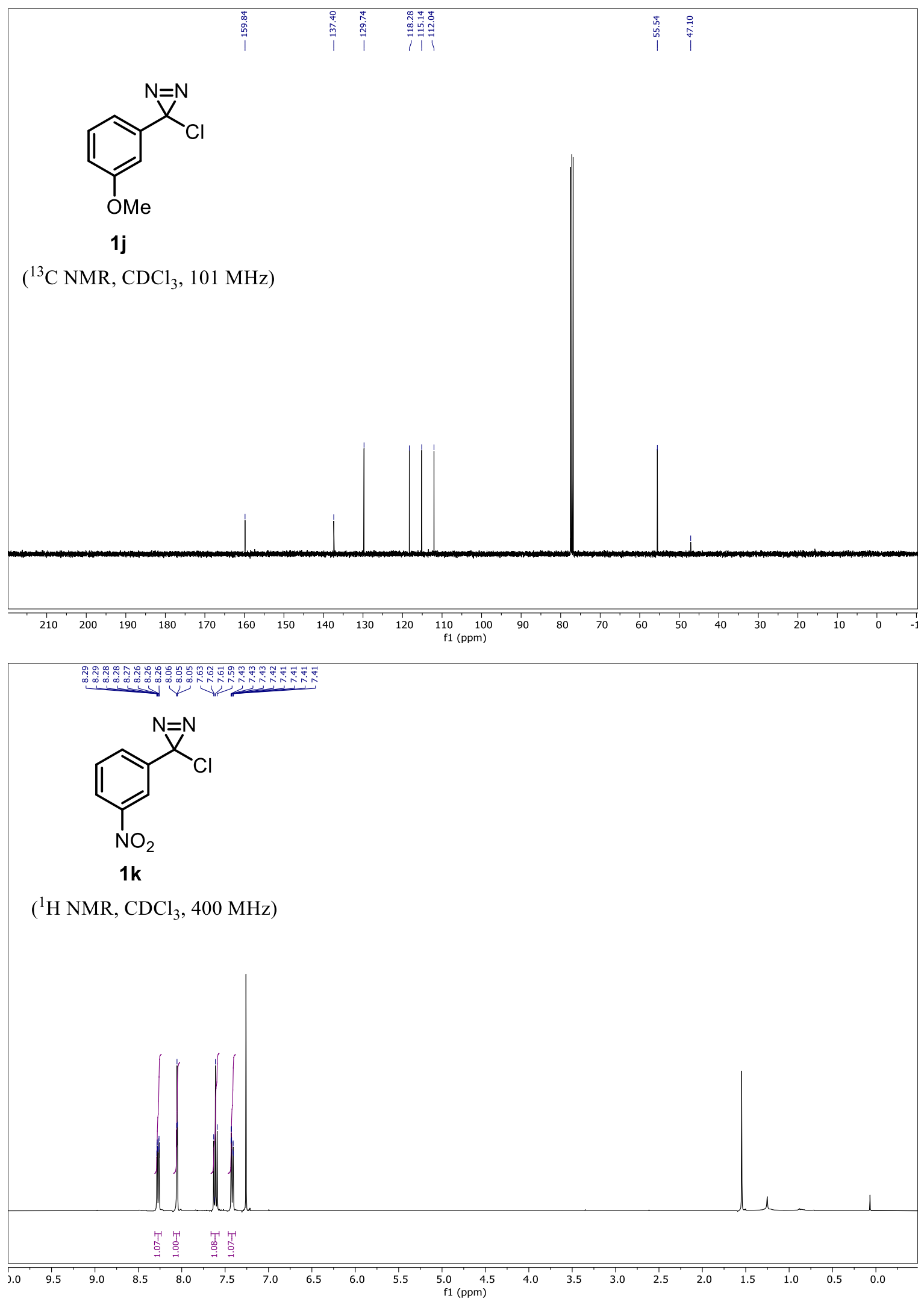

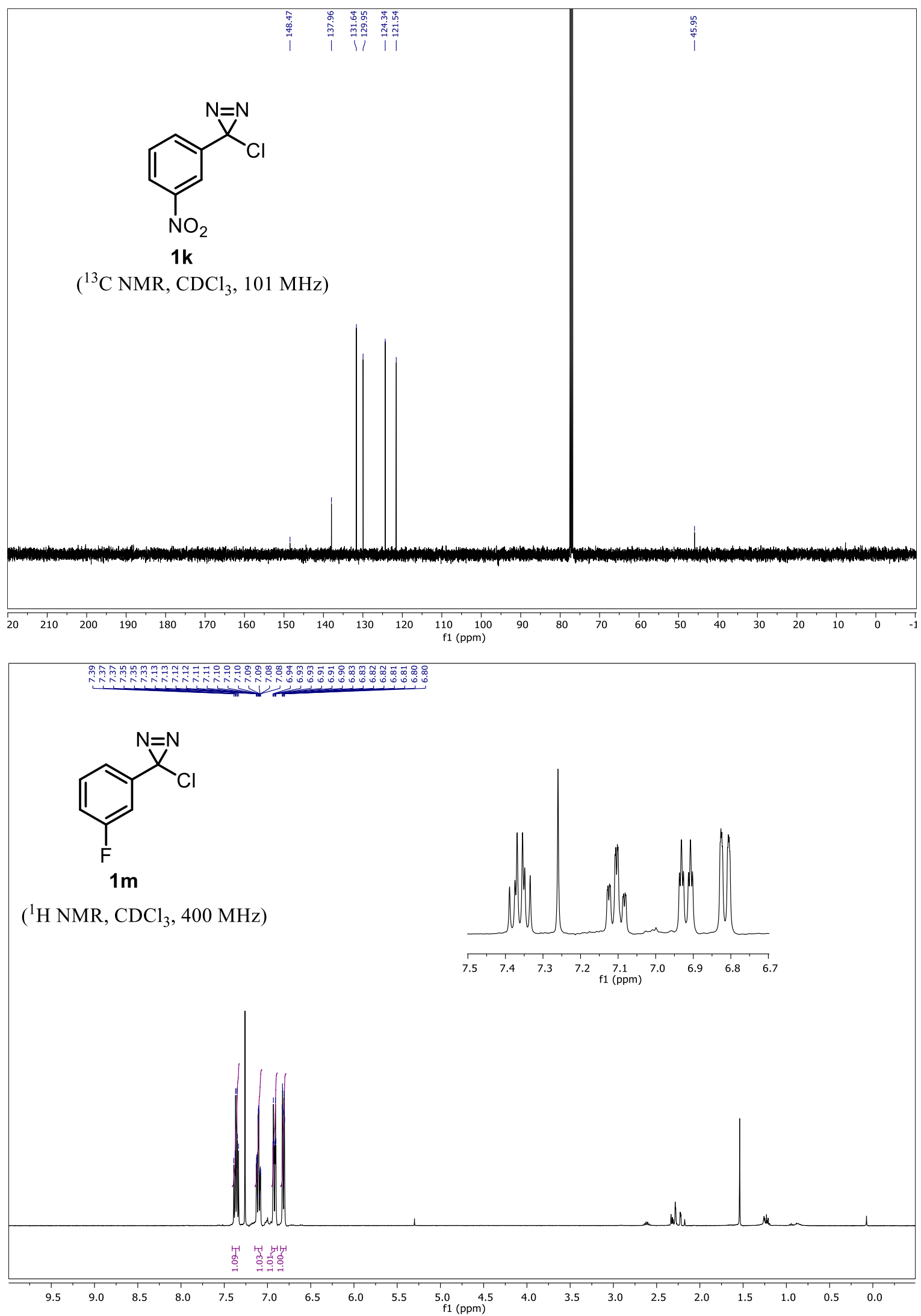

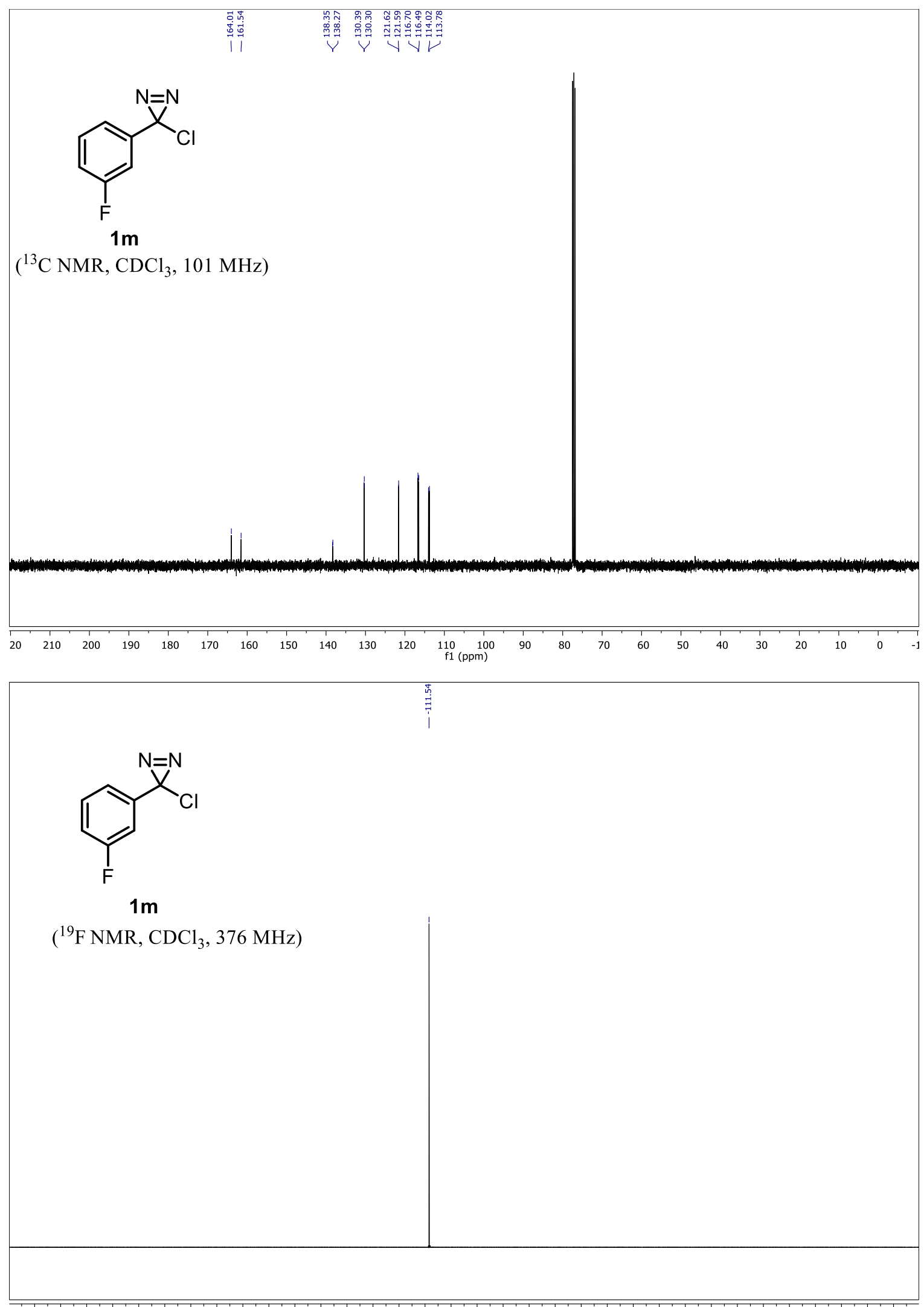

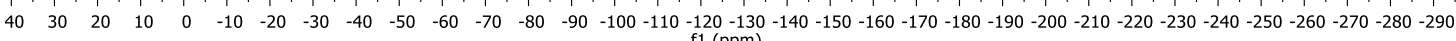



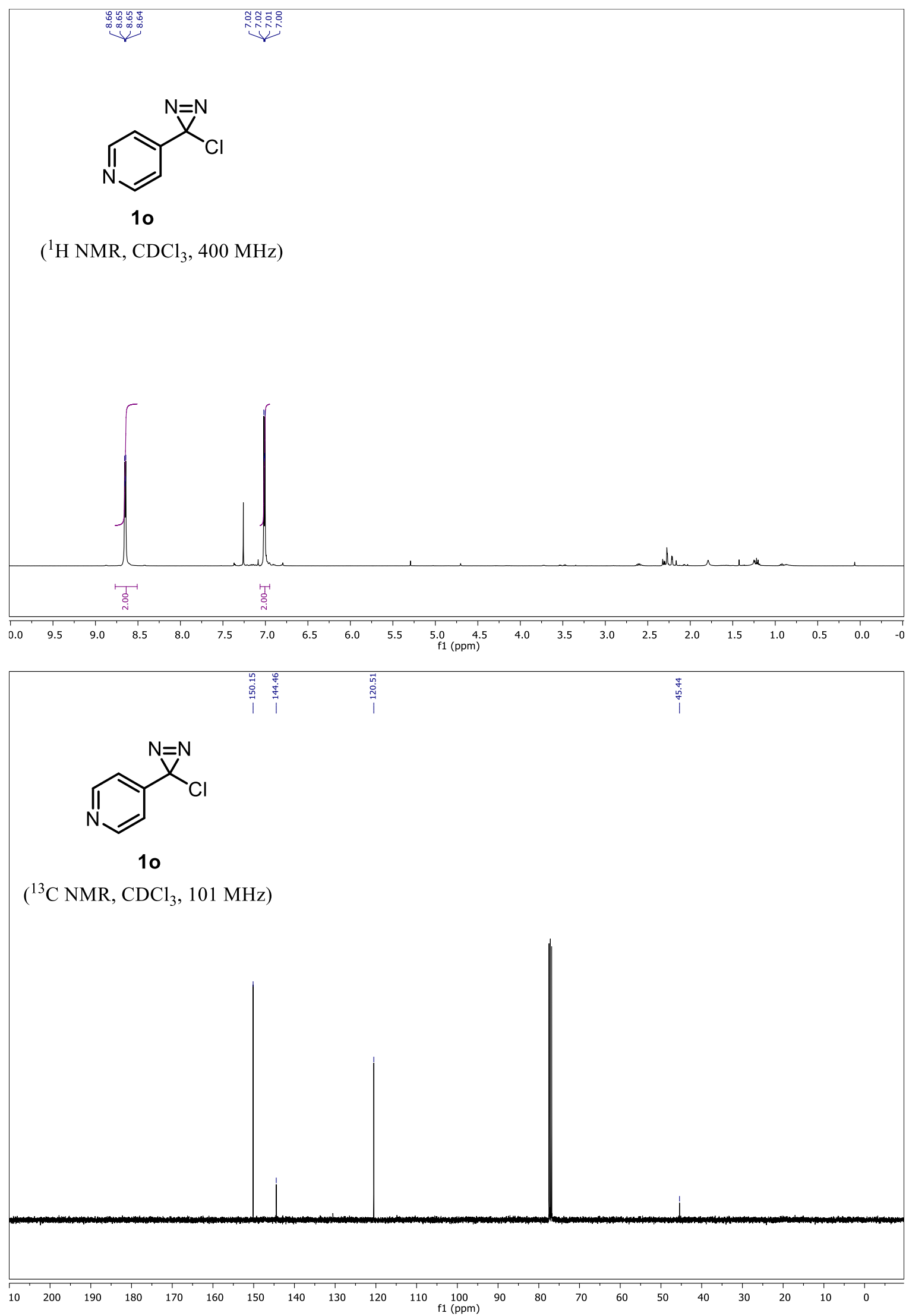

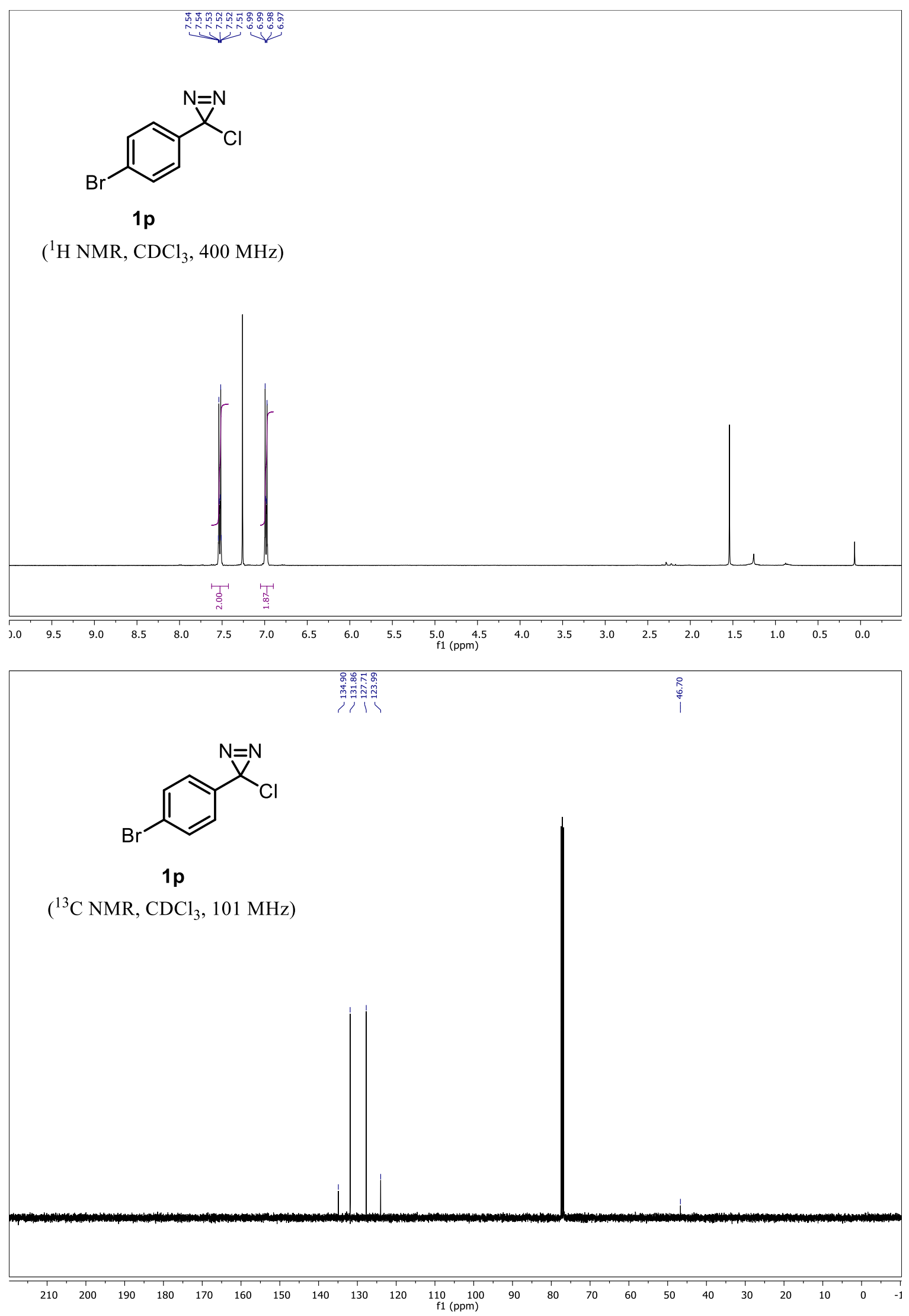

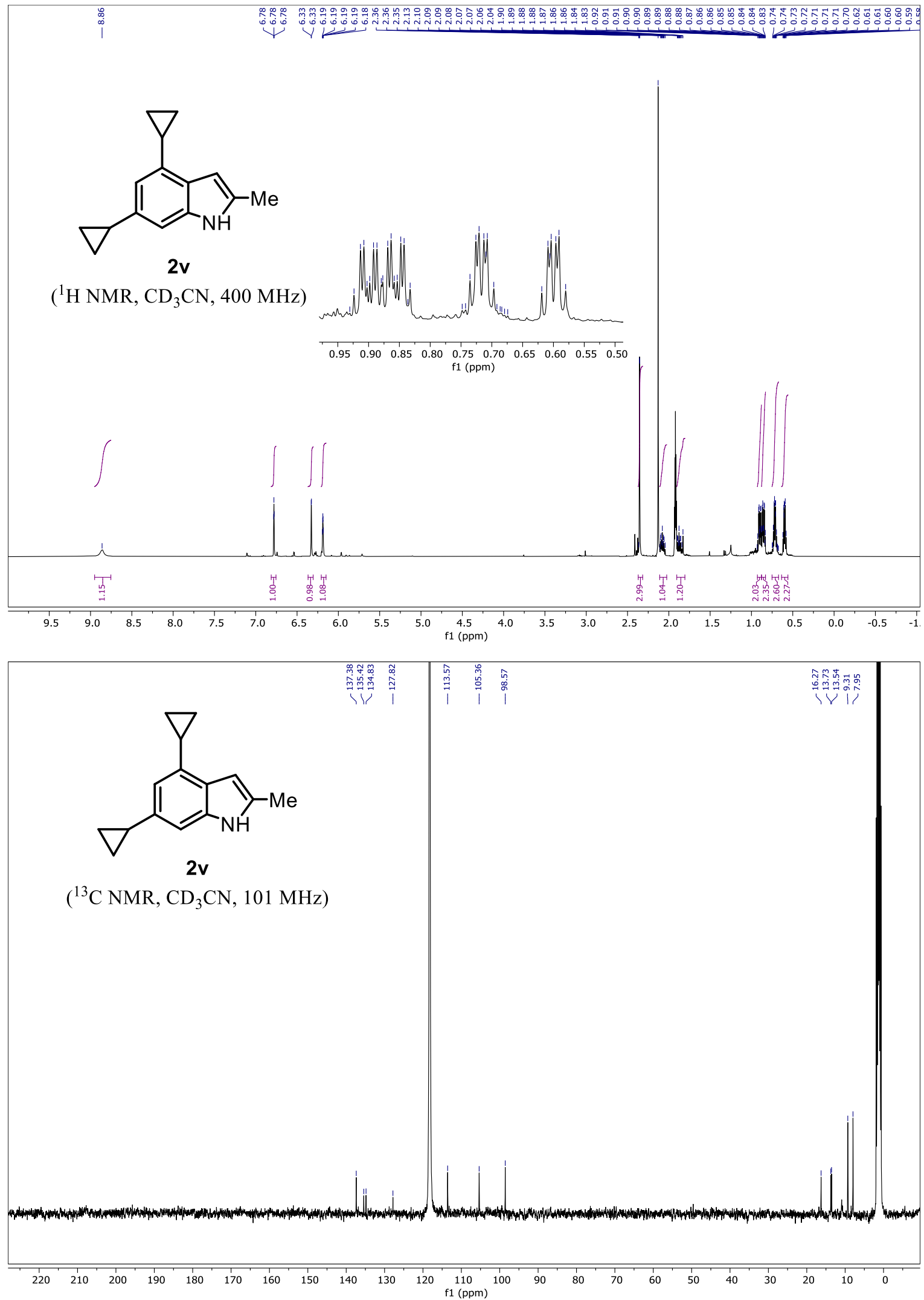

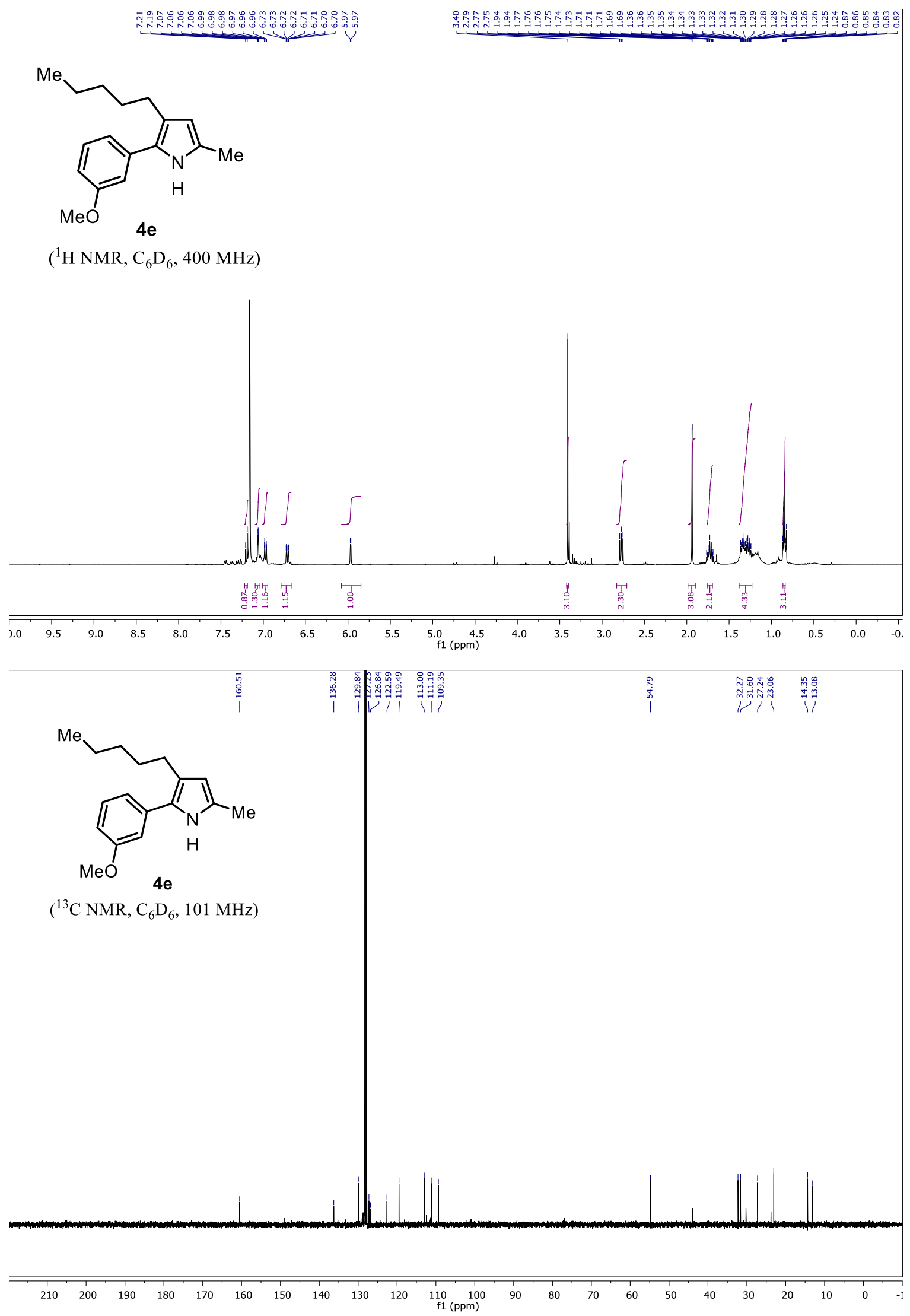

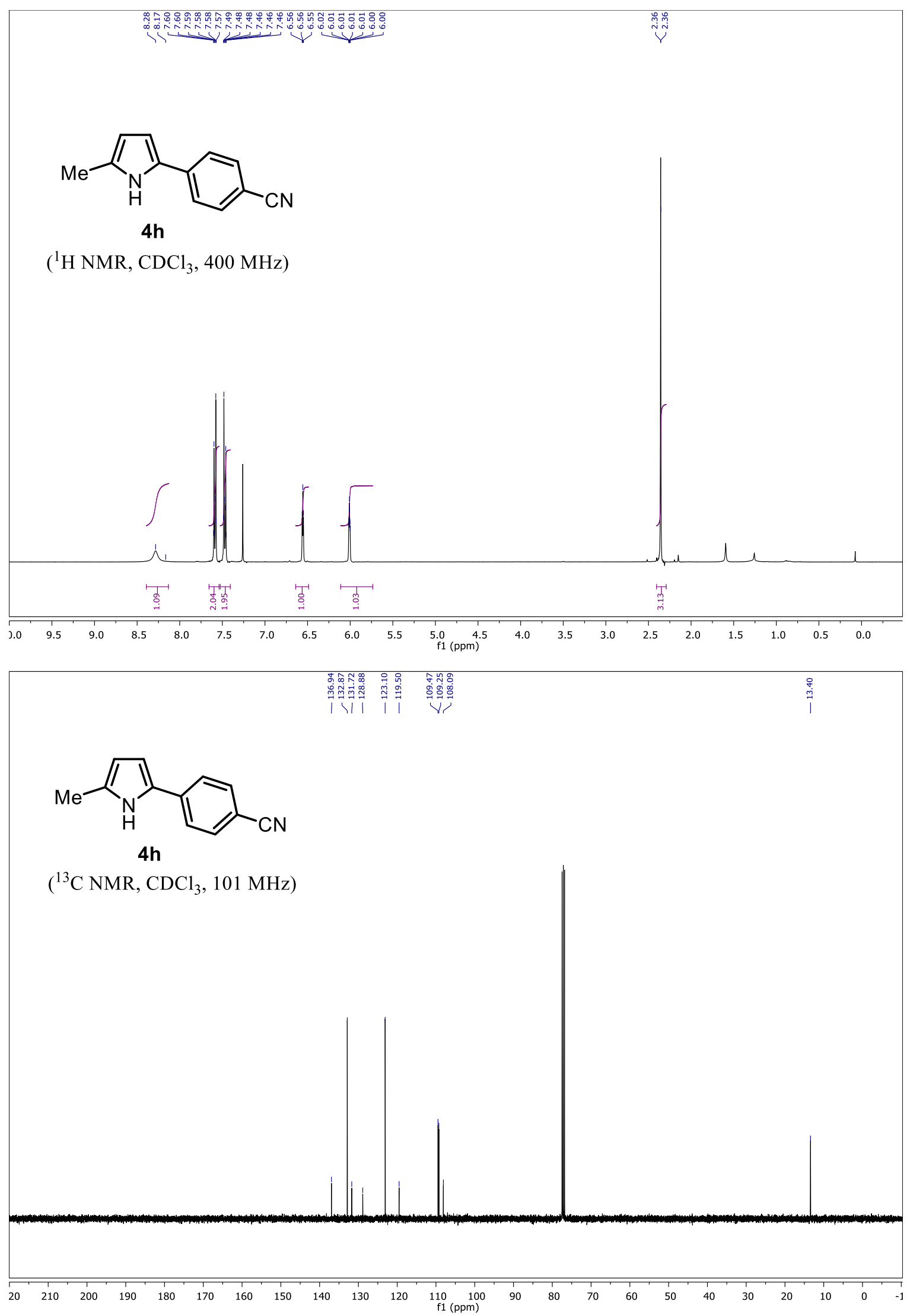

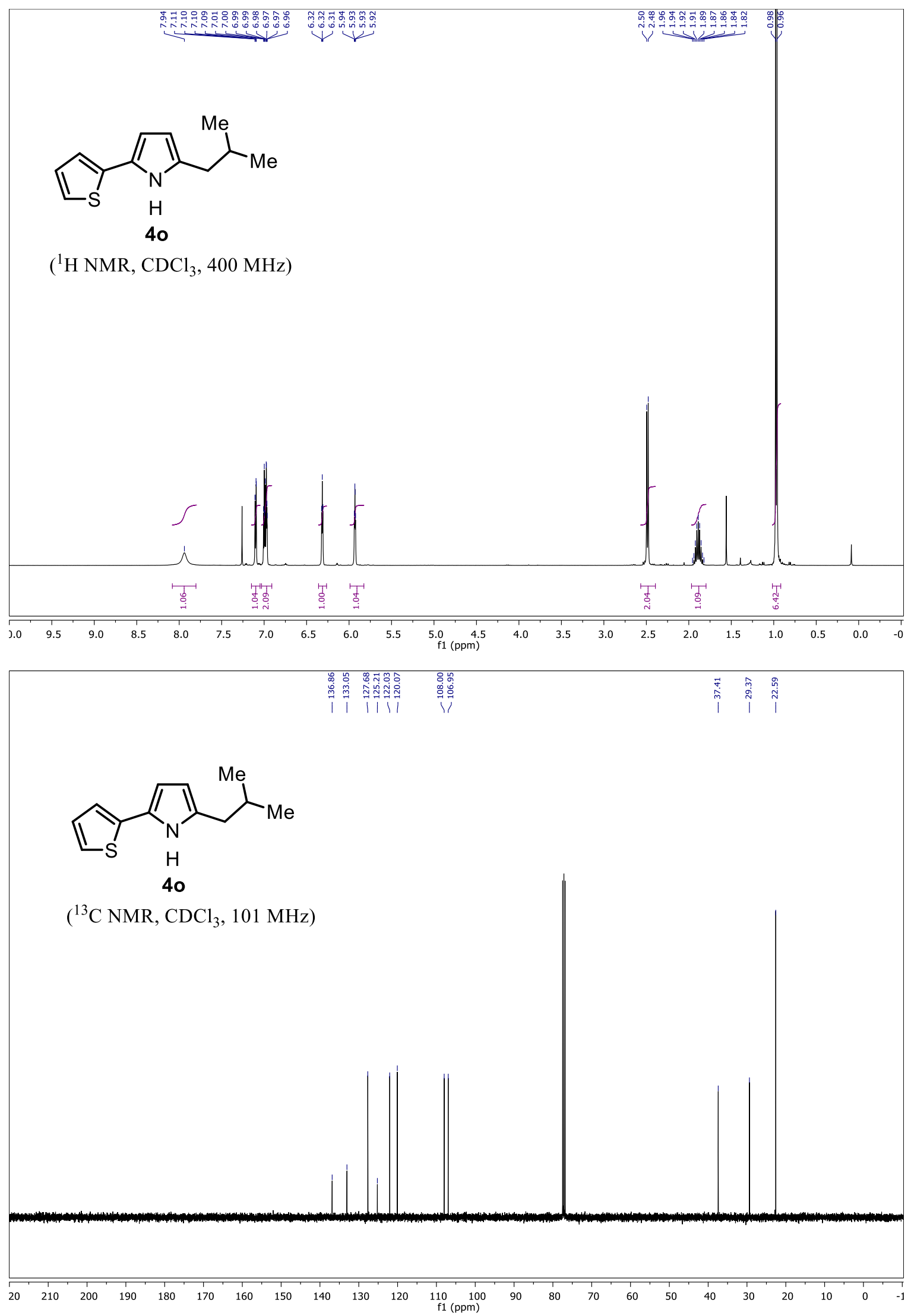

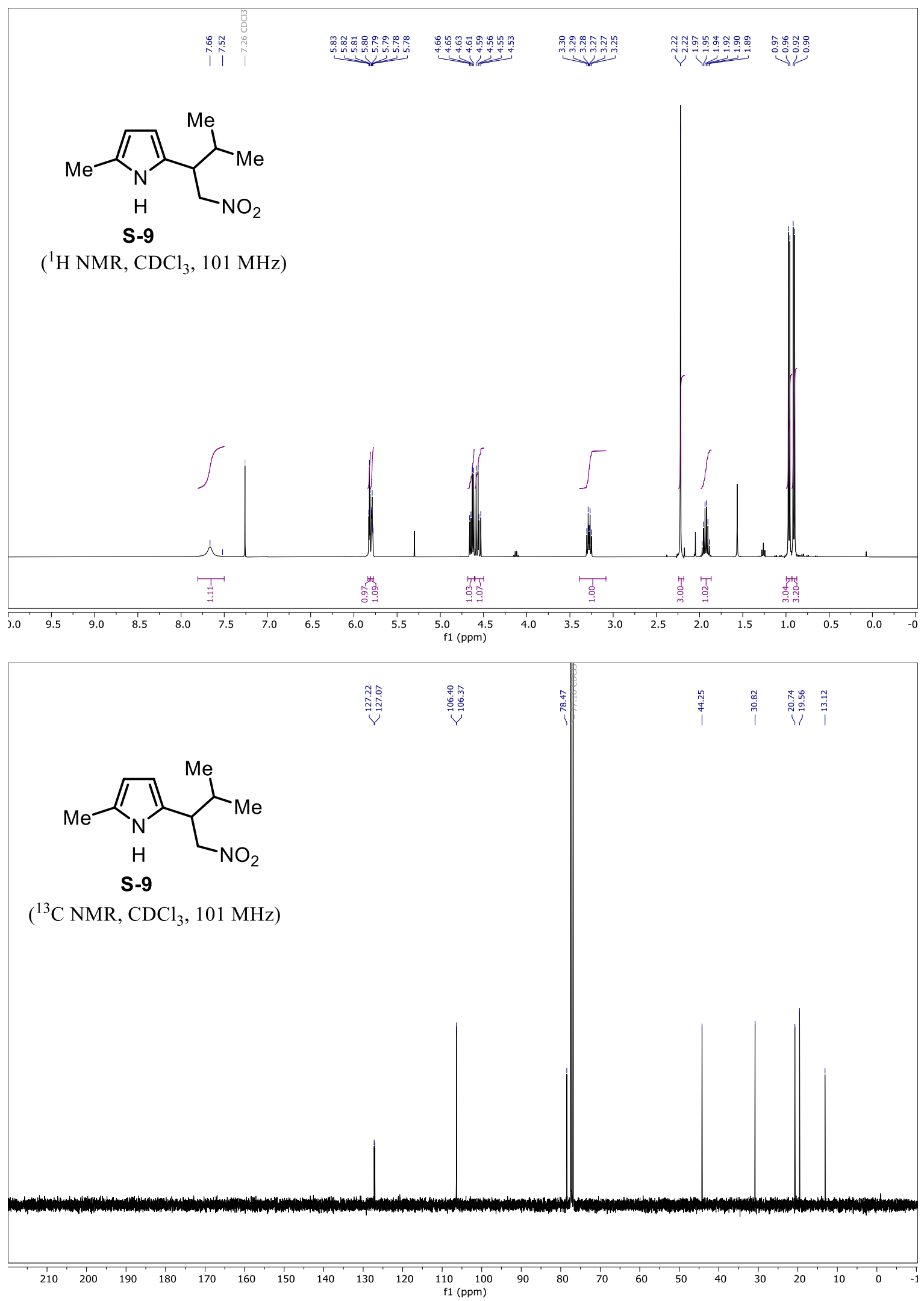

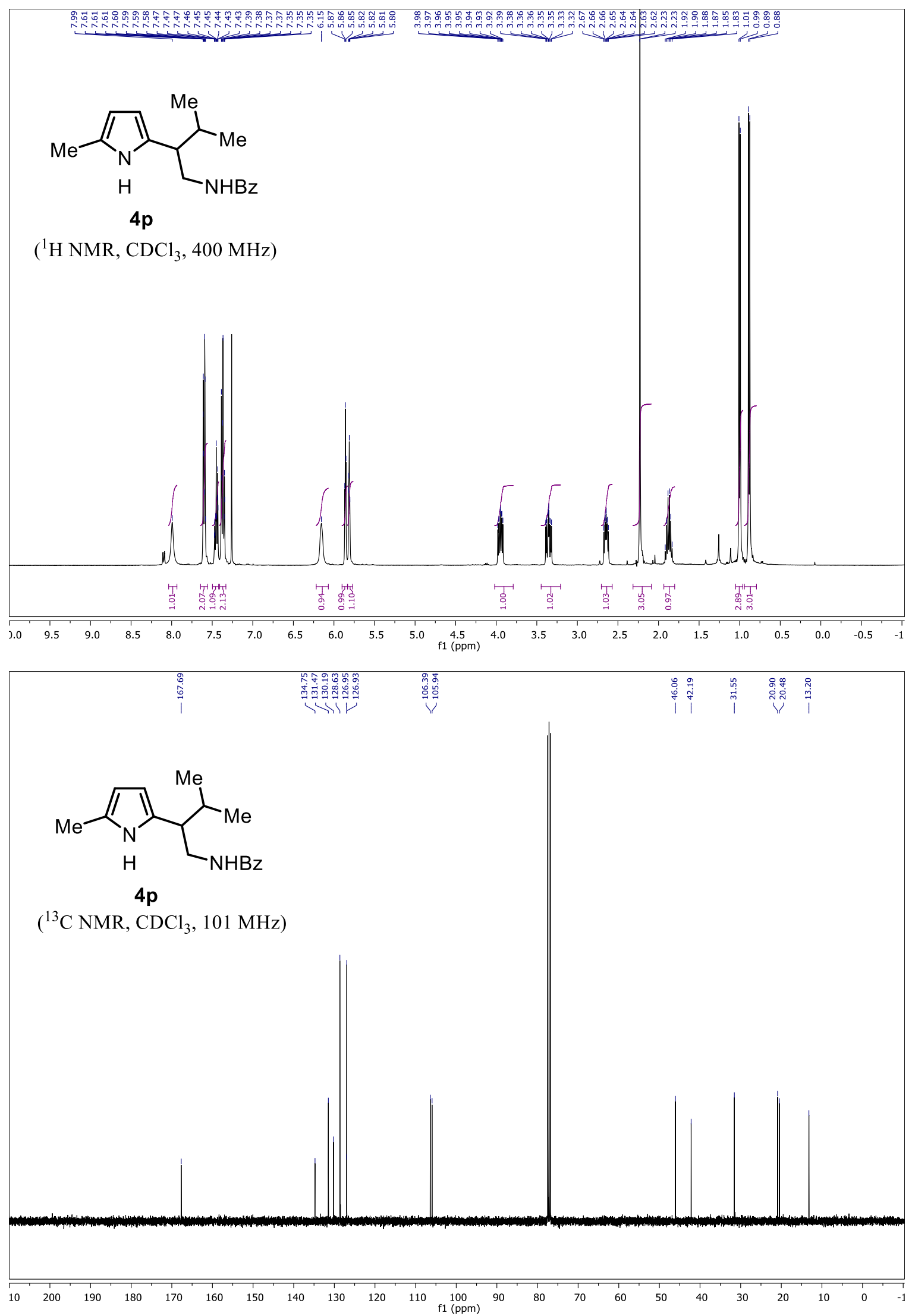

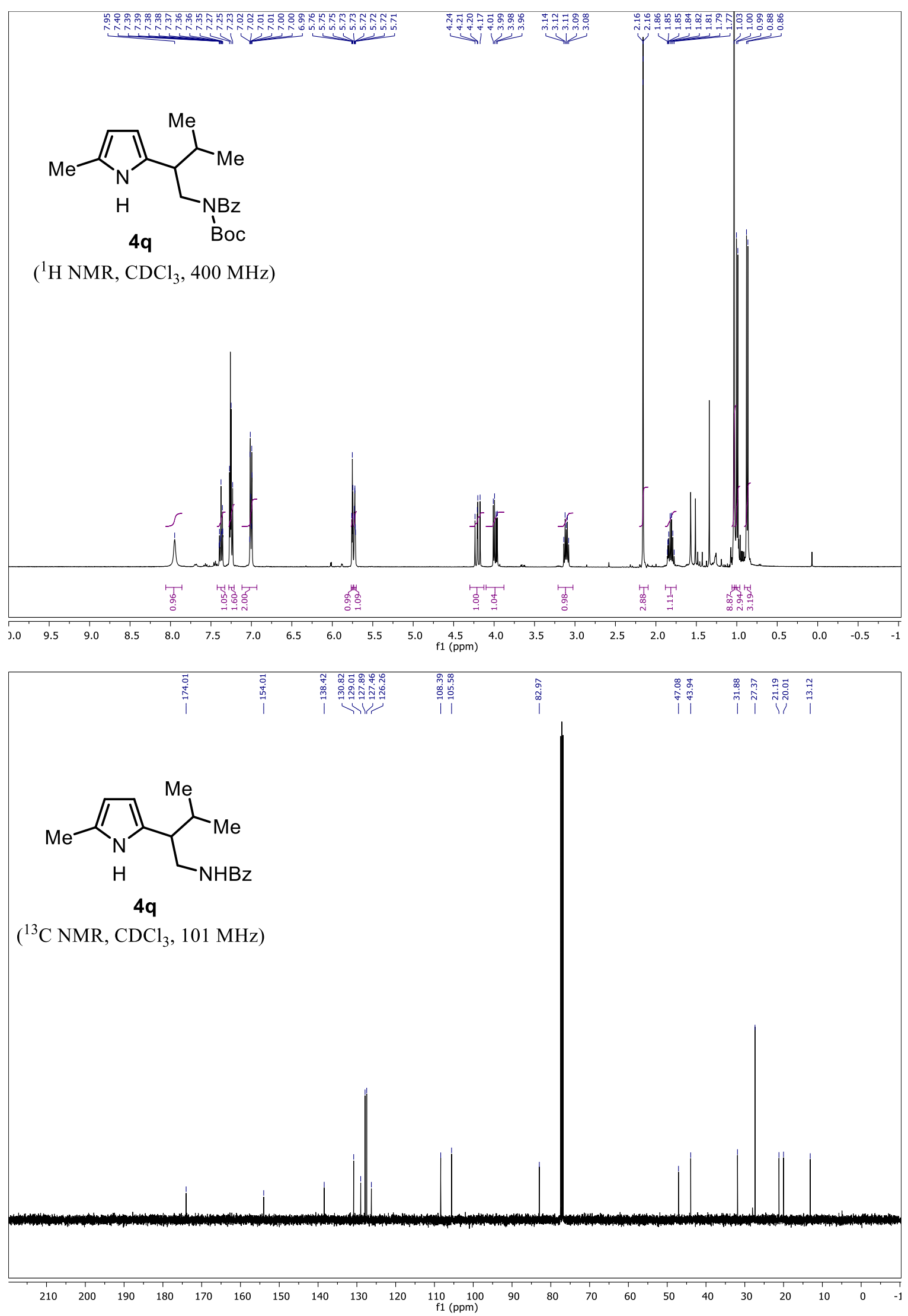

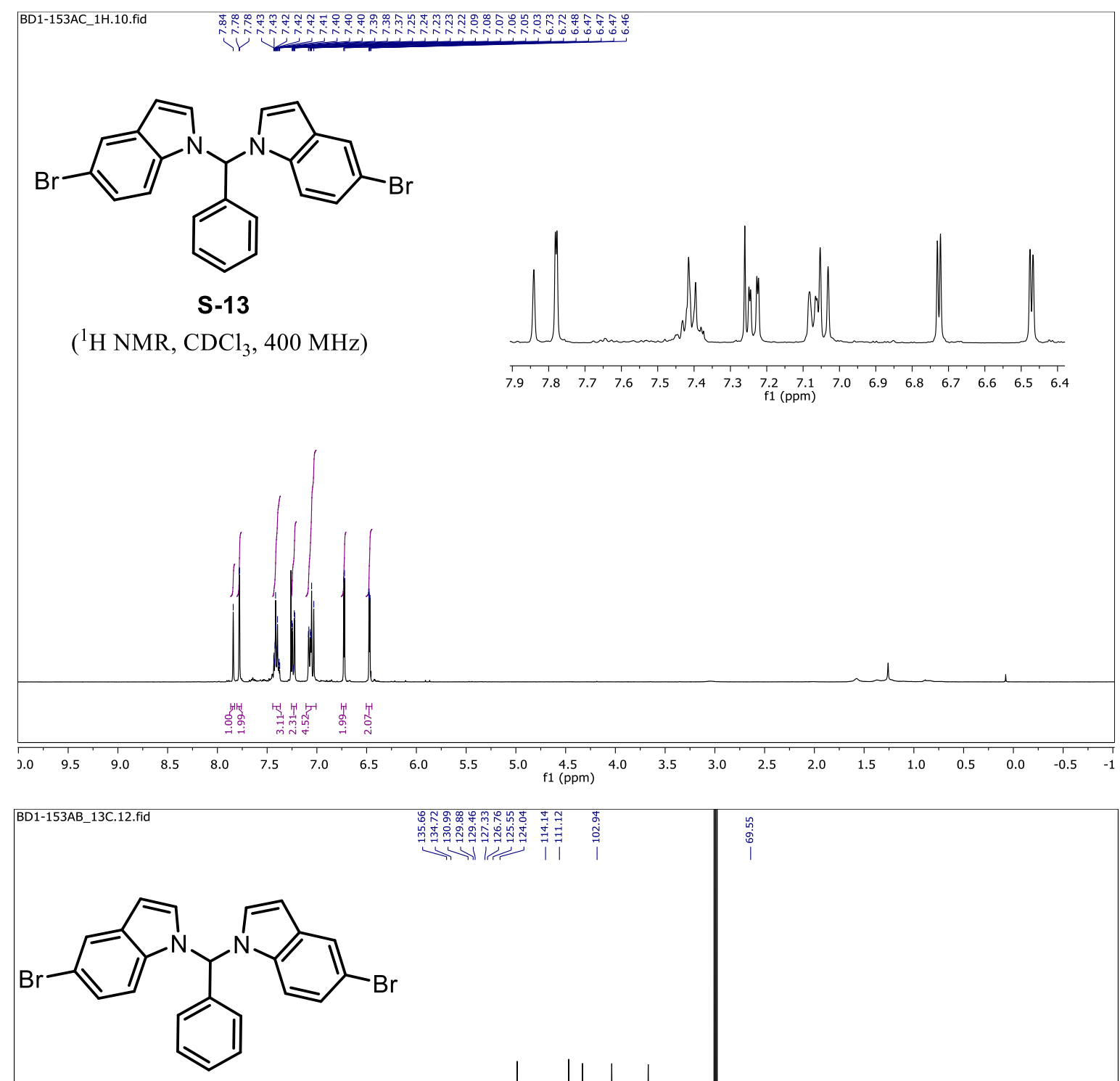

S-13

$\left({ }^{13} \mathrm{C}\right.$ NMR, $\left.\mathrm{CDCl}_{3}, 101 \mathrm{MHz}\right)$

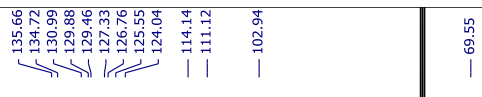

量

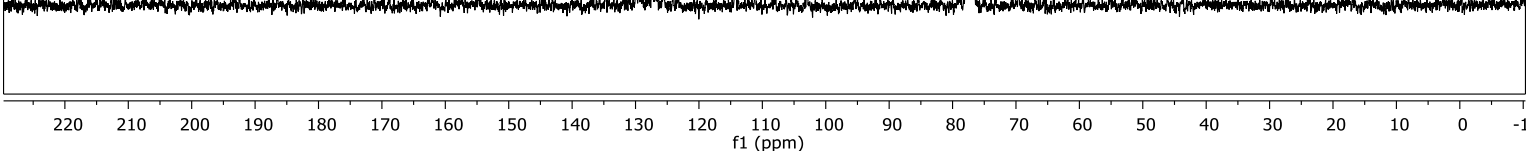

